\title{
The Analysis of Samoan Schools \\ Dropout Rates
}

\author{
A Thesis submitted in fulfillment \\ of the requirements for the \\ Degree of \\ Master of Science in Statistics \\ by
}

Lealaolesau Fitu

School of Mathematics, Statistics and Operations Research

Victoria University of Wellington

P.O. Box 600

Wellington

New Zealand

Victoria University of Wellington 


\section{Abstract}

This thesis investigates the dropout problem in Samoan schools particularly at primary and secondary levels from 1995 to 2007. It aims at identifying and comparing the dropout rate by region (or geographical locations), school level (primary, primary/secondary and secondary) and school status (Government, Mission and Private). Moreover, it also investigates whether the student-teacher ratio, school size, the gender and ethnicity of the teacher, the qualification of the teacher, the school building and school facility variables are associated with the dropout of students. The investigation is carried out through analysis of census data gathered annually by the Samoa Ministry of Education, Sports and Culture (MESC) through census forms from all the primary and secondary schools in the country. Given our response variable is a dichotomous one, the logit regression models to model the effect of both the categorical and continuous explanatory variables on the dropout was adopted. Moreover, since the dropout rates across different year levels (eg, Year 2, Year 3, and Year 13) are correlated within each school we then use the Generalised Estimating Equations (GEE) approach.

The results show that higher dropout rates are found in the rural areas (Rest of Upolu combined with Savaii region) as compared to urban area (Apia urban region). In addition, students are more likely to leave secondary schools earlier than primary or primary/secondary schools. Apparently, the majority of these dropouts are those who have attended Government schools. Surprisingly, students are less likely to get affected with the dropout in larger schools and those schools with higher student-teacher ratios. The gender of the school teacher has nothing to do with the dropout however; having more Samoan and highly qualified teachers in a school will significantly reduce the dropout rate. Nonetheless, a couple of school building variables are significantly associated with the dropout in the positive direction, while another couple inversely relate with the dropout. Schools with more trucks as means of transportation for students, and more radios for school programs and students' activities are less likely to get affected with the dropouts. 


\section{Acknowledgments}

First and foremost I would like to thank our Heavenly Father for granting me with the strength and wisdom to complete this thesis.

I would like to acknowledge and thank my supervisor, Dr Ivy Liu for her professional guidance and support which enabled me to complete this thesis. It has been an honour working with you.

I would like to acknowledge the Head of School of Mathematics, Statistics and Operations Research, Associate Professor Megan Clark for her support and words of encouragement. I would also like to thank the staff in the Statistics and Operations Research Department, particulary Dr Richard Arnold and Dr John Haywood for sharing your knowledge with me. Thank you also to Dr Ray Brownrigg, our Statistical Computing Engineer, who has continually provided the technical support.

Special thanks goes to Professor Neil Quigley, the Deputy Vice Chancellor in Research, and Associate Professor Hon. Luamanuvao Winnie Laban, for giving me the opportunity to complete my thesis. Fa'afetai tele lava.

I would also like to acknowledge the Victoria University of Wellington, NZAID and the Government of Samoa for granting me the financial support in completing this project.

I would also like to acknowledge the support of the current Chief Executive Officer, Afioga Galumalemana Nuufou Petaia and the staff of the Ministry of Education, Sports and Culture, particularly the Policy, Planning and Research Division for your continuous support and assistance.

I would like to acknowledge the support of the Vice Chancellor, Afioga Professor, Lau Asofou So'o and the staff of the National University of Samoa, especially the Dean, Afioga Associate Professor, Muagututia Ioana Chan Mow, Professor Karoline Afamasaga Fuatai and the staff in the Faculty of Science. Thanks very much for words of encouragement and advice.

I would also like to thank my friends and colleagues that we worked together and shared an office with while finishing this thesis. It's been fun working with you guys.

Last but not least, a very special thank you to my parents and families for your support. A special acknowledgement and thank you to my wife Maimoana Petaia-Fitu and our two children, Helena-Marie and Aidan Samuelu for your undying support and love, which have enabled me to complete this thesis. 


\section{Contents}

1 Introduction $\quad 15$

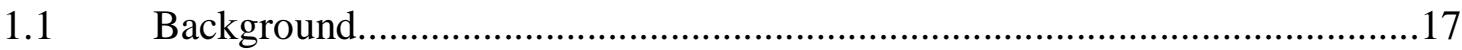

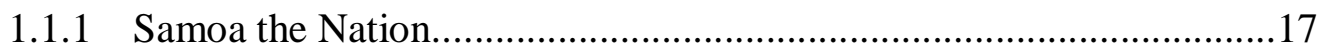

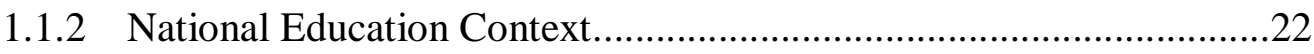

1.2 Ministry of Education, Sports and Culture Survey.........................................29

$2 \quad$ Literature Review $\quad 34$

2.1 The Global Definitions of Dropout or Dropout Rate.......................................35

2.2 Factors associated with Dropout and/or Dropout Rate.....................................39

2.3 Geographical location and Dropout or Dropout Rate......................................46

2.4 School Level and Dropout or Dropout Rate........................................................4

2.5 School Status and Dropout or Dropout Rate.................................................49

2.6 Student-Teacher Ratio and School Size Impact on Dropout or Dropout Rate.50

2.7 School Buildings and School Facilities Impact on Dropout or Dropout Rate.52

$2.8 \quad$ Post-Dropout Drama and Dropout Prevention...................................................53

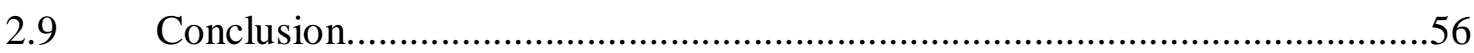

$3 \quad$ The Dropout Rate Overview $\quad 58$

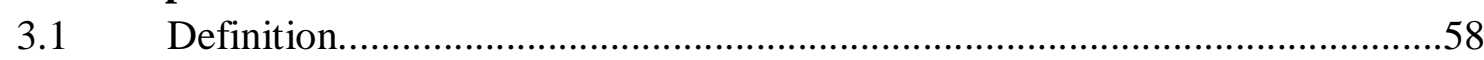

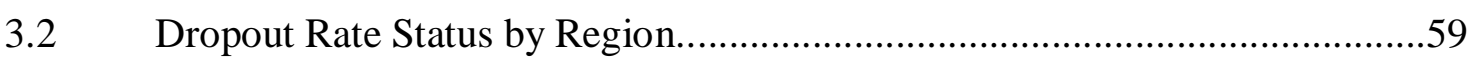

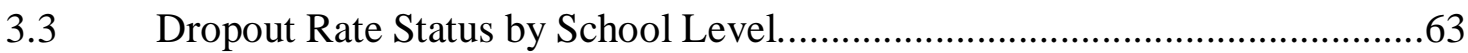

3.4 Dropout Rate Status by School Status.............................................................65

4 Data Analysis and Methodology $\quad \mathbf{7 1}$

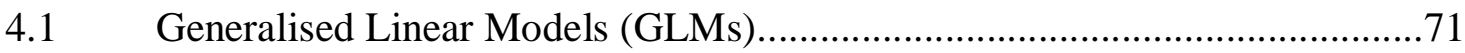

4.1.1 Mean and Variance of a Random Variable.........................................72

4.1.2 Maximum Likelihood Estimates (MLEs)................................................73

4.1.3 Quasi-Likelihood Estimates (QLEs) ..................................................

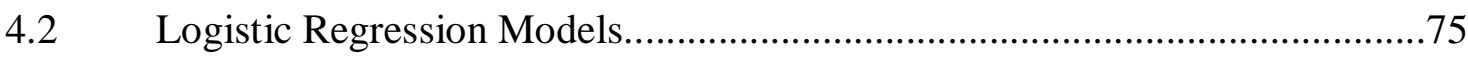

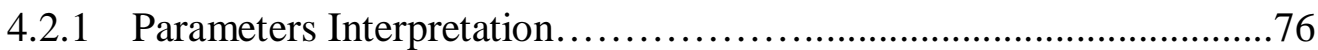

4.2.2 Inferences for Logistic Regression Models........................................77

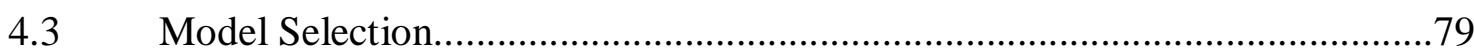

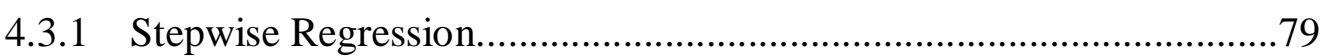

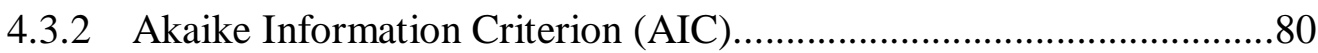

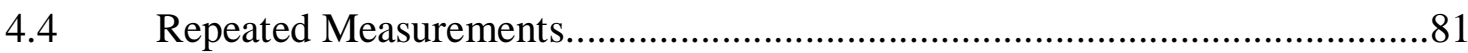

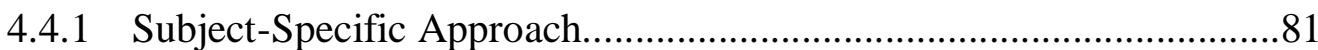

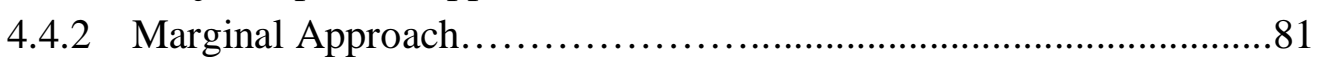


$\begin{array}{lr}\text { Results } & \mathbf{8 4}\end{array}$

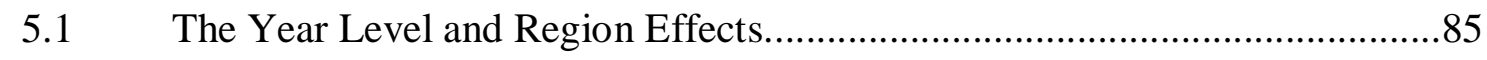

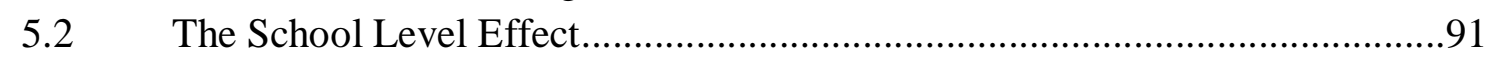

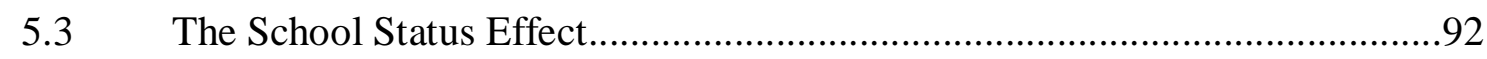

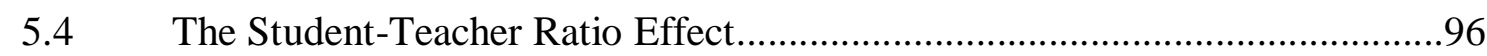

5.5 The School Size (Total Enrolment) Effect.........................................................113

5.6 The Secondary School Teacher Variables Effect (2007)................................114

5.7 The Secondary School Building Effect (2007).............................................116

5.8 The Secondary School Facility Effect (2007).............................................119

6 Discussions and Conclusion $\quad 121$

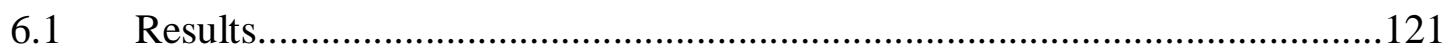

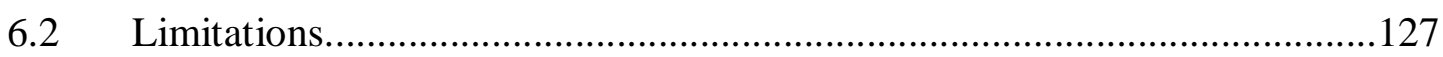

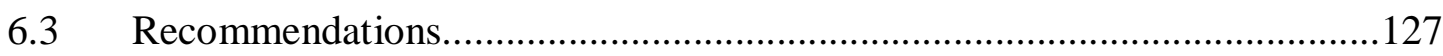

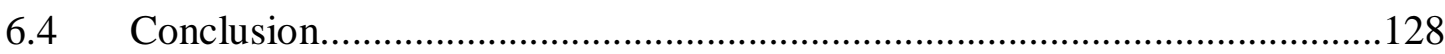

$\begin{array}{ll}\text { Appendices } & 130\end{array}$
A. All Schools' Other Models (1995 - 2006)
130
B. Secondary Schools' Other Models (2007)................................................175

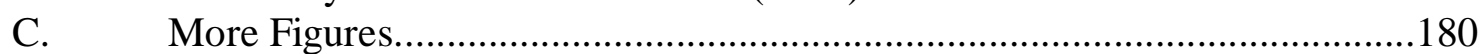

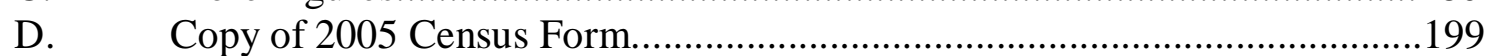

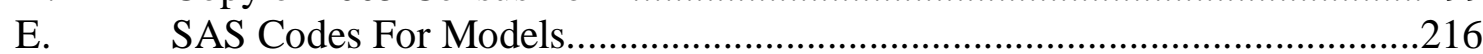
F. The List of the 21 Educational School Districts in Samoa.............................246

References 


\section{ACRONYMS}

$\begin{array}{lll}\text { MESC } & - & \text { Ministry of Education, Sports and Culture } \\ \text { CEO } & - & \text { Chief Executive Officer } \\ \text { ACEOs } & - & \text { Assistant Chief Executive Officers } \\ \text { SRO } & - & \text { School Review Officers } \\ \text { PPRD } & - & \text { Policy, Planning and Research Division } \\ \text { SOD } & - & \text { School Operation Division } \\ \text { CMAD } & - & \text { Curriculum, Materials and Assessment Division } \\ \text { CSD } & - & \text { Coporate Services Division } \\ \text { SD } & - & \text { Sports Division } \\ \text { CD } & - & \text { Culture Division } \\ \text { EFA } & - & \text { Education For All } \\ \text { MDGs } & - & \text { Millenium Development Goals } \\ \text { SQA } & - & \text { Samoa Qualifications Authority } \\ \text { NUS } & - & \text { National University of Samoa } \\ \text { FOE } & - & \text { Faculty of Education } \\ \text { ESPII } & - & \text { Education Sector Project II } \\ \text { UNESCO } & - & \text { United Nations Education, Science and Culture Organisation }\end{array}$




\section{List of Tables}

Table 1.1: Estimates of Land Use in Samoa........................................................................

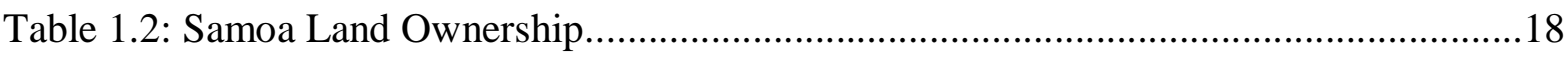

Table 1.3: Total Population of Samoa in 2011 and 2006......................................................19

Table 1.4: Number of Primary and Secondary schools for each Educational Region by School

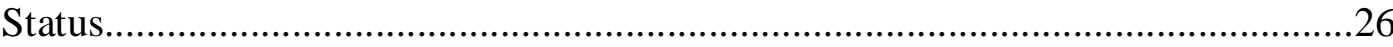

Table 1.5: The Distribution of schools into Control Authority by School Status ...................26

Table 1.6: The Distribution of schools into Regions by School Type.....................................27

Table 1.7: The Distribution of schools into School Status by School Level.............................27

Table 1.8: Primary Schools 2007 enrolment by School Status...............................................28

Table 1.9: Number of Schools in Samoa by School Level in 2007 .......................................28

Table 3.1: School Year Level Median Dropout Rates of all schools by Region......................60

Table 3.2: School Year Level Median Dropout Rates of all schools by School Level...........64

Table 3.3: School Year Level Median Dropout Rates of all schools by School Status...........66

Table 5.1: Estimates and Odds Ratio for Year Level and Region in 1995.............................87

Table 5.2: Chi-Square Statistics of the Model for Year Level and Region in 2000................87

Table 5.3: Estimates and Odds Ratio for Year Level and Region in 2003.............................88

Table 5.4: Estimates and Odds Ratio for Year Level and Region in 2006..............................89

Table 5.5: Estimates and Odds Ratio for Year Level and School Level in 1995 _....................92

Table 5.6: Estimates and Odds Ratio for Year Level and School Status in 1999....................93

Table 5.7: Estimates and Odds Ratio for Year Level and School Status in 2000....................94

Table 5.8: Estimates and Odds Ratio for Year Level and School Status in 2003....................95

Table 5.9: Chi-Square Statistics for the Model with four Co-variates (1995)..........................99

Table 5.10: Final Model for the Student-Teacher ratio effect (1995)......................................99

Table 5.11: Chi-Square Statistics for the Model with four Co-variates (1996).......................99

Table 5.12: Final Model for the Student-Teacher ratio effect (1996)...................................100

Table 5.13: Final Model for the Student-Teacher ratio effect (1997)...................................101 
Table 5.14: Chi-Square Statistics for the Model with four Co-variates (1999). 102

Table 5.15: Final Model for the Student-Teacher ratio effect (1999)_..................................102

Table 5.16: Chi-Square Statistics for the Model with four Co-variates (2000).....................103

Table 5.17: Final Model for the Student-Teacher ratio effect (2000)...................................103

Table 5.18: Chi-Square Statistics for the Model with four Co-variates (2001).....................103

Table 5.19: Final Model for the Student-Teacher ratio effect (2001)...................................104

Table 5.20: Chi-Square Statistics for the Model with four Co-variates (2004).....................107

Table 5.21: Final Model for the Student-Teacher ratio effect (2004)..................................108

Table 5.22: Chi-Square Statistics for the Model with four Co-variates (2005).....................109

Table 5.23: Final Model for the Student-Teacher ratio effect (2005)...................................109

Table 5.24: Chi-Square Statistics for the Model with four Co-variates (2006).....................111

Table 5.25: Final Model for the Student-Teacher ratio effect (2006).................................112

Table 5.26: The Model for the School Size (Total Enrolment) effect (1995)........................114

Table 5.27: The Model for the School Size (Total Enrolment) effect (2006).........................114

Table 5.28: The Chi-Square Statistics of Full Model for 2007 Secondary School Teacher Variables.

Table 5.29: The Simpler Model for 2007 Secondary School Teacher Variables....................116

Table 5.30: The Simpler Model for 2007 Secondary School Building Variables..................117

Table 5.31: The Chi-Square Statistics of Full Model for 2007 Secondary School Building Variables. 118

Table 5.32: The Simpler Model for 2007 Secondary School Facility Variables. 119

Table 5.33: The Chi-Square Statistics of Full Model for 2007 Secondary School Facility Variables.

A. R.1: Estimates and Odds Ratio for Year Level and Region in 1996.................................130

A. R.2: Estimates and Odds Ratio for Year Level and Region in 1997...............................131

A. R.3: Estimates and Odds Ratio for Year Level and Region in 1998_................................132

A. R.4: Estimates and Odds Ratio for Year Level and Region in 1999................................133

A. R.5: Estimates and Odds Ratio for Year Level and Region in 2000.................................134 
A. R.6: Estimates and Odds Ratio for Year Level and Region in 2001...............................135

A. R.7: Estimates and Odds Ratio for Year Level and Region in 2002................................136

A. R.8: Estimates and Odds Ratio for Year Level and Region in 2004................................137

A. R.9: Estimates and Odds Ratio for Year Level and Region in 2005.................................138

A. SL.1: Estimates and Odds Ratio for Year Level and School Level in 1996....................139

A. SL.2: Estimates and Odds Ratio for Year Level and School Level in 1997.....................140

A. SL.3: Estimates and Odds Ratio for Year Level and School Level in 1998......................141

A. SL.4: Estimates and Odds Ratio for Year Level and School Level in 1999.....................142

A. SL.5: Estimates and Odds Ratio for Year Level and School Level in 2000.....................143

A. SL.6: Estimates and Odds Ratio for Year Level and School Level in 2001 ......................144

A. SL.7: Estimates and Odds Ratio for Year Level and School Level in 2002 .....................145

A. SL.8: Estimates and Odds Ratio for Year Level and School Level in 2003.....................146

A. SL.9: Estimates and Odds Ratio for Year Level and School Level in 2004.....................147

A. SL.10: Estimates and Odds Ratio for Year Level and School Level in 2005...................148

A. SL.11: Estimates and Odds Ratio for Year Level and School Level in 2006...................149

A. SS.1: Estimates and Odds Ratio for Year Level and School Status in 1995 .....................150

A. SS.2: Estimates and Odds Ratio for Year Level and School Status in 1996.....................151

A. SS.3: Estimates and Odds Ratio for Year Level and School Status in 1997.....................152

A. SS.4: Estimates and Odds Ratio for Year Level and School Status in 1998.....................153

A. SS.5: Estimates and Odds Ratio for Year Level and School Status in 2001 .....................154

A. SS.6: Estimates and Odds Ratio for Year Level and School Status in 2002.....................155

A. SS.7: Estimates and Odds Ratio for Year Level and School Status in 2004......................156

A. SS.8: Estimates and Odds Ratio for Year Level and School Status in 2005 ......................157

A. SS.9: Estimates and Odds Ratio for Year Level and School Status in 2006.....................158

A. STR.1: Full Model for the Student-Teacher Ratio Effect in 1995...................................159

A. STR.2: Full Model for the Student-Teacher Ratio Effect in 1996...................................160

A. STR.3: Full Model for the Student-Teacher Ratio Effect in 1998...................................161 
A. STR.4: Final Model for the Student-Teacher Ratio Effect in 1998. .161

A. STR.5: Full Model for the Student-Teacher Ratio Effect in 1999. 162

A. STR.6: Full Model for the Student-Teacher Ratio Effect in 2000 163

A. STR.7: Full Model for the Student-Teacher Ratio Effect in 2001 164

A. STR.8: Full Model for the Student-Teacher Ratio Effect in 2002. 165

A. STR.9: Final Model for the Student-Teacher Ratio Effect in 2002. 165

A. STR.10: Full Model for the Student-Teacher Ratio Effect in 2003. 166

A. STR.11: Final Model for the Student-Teacher Ratio Effect in 2003. 166

A. STR.12: Full Model for the Student-Teacher Ratio Effect in 2004. 167

A. STR.13: Full Model for the Student-Teacher Ratio Effect in 2005. 168

A. STR.14: Full Model for the Student-Teacher Ratio Effect in 2006 169

A. SS-TE.1: The Model for the School Size (Total Enrolment) Effect in 1996 170

A. SS-TE.2: The Model for the School Size (Total Enrolment) Effect in 1997 170

A. SS-TE.3: The Model for the School Size (Total Enrolment) Effect in 1998 171

A. SS-TE.4: The Model for the School Size (Total Enrolment) Effect in 1999 171

A. SS-TE.5: The Model for the School Size (Total Enrolment) Effect in 2000 172

A. SS-TE.6: The Model for the School Size (Total Enrolment) Effect in 2001 172

A. SS-TE.7: The Model for the School Size (Total Enrolment) Effect in 2002 173

A. SS-TE.8: The Model for the School Size (Total Enrolment) Effect in 2003 .173

A. SS-TE.9: The Model for the School Size (Total Enrolment) Effect in 2004 174

A. SS-TE.10: The Model for the School Size (Total Enrolment) Effect in 2005 174

B. SSTV. 1: The Full Model for 2007 Secondary School Teacher Variables 175

B. SSTV. 2: The Other Model for 2007 Secondary School Teacher Variables 175

B. SSBV. 1: The Full Model for 2007 Secondary School Building Variables. 176

B. SSFV. 1: The Full Model for 2007 Secondary School Facility Variables 178 


\section{List of Figures}

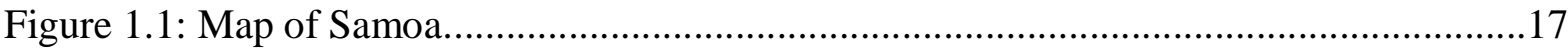

Figure 3.1: Comparing Year 2 Level dropout Rates by Region, 1995 - 2006.........................60

Figure 3.2: Comparing Year 7 Level Dropout Rates by Region, 1995 - 2006........................61

Figure 3.3: Comparing Year 10 Level Dropout Rates by Region, 1995 - 2006.......................61

Figure 3.4: Comparing Year 11 Level Dropout Rates by Region, 1995 - 2006......................62

Figure 3.5: Comparing Year 12 Level Dropout Rates by Region, 1995 - 2006......................62

Figure 3.6: Comparing Year 13 Level Dropout Rates by Region, 1995 - 2006......................63

Figure 3.7: Dropout Rate Status at Primary Level, 1995 - 2006.............................................64

Figure 3.8: Dropout Rate Status at Secondary Level, 1995 - 2006.........................................65

Figure 3.9: Comparing Year 2 Level Dropout Rates by School Status, 1995 - 2006..............66

Figure 3.10: Comparing Year 9 Level Dropout Rates by School Status, 1995 - 2006............67

Figure 3.11: Comparing Year 10 Level Dropout Rates by School Status, 1995 - 2006..........67

Figure 3.12: Comparing Year 11 Level Dropout Rates by School Status, 1995 - 2006..........68

Figure 3.13: Comparing Year 12 Level Dropout Rates by School Status, 1995 - 2006..........69

Figure 3.14: Comparing Year 13 Level Dropout Rates by School Status, 1995 - 2006..........69

Figure 5.1: Distribution of the Student-Teacher Ratio by Region (1995)..............................97

Figure 5.2: Distribution of the Student-Teacher Ratio by Region (1996)...............................98

Figure 5.3: Distribution of the Student-Teacher Ratio by School Status (2002)...................105

Figure 5.4: Distribution of the Student-Teacher Ratio by School Level (2002)...................105

Figure 5.5: Distribution of the Student-Teacher Ratio by School Status (2003)...................106

Figure 5.6: Distribution of the Student-Teacher Ratio by School Level (2003).....................106

Figure 5.7: Distribution of the Student-Teacher Ratio by School Level (2004)....................108

Figure 5.8: Distribution of the Student-Teacher Ratio by School Status (2005)...................110

Figure 5.9: Distribution of the Student-Teacher Ratio by School Level (2005)....................110

Figure 5.10: Distribution of the Student-Teacher Ratio by School Level (2006)...................112

C. RDR. G1: Comparing Yr 3 Level Dropout Rates by Region.............................................180 
C. RDR. G2: Comparing Yr 4 Level Dropout Rates by Region..........................................180

C. RDR. G3: Comparing Yr 5 Level Dropout Rates by Region.............................................181

C. RDR. G4: Comparing Yr 6 Level Dropout Rates by Region............................................181

C. RDR. G5: Comparing Yr 8 Level Dropout Rates by Region............................................182

C. SSDR. G1: Comparing Yr 3 Level Dropout Rates by School Status................................182

C. SSDR. G2: Comparing Yr 4 Level Dropout Rates by School Status..................................183

C. SSDR. G3: Comparing Yr 5 Level Dropout Rates by School Status..................................183

C. SSDR. G4: Comparing Yr 6 Level Dropout Rates by School Status.................................184

C. SSDR. G5: Comparing Yr 7 Level Dropout Rates by School Status.................................184

C. SSDR. G6: Comparing Yr 8 Level Dropout Rates by School Status..................................185

C. STR. RG1: Distribution of the Student-Teacher Ratio by Region (1997).........................185

C. STR. RG2: Distribution of the Student-Teacher Ratio by Region (1998).........................186

C. STR. RG3: Distribution of the Student-Teacher Ratio by Region (1999).........................186

C. STR. RG4: Distribution of the Student-Teacher Ratio by Region (2000)........................187

C. STR. RG5: Distribution of the Student-Teacher Ratio by Region (2001).........................187

C. STR. RG6: Distribution of the Student-Teacher Ratio by Region (2002).........................188

C. STR. RG7: Distribution of the Student-Teacher Ratio by Region (2003)........................188

C. STR. RG8: Distribution of the Student-Teacher Ratio by Region (2004).........................189

C. STR. RG9: Distribution of the Student-Teacher Ratio by Region (2005).........................189

C. STR. RG10: Distribution of the Student-Teacher Ratio by Region (2006).......................190

C. STR. SSG1: Distribution of the Student-Teacher Ratio by School Status (1995)............190

C. STR. SSG2: Distribution of the Student-Teacher Ratio by School Status (1996).............191

C. STR. SSG3: Distribution of the Student-Teacher Ratio by School Status (1997)............191

C. STR. SSG4: Distribution of the Student-Teacher Ratio by School Status (1998).............192

C. STR. SSG5: Distribution of the Student-Teacher Ratio by School Status (1999).............192

C. STR. SSG6: Distribution of the Student-Teacher Ratio by School Status (2000)............193

C. STR. SSG7: Distribution of the Student-Teacher Ratio by School Status (2001)............193 
C. STR. SSG8: Distribution of the Student-Teacher Ratio by School Status (2004)............194

C. STR. SSG9: Distribution of the Student-Teacher Ratio by School Status (2006).............194

C. STR. SLG1: Distribution of the Student-Teacher Ratio by School Level (1995)..............195

C. STR. SLG2: Distribution of the Student-Teacher Ratio by School Level (1996)..............195

C. STR. SLG3: Distribution of the Student-Teacher Ratio by School Level (1997).............196

C. STR. SLG4: Distribution of the Student-Teacher Ratio by School Level (1998)..............196

C. STR. SLG5: Distribution of the Student-Teacher Ratio by School Level (1999)..............197

C. STR. SLG6: Distribution of the Student-Teacher Ratio by School Level (2000).............197

C. STR. SLG7: Distribution of the Student-Teacher Ratio by School Level (2001)..............198 


\section{Chapter 1}

\section{Introduction}

Dropping out of school or leaving school prematurely is a problem that concerns parents, teachers and communities in many respects (Stone, 1956). It is not a problem of the present but it is a dilemma that has been happening in the past (Campbell, 1966) in every part of the world.

This study is focused mainly on the dropout crisis in Samoan schools particularly at primary and secondary level. The time frame covered in this study is from 1995 until 2007.

The main purposes of this project are to:

1) use readily available data to compare and identify the following:

- dropout rates by year level for regions (Apia Urban, Rest of Upolu and Savaii).

- dropout rates by year level for school levels (Primary, Primary-Secondary and Secondary).

- dropout rates by year level for school status (Government, Mission and Private).

2) use readily available data to identify and investigate the effect of the following variables on the school dropout rates.

- Student-Teacher Ratio

- School size

- School teachers' gender

- School teachers' ethnicity

- School teachers' qualification

- School buildings selected variables, and

- School facilities selected variables. 


\section{Research Questions and/or Hypothesis}

The study wishes to explore the following questions and/or hypothesis:

1) (i) Are students more likely to leave school early in rural areas (Rest of Upolu and Savaii regions) than the Apia urban region?

(ii) Are students more likely to drop out of higher school year levels and/or Secondary schools than lower school year levels and/or Primary schools?

(iii) Are students more likely to drop out of Government schools than Mission and Privates schools?

2) (i) Are students more likely to drop out of schools with higher Student-Teacher ratios?

(ii) Are students more likely to drop out of larger schools?

(iii) Are students more likely to drop out of schools with:

a) more female than male teachers?

b) more foreign teachers?

c) fewer teachers with degrees?

(iv) What school building variables that significantly influence the dropouts?

(v) What school facility variables that significantly associate with the dropouts?

Due to the nature of the dataset we are using for this study, we will answer hypothesis 1 (i) (iii) and 2 (i) - (ii) using the calendar years 1995 - 2006. Hypothesis 2 (iii) - (v) will be answered using the calendar year 2007 as it only has data for these variables.

\section{Structure of the Thesis}

The thesis has six chapters and each chapter is then subdivided into subsections as shown in the contents. Chapter one talks about the background information of the study and Samoa's profile as well as its education system. It also explains the Samoa Ministry of Education, Sports and Culture's Census Survey. Chapter two focuses on what the literature says about Dropouts and dropout rates. The overview of the dropout rates of Samoan schools is covered in Chapter three. Chapter four explains the Data Analysis approach and Statistical Methods used to analyse the dataset(s) for this study. The results and findings of this study will be found in Chapter five. The project is concluded with discussions and recommendations which will be covered in Chapter six. 


\subsection{Background}

This section provides an overview of Samoa as a nation in terms of its land mass, location, climate, population, history, politics, religions, culture and economy. It briefly explains the system and the status of education of Samoa. These informations were extracted and summarised from texts such as So'o et al (2006), Ministry of Education, Sports and Culture (2007a), Ministry of Education, Sports and Culture (2007b) and Ministry of Education, Sports and Culture (2006).

\subsubsection{Samoa the Nation}

\section{Land and Location}

Samoa is a group of volcanic islands which consists ten small islands. Four of these islands are inhabitant with the two larger ones sharing the majority of Samoa's population. These four islands are called Upolu, Savaii, Manono and Apolima. On the north of the island group lies Savaii with Upolu on the south while Manono and Apolima are in between the two main ones as shown by Figure 1.1 (map of Samoa). Samoa is situated south of the equator and bounded by latitudes of 13 degrees and 15 degrees south and longitudes of 168 degrees and 173 degrees west. Its capital city is Apia and is located in Upolu

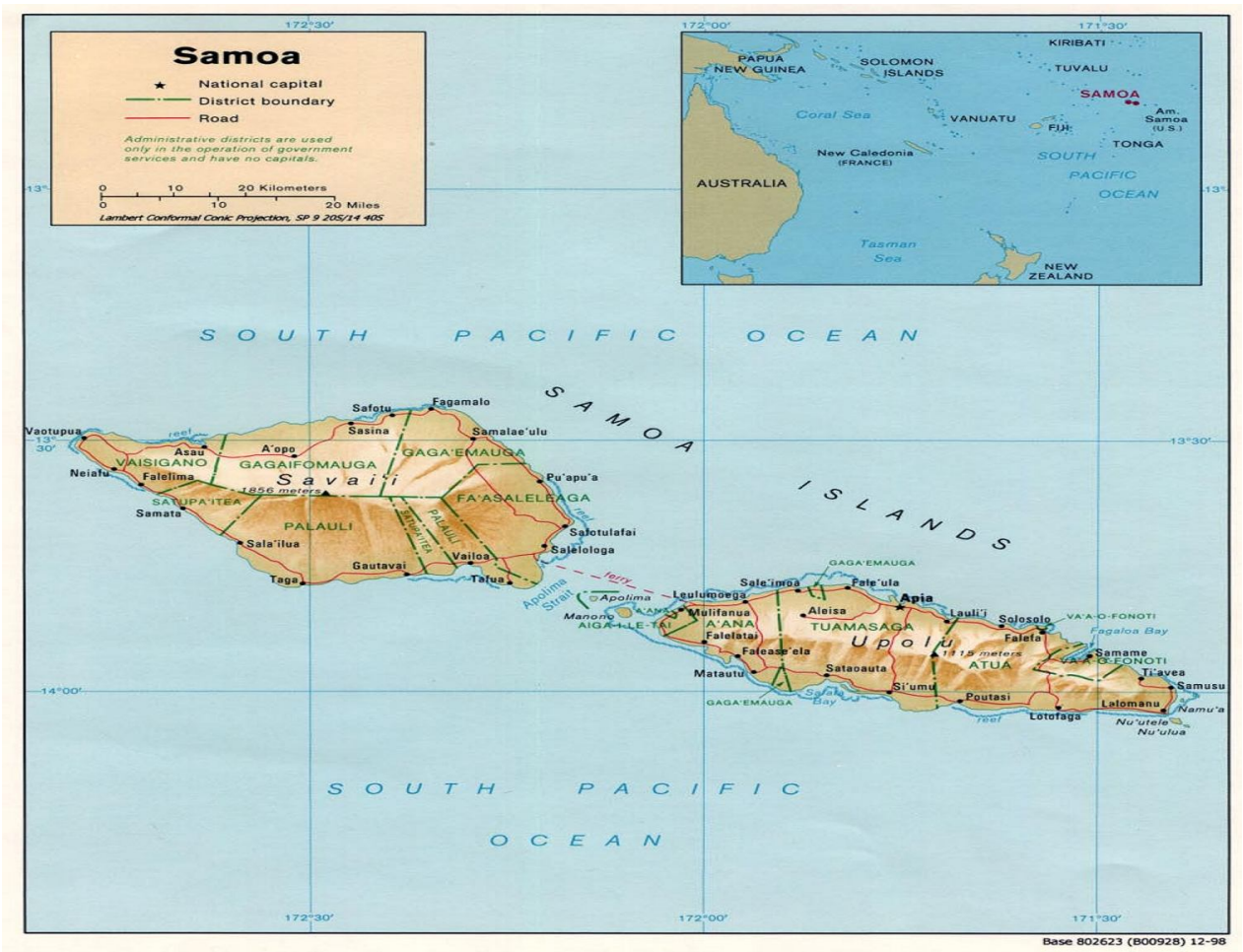

Source: www.lib.utexas edu/maps/samoa.html cited in Strategy for the Development of Samoa 2008 - 2012, 2008, p. iii

Figure 1.1: Map of Samoa 
The total land area of the country is estimated at 2,934 square kilometres with Savaii and Upolu's combined land masses add up to 2,830 square kilometres. About 19 percent of the total land mass is cultivable while 37 percent is mostly forest. According to the Samoa Bureau of Statistics, estimates of land use in Samoa, much of it is for non-merchantable forest which amounts to 43.7 percent as shown in Table 1.1. The proportions of land ownership by land type for Upolu and Savaii are shown in Table 1.2. Most of Samoan villages are situated along the coastal area where much of the flat land is.

Table 1.1: Estimates of Land Use in Samoa

\begin{tabular}{|l|r|r|}
\hline \multicolumn{1}{|c|}{ Land Use } & Areas in Hectares (ha) & Percentage (\%) \\
\hline Merchantable Forest & $18,050.2$ & 6.3 \\
\hline Communities Conservation & $1,954.0$ & 0.7 \\
\hline Watershed Areas & $44,110.0$ & 15.5 \\
\hline National Parks / Reserves & $10,495.0$ & 3.7 \\
\hline Land for Reforestation & $2,785.0$ & 1.0 \\
\hline Agriculture and Crop Land & $80,530.5$ & 28.3 \\
\hline Barren Land & $1,997.1$ & 0.8 \\
\hline Non Merchantable Forest & $124,254.2$ & 43.7 \\
\hline Total & $\mathbf{2 8 4 , 1 7 6 . 0}$ & $\mathbf{1 0 0}$ \\
\hline
\end{tabular}

Source: Samoa Bureau of Statistics: www.sbs.gov.ws

Table 1.2: Samoa Land Ownership

\begin{tabular}{|l|r|r|r|r|}
\hline \multirow{2}{*}{ Land Type } & \multicolumn{2}{|c|}{ Upolu } & \multicolumn{2}{c|}{ Savaii } \\
\cline { 2 - 5 } & Hectares (ha) & Percentage (\%) & Hectares (ha) & Percentage (\%) \\
\hline Customary & $77,087.4$ & 27.1 & $153,470.3$ & 54.0 \\
\hline Government & $18,908.3$ & 6.6 & $11,739.0$ & 4.1 \\
\hline Samoa Land & $9,485.0$ & 3.3 & $4,473.0$ & 1.6 \\
\hline Freehold & $5,630.2$ & 2.0 & $1,043.2$ & 0.4 \\
\hline Town Area & $2,429.3$ & 0.9 & & - \\
\hline Total & $\mathbf{1 1 3 , 5 4 0 . 2}$ & $\mathbf{3 9 . 9}$ & $\mathbf{1 7 0 , 6 3 5 . 8}$ & $\mathbf{6 0 . 1}$ \\
\hline
\end{tabular}

Source: Samoa Bureau of Statistics: www.sbs.gov.ws

The country's major agricultural crops are coconut or copra, taro, bananas and nonu fruit.

The means of transportation and telecommunication between and within the main islands are more efficient and very reliable. The country's international airport is located at Faleolo which is on the north west of Upolu island. 


\section{Climate}

Being located near the equator, Samoa's climate is hot and humid or simply tropical with an average temperature of 26.5 degrees Celsius and an annual rainfall of about 2,880 $\mathrm{mm}$ on average. The rainy season starts in November and ends in April each year. At this time of the year, the country always prepares for bad weather that may develop into strong winds or even cyclones.

\section{Language}

Everyone in the country speaks Samoan as their first language and English as their second.

\section{Population}

At the time of writing this thesis, the complete and final report of the 2011 Samoa Census was not available. However, according to the preliminary counts of the 2011 Samoa Census the population has increased by 3.1 percent since the 2006 census. The 2011 results show that the total persons counted were 186,340 with 96,137 (about $52 \%$ ) males and 90,203 (about 48\%) females in contrast with the 2006 results of 180,741 people of the total population with 93,552 (about 52\%) males and 86,962 (about 48\%) females. The majority of the population is found in the Rest of Upolu region $(105,100$ persons or $56.40 \%)$ while the Savaii and Apia Urban Area regions recorded 44,387 persons $(23.82 \%)$ and $36,853(19.78 \%)$ persons respectively as illustrated in Table 1.3.

Table 1.3: Total Population of Samoa in 2011 and 2006

\begin{tabular}{|l|r|r|r|r|r|c|}
\hline \multirow{2}{*}{ Region } & \multicolumn{3}{|c|}{$\mathbf{2 0 1 1}$} & \multicolumn{3}{c|}{ 2006 } \\
\cline { 2 - 7 } & Male & Female & \multicolumn{1}{c|}{ Total } & Male & Female & Total \\
\hline Apia Urban Area (AUA) & 18,576 & 18,277 & \multicolumn{1}{|c|}{36,853} & 19,120 & 18,588 & 37,708 \\
\hline Rest of Upolu (RoU) & 54,554 & 50,546 & 105,100 & 52,033 & 47,631 & 99,891 \\
\hline Savaii & 23,007 & 21,380 & 44,387 & 22,399 & 20,743 & 43,142 \\
\hline Total Population & $\mathbf{9 6 , 1 3 7}$ & $\mathbf{9 0 , 2 0 3}$ & $\mathbf{1 8 6 , 3 4 0}$ & $\mathbf{9 3 , 5 5 2}$ & $\mathbf{8 6 , 9 6 2}$ & $\mathbf{1 8 0 , 7 4 1}$ \\
\hline
\end{tabular}

Source: Samoa Bureau of Statistics: www.sbs.gov.ws

The 2011 Samoa Census classification of its population by ethnicity group was not available at the time, but the 2006 Samoa Census figures show that 97 percent of the 
total population were Samoans while the other 3\% were non-Samoans (Samoa Bureau of Statistics, 2008).

The annual population growth rate for 2006 - 2011 period, based on the 2011 preliminary figures, is 0.62 percent which is very low with the median age of 20.5 . The working population (ages 15 and over) based on 2006 final results recorded that 99 percent of the total working population were employed and the other 1 percent was attributed to unemployed ones (Samoa Bureau of Statistics, 2008). The crude birth rate for 2006 census results was calculated at 27.3 while the total fertility rate estimated at 4.2. The infant mortality rate for males and females were 18.2 and 22.9 respectively. Given these high rates of mortality at birth, the average life expectancy at birth for males and females were 71.5 and 74.2 respectively.

\section{History}

In 1899, sixty nine years after the arrivals of missionaries in the 1830s three foreign powers Great Britain, Germany and the United States of America (USA) fought over the Samoan islands. As a result Germany colonised the western part which is now known as Samoa, while USA ruled over the eastern part which was then called American Samoa up until today. In the beginning of World War One saw the surrender of the Germans control over Samoa to New Zealand. During the Second World War in 1945, the United Nations was set up and implemented its anti-colonial policies. The result of these policies saw Samoa being the first Pacific Island nation to become independent on the first of January 1962.

The island nation's name was changed from Western Samoa (as it was formally known) to Samoa on the fourth of July, 1997 under a constitutional amendment which was passed by members of the Parliament at the time. As a Pacific island nation Samoa has been relatively peaceful and stable ever since becoming independent in 1962.

\section{Political Structure}

Since 1962, Samoa adopted and implemented a western system of parliamentary democracy. From then on, only matais (chiefly titleholders) were eligible to become candidates and vote in general elections. However, this electoral system changed in the 1991 general election whereby all Samoan citizens aged 21 years and over have been lawfully and rightfully accepted to vote in parliamentary general elections. Restriction on this change allowed matais only to stand as candidates. 
The Parliament consists of the legislative assembly and the Head of State whose endorsement is required before a bill can become law. His Highness Tuiatua Tupua Tamasese Efi is the present Head of State, who was elected by the Parliament in June, 2007 as a successor to the late Head of State, his Highness Malietoa Tanumafili II for a term of five years. The legislative assembly is made up of 49 members who are elected from 41 constituencies or districts. Out of 49 members, two are representatives of non-Samoan descendants.

The speaker of the house is elected by the legislative assembly during its first meeting after the general election as well as the deputy speaker. During this session, the Prime Minister is also elected by the Parliament but most likely by the winning caucus or party. He/She then selects 12 members of his/her Cabinet from the Parliament. Under the constitution, the judiciary is independent and it comprises of the Chief Justice and the President responsible for the criminal court system and the Land and Titles court system respectively.

At the moment the governing and also the dominant party in Samoan politics is the Human Rights Protection Party (HRPP) which came into office in 1988 (Ministry of Education, Sports and Culture, 2007a), the longest serving party in power.

\section{Religion and Culture}

Religion is very important to Samoan people as 99.7 percent of the population are Christians. The main denominations consist of Congregational Christian Church of Samoa (34.8 percent), Roman Catholic (19.6 percent), Methodist (15 percent), Latter Day Saints (12.7 percent), Assembly of God (6.6 percent) and Seventh Day Adventists (3.5 percent) (Ministry of Education, Sports and Culture, 2007a). The other denominations share 7.8 percent of the population among themselves. So'o et al, (2006) identify other denominations such as Jehovah's Witness ( 0.8 percent), CCCJS (1.0 percent), Nazarene ( 0.4 percent), Protestant ( 0.2 percent), Baptist $(0.2$ percent), Full Gospel (0.8 percent), Voice of Christ ( 0.4 percent), Worship Centre (1.3 percent), Peace Chapel (1.3 percent), Anglican Church (0.2 percent), Community Church (0.3 percent), Elim Church (0.1 percent), Samoa Evangelism (0.1 percent), Aoga Tusi Paia or Bible Study (0.4 percent) and Bahai (0.5 percent).

Despite the strong association of Samoan people with their religions, it would not weaken the bond between them and the Samoan culture. The Samoan culture shapes the way of life of its indigenous people which is known as the faaSamoa. This has been truly reflected in each village and the way villagers live their lives, particularly in rural areas. Each village is run by the chief system and comprised of a group of extended families. The matai (chief) is the head of each family and is elected by older people of the family through a process called talanoaga (family meeting). The matai can be a male or female who represents the family at the village council (fono). At the 
village council, matais discuss village matters and are responsible for making laws and punishments to guide and control the village. The Pulenuu (village mayor) who is also a matai has a term of three years and is responsible for ceremonial duties.

\section{The Economy}

Samoa's economy is relatively small but steady in comparison to other small Pacific island nations. It depends heavily on agriculture, foreign aids and remittances from families and relatives overseas such as Australia, New Zealand and the United States of America.

The driving force of the country's economic growth in the mid 1990 was fisheries, agriculture and tourism. This economic growth rate put Samoa in a very strong position which was then reduced from 5.2 percent in 2005 to 2.6 percent in 2006. This was caused by the declining of fishing, agriculture, construction activities and Yazaki production. However, at the end of 2006 saw a 9.1 percent increase in tourism earnings and a 12 percent rise in tourists' arrival.

Samoa's largest export is fish, followed by nonu juice, beer and coconut cream. These products contributed to the country's total export earnings of SAT28.75 million in 2006 (Ministry of Education, Sports and Culture, 2007a).

Samoa's currency is 'Tala' which is abbreviated to SAT meaning Samoan Tala.

\subsubsection{National Education Context}

Education in Samoa has come a long way even before the arrival of missionaries in the nineteenth century. The traditional and cultural teaching and learning process through storytelling and role modelling have helped develop the education system of Samoa. The establishment of Pastors' schools in the villages was also recognised nationwide from then until today. The impact of the Germans and New Zealand's colonisation of the island in 1899 until mid-1990s brought modern changes to the education context of the nation at the time. Thus the current status of the Samoa education system through MESC is a far cry from where and what it was before.

Today the Education system of Samoa is subjective under the Education Ordinance 1959, and the Youth, Sports and Culture Affairs Act 1993/1994 (Ministry of Education, Sports and Culture, 2007a). Under this Act it clearly prescribes the 
MESC's mission "to promote and encourage the development and improvement of all phases of education, sports and culture in Samoa".

\section{Goals of Education}

The goals of education in Samoa are congruent with the global goals of Education For All (EFA), the Millennium Development Goals (MDGs), the Forum Basic Education Action Plan (FBEAP), the Pacific Plan and the Decade of Education for Sustainable Development (DESD) (Ministry of Education, Sports and Culture, 2006). These goals include the quality improvement at all levels of education, achievement of universal primary education, expand and improve early childhood education, improve adult literacy and access to life skills and continuing education for adults and youth, eliminate gender disparities in schools and achieve gender equity, and poverty reduction, development of good governance, elimination of disease, and achievement of environmental sustainability (Ministry of Education, Sports and Culture, 2006).

\section{Aims of Education}

The Ministry of Education, Sports and Culture has identified five key elements which are the basis of its policies and practices for nine years, starting from July 2006 to June 2015. These five key concepts were extracted from the Ministry of Eucation, Sports and Culture (2006), and are summarised below.

1. Equity requires the system to treat every individual fairly and equally in terms of services and opportunities.

2. Quality is focused on improving and attaining high levels of academic achievement, cultural understanding and sensitivity and social participation.

3. Relevancy is when the system is meaningful, recognised, applicable and useful to one's life. This is visualised as all learning that are relevant and appropriate to individual, community and national development.

4. Efficiency requires the use of effective management through human resources including teachers, financial and material resources at all levels, timely and quality service delivery and sufficient and satisfactory facilities.

5. Sustainability requires transparency and accountability at all levels. It also requires the wise utilisation of adequate resources such as human, financial and material resources to ensure balanced and continual development in the system. 


\section{Education Policies and Strategies (2006 - 2015)}

According to the Ministry's Strategic Policies and Plan's (2006 - 2015) vision, effective policy, planning and the quality of research and accurate information analysis will help improve and boost the Ministry's performance. This is basically the main responsibility of the Policy, Planning and Research Division (PPRD) within the Ministry. This Division is compromised of Research and Policy Officers, Planning Officers, Information Analysis Officers and Projects Coordinator Officers. They are the ones, who conduct research, coordinate, develop and inform policies and plans, monitor specific projects and gather and analyse information.

The main focus of this Division is to strengthen areas of weaknesses in policy development, research, planning, and information analysis and project coordination. In addition, the use of variety of methods in data collection and analysis will also be considered and improved.

\section{Management of Education}

The review of the 1995 to 2005 Education Policies and Strategies identified weaknesses in the management and administration of education which needed to be addressed (Ministry of Education, Sports and Culture, 2006). As a result, Institutional Strengthening Programmes (ISP) through the Ministry of Education at the time were implemented starting from 1999. The main focus was to provide support and build institutional capacity of the Ministry by formulating policies and procedures as well as manuals and guidelines to improve operations and management. This process of professional development of corporate staff and teachers has been going on for quite a while and there has been an increase in opportunities for professional development.

The latter may result from merging the Department of Education and the Sports and Culture sections of the Ministry of Youth, Sports and Culture to form the Ministry of Education, Sports and Culture. The merging took place in 2003.

\section{The Core Executive}

The executive panel is chaired by the Chief Executive Officer (CEO) who is supported and assisted by six Assistant Chief Executive Officers (ACEOs). Each ACEO heads each of the six divisions known as School Operations (SOD); Curriculum, Materials and Assessment (CMAD); Policy, Planning and Research (PPRD); Corporate Services (CSD); Sports (SD); and Culture (CD). These officers plus the CEO of the Samoa Qualifications Authority (SQA), the Education Consultant, the Project Manager of the Education Sector Project II 
(ESPII) and the Dean of the Faculty of Education (FOE) of the National University of Samoa (NUS) form the MESC's Core Executive (Ministry of Education, Sports and Culture, 2006).

The full executive is the combination of the Core executive and all those at the Principal Officer level.

\section{School Review Officers}

There are twenty one (21) educational school districts in the country and each of them has a School Review Officer (SRO) who acts as a manager at the district level. The responsibility of the SRO is to make sure, monitor and report the implementation of curriculum policies, assessment, teaching, student and staff administration, school management and other issues in relation to the students, teachers or the school to the Ministry.

The list of the twenty one educational districts is found in Appendix F.

\section{School Management}

According to the Ministry of Education, Sports and Culture (2006) the school principal is responsible for the overall ruling of the school with the assistant of the school committee. All school committees are provided with extensive trainings about school management and school improvement. The deputy or the vice principal takes over the ruling of the school if or when the principal is not present.

\section{Status of Education in 2007}

There were 204 schools in 2007. The Rest of Upolu region recorded the highest number of schools in it which is $94(46.08 \%)$ as shown in Table 1.4. 64 schools (31.37\%) were located in Savaii and the other 46 (22.55\%) were found in the Apia Urban region. Table 1.4 also shows the distribution of these schools into three school status Government, Mission and Private by region. There were 166 Government schools $(81.37 \%)$, followed by 30 Mission schools $(14.71 \%)$ and 8 of them were Private schools $(3.92 \%)$. The 30 Mission schools were shared amongst 6 control authority while 8 Private schools were shared amongst 2 control authority as shown in Table 1.5. 
Table 1.4: Number of Primary and Secondary schools for each Educational Region by School Status

\begin{tabular}{|l|c|c|c|c|}
\hline \multirow{2}{*}{ Region } & \multicolumn{3}{|c|}{ School Status } & \multirow{2}{*}{ Total } \\
\cline { 2 - 4 } & Government & Mission & Private & \\
\hline Apia Urban & 25 & 13 & 8 & $\mathbf{4 6}$ \\
& $(54.35 \%)$ & $(28.26 \%)$ & $(17.39 \%)$ & \\
\hline Rest of Upolu & 84 & 10 & 0 & $\mathbf{9 4}$ \\
& $(89.36 \%)$ & $(10.64 \%)$ & $(0.00 \%)$ & \\
\hline Savaii & 57 & 7 & 0 & $\mathbf{6 4}$ \\
& $(89.06 \%)$ & $(10.94 \%)$ & $(0.00 \%)$ & \\
\hline Total & $\mathbf{1 6 6}$ & $\mathbf{3 0}$ & $\mathbf{8}$ & $\mathbf{2 0 4}$ \\
& $(\mathbf{8 1 . 3 7 \% )}$ & $\mathbf{( 1 4 . 7 1 \% )}$ & $\mathbf{( 3 . 9 2 \% )}$ & \\
\hline
\end{tabular}

Table 1.5: The Distribution of schools into Control Authority by School Status

\begin{tabular}{|l|c|c|c|c|}
\hline \multirow{2}{*}{ Control Authority } & \multicolumn{3}{|c|}{ School Status } & \multirow{2}{*}{ Total } \\
\cline { 2 - 4 } & Government & Mission & Private & \\
\hline Baptist & 0 & 3 & 0 & $\mathbf{3}$ \\
& $(0.00 \%)$ & $(100 \%)$ & $(0.00 \%)$ & \\
\hline C.C.C.S & 0 & 6 & 0 & $\mathbf{6}$ \\
& $(0.00 \%)$ & $(100 \%)$ & $(0.00 \%)$ & \\
\hline Catholic & 0 & 12 & 0 & $\mathbf{1 2}$ \\
& $(0.00 \%)$ & $(100 \%)$ & $(0.00 \%)$ & \\
\hline Government & 166 & 0 & 0 & $\mathbf{1 6 6}$ \\
& $(100 \%)$ & $(0.00 \%)$ & $(0.00 \%)$ & \\
\hline L.D.S & 0 & 4 & 0 & $\mathbf{4}$ \\
& $(0.00 \%)$ & $(100 \%)$ & $(0.00 \%)$ & \\
\hline Methodist & 0 & 3 & 0 & $\mathbf{3}$ \\
& $(0.00 \%)$ & $(100 \%)$ & $(0.00 \%)$ & \\
\hline Peace Chapel & 0 & 0 & 2 & $\mathbf{2}$ \\
\hline S.D.A & $(0.00 \%)$ & $(0.00 \%)$ & $(100 \%)$ & \\
\hline School Board & 0 & 2 & 0 & $\mathbf{2}$ \\
& $(0.00 \%)$ & $(100 \%)$ & $(0.00 \%)$ & \\
\hline \multicolumn{1}{|c|}{ Total } & 0 & 0 & 6 & $\mathbf{6}$ \\
& $(0.00 \%)$ & $(0.00 \%)$ & $(100 \%)$ & \\
\hline
\end{tabular}

Table 1.6 reveals the distribution of the 204 schools into three school types Boys only, Co-education and Girls only by school region. Two hundreds of all schools were Coeducation, 1 Boys only and 3 Girls only. 
Table 1.6: The Distribution of schools into Regions by School Type

\begin{tabular}{|l|c|c|c|c|}
\hline \multirow{2}{*}{ School Type } & \multicolumn{3}{|c|}{ School Region } & \multirow{2}{*}{ Total } \\
\cline { 2 - 4 } & $\begin{array}{c}\text { Apia } \\
\text { Urban }\end{array}$ & Rest of Upolu & Savaii & \\
\hline Boys only & 1 & 0 & 0 & 1 \\
& $(100 \%)$ & $(0.00 \%)$ & $(0.00 \%)$ & \\
\hline Co-education & 42 & 94 & 64 & $\mathbf{2 0 0}$ \\
& $(21 \%)$ & $(47 \%)$ & $(32 \%)$ & \\
\hline Girls only & 3 & 0 & 0 & $\mathbf{3}$ \\
\hline Total & $(100 \%)$ & $(0.00 \%)$ & $(0.00 \%)$ & $\mathbf{2 0 4}$ \\
\hline
\end{tabular}

There is three type of school level in Samoa and they are Primary, Primary-Secondary and Secondary. However, the Primary-Secondary level is given to schools that cover both Primary and Secondary and are located within the same compound. There were 7 schools under this category in 2007. The allocation of all schools by these levels is found in Table 1.7.

Table 1.7: The Distribution of schools into School Status by School Level

\begin{tabular}{|l|c|c|c|c|}
\hline \multirow{2}{*}{ School Status } & \multicolumn{3}{|c|}{ School Level } & \multirow{2}{*}{ Total } \\
\cline { 2 - 4 } & Primary-Secondary & Primary & Secondary & \\
\hline Government & 1 & 141 & 24 & $\mathbf{1 6 6}$ \\
& $(0.60 \%)$ & $(84.94 \%)$ & $(14.46 \%)$ & \\
\hline Mission & 5 & 13 & 12 & $\mathbf{3 0}$ \\
& $(16.67 \%)$ & $(43.33 \%)$ & $(40.00 \%)$ & \\
\hline Private & 1 & 6 & 1 & $\mathbf{8}$ \\
& $(12.50 \%)$ & $(75.00 \%)$ & $(12.50 \%)$ & \\
\hline Total & $\mathbf{7}$ & $\mathbf{1 6 0}$ & $\mathbf{3 7}$ & $\mathbf{2 0 4}$ \\
& $(\mathbf{3 . 4 3 \% )}$ & $\mathbf{( 7 8 . 4 3 \% )}$ & $(\mathbf{1 8 . 1 4 \% )}$ & \\
\hline
\end{tabular}

\section{Primary Education}

Primary education is compulsory for all students ages 5 to 14 . This school level covers an eight year cycle that starts from Year 1 to Year 8 (Ministry of Education, Sports and Culture, 2007a). Students have the right to enter either into a Government, Mission or Private school. When the student reaches the end of his/her final year of primary schooling, he/she requires to sit the Year 8 National Examination to determine entry into secondary schools. 
All Government primary schools are village owned and managed by school committees. The school committee is responsible for school buildings, facilities, such as equipments and furniture, and collection of school fees. The Ministry provides and pays school teachers, school stationery and curriculum resources, assessment, examinations, teachers' in-service training and development (Ministry of Education, Sports and Culture, 2006).

In 2007 , there were 39,578 students enrolled in 160 primary schools throughout the nation as in Table 1.8 (Ministry of Education, Sports and Culture, 2007a). There were $30(14.71 \%)$ schools in the Apia Urban region, 79 (38.73\%) in the Rest of Upolu region and $51(25 \%)$ for the Savaii region as shown in Table 1.9. $51.77 \%$ of the Primary enrolment comes from Males and $48.23 \%$ is Females as shown by Table 1.8. Further, $83.40 \%$ of the Primary enrolment is from Government schools while $12.56 \%$ is from the Mission schools and only 4.04\% comes from Private schools.

Table 1.8: Primary Schools 2007 enrolment by School Status

\begin{tabular}{|c|c|c|c|c|}
\hline \multirow[t]{2}{*}{ Enrolments } & \multicolumn{3}{|c|}{ School Status } & \multirow[t]{2}{*}{ Total } \\
\hline & Government & Mission & Private & \\
\hline Males & $\begin{array}{c}17,232 \\
(84.10 \%)\end{array}$ & $\begin{array}{c}2,449 \\
(11.95 \%)\end{array}$ & $\begin{array}{c}808 \\
(3.94 \%)\end{array}$ & 20,489 \\
\hline Females & $\begin{array}{c}15,778 \\
(82.65 \%)\end{array}$ & $\begin{array}{c}2,521 \\
(13.21 \%)\end{array}$ & $\begin{array}{c}790 \\
(4.14 \%)\end{array}$ & 19,089 \\
\hline Total & $\begin{array}{c}33,010 \\
(83.40 \%)\end{array}$ & $\begin{array}{c}4,970 \\
(12.56 \%)\end{array}$ & $\begin{array}{c}1,598 \\
(4.04 \%)\end{array}$ & 39,578 \\
\hline
\end{tabular}

Table 1.9: Number of Schools in Samoa by School Level in 2007

\begin{tabular}{|l|c|c|c|c|}
\hline \multirow{2}{*}{ Region } & \multicolumn{3}{|c|}{ School Level } & \multirow{2}{*}{ Total } \\
\cline { 2 - 4 } & Primary-Secondary & Primary & Secondary & \\
\hline Apia Urban & 3 & 30 & 13 & $\mathbf{4 6}$ \\
& $(6.52 \%)$ & $(65.22 \%)$ & $(28.26 \%)$ & \\
\hline Rest of Upolu & 2 & 79 & 13 & $\mathbf{9 4}$ \\
& $(2.13 \%)$ & $(84.04 \%)$ & $(13.83 \%)$ & \\
\hline Savaii & 2 & 51 & 11 & $\mathbf{6 4}$ \\
& $(3.13 \%)$ & $(79.69 \%)$ & $(17.19 \%)$ & \\
\hline Total & $\mathbf{7}$ & $\mathbf{1 6 0}$ & $\mathbf{3 7}$ & $\mathbf{2 0 4}$ \\
& $(\mathbf{3 . 4 3 \%})$ & $\mathbf{( 7 8 . 4 3 \% )}$ & $(\mathbf{1 8 . 1 4 \%})$ & \\
\hline
\end{tabular}




\section{Secondary Education}

Secondary education or schools cater for all students who have completed their Year 8 level. It covers a five year cycle that starts from Year 9 to Year 13. Students' entrance into secondary schools is determined by the Year 8 National Examination whereby top achievers go to Samoa College (one of the Government secondary schools) in Apia. The next group go to Vaipouli College (one of the Government secondary schools) for Savaii students and Avele College (another Government secondary school) for Upolu students. The rest of the students go into secondary schools or colleges within their respective district (Ministry of Education, Sports and Culture, 2006).

Like primary schools, the Ministry provides and pays school teachers, school stationery and curriculum resources, assessment, examinations, teachers' in-service training and development (Ministry of Education, Sports and Culture, 2006). The school committees are responsible for other things that the Government through the Ministry does not provide which include fundraising.

There are two major examinations that students are required to sit while in secondary schools. The National School Certificate examination is sat at the end of Year 12 to determine entrance into Year 13 level. Those who pass and achieve required marks proceed to Year 13 while others may repeat Year 12 or else. The Pacific Senior Secondary Certificate (PSSC) examination is sat at the end of Year 13. The outcome of this examination determines the entrance of a student to the Foundation an equivalent of Form 7, which is run by the National University of Samoa (NUS).

In 2007, there were 15,165 students enrolled in 37 secondary schools throughout the country as shown in Table 1.7. 24 of which were Government schools, 12 were Mission schools and 1 was a Private school. According to Table 1.9, there were 13 secondary schools in the Apia Urban region as well as for the Rest of Upolu region and 11 were from the Savaii region.

\subsection{Ministry of Education, Sports and Culture Survey}

The Ministry of Education, Sports and Culture through the PPRD has been conducting the Samoa Education Census every year particularly at primary and secondary schools for educational, national and statistical purposes. 


\section{Objective(s)}

Data collection through the Ministry's Census Form will help achieve its goals and aims as mentioned in section 1.1.2. This is simply about achieving better opportunities for all learners in Samoa.

Thus the information collected will help the Ministry to formulate plans and strategies for the betterment of the education system in the country. This information is also submitted to the Parliament and the United Nations Education, Science and Culture Organisation (UNESCO) for statistical purposes.

\section{Description of Survey}

Every year, the Ministry of Education, Sports and Culture through PPRD carries out a survey on primary and secondary schools nationwide. The survey is called the Census whereby both Government and Non-Government primary and secondary schools are involved. The Early childhood education, special education, vocational and tertiary education are addressed by separate surveys.

The PPRD is responsible for preparing everything for the survey. This includes the Principals' checklist, the SRO checklist, booklets and the Census Forms. The preparation is done in February every year before distributing the forms to each school for completion. Each school must get one copy of the Census form which has 37 standard questions in it. The completion of the census within the school must be conducted in an organised and efficient way. However, it is recommended by the Ministry that the principal is responsible for completing the census form for small schools as stated in the Census Form (see Appendix D). He/She is also responsible for any forms that have been given to teachers to complete to check and make sure that they are completed accurately. In large schools the principal may delegate census duties to the deputy-principal (or equivalent) plus the assistance of the teachers to fill in the census forms and return them to the principal for final check.

The Census day is held on the first Wednesday of March every year and may take a week or two to complete the census forms and return them back to the Ministry on the assigned date given in the form for analysis. There is a validation period of two weeks after the Census date to double check forms for missing and/or incorrect information. Forms with incorrect and/or missing information will be sent back to the responsible schools to correct and fill in the gaps.

The delivery of the census forms to each school is done through the School mail system which goes out every week. For Savaii schools they receive their mails on Tuesdays and Wednesdays while Upolu schools get theirs on Tuesdays, Wednesdays and Thursdays. For both Government and Private schools, the census form must be 
delivered directly to the school principal while for Mission schools it must be received by the Education Director. The returning of the census forms back to the Ministry is the responsibility of either the SRO or the school principal for Government and Private schools. For Mission schools the Education Director gets to drop it off at the Ministry on the assigned date.

The completed and accurate census forms are entered into the Ministry's database called MANUMEA.

\section{The 2007 Survey}

For the 2007 survey the Ministry of Education, Sports and Culture through PPRD carried out the Census survey on all primary and secondary schools nationwide.

The PPRD prepared and organised everything for the survey. This includes the Principals' checklist, the SROs' checklist, booklets and the Census Forms. The preparation and organisation was done in February 2007, before delivering the forms to each school for completion. There was one Census form for each school and it was delivered through the school mail system. There were 204 forms packed in sealed white and brown A4 envelopes that were sent out to schools and returned back to the Ministry of Education, Sports and Culture.

The census form shown in Appendix D consisted of 37 standard questions. Section one was about general information about the school and it contained 20 questions. Section two was about students' enrolment and their age of all level and it covered questions 21 and 22. The Primary level enrolment and classes was the main focus of section three and question 23. Section four and question 24 was about the secondary level enrolment and classes. Section five through questions 25 and 26 recorded the number of students being absent on the census day for both primary and secondary level respectively. The last section was section six and it contained questions 27 to 37 about school facilities and assets.

The Census was held on the $7^{\text {th }}$ of March, 2007. There was a validation period of two weeks after the Census date to double check forms for missing and incorrect information. Forms with incorrect and missing information were sent back to the responsible schools for correction.

\section{The Database}

The completed and accurate census forms were entered into the Ministry's database called MANUMEA. This database is an access type application and is used for the 
Ministry's Census data entry. It is responsible for preparing and printing out of reports in forms of Statistical Digest from the Census.

\section{Data Coding and Data Entry}

Each school was given its own code alphabetically generated by the database. Therefore the coding of the census forms was done exactly the same way relative to the school code. This process was done before distributing the forms to schools for completion.

Upon arrival of Census forms from all the schools, the PPRD body checked and stored them into boxes before entering into the database.

The Data entry process was done by the PPRD officers. The team was lead by the Principal Education Officer for Information Analysis, Mr Sooalo Mene Siaosi. He was assisted by the Principal Education Officer for Research and Policy Analysis, Mrs Quandolita Reid-Enari, and the Executive Assistant for PPRD, Mrs Ruta Afemata.

After the data entry all forms were stored and put away in Ring binders by Educational school districts.

\section{Dissemination of Information}

Once the data was entered into the MANUMEA database, then it became Public information in forms of Statistical Digest. The latter consisted of figures, graphs and tables only for the public's interest. Reports in this document could be used by Government Ministries, Co-operations, Schools, Donors, Politicians, Stakeholders or even the Public.

The 2007 MESC Annual Report to the Legislative Assembly of Samoa included a section with comments and interpretations about figures, graphs and tables on school statistics.

\section{The Datasets}

The original dataset consists of 17 separate Excel worksheets including information as follows:

- Primary Schools Age Distribution 
- Primary Schools Total Enrolment

- Schools Dropout Rates by year level

- Schools dropout and transition rates calculations

- Schools Facilities

- Secondary Schools Enrolment by Gender

- National Dropout Rates

- National Transition Rates

- Secondary Schools Repeaters

- School Buildings

- All Schools

- School Teachers

- Secondary School Classes

- Secondary Schools Age Distribution by Gender

- Secondary Schools Total Enrolment

- School Transition Rates, and

- Primary Schools Enrolment by Gender

The files were merged together using SAS. 


\section{Chapter 2}

\section{Literature Review}

Academic achievement of students is a status for school performance and an amenity to quality education regardless of the level of education it is. In most cases, the academic reputation of the school can sometimes depend on it and when there are obstacles in the way, the quality of education is affected and the efficiency of the system is questioned. One of the obstacles to students' education is leaving schools prematurely, quitting schools, giving up schools or dropping out of schools. Dropping out of school appears to be one of the major concerns at the national level (Christenson \& Thurlow, 2004). The dropout problem is neither an educational virus of the new era nor a problem of yesterday. According to Campbell (1966), it did not just begin. Historically it dates back to the beginnings of education. He also emphasized that it is not a problem of today's generation but it has been a problem of the past generations.

The dropout problem is like an infected wound that takes time to heal, unless it needs proper treatment and care which will eventually heal it. As Tyler \& Lofstrom (2009) state; "dropping out is as hard to prevent as it is easy to do". Further, if we can not take necessary measures and remedial actions to prevent it, leaving school prematurely will be as worse as an unsolved mystery and "becomes a greater one in the future" says Campbell (1966).

This chapter will focus on the review of some of the findings that some of the researchers, politicians, educationists and even the media have reported on, regarding those students who drop out of school and the factors associated with it. The literature is abound with definitions of dropping out of school, which primarily is the focus of section 2.1 Factors that are associated with the dropouts or the dropout rates and reasons why students are quitting schools are explained in section 2.2. The geographical locations or regions of these early school leavers (Hattam \& Smyth, 
2003) and the dropout status in these regions will be the subject of section 2.3 , to be followed by school level or level of education and the dropout status in section 2.4. In section 2.5 the study will draw attention on the school status or school sectors of these dropouts and the dropout status, whereas student-teacher ratio as well as school size impacts on the response variable will be collectively discussed in section 2.6. Nevertheless, the impact of school buildings and school facilities on withdrawing from school is the main focus of section 2.7 while section 2.8 details the aftermath of giving up school early and possible interventions and initiatives in preventing this educational virus from spreading. This review of the literature will be summed up with a conclusion in section 2.9.

\subsection{The Global Definition of Dropout or Dropout Rate}

Numerous definitions of dropout and the lack of a common definition stems from the different ways of collecting the dropout data. In most countries, the educational definition of dropout is in a form of a rate which is called the dropout rate, as most of the education administrative data are collected through census forms. For this reason, the dropout rate (DR) is then calculated as 100 subtracts both the promotion rate (PR) and the repetition or retention rate (RR). For example, the Ministry of Education, Sports and Culture (MESC) in Samoa calculates the dropout rates by year level (grade) based on the enrolment figures, including the repeaters and those promoted to the next year level, collected from every school through census forms annually. The definition of dropout rate in the Samoan context is given in chapter 3 , and it is referred to as:

"the percentage of students who neither progressed from one level to the next nor repeat the level" (Ministry of Education, Sports and Culture, 2007).

This definition adopted by UNESCO (2006) is the "proportion of pupils who leave the system without completing a given grade in a given school year". This definition of dropout is quite common internationally, especially for least developing countries including the Pacific nations as it was also reported by other studies such as the Education Support Program (ESP), (2007). The formula that is used by MESC to calculate its dropout rates is the same as the one used by UNESCO. This calculation is also used by the Ministry of Education of Ethiopia. According to the Ministry of Education of Ethiopia (2010), the dropout rate is a measure typically by grade, of those who leave formal schooling which is the residual of those who repeat and promoted to the next grade from enrolment. Another study in primary education, about the participation of Ethiopian female students in school by Nekatibeb (2002), has adopted that of Loxley (1987) which is defined as: 
"Dropouts are those who leave school before the end of the final year of an education cycle in which they are enrolled". (Nekatibeb, 2002, p.4)

However, Allensworth (2005) saw it in a different way, whereby in her article she reiterated the definition used by Alexander, Entwisle \& Horsey (1997) and Finn (1989).

"Dropout is a result from weak attachment to school developed through years of poor school performance and feelings of failure”.

Allensworth (2005) definition is similar to that of Christenson \& Thurlow (2004) of which they argue that students' decision to drop out is an outcome of a prolonged process of disengagement from school. In their paper on prevention considerations, interventions and challenges for school dropouts, they refer to disengagement from school as poor attendance, unsuccessful school experiences both academic and behaviour, feelings of alienation, poor sense of belongings and a general dislike of school. According to Rumberger \& Palardy (2005), their definition of dropping out of school is very simple and expresses as a rate; whereby dropout rate is referred to as " $a$ percentage of students who quit school before completion". It seems like Rumberger \& Palardy's (2005) definition is a summary of Rumberger's (2001) definition of which he specifically viewed dropout "as a status or persons who have not completed high school and who are no longer enrolled in school or program that can lead to high school completion at a particular point in time". By and large, this definition of dropout basically overarches all the other definitions as mentioned earlier. Stan van Alphen (2009) in his research of the dropout crisis across Europe, he found out that in European countries the common definition for early school leavers or so-called dropouts are those who failed to complete upper secondary education.

However, the definition of dropping out of school in the United States of America (USA), according to Chapman, Laird, Ifill \& KewalRamani (2011), is a little bit different and very particular. With their definition they have expressed it as a rate by categories both at state and national level. The first category is called the event dropout rate (Chapman, Laird, Ifill \& KewalRamani, 2011) which is the proportion of high school students (both public and private) who quit high school between the beginning of a school year and the beginning of the next without attaining a high school diploma or an equivalent qualification. The other category is more focused on the percentage of students of a specific age group. This definition is known as the status dropout rate (Chapman, Laird, Ifill \& KewalRamani, 2011) which is the proportion of students in a specific age group who are out of school (both public and private) and have not attained a high school diploma or an equivalent qualification. These two clarified definitions of the dropout rate in the United States are similar to that of Warren \& Jenkins (2005), of which they used in their analyses of the dropout situation in Florida and Texas. However, theirs is based on individuals rather than a rate. They argue that for a given year, dropouts are those who were not enrolled in school in October of that year but were enrolled in school in the same month of the previous year, and reported that their highest completed level of schooling was the 
$9^{\text {th }}, 10^{\text {th }}$, or $11^{\text {th }}$ grade. But according to Campbell (1966) his definition of an early school leaver or dropout is one of the most detailed and explicit ones and seems like he is arguing from a wholistic point of view. He clearly claims that a dropout is an individual who does not complete the twelve grades of school for reasons other than sickness, death, transfer to other schools, being sent to correctional facilities, or expel from school. He also emphasises that such individual may belong to any educational level, socio-economic background, geographical regions, and perhaps demographic background.

In a monitoring study of the dropout situation in six of the Middle-East and European countries by the Education Support Program (ESP), (2007), the dropout definition varies across all these nations. Language barriers and translation difficulties were the obvious reasons reported of why the team in this monitoring program employed different definitions. In their final report they discovered that the Organisation for Economic Co-operation and Development (OECD) (2002) defines a dropout student as an individual who quits a specific level of education without gaining an academic qualification. Nevertheless, the study also rediscovered one of the well-known definitions by Morrow (1987):

A dropout is any student previously enrolled in a school, who is no longer actively enrolled as indicated by fifteen days of consecutive unexcused absences, who has not satisfied local standards for graduation, and for whom no formal request has been received signifying enrolment in another statelicensed educational institution. A student death is not tallied as a dropout. (ESP, 2007, pp 17)

We believe that Morrow's definition is kind of similar to that of Campbell (1966) as mentioned earlier. Despite the availability and the clarity of these international definitions of dropping out of school, the ESP (2007) team tended to re-define it in a very simple and meaningful way that fits into the countries under study contexts. For example, in Latvia the team used two definitions:

a) A dropout is an individual who has left the education process early and no longer enrolled in school and has not completed basic education.

b) Individuals on the brink of dropping out are those who have been abstained from school for six months and thus not being able to complete basic education.

However, in Slovakia the team just simply defined dropouts as those who have not completed basic education. In addition, a dropout is an individual, who quits school before completion for any reason other than death and has not reenrolled in another school or institution, is the definition that best suited Albania's context.

Discrepancies in defining the terminology when the study was in Kazakhstan, as there were two agencies responsible and accountable for collecting information on dropouts, and each one had its own term. For instance, the Ministry of Education in 
Kazakhstan defines dropouts as those who miss school for at least 10 days while the Ministry of Internal Affairs describes these school leavers as those who miss school frequently without any valid excuse.

More versions and a lack of a common definition were also discovered in Mongolia eventhough the official description of the terminology is referred to students who refrained from going to school after attending it for a while. These versions depend on who is defining it. For example, the Ministry of Education in Mongolia describes the situation as students at the age of compulsory education (currently 7 to 16 years old) who are not attending school. But for local school officials' understanding, dropouts are those who have never enrolled in the school before and those who have not completed secondary school. Local school teachers as well, also have their side of the story which by and large expands the school officials' definition. With their version, they argue that dropouts are individuals who have never attended school or have a history of unexcused absences and are removed from the list or simply, those who just quit school. The study also pointed out that it did not stop there at the level of teachers in digging up views and opinions toward the dropout definition. The team also had to hear from both the parents and children of Mongolia and here is what they collectively thought of dropouts:

"dropouts are those who have neither secondary education nor secondary higher education". (ESP, 2007, pp.18)

Despite the different and colourful versions of dropping out used by the team, they have reported that all of the six countries participated came into terms and utterly agreed to use a wider and broaden definition. This definition claims that:

"school dropouts are those in the compulsory school age who are either not in school at the end of the school year, not completing the last grade at the compulsory level, not registered for the new school year, or have not received a certificate of education for their respective age group" (ESP, 2007, pp 19).

Overall, we gather from these various definitions that dropping out of school is simply referring to a process or a pathway of disengagement from school at any time without any qualification, of which students or individuals of any level of education, from different geographical locations, with different socio-economic status and of different demographic backgrounds go through or take as their last resort, due to environmental pressure such as school and academic factors as well as social and family factors. Dropouts are the endproducts of this process. For this study we will stick to the dropout rate definition provided by the Samoan Ministry of Education, Sports and Culture, which is given in section 3.1. 
The study wanted to find out why Samoan students drop out of school but the dataset we are using does not have any information on individuals to reflect these factors. Hence this section identifies some of the factors associated with the dropout from the literature which may be related to Samoan school dropouts.

Without doubt, students prematurely give up school due to many factors which really affect their academic ability. Some of these factors largely contribute to students' decision to quit school while some have minor effects or no effects at all. But on top of the range is poverty ( $\mathrm{Yi}$, et.al, 2011) which is one of the common reasons why students are dropping out of school. They have found that higher dropout rates in China especially in rural areas are associated with poverty. This is also supported by Fischer (2010) in her study of immigrants' educational performance in the United States of America. She claims that immigrants living in a household below poverty line are more likely to drop out than those living in a household above the poverty line. A similar finding was also found in another study by Fetler (1989) cited in the North Carolina Department of Public Instruction (2000). Further, Provasnik et al, (2007) reports that higher status dropout rate for individuals of aged 16 to 24 in rural (11.1\%) America was those below poverty level (23.2\%). Further more, Lofstrom (2007) too in his investigation of student-level data from the Texas School Microdata Panel (TSMP), believes that poverty was one of the ingredients which caused high dropout rates for Hispanic students. Lofstrom's (2007) argument is strongly verified by Johnson, Strange \& Madden (2010) that higher dropout rates in all 616 rural school districts in 15 of the States, are caused by high poverty particularly for Hispanic and African-American students. From the other side of the world to the other, ESP (2007) and UNICEF (2008) both affirm that poverty is the main reason why Albanian students are departing schools early. In addition ESP (2007) also reports that this is also the main reason for students to give up school in other countries like Kazakhstan, Latvia, Mongolia, Slovakia and Tajikistan. Crossing the Pacific Ocean Malin \& Meidment (2003) believe that Indigenous students of Australia are most likely to drop out of school due to poverty. But in Fiji Naidu, Barr \& Seniloli (2007) reported that a considerable number of school dropouts are from families with poverty. They indicate that the situation has become worst in the country recently which saw $65 \%$ of dropouts are from these families. The same reason for Fijian students to quit school is also reported by Singh (2008), as part of the Fijian Interim Minister of Education's speech delivered at the $101^{\text {st }}$ Fiji Principals' association conference, in his media article for the Fiji Times. Nevertheless, poverty is quite common in most of SubSaharan African countries such as Ethiopia according to Admassu (2011). In his findings from the 2004 national welfare monitoring survey (WMS) of Ethiopia, he pointed out that poverty is the major factor for Ethiopian students to leave school early as families can not afford it. He found that higher dropout rates were experienced in the poorest household as compared to those in the wealthiest level. 
Poverty could be one of the reasons for some of the Samoan students to quit school as some of the families, especially in the rural areas, are poor.

Another common factor that is associated with dropping out of school is opportunity cost (Yi, et.al, 2011). Students' academic ability are severely affected by this factor which sees them finding jobs to earn money to help out with the family as parents can not afford to financially send them to school. In the case of China, Yi et.al (2011) argue that high wages in unskilled labour lured many secondary school students into these jobs which induced them to quit school. This point is supported by Meekers \& Ahmed (1999) in their study of 4,368 women aged 15 to 49 in Botswana. They reported that school girls who dropped out of school were more likely from femaleheaded families, due to male labour migration to South Africa which consequently leaving these single and uneducated women jobless. This would result in very hard for these single women to pay for their children's education. A study by Ilon \& Moock (1991) also revealed that higher opportunity costs in terms of child labour income and low income for rural families in Peru were not enough to sustain children's education. The latter was also the case for Australia according to Hattam \& Smyth (2003), based on their study of 209 young school leavers through interviews. They indicate that most students give up schools due to family having no money. The severeness of opportunity costs sees other families in the Netherlands, especially with those of single-headed and have four or more children (Tanja Traag \& Rolf K. W. van der Velden, 2011), unable to send their children to school. But according to ESP (2007), the situation for the six countries participated in the study becomes worse. For example, in Albania about 32\% children at the age between 5 and 14, and about $60 \%$ of males at the age of 10 and above left school early and worked to help their families. In addition, about $8 \%$ children in the sample worked permanently and about $30 \%$ worked occasionally. Furthermore, of the 200,000 children dropping out of schools in Mongolia since 1990, most of them were working to contribute to their families' income. The impact of this factor on dropouts becomes worse in Fiji as indicated by Naidu, Barr \& Seniloli (2007). They say that despite the free tuition for students up to senior secondary schools, families still can not afford to send their children to school due to other educational costs, and as a result quit school and employ in very low wage employment to help support the family (Naidu, Barr \& Seniloli, 2007). Similar finding was also discovered in Veramu's (2002) paper on 'extending the reach for schools' in Fiji as well. This is one of the reasons identified by So'o et al (2006) why some of the Samoan students drop out of schools as well as the socio-economic status of the student's family.

The socio-economic status of the family is one of the most common factors associated with the dropout as indicated by Tyler \& Lofstrom (2009), which is also supported by a study on school dropout preventions by Christenson \& Thurlow (2004). These two studies both indicate that higher dropout rates are amongst those from low-income backgrounds. On the same note Lee \& Burkam (2003) also highlight this association in middle schools in America which Meekers \& Ahmed (1999) also report it for the 
case of Botswana. Another study by Ilon \& Moock (1991) mentions that female students from low income families are more likely to give up school, which in fact verifies by Rumberger (2001). The latter argues that higher dropout rates were discovered for families with low income as compared to those with middle and high income. Lamb (2011) has emphasized the same point in his paper on TVET and the poor: challenges and possibilities; whereby he mentions that early school leavers in Australia are more likely from low socio-economic families. Tanja Traag \& Rolf K. W. van der Velden (2011) agrees to Lamb's findings when they mention the same reason why the Netherland's students drop out of school. According to much of the literature, the problem of having low income or financial constraints or costs (Wils, 2004; Vosamana, 2012; Veramu, 2002; Ilon \& Moock, 1991) seems to be an ongoing issue that hinders and jeopardises students' academic ability.

Other common factors that have been reported by the literature that are associated with dropping out include those of the family, school and even students themselves. For the family factors, students are more likely to quit school due to family stability (Tyler \& Lofstrom, 2009) which reflects in school mobility or transfer (Christenson \& Thurlow, 2004; Lee \& Burkam, 2003; Rumberger \& Palardy, 2005; ESP, 2007; Branham, 2004; Tyler \& Lofstrom, 2009). All of these authors consistently report that higher dropouts were discovered in schools with higher transfer rates particularly immigrant students (Fischer, 2010) of ethnicity minority and for students whose families have changed residence (Rumberger \& Palardy, 2005) due to other reasons apart from refugee (ESP, 2007). To shed some more lights on higher dropout rates for immigrant students, Rumberger (2001) claims that more Hispanic students dropping out of school compared to other ethnicity minorities, is due to the fact that $40 \%$ of them were born overseas, and more than $40 \%$ of these overseas born never attended school in the United States. This underpins Fischer's (2010) findings in which she shows that immigrant students who have just arrived in the States are more likely to become victims of dropping out than those who have been there a long time ago. In addition, she also finds that those who arrived in the States after the age of 9 are about 4 times more likely to quit school, while those who arrived before the age of 9 are about 2 times higher than that of the American citizens.

Other students drop out of school because of other family problems (Barrowman, Nutbeam \& Tresidder, 2001) such as divorce, alcohol use, lack of parental support and overcrowded households (ESP, 2007). The latter includes families with more number of siblings and as indicated by Admassu (2011), children of such families especially older girls, are more likely to leave school early. Furthermore, some students give up school due to either their fathers lost their jobs or mothers got sick (Hattam \& Smyth, 2003). The latter is supported by Admassu (2011) where he found that respectively, a student is about 4 and 3 times more likely to drop out of school both in rural and urban areas in Ethiopia, when a family member is sick. Sadly the odds of dropping out for these students, especially rural areas, increasingly rise to $33 \%$ when someone in the family dies (Admassu, 2011). 
It has been proven by research that school factors are arguably as one of the push outs (Yi, et.al, 2011; Rumberger \& Palardy, 2005; ESP, 2007) for students especially those who are at the verge of dropping out. Lee \& Burkam (2003) in their investigation of 3,840 students from 190 urban and suburban high schools in America, argue that higher dropout rates are caused by school factors. These school factors such as cost and access can seriously affect students' school attendance particularly in rural areas (Ilon \& Moock, 1991). With regards to school teachers, very often parents invest a lot on their children's education in order to get good outcomes at the end, but only to find lower returns in their educational investment due to poor quality of teachers (Yi, et.al, 2011). This has been proven significantly and positively associated with dropout rates by Tyler \& Lofstrom (2009) and other researchers in America, like Lee \& Burkam (2003) where they claim that schools with more excellent teachers have lower dropout rates. However, Rumberger \& Palardy (2005) did not find any significant relationship between dropping out and teacher quality; although they indicated that the attitude of teachers such as efficacy has positive impact on dropout rates, and high expectation for student learning, has impinged on dropout rates. They also claim that leadership skills of both school principals and teachers have significant impact on students especially those who are at risk.

The quality of teachers is also reflected in their attitude and their social relationship with their students. According to ESP (2007), many ethnicity minority (particularly Roma students) and low achieving students, dropped out of schools due to teachers' negative attitudes towards them. Moreover, it has been reported that $23 \%$ of street students were those who quit school because teachers were making fun of them. Such negative and punitive or repressive attitude of teachers consequently discourage (Malin \& Meidment, 2003) students from attending schools. Nevertheless, it also causes students to feel unsafe (Rumberger \& Palardy, 2005; Hattam \& Smyth, 2003), alienated (Ream \& Rumberger, 2008; ESP, 2007) and embarrassed (Malin \& Meidment, 2003) and later quit school. Furthermore, it creates negative relationship (Hattam \& Smyth, 2003; Barrowman, Nutbeam \& Tresidder, 2001; Rumberger, 2001) between these teachers and at risk students. Not all teachers have attitude problems as Lee \& Burkam (2003) indicate in their study, where students are less likely to drop out of schools where the student-teacher relationship is positive. This is also supported by Pittman (1991) in which he argues that a positive social relationship between students and staff reduce dropout rates. This positive relationship could be an outcome of such schools having employed very experience teachers (Branham, 2004), or a result of some education policies whereby schools, parents and the community working together to improve the quality of teaching, reports Gahna News Agency (GNA) (2012).

Speaking of policy, some studies show that some government and school policies have negative influence on dropping out of school whereas some studies find some policies inefficient. For example, Ilon \& Moock (1991) argue that despite education 
reforms to improve students' attendance in schools in Peru, rural areas still have higher dropout rates. They also claim that rural schools are the last receivers of these policies and are the first to get affected. But for most of the Sub-Saharan countries in Africa like Botswana, education policy for pregnant school girls disallow them from attending schools until their child reaches one year old (Meekers \& Ahmed, 1999). In the same study, some school policies force these pregnant teenagers to continue their education in another school elsewhere as they are not allowed back anymore. It appears that school policies hold the power to exclude students from school through suspension or expulsion (Malin \& Meidment, 2003) as in the case of the Aborigines in Australia. Other school policies like compulsory exit exams for students see many of them sadly disengage from the education system and have hesitated to go back. These exit exams such as high stake tests (Tyler \& Lofstrom, 2009) may lower the completion rate and at the same time are more likely causing students to think twice about attending school again. Students of ethnicity minority and from rural areas are more likely to be affected by these exit exams. For example, Yi, et.al (2011) report that China has a competitive exam based education sytem particularly for junior high school students, if they want to promote to senior high schools or colleges. If rural students do not pass these exams, then they are more likely to accumulate the dropout rate as they are disallowed to enrol in any of those senior high schools or colleges. This is also supported by Christenson \& Thurlow (2004) in which they argue that such school exit exams may increase the dropout rate, which is also the concern of Rumberger \& Palardy (2005) for American at risk or low achieving students. These low academic students are eliminated by school external exams (Veramu, 2002) and become members of the dropout family in Fiji. However, Warren \& Jenkins (2005) did not find any significant relationship between dropping out of school and high stake exams in both Texas and Florida. But they did mention that there are other factors that they were not aware of as why the association was not significant. They also indicate that proponents of exit exams insist that these exams encourage students to work harder and assist educationists to identify and correct weaknesses in the education system. For example, in 1980s high school students were required to pass exit exams in order to obtain regular diplomas (Warren \& Jenkins, 2005). On the other hand, other schools use exit exams to push out low level achieving students in order to gain a good reputation for the school (Yi, et.al, 2011) while other schools use exams to indentify weaker students and transfer them to alternative programs so they are not regarded as dropouts (Rumberger \& Palardy, 2005). In Samoan schools there are high stake exit exams such as Year 8 National and Year 12 School Certificate that students sit. If a student fails then he/she will not progress to the next year level (grade) which as a result is either repeat the same year level or drop out.

Other researchers suggest that for those who fail these exams can be held back (Lofstrom, 2007) or repeat the same grade or year level. Some believe that retention is like a second chance opportunity for students to work harder, while teachers and parents give more support for them to be able to complete school and have high level of academic achievement. For example, Ministry of Education, Ethiopia (2010) 
reports that repetition rates are higher for boys than girls of grade 8 due to policy requirement for these students to repeat before resitting exit exams, even though they say that more resoures will be allocated for these repeaters than others and all go to wastage when some of these students give up school later on. They also mention that overall repetition rates are very low in Ethiopia but higher in dropout rates. But according to Allensworth (2005) retention can be as dangerous as exit exams as it adds more fuel to the fire. She constantly insists that failing exams and grade retention both increase the likelihood of dropping out by lowering the students' self confidence, combined feelings of failure, negative attitudes against the school and being old in the class. Rumberger \& Palardy (2005) in their findings claim that retention during grade 1 to grade 8 have largely affected students' learning ability and in turn increase the dropout rate. Ilon \& Moock (1991) also argue that many rural children in Peru repeat grades more frequently but then later fall into the valley of early school leavers. The odds for these repeaters to be ended up into these valleys are 35\% more likely than those who stay on track (Lofstrom, 2007). Other education and school policies are very efficient in reducing the dropout rates such as liangmian yibu (Yi, et.al, 2011), known as two waivers, one subsidy. This policy is about waiving junior high school students' fees and tuition in order to achieve higher completion rates, and reduce dropout rates in China, which officially became law in 2006 (Yi, et.al, 2011). Since the implementation of the new policy, dropout rate has dropped from $8 \%$ to less than $2 \%$ in 2007.

Very often people are very quick to accuse school factors other than family factors as reasons why students disengage from schools, but sometimes students themselves have to get the blame for failing schools. This is similar to what Rmberger \& Palardy (2005) have argued in their analysis of the 1988 National Education Longitudinal Survey in the United States. They say that the differences of school outcomes are not because of schools' inefficiency but also consider students' characteristics. According to Ream \& Rumberger (2008) about half a million American students leaving school early, and one third of these students are high school students who fail to graduate due to lack of participation in school activities (both academic and extra curricular) (McNeal, 1995; Ma, 2003), which leads to poor or low school performance. Students with low school performance or lower level of academic achievement have lower level of sense of belongings and lower self esteem too (Ma, 2003). Such students with attitude problems can frequently cause serious behavioural problem like harassment and racism (Hattam \& Smyth, 2003; Malin \& Meidment, 2003; ESP, 2007) and even drinking alcohol, smoking cigarettes and marijuana and engage in premarital sex (Barrowman, Nutbeam \& Tresidder, 2001; Veramu, 2002) at school which is a concern for rural teachers in the States (Provasnik, et.al, 2007). Other students just quit school due to lack of motivation, or lost interest and even feelings of boredom (ESP, 2007). Schools that offer mainly academic courses and few non-academic courses (Lee \& Burkam, 2003) could be one possibility of why these students are getting bored at school. 
Researchers also report that some students leave school prematurely is because of health problems that they are having (ESP, 2007; Ma, 2003; Barrowman, Nutbeam \& Tresidder, 2001) and late enrolment (Ilon \& Moock, 1991; Wils, 2004). Wils (2004) claims that late school entrants in Mozambique are more likely to leave school early than those who enrolled on time. She finds that those who first attend school at the age 5 to 7 are more likely to survive til grade 8, while those who just enter at the age 11 to 14 will manage to survive as far as one quarter of the grades of their younger counterparts. Other factors such as pregnancy (Tyler \& Lofstrom, 2009; Meekers \& Ahmed, 1999) while at school and distance between the students' residence and schools (Ilon \& Moocks, 1991; ESP, 2007; Singh, 2008; Wils, 2004; Admassu, 2011) also affect the students' academic ability in the expected direction.

For pregnancy, Meekers \& Ahmed (1999) sadly report that the majority of school girls in Botswana and other Sub-Saharan African countries drop out of schools due to childbearing or pregnancy. In their investigation of a sample of 4,368 women aged 15 to 49 , about $7 \%$ of these women involved in sexual relationships before turning 15 , while about $54 \%$ only engaged in sexual intercourse between the age of 15 and 17 and about $22 \%$ just lost their virginity at the age 18 and 19 . Moreover, about $24 \%$ of young mothers aged between 15 and 19 have at least one child already and about $47 \%$ of those age 19 at the time were either having a child or pregnant. They also claim that with an increasing rate of schoolgirls' pregnancy, which consequently accumulates the dropout rate, could be resulted from the disappearance of traditional ways of socialization and lack of or minimum parental supervision over youth's sexual behaviour. Some of these pregnant school girls are willing to continue their education but because of strict school policies on pregnancy, they will either give up school or opt for abortion as they are frightened of expulsion (Meekers \& Ahmed, 1999). Pregnant school girls are also found in Samoan schools and like Botswana schools, these girls are more likely to give up schools.

For the distance between the students' homes and schools, Ilon \& Moocks (1991) argue that most of rural children in Peru leave school early due to high costs of transportation, because schools are quite far from where these children live. This is also in aligning with the findings by ESP (2007) on the six countries from the Middle East and Europe. Nevertheless, Singh (2008) in her media report for Fiji Times of Mr Filipe Bole's (Fiji's Minister of Education) address in which she reports that distance to schools is one of the reasons why Fijian students quit school. The long distance problem expands to African countries like Mozambique (Wils, 2004) and Ethiopia (Admassu, 2011). For example, it has been reported by Wils (2004) that respectively in 1997, about $18 \%$ and $89 \%$ of Mozambique's population lived at least $5 \mathrm{~km}$ away from a primary school and a secondary school. But according to Admassu (2011), Ethiopian students live as far as 3 to $5 \mathrm{~km}$ from school have about $70 \%$ more chance of withdrawing from school than those live nearby. Furthermore, if a student lives at least $5 \mathrm{~km}$ away from the school then the likelihood of dropping out is even larger. 
This could be one of the reasons why Samoan students drop out of school as some students live far away from the school.

\subsection{Geographical location and Dropout or Dropout Rate}

The dropout problem is not really confined to a very particular area or region. It happens all over the place either in urban, suburban or even rural areas as indicated by many researchers. According to Yi, et.al (2011) the status of the dropout rate in rural areas in China is much higher even government policies such as liangmian yibu (as mentioned in section 2.3) are not enough to solve it. It increases from $13 \%$ in the late 1990 s to an average of $40 \%$ several years later. But Lee \& Burkam (2003) in their analysis of data collected from a sample of 3,840 students from both urban and suburban high schools in America, they claim that very high dropout rates were discovered in urban areas than suburban. They reaffirm their discovery by indicating that about one third of large cities' students who enrolled in grade 9 left high school earlier than expected. These findings are verified by Provasnik, et.al (2007) in 2004 where the status dropout rate for those aged 16 to 24 was higher in the cities (12.8\%) than both in rural (11.1\%) and suburban (9\%) areas. From their analysis we could also see that a high proportion of high school students was found in rural areas as compared to suburban areas. This high proportion is attributable to a very large number of teenagers in rural areas who are neither enrolled in school nor employed (Provasnik, et.al, 2007). In addition, Lofstrom (2007) also supports these findings as well. He says that students who attend schools in central large and mid-size cities are more likely to drop out of school than any other location, especially Hispanic and African-American students. However, Johnson, Strange \& Madden (2010) reveal their findings based on 616 rural high schools in 15 Southern and Southernwest states in America; whereby higher dropout rates and lower graduation rates were found in these rural high schools due to poverty, and very little amount of fund received by these schools. The same trend for this region is also reported by Chapman, Laird, Ifill \& KewalRamani (2011), whereby nationally status dropout rates were higher for the South and the West regions ( $8.4 \%$ and $8.6 \%$, respectively) than other regions in the States.

Apart from the United States of America, the dropout problem in other part of the world is as miserable as that of the USA and very alarming too. For example, a study of 2,500 households in rural Peru by Ilon \& Moock (1991) reveal rural children quit school at a much younger age due to late enrolment and other reasons mentioned in section 2.3. The dropout rate is higher in rural areas as many of these dropouts started school at a base level compared to their counterparts in the urban areas. But in Canada, Ma (2003) in his investigation on 6,883 grade 6 students from 148 schools and 6,868 students from 92 schools in one of the rural provinces, New Brunswick, 
claims that students with lower level of academic achievement and sense of belongings are more likely to drop out. Many of these students are grade 8 lads who may also have lower self esteems and poor health conditions. In comparison to Albania, UNICEF (2008) reported that rural areas have higher dropout rates than urban areas because, there were about 5\% $(14,000)$ unenrolled children found during the time of the survey. For those in school only $8 \%$ of them were from poor families compared to $28 \%$ of wealthiest children and only $90 \%$ of these in-school students complete the four year of compulsory education. In the same report, Roma children are most likely to depart school early as only $48 \%$ of them enrolled in primary schools, and only $25 \%$ managed to complete and it reduces to about $4 \%$ for those who progressed to secondary schools. ESP (2007) reports that Roma children in Slovakia are 30 times more likely to drop out, 14 times more likely to repeat and 5 times more likely to get very low marks.

In the Netherlands, higher dropout rates are found in extremely urban areas (Tanja Traag \& Rolf K. W. van der Velden, 2011) than moderately urban areas, which cause the odds of leaving school early to be as high as about 2 times. This could be a result of having a considerable large numbers of ethnicity minority students in an extremely urban school than moderately urban school. In Fiji, Vosamana (2012) argues that students from rural areas and outer islands dropped out of school due to financial burdens, which is the same reason why the dropout rates are higher for rural areas in Mozambique (Wils, 2004). But in Ethiopia, despite the improvement in the dropout rate over the years, Admassu (2011) identifies that primary school students in rural areas are 3 times more likely to drop out than their peers in the urban areas. Moreover, students from female-headed family in both urban and rural areas are 58\% and $46 \%$ more likely to attend school than their counterparts from male-headed families respectively.

In sum the dropout status varies across geographical locations in different countries. However, it is very apparent based on evidents presented by researchers in this section that for economically stabled, wealthy and developed nations the dropout rate is higher in very urban areas than suburban and rural areas. But for economically poor, unstabled and least developing countries the dropout rate is much higher in rural areas than in urban areas.

\subsection{School Level and Dropout or Dropout Rate}

The context of school level in this study refers to the level of education such as primary, secondary or junior high and high schools or even colleges. From the literature, different countries have different terms for classes or levels. For examples, 
in USA and other western nations they call it grade(s) while in Samoa and other Pacific nations they call it year level(s).

A study by Yi, et.al (2011) shows that dropout rates grow with grades and ages. That is the higher the grade or the older the student is the higher the dropout rate. What they have found is that about $6 \%$ dropped out of school between grade 7 and grade 8 while $9 \%$ left school prematurely between grade 8 and grade 9 . They have also indicated that about $14 \%$ dropped out as early as before the end of grade 9 which is about 6 times higher than the officially recognised 3-year total junior high school dropout rate of about $3 \%$. This is also proven by Chapman, Laird, Ifill \& KewalRamani (2011) whereby the event dropout rate is much higher $(19.1 \%)$ for those aged 20 to 24 years old, compared to $2.8 \%$ and $2.5 \%$ for those aged 15 to 16 and 17 years old respectively. Similar trend was also found in the status dropout rate. The same pattern is also presented in Christenson \& Thurlow (2004) and McNeal (1995) for high schools situation in America. They have reported that the rate for an American high school to leave school early is unbelievably 1 in every 9 seconds, in contrast to 1 in 8 students never gradute from high school. The number of students who drop out of high school without graduating could increase to at least a million every year (Tyler \& Lofstrom, 2009). There was one year where the number of these high school students dropping out has reached 37 million (Rumberger, 2001). Lofstrom (2007) believes that the increasing number of high school dropouts and lower graduation rates (Ream \& Rumberger, 2008) are attributable to larger proportions of both Hispanic and African-American students than white students in America. Rumberger \& Palardy (2005) have identified some of the reasons as mentioned in section 2.3 why the dropout rates are so high in American high schools.

In African countries like Botswana, pregnant school girls contribute to more than 50\% of dropouts at secondary schools (Meekers \& Ahmed, 1999). They also claim that about $8 \%$ of these pregnant school girls dropped out of primary schools and about $20 \%$ left secondary schools prematurely due to being pregnant. According to the Ministry of Education, Ethiopia (2010) the dropout rate is as higher as about $23 \%$ in grade 1 than in grade 7, especially boys. Another study of the Primary school and dropout in Ethiopia by Admassu (2011) support these findings. He too claims that students are more likely to drop out at grade 1 and grade 8 than any other grade in between. He adds that higher dropout rates for grade 1 is probably due to new school entrants are trying to familiarise and adjust to the school environment. This is also supported by Wils (2004) where she finds out that higher dropout rates are found in the first two grades of primary education in Mozambique. She also indicates that the dropout rates fall in middle grades then rise up again in higher grades. However, Nekatibeb (2002) points out that higher female dropout rates in both of the largest regions in Ethiopia, Amhara and Oromia, are those aged between 14 and 19 years old.

On the other hand, Ilon \& Moock (1991) discover that $12 \%$ of primary students had never enrolled in schools while $16 \%$ were currently out of school in rural Peru. But 
promising figure by ESP (2007) indicates that $10 \%$ of primary school students in all the six countries participated including Albania is out of school. They also indentify a considerable unmber of unenrolled students, students who occasionally attend schools and those who quit school before the end of the compulsory education period. But according to UNICEF (2008), attendance and participation rates, enrolment and completion rates for Albanian students are very low but higher dropout rates at secondary school than at primary schools. Similar trend is also applied to Indigenous students in Australia, where most of teenagers aged between 15 and 19 years old give up school before reaching Year 12 (Malin \& Meidment, 2003). In the Netherlands, boys are 2 times more likely to leave lower secondary school early than girls (Tanja Traag \& RolfK. W. van der Velden, 2011). While in Fiji, almost 17,000 secondary school students aged between 15 and 24 leave school every year (Veramu, 2002) and only a few of them are able to get a job. In addition Vosamana (2012) reports that of those who dropped out of schools almost $50 \%$ of them did not complete high school, while $2 \%$ failed to reach class 6 . But it was even worse four years ago where only $15 \%$ of primary school children completed the full eight years of primary education, and of those who survived about 75\% successfully make it to Form 6 and Form 7 (Singh, 2008).

To summarise the school level dropout situation it looks like, despite the variations of dropout patterns in different countries, the dropout rate is much higher in higher level of education. This means that older students in higher grades tend to have very slim chances of surviving high school cycle, due to several factors as mentioned in section 2.3, and finally give up school for good.

\subsection{School Status and Dropout or Dropout Rate}

The context of school status in this study refers to either government or public schools in general, or mission schools as in catholic schools, or private as it is also referred to as independent schools.

During 2008 to 2009 school year, Chapman, Laird, Ifill \& KewalRamani (2011) reports a considerable large number (about 607,000 or equivalent to $4.3 \%$ ) of public high school dropouts alone in all 50 states in the USA. However, they also indicate that overall, about $3.4 \%$ public and private high school students who enrolled in October 2008 did not complete schools before October in the following year. Higher dropout rates for public high school students are also endorsed by Rumberger \& Thomas (2000) cited by Lee \& Burkam (2003). They argue that public schools have higher dropout rates than both catholic and private schools. However they later discover that when a Catholic school student is being transferred to a public school, 
then he or she will find himself or herself to be more likely to be an additional member of the dropout tribe. Furthermore, they also claim that Non-Catholic private schools have lower dropout rates but higher transfer rates than public schools, which Rumberger \& Palardy (2005) also agree to it. In a similar study by Lubienski \& Lubienski (2006) in their study on comparing mathematical achievements between public and private school students, they demonstrate that private school students have higher level of achievement than public school students.

Apparently it appears like whatever happens in the United States also occurs in other countries such as Australia. According to an interviewed study of 209 young dropouts by Hattam \& Smyth (2003), they argue that high-class private school students are less likely to drop out than public school students. Public school students who are from wealthiest or highly and well educated families are disaffected and are able to survive the dropout crisis.

From what we have discovered in this section we could conclude that public schools (or government schools in the Samoan context) have higher dropout rates than either private or Catholic schools (or Mission schools in the Samoan context) due to reasons already mentioned in section 2.3.

\subsection{Student-Teacher Ratio and School Size Impact on Dropout or Dropout Rate}

Much has been said about the impact of the quality of teachers on dropout rates as witnessed earlier in section 2.3. Many researchers also discuss the effect of the quantity of teachers, as in student-teacher ratio, on dropping out of school. But because the student-teacher ratio depends on the school enrolment or the size of the school, thus school size may also associate with the dropout rate. Having said that, higher student-teacher ratio means that student enrolments or class sizes are larger and fewer teachers, whereas lower student-teacher ratio indicates student enrolments or class sizes are smaller or low.

The student-teacher ratio is believed to be one of the education indicators to measure the efficiency and the quality of the education system (Ministry of Education, Ethiopia, 2010). The lower this ratio is the better the opportunity is for students to get in touch with the teachers and hence a better teaching-learning process (UNESCO, 2006; Ministry of Education, Ethiopia, 2010). On the other hand very low ratio indicates an inefficient use or under utilisation of teachers which may lead to low efficiency (Ministry of Education, Ethiopia, 2010).

According to Tyler \& Lofstrom (2009), schools with higher student-teacher ratio also have higher dropout rates. In other words, schools with lower dropout rates are a 
result of having lower student-teacher ratio (Lee \& Burkam, 2003). Similar findings by Rumberger \& Palardy (2005) underpin this, where they claim that schools with fewer number of teachers (or higher student-teacher ratio) have higher transfer and attrition rates which in turn have higher dropout rates. In another similar study by Lofstrom (2007), he also discovers that higher dropout rates also significantly associate with the student-teacher ratio in the positive direction.

However, Branham (2004) disproves these findings by indicating that lower studentteacher ratio does not necessarily increase the attendance rates or equivalently, decrease the dropout rates. He also suggests that other factors such as the teaching experience (Lee \& Burkam, 2003, Ilon \& Moock, 1991) of teachers should also take into consideration. Ilon \& Moock (1991) also argue in support of Branham's claim, that for rural schools in Peru not only they have higher dropout rates but they too have lower student-teacher ratios. This is also clearly presented, black and white, in Provasnik, et.al (2007) findings of the status of education in rural America; whereby higher dropout rates in rural areas are largely attributable to public schools that have lower student-teacher ratio. They also find that only $23 \%$ of teachers in rural areas compared to $34 \%$ in suburban areas. This could pose a question of why the studentteacher ratios are lower in other schools or regions but higher in other schools or regions. One reason for sure is the teacher salary. UNICEF (2008) reports that many school teachers in Albania look for high paying jobs to earn a living, as teachers' salaries are significantly lower than national average wages. It is the same reason why many teachers in Peru rarely teach in rural areas as salary gaps between rural and urban areas are significantly different (Ilon \& Moock, 1991). This is also the case for public school teachers in rural America. Provasnik, et.al (2007) discloses that in 2003 to 2004, on average rural public school teachers' salary is less (US\$43,000) than their peers in town (about US\$46,000), suburbs (US\$45,700) and cities (US\$44,000). They also uncover that fewer highly qualified teachers work in rural areas had masters degree or higher as compared to their counterparts in suburban areas. Rumberger \& Palardy (2005) also argue that schools with higher teacher salaries have lower dropout rates. According to Ministry of Education, Ethiopia (2010), the student-teacher ratio for primary schools is 50 and 40 for secondary schools. These figures maybe too high for other countries or too low for other countries but still, despite the successful student-teacher ratio reductions in Ethiopia to meet their millennium development goals, the dropout rates are still unrealistically high.

The school size in terms of enrolment is another factor associated with the dropout rate or dropping out of school. It is assumed that the larger the school size the higher the dropout rate or conversely, the smaller the school size the lower the dropout rate. This assumption or hypothesis has been exposed by Tyler \& Lofstrom (2009) where students are more likely to drop out of large high schools in the States. Rumberger \& Palardy (2005) also reveal the similar findings and in addition they say that small schools are more efficient than mid-size schools (between 600 and 1,200 students) in terms of promoting student learning. Another study by Pittman \& Haughwout (1987) and Branham (2004) prove that larger schools are more likely to have higher dropout 
rates. On the same note, Lee \& Burkam (2003) argue that any school less than 1,500 students are more likely to be disaffected by the dropout fever, which in turn underpins Ruberger \& Thomas (2000) claim cited in their study. However, Provasnik, et.al (2007) reveal that with regards to higher dropout rates in rural public schools, many of these public school students attend very small schools with a total enrolment less than 200. Literally, schools with smaller enrolments or fewer students are more likely to be the recipients of higher dropout rates. This disproves all the findings given by some researchers used by this study. Lastly, Pittman (1991) argues that the association between the school size and dropout are by and large impacted by other random factors.

In summary, based on these findings, both the student-teacher ratio and the school size are significantly associated with the dropout rate in both directions. That is, as indicated by some researchers that some schools with higher student-teacher ratio also have higher dropout rates, while some schools (including Samoan schools) with higher student-teacher ratio have lower dropout rates and vice versa.

\subsection{School Buildings and School Facilities Impact on Dropout or Dropout Rate}

The study believes that school buildings and facilities or resources are also associated with dropping out of school. Yi, et.al (2011) also concern about this as they argue that higher dropout rates are caused by poor quality of school facilities. This is supported by Naidu, Barr \& Seniloli (2007) and Singh (2008) where they both claim that a disproportionate large number of affected students or dropouts in Fiji are because of lack of resources and poor quality of infrastructure. The poor quality of infrastructure of school facilities may refer to small classrooms, shortage of chairs and desks, lack of drinking water and toilets (Admassu, 2011) and benches and blackboards (SAPPROS Nepal, 2011) to name a few. As stated by Admassu (2011) students are twice as likely to drop out of schools with such poor facilities.

In some places schoolgirls are very much affected by these poor quality facilities. For example, most of Nepal schoolgirls leave school early due to lack of sanitation facilities in schools (The Rising Nepal, access on 24/04/2012). The lack of proper toilets in schools in Kathmandu, Nepal, increases the dropout rate for schoolgirls from about $7 \%$ in 2001 to about $13 \%$ in 2007. In the same report, it has been shown that given the 148,000 toilets built annually, 59\% of public and community schools did not have one, and apart from that only $10 \%$ of the poor families have access to it. A similar finding by the World Bank (2005) unfolds how bad it is for African countries. As stated in their report, about 1 in 10 schoolgirls misses between $10 \%$ and $20 \%$ of her school days, due to the absence of clean and private sanitation facilities in schools. The academic achievement of these rural and urban schoolgirls hygienically depends 
on these facilities especially during menstruation (World Bank, 2005). They have also stated that female teachers too are affected by the lack of these physical facilities.

These findings express a concern about the lack of good quality physical facilities in schools as they contribute to school dropout rates (GNA, 2012). According to SAPPROS Nepal (2011), the classrooms for one of the primary schools in one of the districts, are considerably small with no doors and windows. Further, cattles very often used these classrooms as shelters especially during night time which made them dirty. As a result of these awkward situations the dropout rates for these schools could increase as high as about $50 \%$.

In the United States of America, Branham (2004) investigates the effects of these unstable school infrastructures on 226 independent schools in Texas. From his findings he clearly shows that for schools that use temporary buildings (about $5 \%$ of total school buildings square feet) have lower attendance rates (1 in 1000 student per day) compared to those that do not use temporary buildings. At the same time the number of dropouts excalates to about 10 per year. More interestingly, for schools that need structural repair have very low attendance rates (roughly 5 in 1000 students a day) but have higher dropout rates (at least 10 in 1000 students per year) than those that do not need structural repair. Similar results are shown by the Centre for Innovative School Facilities of Oregon (access 24/04/12) whereby schools with excellent facilities have higher achievement or success rates than schools with poor facilities. In addition, middle school students achieved better test scores in English, Maths and Science subjects in newer or recently renovated school buildings than in older ones.

In summing up based on these findings school buildings and facilities can also affect the students' academic performance as well as the dropout rates. More students will definitely drop out if the quality of school buildings and facilities are out of standards.

\subsection{Post-Dropout Drama and Dropout Prevention}

This study wishes to focus on what dropouts do after leaving school and what can be done to prevent it from happening; however the data that I am using does not have these information. Thus this subsection briefly summarises and identifies some of the activities that dropouts do or involve in after dropping out of school. It also talks about some initiatives to help reduce or prevent the school dropouts.

There is always a concern about victims of dropping out as some people fear that these early school leavers could be burdens to the community at large. From the literature, many of these dropouts are either twice as likely (Ream \& Rumberger, 
2008) or three times more likey (Malin \& Meidment, 2003; Lamb, 2011) to be unemployed. Similar findings are exposed by Rumberger (2001) and Meekers \& Ahmed (1999) where early school leavers are less likely to find a good job to earn enough for a living. Some of these dropouts are being employed by very low paying jobs to support their families (Naidu, Barr \& Seniloli, 2007) as compared to their counterparts who graduted with diplomas (Tyler \& Lofstrom, 2009). For example, dropouts can earn as much as 37 cents for every dollar earned by a high school graduate (Ream \& Rumberger, 2008). But according to Tyler \& Lofstrom (2009) female dropouts who are working only earn $65 \%$ of their counterparts' earning who graduated with diplomas, while male dropouts' earning is less than $70 \%$ of that of diploma holders of the same sex.

With the increasing number of these early school leavers not getting any job, the unemployment rates cost nations at large in terms of productivity and reduced tax income (Rumberger, 2001). For instance, Rumberger (2001) estimates that in one year dropouts contribute to about US $\$ 3.2$ billion in lost earnings and more than US $\$ 400$ million in social services. This is supported by Christenson \& Thurlow (2004) where they claim that dropouts cost societies about billion of dollars in lost revenue, welfare and unemployment programmes, as well as crime prevention and prosecution. Moreover, Tyler \& Lofstrom (2009) also argue in support of Rumberger's (2001) discoveries, that these early school leavers do not contribute much to tax revenue as they work in very low paying jobs. Not only that but they also have higher crime rates and increase public spending on public assistance and health care for them.

Many of the dropouts who are unable to get employments are most likely to be found on the streets (ESP, 2007) causing a lot of problems which find them behind bars. For instance, Tyler \& Lofstrom (2009) unfold that many dropouts in the United States are overrepresented in the prisons and they make up 68\% of the nation's inmates. These findings underpin Archwamety \& Katsiyannis (1998) discovery, where they unfold that a disproportionate number of female dropouts (about 82\%) are filling up correctional facilities in the States. The type of offence that these females committed includes armed robbery, gang activity, drug dealings, burglary, weapons possession, aggrevated assault and prostitution (Archwamety \& Katsiyannis, 1998). The same author(s) report that females are more likely to reoffend over and over again especially those from urban areas. They also claim that dropouts are about 4 times more likely to be arrested than graduates.

However, not all dropouts are troublemakers, some may decide to go back to school after a while, or choose alternative education such as Technical and Vocational Education and Training (TVET) (Lamb, 2011) to continue their education. Alternative pathways such as TVET may help reduce both the dropout rates and the unemployment rates. For example, Lamb (2011) finds that dropouts from low socioeconomic backgrounds and rural areas as well as disable students, re-engage with education to improve their achievement level by making use great opportunities provided by TVET. 
The dropout problem can be in one way or another avoided or reduced if necessary actions and precautions are taken, as Benjamin Franklin says "An ounce of prevention is worth a pound of cure”. Free and compulsory education (ESP, 2007; Naidu, Barr \& Seniloli, 2007) law such as liangmian yibu (Yi, et.al, 2011) must be in place and enforce them if we really want to reduce the dropout rate and promote the completion rate (Christenson \& Thurlow, 2004). Yi, et.al (2011) insist that before the liangmian yibu law the dropout rate in China was as high as $8 \%$, but when the new law kicked in it dropped to less than $2 \%$ in the following year. Exit exams are believed to lower the completion rates and increase the dropout rates (Rumberger, 2001). Removing most of these exams might be helpful in reducing the dropout rate. For example, Fijilive (2009) has reported that the Minister of Education of Fiji officially announced the elimination of three of the five existing Fiji external exams in order to increase completion rates.

Offering Scholarship opportunities (Cameron, 2002; Cameron 2004) for at risk students especially those from lower socio-economic backgrounds, living in poor and below poverty level families (Naidu, Barr \& Seniloli, 2007), as well as schools with higher dropout rates (Cameron, 2004) is another option to consider in order to keep students in school. For instance, Cameron (2004) argues that scholarships have been very successful in reducing the dropout crisis at lower secondary schools in Indonesia by $38 \%$.

Intensive programs such as "Check \& Connect" (Tyler \& Lofstrom, 2009; Christenson \& Thurlow, 2004) for disengagement students, whereby parents, families, community services organisations, schools and the education community all work together to support and assist their students in one way or another, for them to stay in and successfully complete their education. Similar intervention programs have also mentioned by Pittman (1991) and Vosamana (2012). Another model called "Project Head Start" (Malin \& Meidment, 2003) which is quite similar to "Check \& Connect", whereby teachers play a supportive role rather than directive in facilitating children's initiative and social relations development, creativity, music and other activities, is another recommended approach in lowering the dropout rates.

Students' participation in extra-curricular activities (Ream \& Rumberger, 2008; Pittman, 1991; ESP, 2007; McNeal, 1995) such as sports and fine arts, other than academic curriculum may also help prevent the dropout fever. Non-formal trainings and programs for both affected students and parents (Veramu, 2002; Naidu, Barr \& Seniloli, 2007) coupled it with vocational education such as TVET (Veramu, 2002; ESP, 2007; Lamb, 2011) could also downgrade the dropout rates.

Other researchers argue that "school-within-school" models and "Career Academy" (Tyler \& Lofstrom, 2009) may also do the job in shrinking the dropout problem. The school-within-school approach is where a small group of students or class, especially at risk students learning from the same teacher for at least 3 consecutive years of high school. This is kind of similar to reducing the class size for better learning environment (Christenson \& Thurlow, 2009). The "Career Academy" refers to the 
combination of the "school-within-school" model, the academic and vocational curriculum, and the partnership between the academy and employers, to provide career and work-based learning opportunities for disadvantaged students in particular.

Constant counselling (Christenson \& Thurlow, 2004; Veramu, 2002) especially at risk students as well as financial aids for rural schools (Naidu, Barr \& Seniloli, 2007), should also be considered if we really care for victims of dropping out. Veramu (2002) on the other hand, points out that policymakers of education and school operations should also review existing education or school policies and practices such as, increasing the upper limit of the compulsory age group (Tyler \& Lofstrom, 2009) as an alternative to keep students in school much longer.

Another approach that may also help reduce the dropout rate is called the "Positive Peer Culture" (PPC) (Archwamety \& Katsiyannis, 1998), where positive social influence of peers of at risk students on these at risk students. It is an approach commonly used by correctional facilities in the States to help misconduct dropouts who are being incarcerated.

Improving and upgrading the quality of school buildings, school facilities and resources as well as teachers is most importantly mattered in reducing the dropout disaster. Branham (2004) argues that the quality of school building also has an impact on dropout rates. Upgrading these facilities will definitely make a change as SAPPROS Nepal (2011) reports that after reconstruction of new school buildings in the affected school in Nepal, the dropout rates dropped to $17 \%$ from $49 \%$.

Last but not least Tyler \& Lofstrom (2009) recommends assigning of unique identification numbers (or student education numbers) to each student in a way to provide accurate information and statistics on enrolment and completion as well as dropouts and transfers.

\subsection{Conclusion}

From the literature, the dropout problem spreads worldwide left, right and centre. It appears that students from poor, low socio-economic, below poverty line and ethnicity minority backgrounds and families are mostly affected, especially those from rural areas. Nevertheless, students from unstable family structures such as family mobility for instance, are also affected by the dropout symptoms. There are many factors and reasons mentioned as why most of the students have left schools early. Such factors include students' characteristics, peers influences, health problems like pregnancy, socio-economic status and most of all family and school factors such 
as teachers, buildings, resources and facilities. These factors really disadvantaged public school students especially older ones or those attending high schools.

Despite government reforms, education and school policies to reduce the dropout rate, yet some regions and schools are still facing and fighting with higher dropout rates. To some extent, some members of the dropout clan end up in prisons while some struggle to find employments. Fortunately, fewer from these groups of early school leavers get to enjoy their small earnings from their low paying jobs, but for others they will have to try their luck again. However, school dropouts create much chaos for societies financially as many are unemployed.

From the literature review we know that some of the preventions and intervention programs may or may not work for Samoan school dropouts as Christenson \& Thurlow (2004) argue that: "it is unlikely that a program developed elsewhere can be duplicated exactly in another site, because local talents and priorities for school reform, the particular interests and needs of the students to be served, and the conditions of the school to be changed will differ" ( $\mathrm{pp} 256)$. 


\section{Chapter 3}

\section{The Dropout Rate Overview}

This chapter focuses on the overview of the dropout rates in Samoan schools from 1995 to 2006. We only use the 1995 to 2006 because the enrolment data for both Primary and Secondary schools were provided for these years. We have excluded the 2007 year from this overview due to the unavailability of enrolment data for this year. We will first look at the definition of the dropout rate used by Samoa's Ministry of Education, Sports and Culture in section 3.1. Then the dropout rate status by region in section 3.2, followed by the dropout rate status by school level in section 3.3 and the dropout rate status by school status in section 3.4. The dropout rate status by region, school level and school status is measured and compared by the median dropout rates by Year level (as in Yr 2). These median dropout rates as in Table 3.1, Table 3.2 and Table 3.3 were calculated from the data provided by all existing schools (Primary and Secondary) in 1995 up until 2006. There were 192 schools in 1995, 193 in 1996, 196 in 1997 and 2004, 199 in 1998 and 2005, 198 in 1999 and 2000, 202 in 2001, 203 in 2002, 201 in 2003 and 200 in 2006.

\subsection{Definition}

The perception of the Samoan community towards students who are dropping out of schools at some point varies from case to case due to reasons associated with the students not being in school. However, generally speaking when a student stops attending school no mater what level, for more than at least a week he/she is regarded as a dropout. But according to the Ministry of Education, Sports and Culture (MESC), the definition of dropout is expressed as a rate and it does not account for students who are being migrated overseas or transferred from one school to another. Ideally, the study wishes to get the data that can track individual students but it is not the case for the survey data that we are using for this study. Thus the analysis we did was restricted to the dropout rate in the given survey data. 
Dropout Rate: The percentage of students who neither progress from one year level to the next nor repeat the year level. (Ministry of Education, Sports and Culture, 2007)

The calculation of the dropout rate is based on the formula developed by the UNESCO where both the promotion and repetition rates are subtracted from 100 to get the dropout rate. That is, $\mathrm{DR}=100-\mathrm{PR}-\mathrm{RR}$, where $\mathrm{DR}$ is the dropout rate, $\mathrm{PR}$ is the promotion rate and $\mathrm{RR}$ is the repetition rate. This is the recommended formula to use across the region, as most of the school administrative data do not gather information on dropouts.

The promotion rate (PR) is defined as the proportion of students who have successfully completed a school year level (grade) and proceeded to the next school year level (grade) the following year. That is, the promotion rate of school year level (grade) ' $\mathrm{g}$ ' is the number of promotes at school year level (grade) ' $\mathrm{g}+1$ ' in calendar year ' $t+1$ ', divided by the number of students enrolled in school year level (grade) ' $\mathrm{g}$ ' in calendar year ' $\mathrm{t}$ ' and multiplied the results by 100 . For the repetition rate (RR), it is the proportion of students who repeat a school year level (grade). This is calculated by dividing the number of students who repeat school year level ' $\mathrm{g}$ ' in calendar year ' $t+1$ ' by the number of students who enrolled in school year level ' $\mathrm{g}$ ' in calendar year ' $t$ ' and multiplying the result by 100 .

For this study, negative dropout rates have been changed to zeros. This is based on the understanding that dropout rates should be between zero and 100. Negative dropout rates are caused by new students being enrolled in the school in a particular year level, who have been either transferred from one school to another, migrated from overseas or even skipping year levels within the same school year. Transferred students are not being captured by the MESC Census due to the absence of questions in the Census Form (see Appendix D) to address these transferred students.

\subsection{Dropout Rate Status by Region}

From 1995 to 2006, we first calculated the median before we look at the trend across all these calendar years. For all these calendar years, higher median dropout rates for Year 2, Year 7, Year 10, Year 12 and Year 13 levels were found in the Rest of Upolu region as compared to those of the Apia Urban and Savaii regions. For Year 11 level, the Apia Urban has the highest as shown in Table 3.1. In some school year levels across all the three regions, the dropout rate is similar. The table shows that the dropout rate for Savaii region is higher in Year 2, Year 10, Year 12 and Year 13 than those of the Apia Urban region. However, comparing the median dropout rates for all three regions, it seems like students were more likely to drop out of schools in the rural areas (Rest of Upolu and Savaii regions) than in the urban area (Apia Urban). This is graphically supported by Figure 3.1 to Figure 3.6. Nevertheless, the dropout 
rate across all the three school regions has been improved over the years which is very apparent in some school year levels as shown by Figures 3.1 to 3.6 except for Figure 3.4. Furthermore, the dropout rate is kind of stable for most school year levels except for Year 13 as displayed by Figure 3.6.

Table 3.1: School Year Level Median Dropout Rates of all schools by Region

\begin{tabular}{|c|c|c|c|c|c|c|c|c|c|c|c|c|c|}
\hline \multirow{2}{*}{ Region } & & \multicolumn{12}{|c|}{ School Year Level } \\
\hline & & $\mathrm{Yr} 2$ & $\mathrm{Yr}_{\mathrm{r}} 3$ & Yr 4 & Yr 5 & $\operatorname{Yr} 6$ & $\operatorname{Yr} 7$ & Yr 8 & Yrg & $\operatorname{Yr} 10$ & Yr 11 & $\mathrm{Yr} 12$ & Yr 13 \\
\hline $\begin{array}{l}\text { Apia } \\
\text { Urban }\end{array}$ & $\begin{array}{l}\text { Median } \\
(Q 1, Q 3)\end{array}$ & $\begin{array}{c}2.39 \\
(0,16.67)\end{array}$ & $\begin{array}{c}0 \\
(0,9.52)\end{array}$ & $\begin{array}{c}0 \\
(0,7,14)\end{array}$ & $\begin{array}{c}0 \\
(0,9.71)\end{array}$ & $\begin{array}{c}0 \\
(0,9.09)\end{array}$ & $\begin{array}{c}1.82 \\
(0,11.76)\end{array}$ & $\begin{array}{c}0 \\
(0,8)\end{array}$ & $\begin{array}{c}0 \\
(0,0)\end{array}$ & $\begin{array}{c}4.92 \\
(0,10.57)\end{array}$ & $\begin{array}{c}7.52 \\
(0,23.24)\end{array}$ & $\begin{array}{c}0 \\
(0,2.56)\end{array}$ & $\begin{array}{c}6.32 \\
(0,24)\end{array}$ \\
\hline $\begin{array}{l}\text { Rest of } \\
\text { Upolu }\end{array}$ & \begin{tabular}{|l|} 
Median \\
(Q1, Q3)
\end{tabular} & $\begin{array}{c}7.14 \\
(0,22.22)\end{array}$ & $\begin{array}{c}0 \\
(0,11.11)\end{array}$ & $\begin{array}{c}0 \\
(0,9.09)\end{array}$ & $\begin{array}{c}0 \\
(0,12.5)\end{array}$ & $\begin{array}{c}0 \\
(0,11111)\end{array}$ & $\begin{array}{c}3.33 \\
(0,13.33)\end{array}$ & $\begin{array}{c}0 \\
(0,10.87)\end{array}$ & $\begin{array}{c}0 \\
(0,25)\end{array}$ & $\begin{array}{c}11.32 \\
(3.08,19.09)\end{array}$ & $\begin{array}{c}5.71 \\
(0,18.87)\end{array}$ & $\begin{array}{c}17.39 \\
(0,37.74)\end{array}$ & $\begin{array}{c}34.15 \\
(177.39,56.67)\end{array}$ \\
\hline Savaii & $\begin{array}{l}\text { Median } \\
(\text { Q1, Q3) }\end{array}$ & $\begin{array}{c}6.21 \\
(0,20)\end{array}$ & $\begin{array}{c}0 \\
(0,8.16)\end{array}$ & $\begin{array}{c}0 \\
(0,8.33)\end{array}$ & $\begin{array}{c}0 \\
(0,9.09)\end{array}$ & $\begin{array}{c}0 \\
(0,9.52)\end{array}$ & $\begin{array}{c}0 \\
(0,10)\end{array}$ & $\begin{array}{c}0 \\
(0,0)\end{array}$ & $\begin{array}{c}0 \\
(0,0)\end{array}$ & $\begin{array}{c}8.16 \\
(0,16.67)\end{array}$ & $\begin{array}{c}0 \\
(0,11.84)\end{array}$ & $\begin{array}{c}3.95 \\
(0,32.84)\end{array}$ & $\begin{array}{c}20.93 \\
(0,53.97)\end{array}$ \\
\hline
\end{tabular}

Note: For each cell, the number at the top is the median and the numbers at the bottom inside the brackets are the Lower Quartile (Q1) and the Upper Quartile (Q3), for all calendar years (from 1995 to 2006).

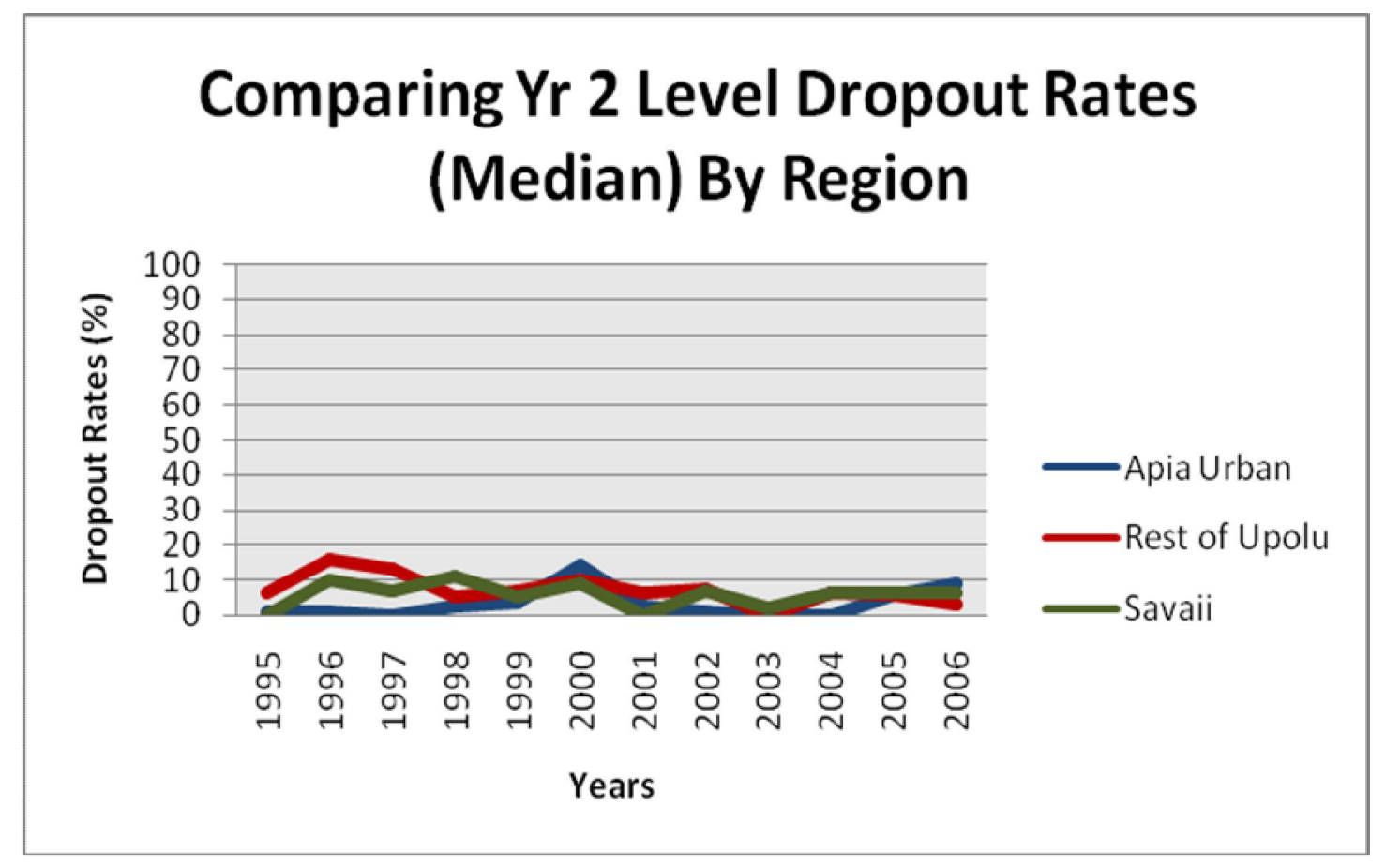

Figure 3.1: Comparing Year 2 Level dropout Rates by Region, 1995 - 2006 


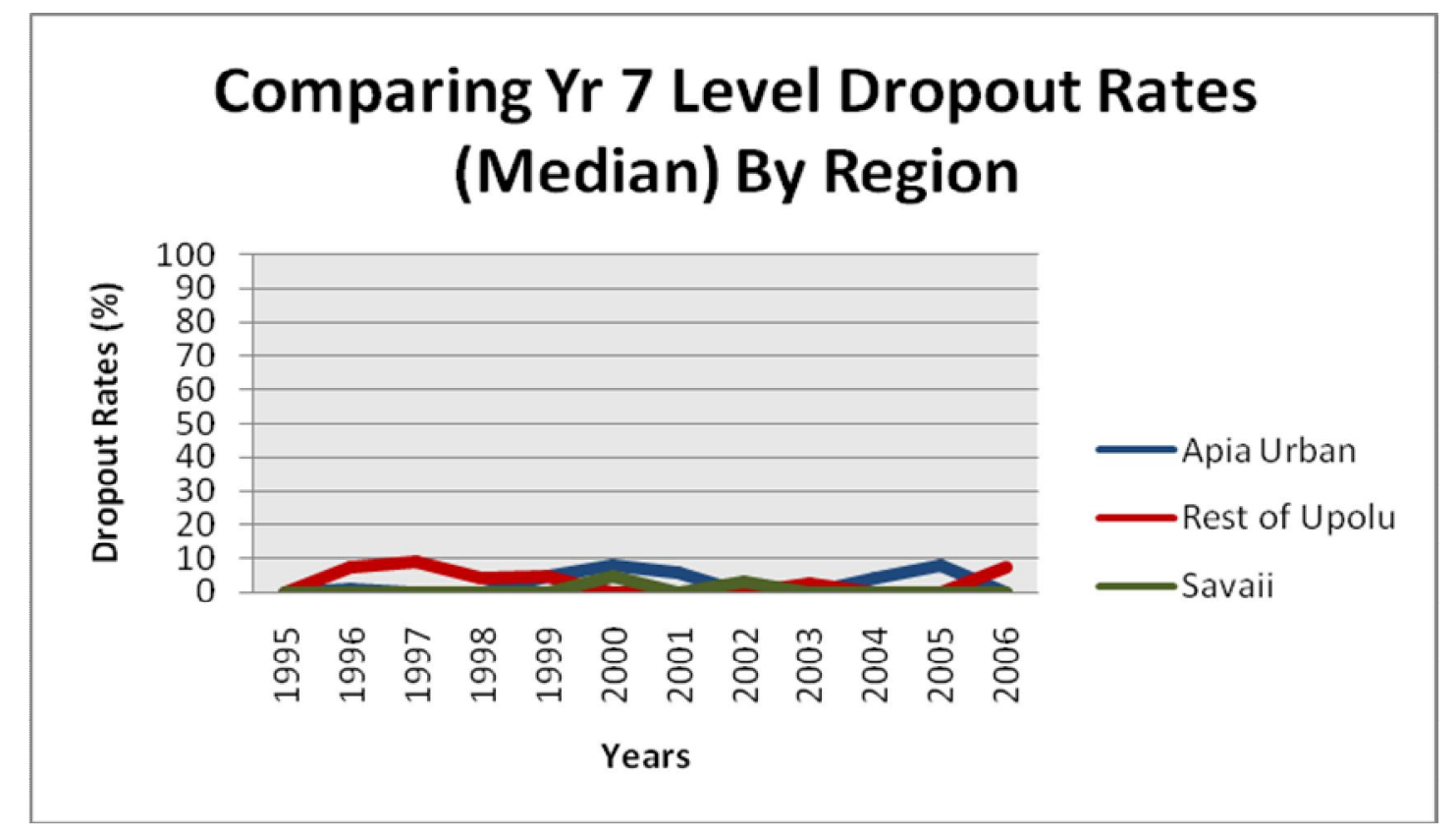

Figure 3.2: Comparing Year 7 Level Dropout Rates by Region, 1995 - 2006

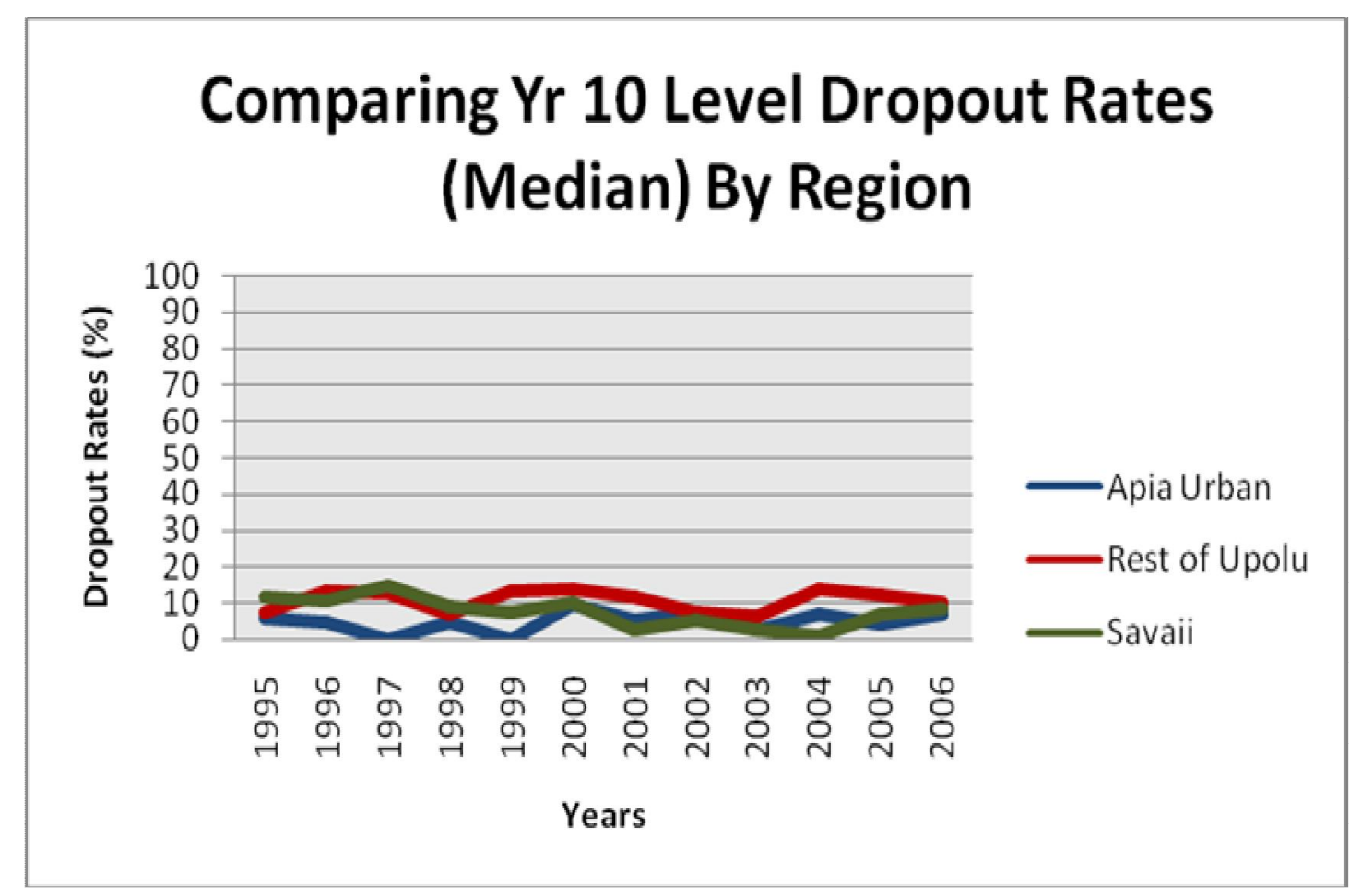

Figure 3.3: Comparing Year 10 Level Dropout Rates by Region, 1995 - 2006 


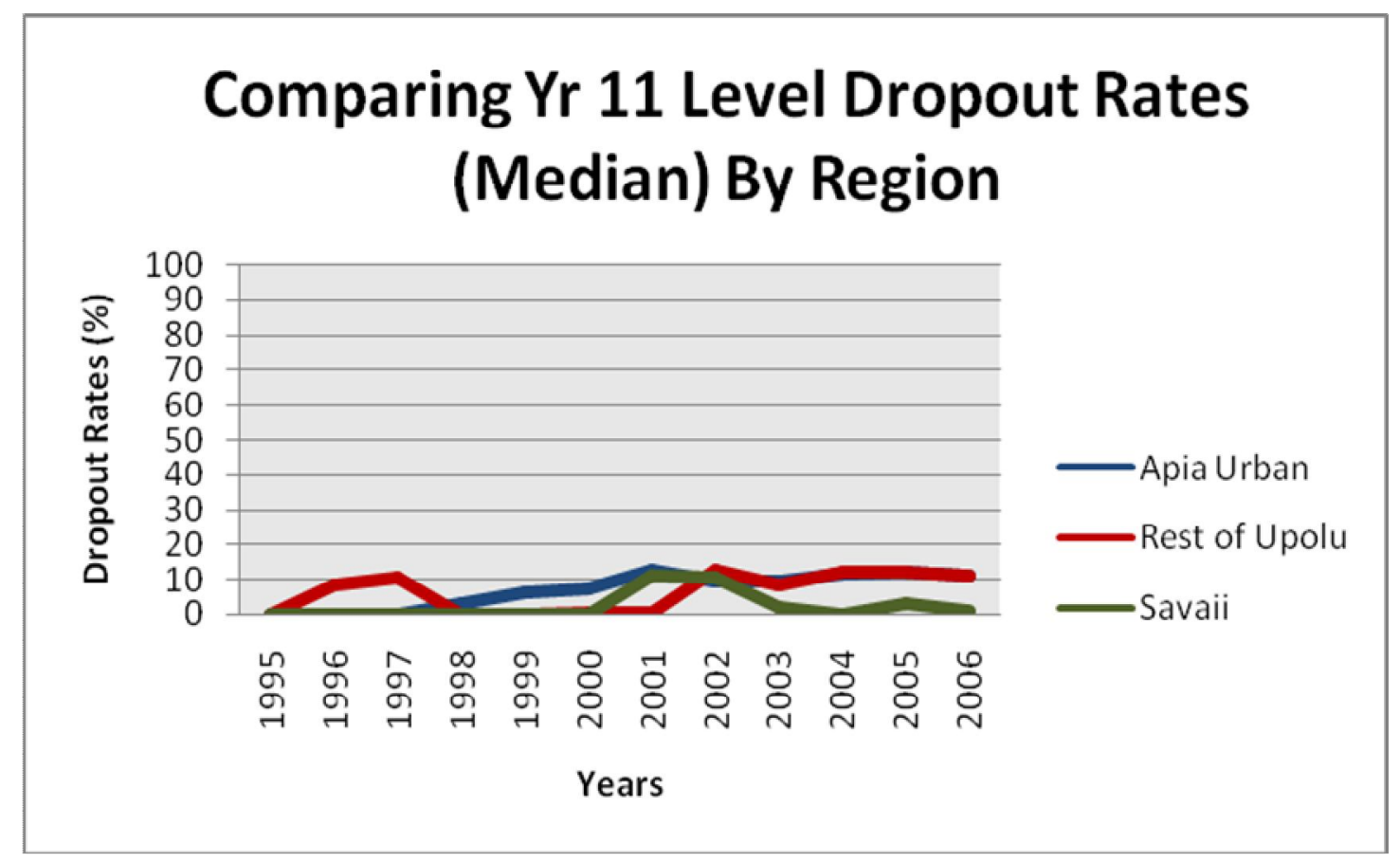

Figure 3.4: Comparing Year 11 Level Dropout Rates by Region, 1995 - 2006

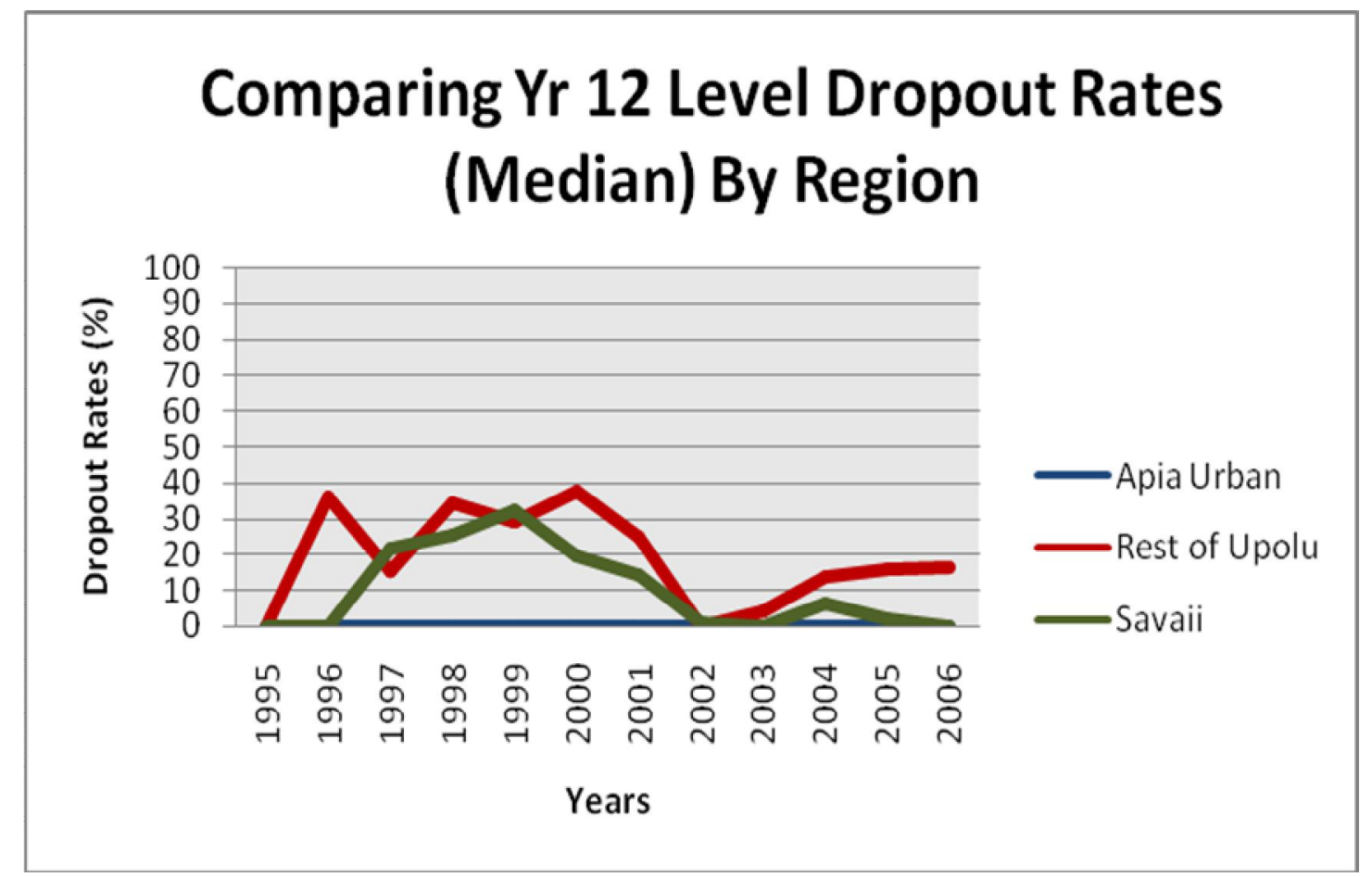

Figure 3.5: Comparing Year 12 Level Dropout Rates by Region, 1995 - 2006

Note: The sharp peaks are due to either one or two observations (schools) with higher dropout rates. 


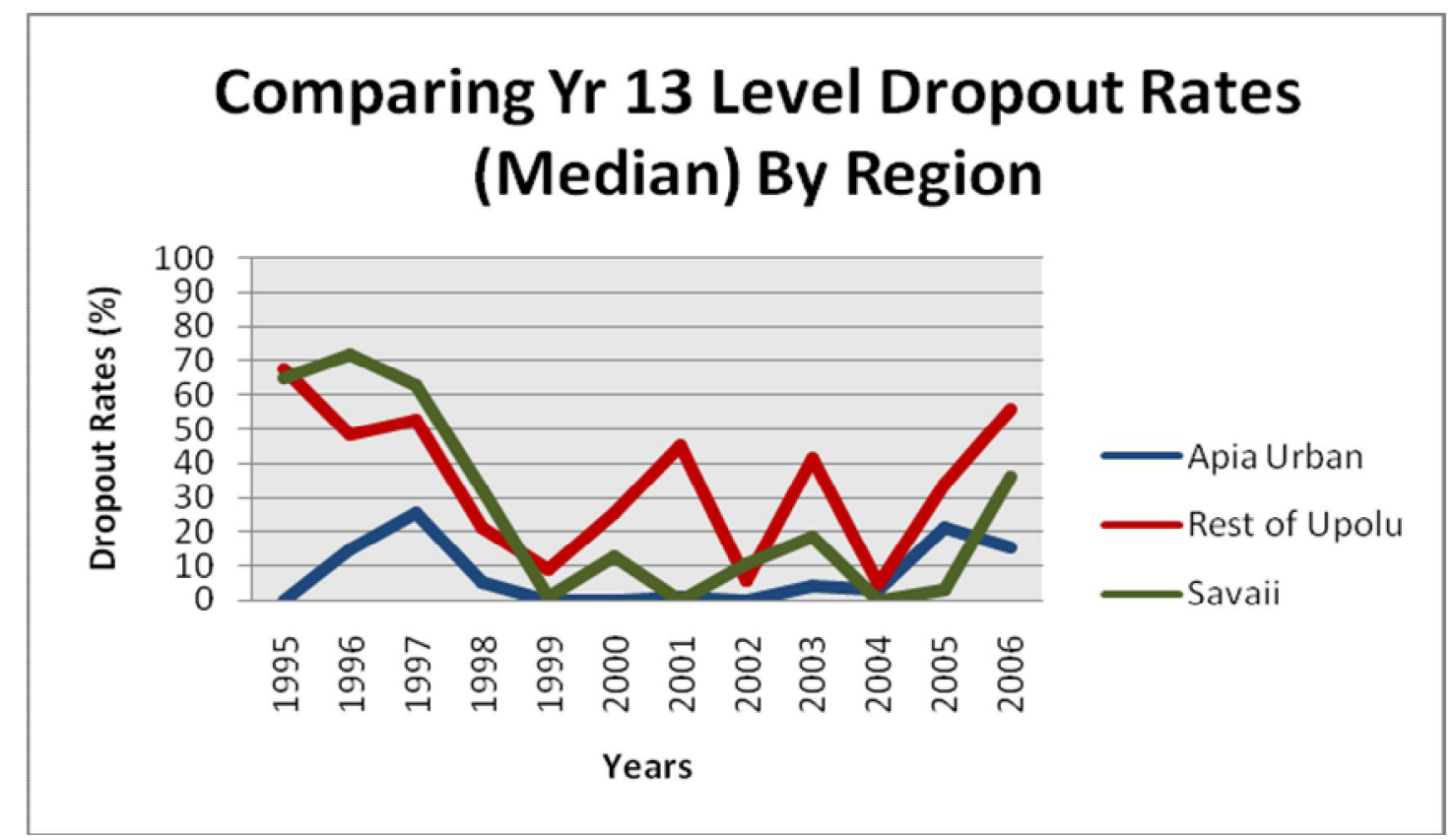

Figure 3.6: Comparing Year 13 Level Dropout Rates by Region, 1995 - 2006

Note: The sharp peaks are due to either one or two observations (schools) with higher dropout rates.

Figure 3.6 shows some large dropout rate for some calendar years especially in the Rest of Upolu and Savaii regions. In 1995 to 1999 the median dropout rates for the Rest of Upolu region are 67.29, 48.32, 52.78, 21 and 8.70 respectively. These extreme values were based on few schools. For instance, from 1995 to 1997 there was only one school in each calendar year and from 1998 to 1999 there were only two schools in each calendar year. As for the Savaii region, the same dropout pattern as in the rest of Upolu region was also seen from 1995 to 1999 (figures based on the range of three to six observations). In 2000, 2002 and 2003 figures were based on six (6 schools) and four (4 schools) observations respectively but not as high as the Rest of Upolu. More students were somehow leaving the schools again in 2005 and 2006 considerably across all the three school regions.

\subsection{Dropout Rate Status by School Level}

Table 3.2 shows that higher dropout rates were observed in Year 2, Year 3, Year 5 and Year 7 of Primary/Secondary schools compare to those of the respective school year levels of the Primary schools. However, higher dropout rates were detected at schools in the Secondary level category than schools in the Primary/Secondary level at all school year level (Yr 9 - Yr 13) except for Year 11. There was no dropout rate for Year 9 in the Secondary level as this is the first year level at Secondary schools in Samoa. 
Table 3.2: School Year Level Median Dropout Rates of all schools by School Level

\begin{tabular}{|c|c|c|c|c|c|c|c|c|c|c|c|c|c|}
\hline & & \multicolumn{12}{|c|}{ School Year Level } \\
\hline $\begin{array}{l}\text { School } \\
\text { Level }\end{array}$ & & Yr 2 & Yr 3 & $\operatorname{Yr} 4$ & $\operatorname{Yr} 5$ & $\operatorname{Yr} 6$ & $\operatorname{Yr} 7$ & Yr 8 & $\operatorname{Yr} 9$ & Yr 10 & Yr 11 & Yr 12 & Yr 13 \\
\hline Primary & $\begin{array}{l}\text { Median } \\
(Q 1, Q 3)\end{array}$ & $\begin{array}{c}5.88 \\
(0,20.83)\end{array}$ & $\begin{array}{c}0 \\
(0,9.8)\end{array}$ & $\begin{array}{c}0 \\
(0,8.33)\end{array}$ & $\begin{array}{c}0 \\
(0,10.71)\end{array}$ & $\begin{array}{c}0 \\
(0,10)\end{array}$ & $\begin{array}{c}0 \\
(0,11.76)\end{array}$ & $\begin{array}{c}0 \\
(0,7.69)\end{array}$ & & & & & \\
\hline $\begin{array}{l}\text { Primaryl } \\
\text { Secondary }\end{array}$ & $\begin{array}{l}\text { Median } \\
(Q 1, Q 3)\end{array}$ & $\begin{array}{c}26.04 \\
(0,45)\end{array}$ & $\begin{array}{c}8.39 \\
(0,23.08)\end{array}$ & $\begin{array}{c}0 \\
(0,16.67)\end{array}$ & $\begin{array}{c}8.13 \\
(0,22.2)\end{array}$ & $\begin{array}{c}0 \\
(0,0)\end{array}$ & $\begin{array}{c}12.18 \\
(0,37.5)\end{array}$ & $\begin{array}{c}0 \\
(0,14.22)\end{array}$ & $\begin{array}{c}0 \\
(0,0)\end{array}$ & $\begin{array}{c}6.32 \\
(0,20.51)\end{array}$ & $\begin{array}{c}4.48 \\
(0,13.21)\end{array}$ & $\begin{array}{c}0 \\
(0,2.71)\end{array}$ & $\begin{array}{c}0 \\
(0,4.88)\end{array}$ \\
\hline Secondary & $\begin{array}{l}\text { Median } \\
(\text { Q1, Q3) }\end{array}$ & & & & & & & & & $\begin{array}{c}7.83 \\
(0,16.67)\end{array}$ & $\begin{array}{c}3.33 \\
(0,17.86)\end{array}$ & $\begin{array}{c}2.49 \\
(0,31.55)\end{array}$ & $\begin{array}{c}20.25 \\
(0,48.86)\end{array}$ \\
\hline
\end{tabular}

Note: For each cell, the number at the top is the median and the numbers at the bottom inside the brackets are the Lower Quartile (Q1) and the Upper Quartile (Q3), for all calendar years (from 1995 to 2006).

In Figure 3.7, the dropout rate status at the Primary school level appears to have been improved over the years between 1995 and 2006 for most of the school year levels except for Year 2 and Year 7 in particular. The Year 2 level dropout rate has gradually improved but gone up again in 2004 to 2006 but still considerably and consistently higher than all the other year levels in the Primary school category. There were some considerable shifts in the dropout rates for Year 7 in 1996 and 1999 but then gradually dropped in the following years until 2006 where it slightly gone up again.

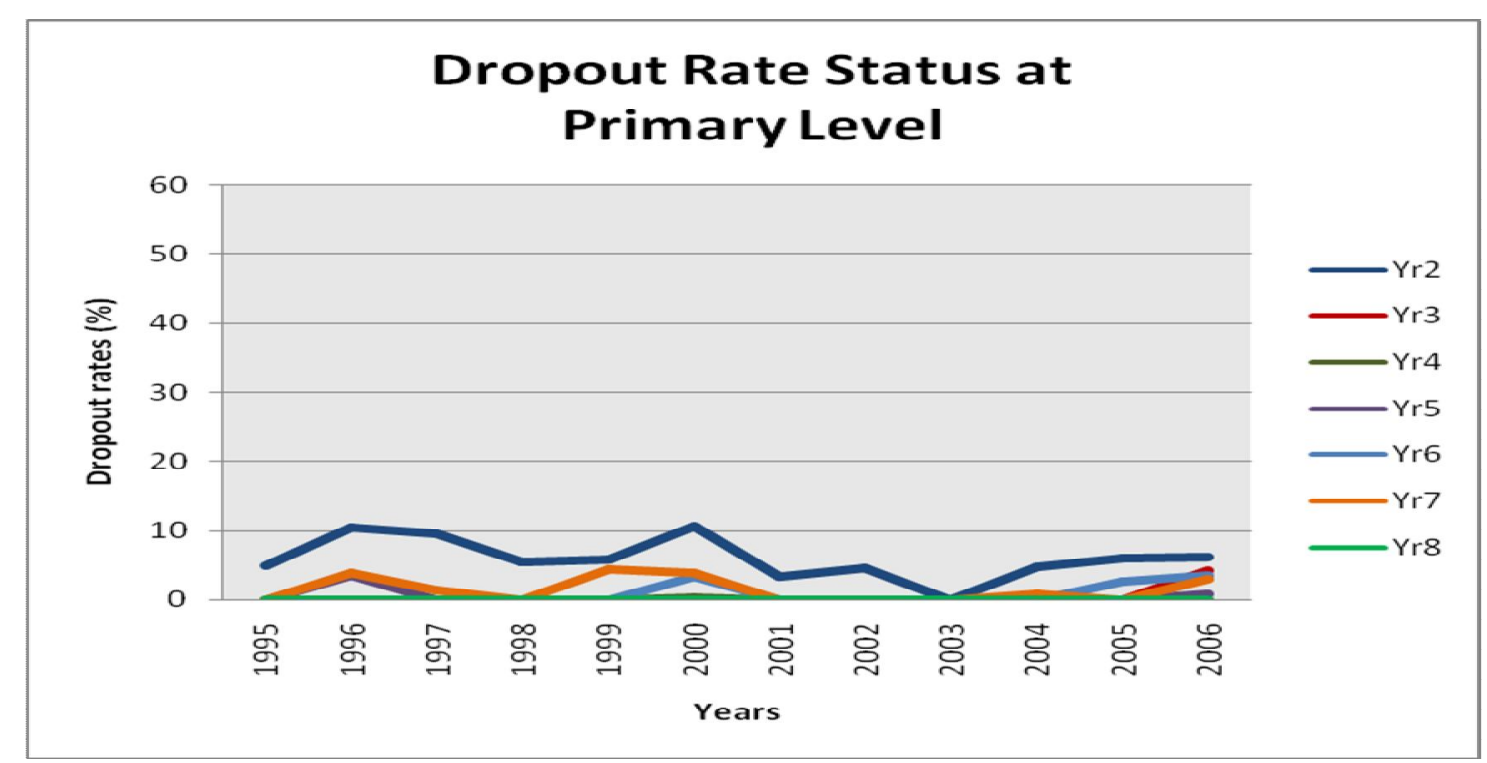

Figure 3.7: Dropout Rate Status at Primary Level, 1995 - 2006 
At Secondary school level, the dropout rate was also improved over the years (1995 2006) for all of the school year levels except for Year 13, where it continuously gone up again in 2005 and 2006 as shown by Figure 3.8. From 1995 to 2000, the dropout crisis for all the school year levels except for Year 11 was terrible in contrast to 2001 until 2006. It was the reverse for Year 11 during these years.

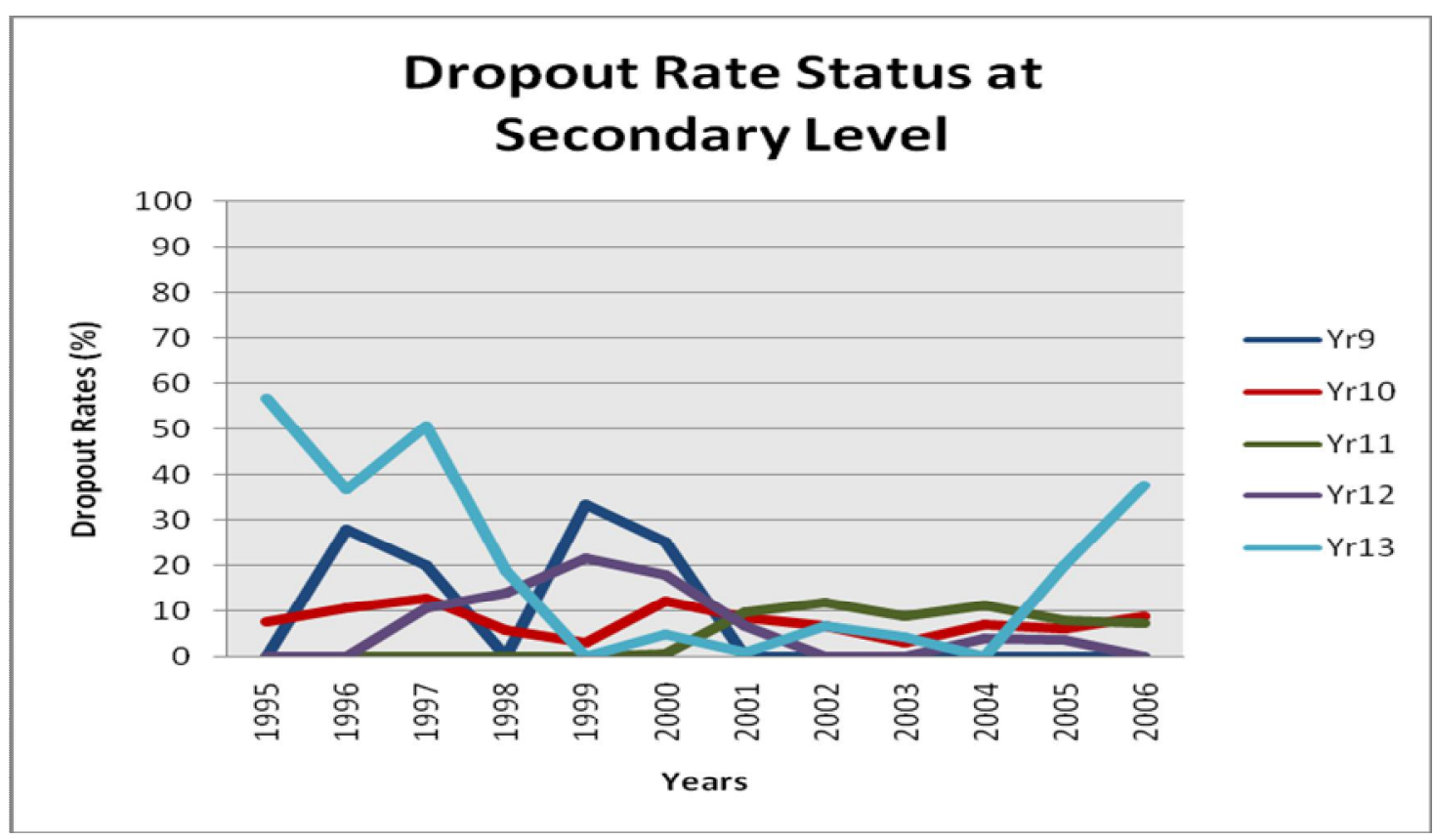

Figure 3.8: Dropout Rate Status at Secondary Level, 1995 - 2006

\subsection{Dropout Rate Status by School Status}

When comparing the dropout rates between the three school status (Government, Mission and Private), the Government schools appear to have higher dropout rates than both Mission and Private schools, particularly in Year 2, Year 9, Year 10, Year 11 and Year 12. The Mission schools have higher dropout rates in Year 13 than Government and Private schools as shown by Table 3.3. The Private schools have higher dropout rates in Year 6 and Year 7 compare to those of both Government and Mission schools of the same school year levels. Overall, during 1995 until 2006 many students were more likely to drop out of Government schools than in Mission and Private schools. This is evidently shown by Figure 3.9 to Figure 3.14 .

There are some missing dropout rates for both Year 11 and Year 12 school year levels for the Private schools category in Table 3.3. This is due to the fact that there are only two Private secondary schools exist in Samoa and both of them do not have Year 11 but do have Year 12 school year level. Students who successfully complete Year 10 automatically proceed to Year 12 the following year. 
Table 3.3: School Year Level Median Dropout Rates of all schools by School Status

\begin{tabular}{|c|c|c|c|c|c|c|c|c|c|c|c|c|c|}
\hline \multirow{2}{*}{$\begin{array}{l}\text { School } \\
\text { Status }\end{array}$} & & \multicolumn{12}{|c|}{ School Year Level } \\
\hline & & Yr 2 & $\operatorname{Yr} 3$ & $\operatorname{Yr} 4$ & $\operatorname{Yr} 5$ & $\operatorname{Yr} 6$ & $\operatorname{Yr} 7$ & Yr 8 & $\operatorname{Yr} 9$ & Yr 10 & $\operatorname{Yr} 11$ & Yr 12 & Yr 13 \\
\hline Government & $\begin{array}{l}\text { Median } \\
(Q 1, Q 3)\end{array}$ & $\begin{array}{c}6.67 \\
(0,21.05)\end{array}$ & $\begin{array}{c}0 \\
(0,9.68)\end{array}$ & $\begin{array}{c}0 \\
(0,8.33)\end{array}$ & $\begin{array}{c}0 \\
(0,10.53)\end{array}$ & $\begin{array}{c}0 \\
(0,10)\end{array}$ & $\begin{array}{c}0 \\
(0,11.76)\end{array}$ & $\begin{array}{c}0 \\
(0,7,69)\end{array}$ & $\begin{array}{c}20 \\
(0,27.78)\end{array}$ & $\begin{array}{c}9.48 \\
(2.41,17.12)\end{array}$ & $\begin{array}{c}4.44 \\
(0,17.57)\end{array}$ & $\begin{array}{c}21.57 \\
(0,45.59)\end{array}$ & $\begin{array}{c}12.6 \\
(0,37.06)\end{array}$ \\
\hline Mission & $\begin{array}{l}\text { Median } \\
(Q 1, Q 3)\end{array}$ & $\begin{array}{c}2 \\
(0,17.19)\end{array}$ & $\begin{array}{c}0 \\
(0,10)\end{array}$ & $\begin{array}{c}0 \\
(0,10.64)\end{array}$ & $\begin{array}{c}0 \\
(0,15.05)\end{array}$ & $\begin{array}{c}0 \\
(0,8.54)\end{array}$ & $\begin{array}{c}0 \\
(0,12)\end{array}$ & $\begin{array}{c}0 \\
(0,8.82)\end{array}$ & $\begin{array}{c}0 \\
(0,0)\end{array}$ & $\begin{array}{c}4.1 \\
(0,14.63)\end{array}$ & $\begin{array}{c}2.7 \\
(0,17.86)\end{array}$ & $\begin{array}{c}0 \\
(0,0)\end{array}$ & $\begin{array}{l}18.66 \\
(0,50)\end{array}$ \\
\hline Private & $\begin{array}{l}\text { Median } \\
(Q 1, Q 3)\end{array}$ & $\begin{array}{c}0 \\
(0,13.17)\end{array}$ & $\begin{array}{c}0 \\
(0,12.25)\end{array}$ & $\begin{array}{c}0 \\
(0,13.66)\end{array}$ & $\begin{array}{c}0 \\
(0,11.23)\end{array}$ & $\begin{array}{c}4.23 \\
(0,14.29)\end{array}$ & $\begin{array}{c}2.56 \\
(0,20)\end{array}$ & $\begin{array}{c}0 \\
(0,25)\end{array}$ & $\begin{array}{c}0 \\
(0,0)\end{array}$ & $\begin{array}{c}6.07 \\
(0,12)\end{array}$ & & & $\begin{array}{c}0 \\
(0,0)\end{array}$ \\
\hline
\end{tabular}

Note: (i) For each cell, the number at the top is the median and the numbers at the bottom inside the brackets are the Lower Quartile (Q1) and the Upper Quartile (Q3), for all calendar years (from 1995 to 2006).

(ii) The two missing informations are due to only 2 Private schools and both do not have $\operatorname{Yr} 11$.

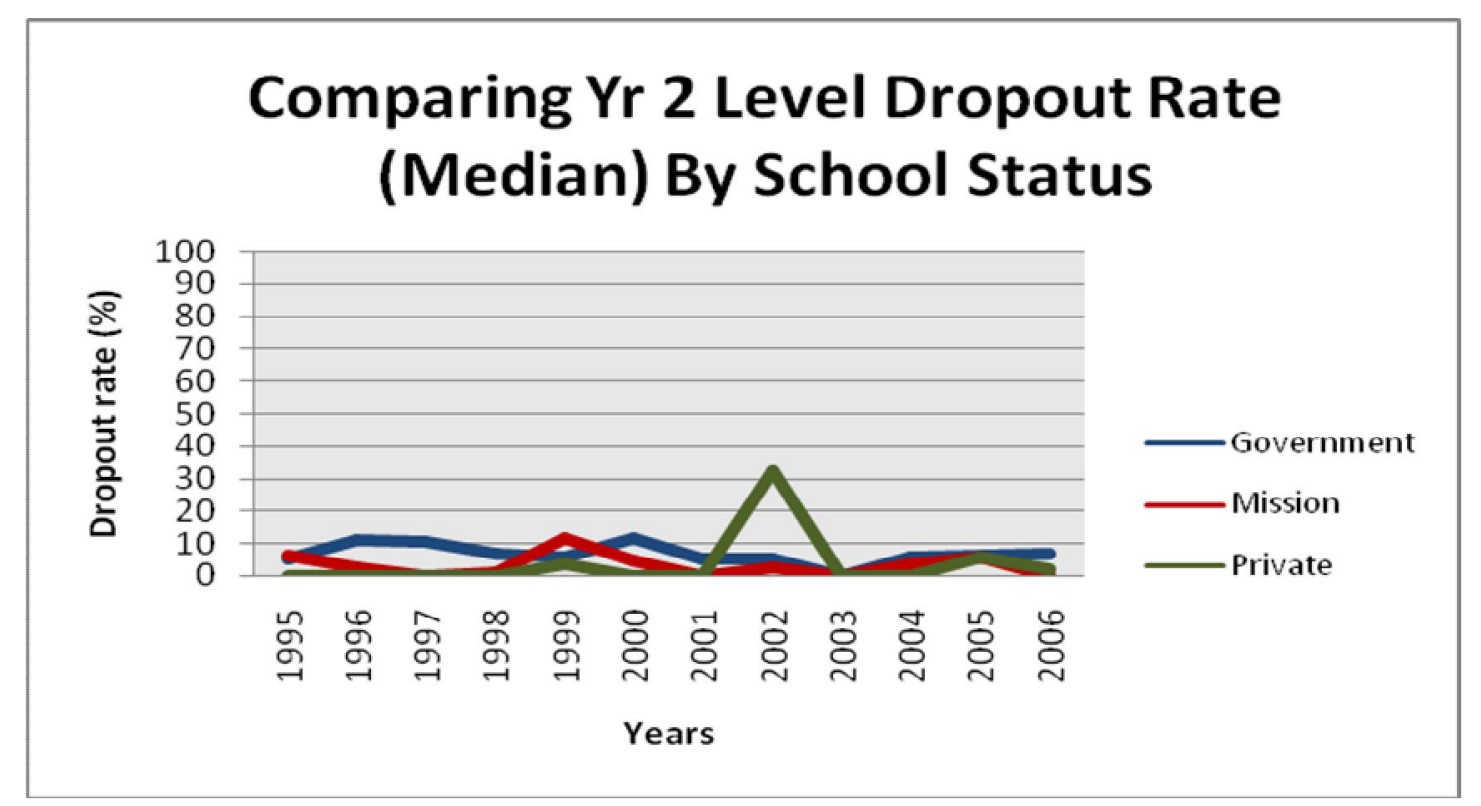

Figure 3.9: Comparing Year 2 Level Dropout Rates by School Status, 1995 2006

Note: The sharp peak is due to a single observation with a higher dropout rate.

Figure 3.9 shows that the dropout rate over the years across all three school status is improving for some calendar years for some school status. The dropout rate for 
Private schools is very high in 2002. This is because it is based on only one observation (1 school). Both Government and Mission schools, especially the Government, have higher dropout rates than the Private schools.

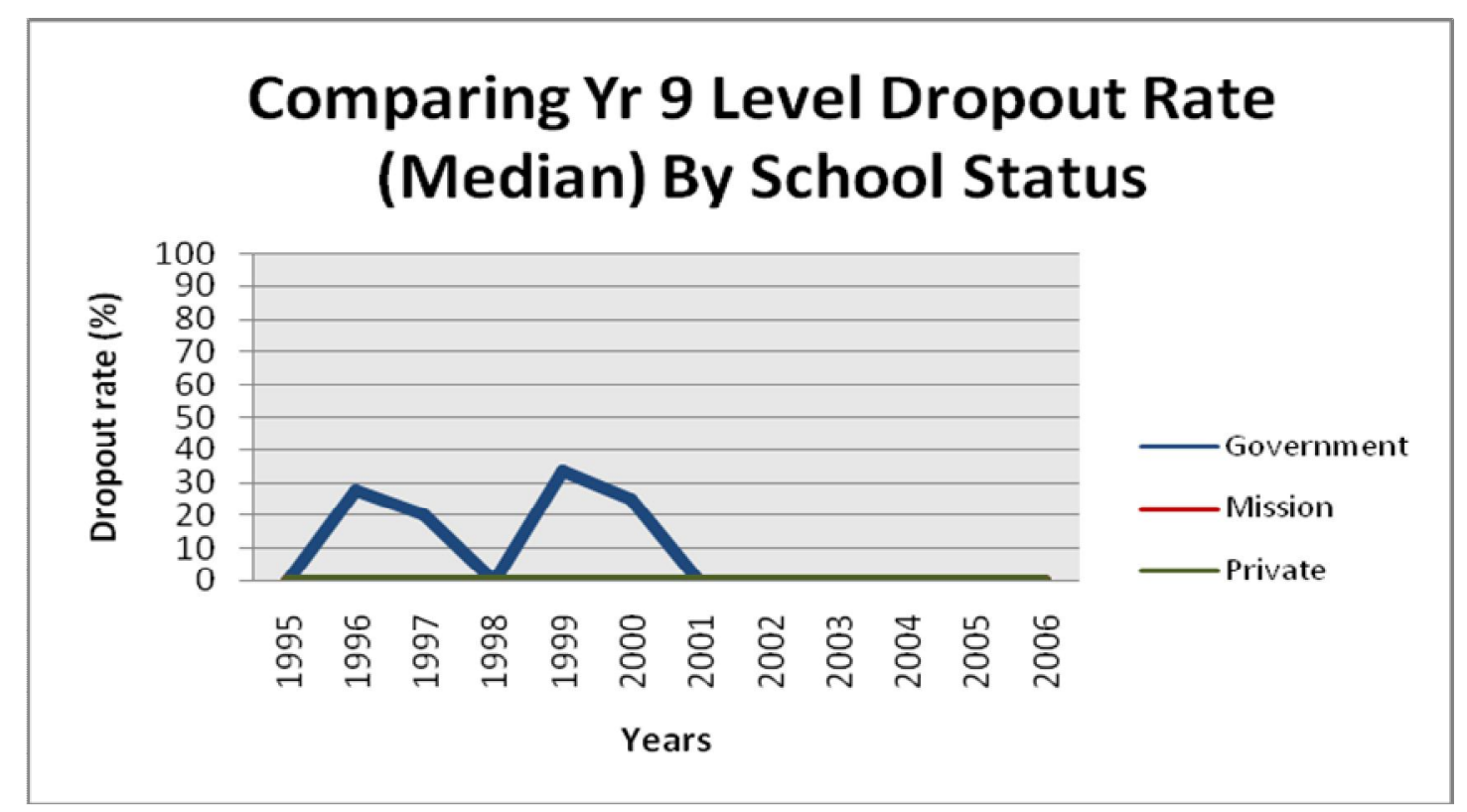

Figure 3.10: Comparing Year 9 Level Dropout Rates by School Status, 1995 2006

Note: The sharp peak is due to a single observation.

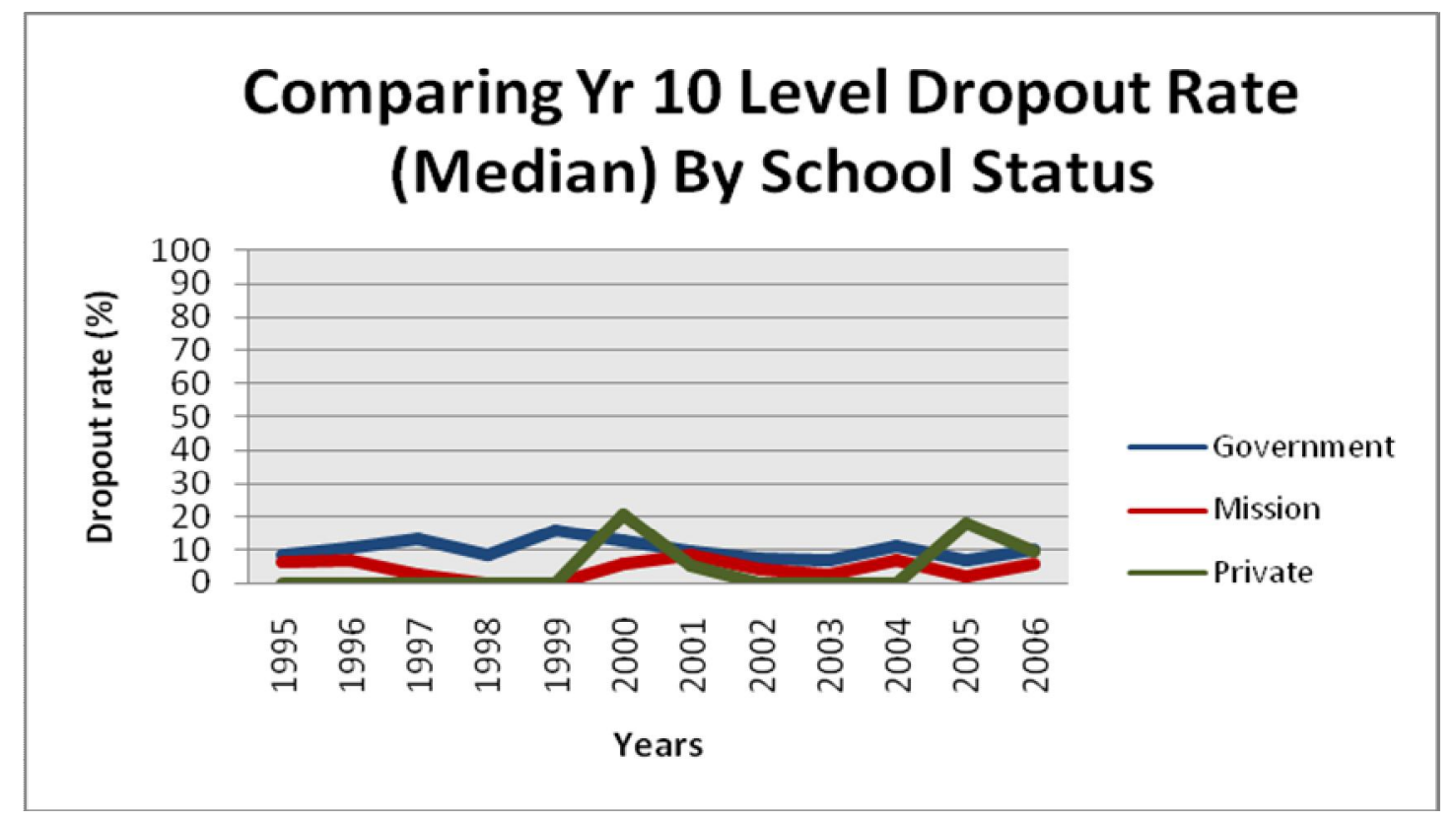

Figure 3.11: Comparing Year 10 Level Dropout Rates by School Status, 1995 2006

Note: The sharp peaks are due to either one or two observations. 
In Figure 3.10, higher dropout rates for Government schools were found in 1996, 1997, 1999 and 2000. All of these years the dropout rates were based on single observations (1 school only for each year). The Year 10 dropout rate as shown by Figure 3.11 has been improved over the years across all three school status, except for some years, significant changes have been found in the Private schools. Clearly, the dropout rates are higher in Government schools than in both Mission (even though dropout rates for Mission are higher than Private schools) and Private schools. The big changes for Private schools occurred in 2000 to 2001, and 2005 to 2006 whereby the dropout rates suddenly jumped up, because they were only based on single observations (in 2000 to 2001) and dual observations (in 2005 to 2006).

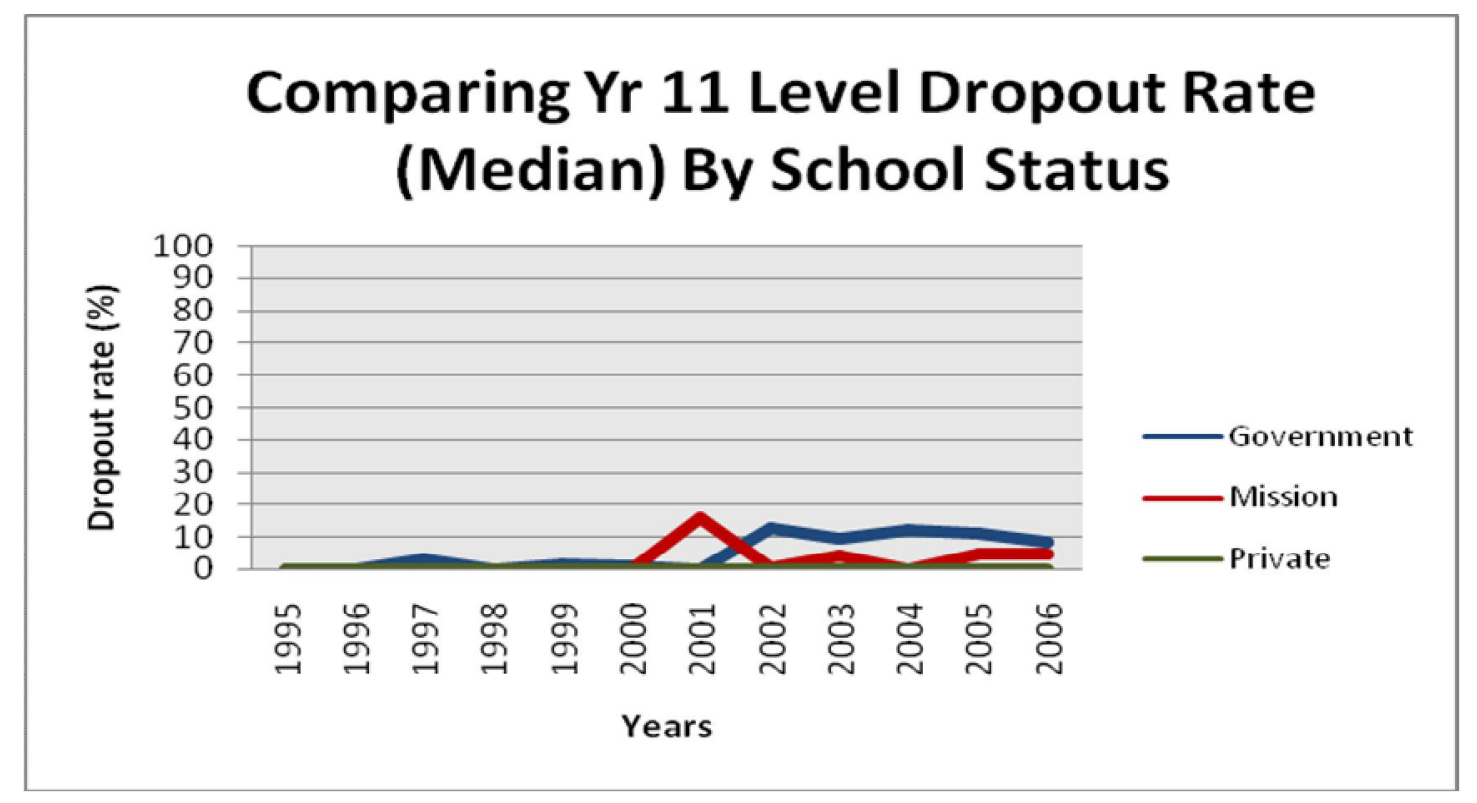

Figure 3.12: Comparing Year 11 Level Dropout Rates by School Status, 1995 2006

The Year 11 dropout rates seemed stable and very low from 1995 to 2001 for Government schools but suddenly ballooned to 12.73 in 2002 and constantly stabilised in the following years. From 2002 to 2006 dropout rates for Government schools were still much higher than any other school status in the country. Like the Government schools, as displayed by Figure 3.12, a much stable dropout rate condition was seen in 1995 to 2000 for Mission schools, but then there was this dropout rate hump found in 2001 before it became stabled again in the following years. Despite all these variations of the dropout rates in the beginning of the $21^{\text {st }}$ century, the fact still remains that more students are leaving schools in the Government status than in both the Mission and Private schools.

Figure 3.13 shows huge improvement on the dropout rates for Government schools in Year 12 but still remain high as opposed to those of Mission and Private schools. 


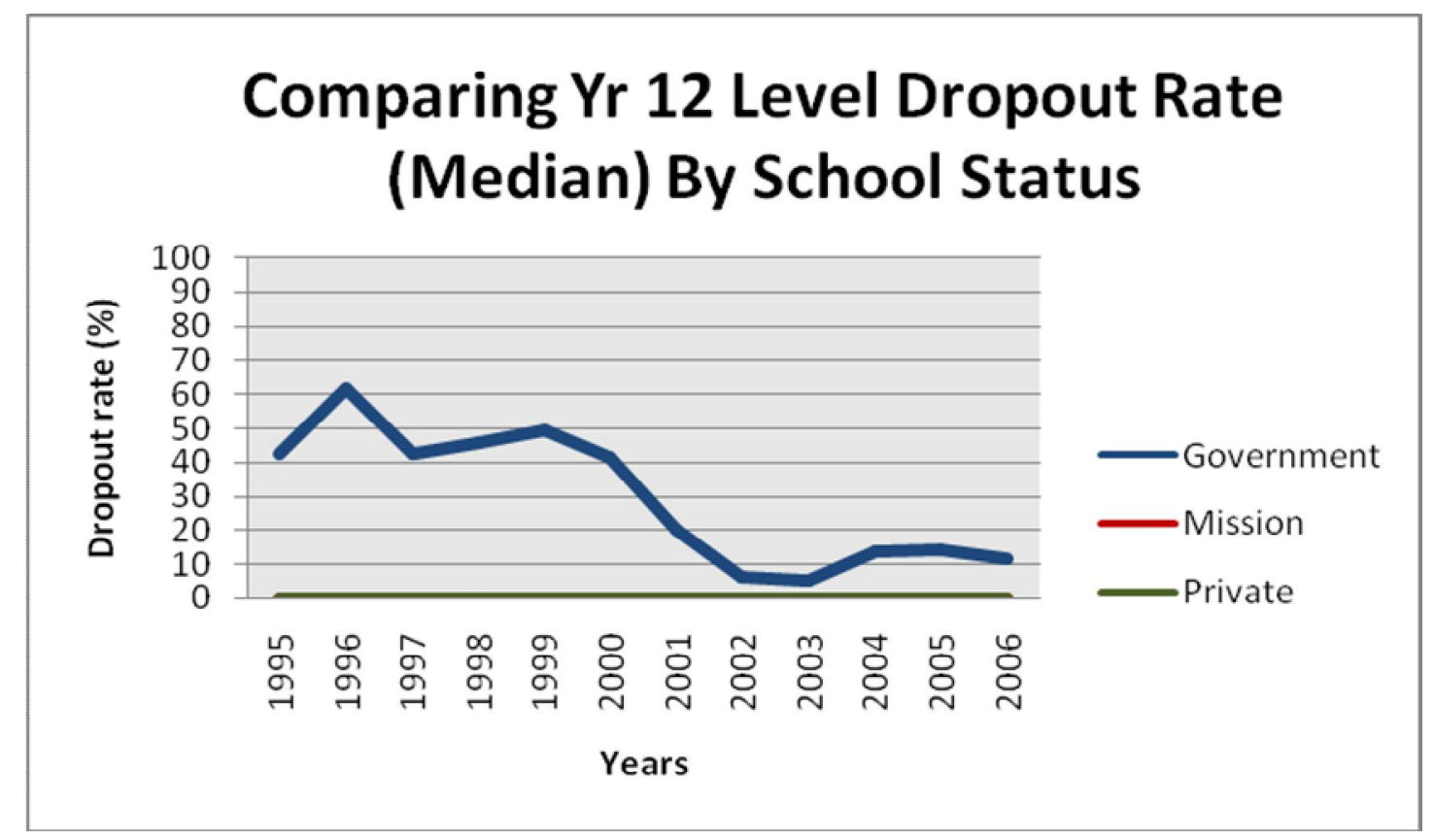

Figure 3.13: Comparing Year 12 Level Dropout Rates by School Status, 1995 2006

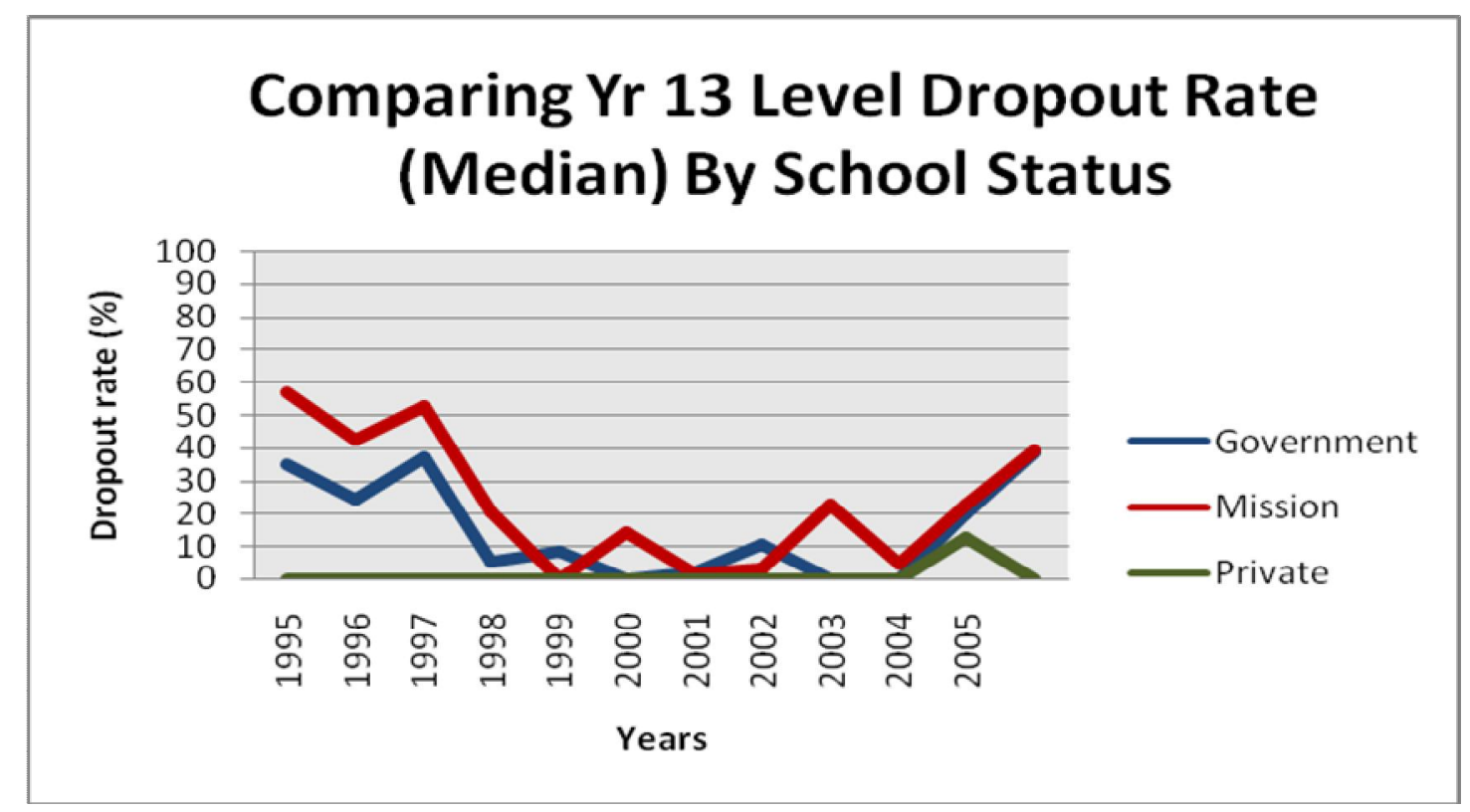

Figure 3.14: Comparing Year 13 Level Dropout Rates by School Status, 1995 2006

Much attention was given to the dropout rate in the Government schools as mentioned earlier in all the other school year levels, but at the Year 13 level the dropout crisis seems to be problematic for Mission schools as shown by Figure 3.14. Over the years the problem of students quiting schools has been improved across all the three school 
status, with the Mission schools are considered to have higher dropout rates than both the Government and Private schools. For Mission schools, some significant dropout rates were discovered in the year 2000, 2003, 2005 and 2006 but they are not as bad as in the late 90's. Similarly, the Government schools were not as bad either in the early $21^{\text {st }}$ century as it was in the late 90 's. One reason that could account for higher dropout rates in the late 90's is that there were only fewer secondary schools then than now. 


\section{Chapter 4}

\section{Data Analysis and Methodology}

This chapter is specifically discussing and talking about statistical theories, methodologies and approaches used to analyse the given datasets as described in chapter 3 for this study. This chapter was based on notes and informations from Agresti (2002), Hosmer \& Lemeshow (2000) and McCullagh \& Nelder (1989).

\subsection{Generalised Linear Models (GLMs)}

A GLM is a linear model that extends ordinary regression models to account for nonnormal response variables. The distributions of these response variables $\left\{Y_{i}, i=1, \ldots n\right\}$ must belong to the natural exponential family having the form

$$
\begin{aligned}
f\left(y_{i} ; \theta_{i}\right) & =s\left(y_{i}\right) t\left(\theta_{i}\right) \exp \left[a\left(y_{i}\right) b\left(\theta_{i}\right)\right] \\
& =\exp \left[a\left(y_{i}\right) b\left(\theta_{i}\right)+c\left(\theta_{i}\right)+d\left(y_{i}\right)\right] .
\end{aligned}
$$

where $b\left(\theta_{i}\right)$ is called the natural parameter. If $a\left(y_{i}\right)=y_{i}$ then (4.1) is in canonical form and hence becomes a natural member of the exponential family.

All generalised linear models have three components.

\section{Random Component}

The random component of a GLM identifies the response variable ' $Y_{i}$ ' with independent observations $\left(y_{1}, y_{2}, \ldots, y_{n}\right)$ and the probability distribution of ' $Y_{i}$ ' as in (4.1). For example, for the dropout dataset, the response variable is the number of dropouts, which follows a Binomial distribution. 


\section{Systematic Component}

The systematic component contains a set of explanatory variables $\left\{x_{i j}, j=1, \cdots p\right\}$ as follows:

$$
\eta_{i}=\sum_{j} \beta_{j} x_{i j}, \text { for } i=1, \ldots . . n \text { and } j=1, \ldots \ldots p
$$

Thus $\eta_{i}$ is referred to as the 'linear predictor' with the unknown set of parameters $\beta$. Usually, the coefficient of the intercept in the model is often denoted by ' $\alpha$ '.

In matrix form, (4.2) can be written as $\boldsymbol{\eta}=\boldsymbol{X} \boldsymbol{\beta}$, where $\boldsymbol{\eta}=\left(\eta_{1}, \ldots, \eta_{n}\right)^{T}, \boldsymbol{\beta}=\left(\beta_{1}, \ldots\right.$, $\left.\beta_{p}\right)^{T}$ are column vectors of model parameters. The model matrix $\mathbf{X}$ which is also called the design matrix is the $n \times p$ matrix of values of the explanatory variables for the $n$ observations or subjects.

\section{Link}

The third component of a GLM is the link function that relates the random and systematic components. It specifies how the expected value of the response variable from the natural exponential family $\mu_{i}=E\left(Y_{i}\right)$ connects to the linear predictor. In other words the model links $\mu_{i}$ to (4.2) by the link function $g\left(\mu_{i}\right)$, where $g(\cdot)$ is a monotonic, differentiable function.

The simplest link function is $g\left(\mu_{i}\right)=\mu_{i}$, called the identity link. This is the link function for ordinary regression models where the response variable $Y_{i}$ has a normal distribution. The link function that transforms the mean to the natural parameter is called the canonical link, that is $g\left(\mu_{i}\right)=b\left(\theta_{i}\right)$. Then (4.2) becomes

$$
b\left(\theta_{i}\right)=\sum_{j} \beta_{j} x_{i j}, \text { for } i=1, \ldots . . n \text { and } j=1, \ldots \ldots . p
$$

\subsubsection{Mean and Variance of a Random Variable}

Once a specified distribution of $Y_{i}$ is confirmed as a member of the natural exponential family, then we would be able to find its mean and variance as follows: 
Mean

The general form of the mean of a random variable is given by

$$
\mu_{i}=E\left[a\left(y_{i}\right)\right]=-\frac{c^{\prime}\left(\theta_{i}\right)}{b^{\prime}\left(\theta_{i}\right)}
$$

If the distribution of a random variable has canonical form then (4.4) becomes

$$
\mu_{i}=E\left[Y_{i}\right]=-\frac{c^{\prime}\left(\theta_{i}\right)}{b^{\prime}\left(\theta_{i}\right)}
$$

\section{Variance}

The general form of the variance of a random variable is given by

$$
\operatorname{Var}\left(a\left(y_{i}\right)\right)=\frac{c^{\prime}\left(\theta_{i}\right) b^{\prime \prime}\left(\theta_{i}\right)-c^{\prime \prime}\left(\theta_{i}\right) b^{\prime}\left(\theta_{i}\right)}{\left(b^{\prime}\left(\theta_{i}\right)\right)^{3}}
$$

If the distribution of a random variable has canonical form then (4.6) becomes

$$
\operatorname{Var}\left(Y_{i}\right)=\frac{c^{\prime}\left(\theta_{i}\right) b^{\prime \prime}\left(\theta_{i}\right)-c^{\prime \prime}\left(\theta_{i}\right) b^{\prime}\left(\theta_{i}\right)}{\left(b^{\prime}\left(\theta_{i}\right)\right)^{3}}
$$

where $c^{\prime}\left(\theta_{i}\right)$ is the first derivative of $c\left(\theta_{i}\right), b^{\prime}\left(\theta_{i}\right)$ is the first derivative of $b\left(\theta_{i}\right)$, $c^{\prime \prime}\left(\theta_{i}\right)$ is the second derivative of $c\left(\theta_{i}\right)$ and $b^{\prime \prime}\left(\theta_{i}\right)$ is the second derivative of $b\left(\theta_{i}\right)$.

\subsubsection{Maximum Likelihood Estimates (MLEs)}

The Maximum Likelihood method is often used by statisticians to estimate parameters for models. It maximises the likelihood of obtaining the observed set of data.

For a random variable with a pdf as in (4.1), the likelihood function $l(\beta)$ is the product of these pdfs: assuming independent observations. 


$$
\begin{aligned}
l(\beta) & =\prod_{i=1}^{n} f\left(y_{i} ; \theta_{i}\right) \\
& =\prod_{i=1}^{n} \exp \left[a\left(y_{i}\right) b\left(\theta_{i}\right)+c\left(\theta_{i}\right)+d\left(y_{i}\right)\right] \\
& =\exp \sum_{i=1}^{n}\left[a\left(y_{i}\right) b\left(\theta_{i}\right)+c\left(\theta_{i}\right)+d\left(y_{i}\right)\right]
\end{aligned}
$$

Taking the natural logarithm of the likelihood function (4.8), we obtain the log likelihood function:

$$
L(\beta)=\ln [l(\beta)]=\sum_{i=1}^{n}\left[a\left(y_{i}\right) b\left(\theta_{i}\right)+c\left(\theta_{i}\right)+d\left(y_{i}\right)\right]
$$

To find the value of $\boldsymbol{\beta}$ that maximises (4.9) we differentiate (4.9) with respect to $\boldsymbol{\beta}_{j}$ for $j=1, \cdots p$ and set it equal to zero.

$$
\frac{\partial L(\beta)}{\partial \beta_{j}}=\sum_{i} \frac{\partial L_{i}}{\partial \beta_{j}}=\sum_{i} \frac{\partial L_{i}}{\partial \theta_{i}} \frac{\partial \theta_{i}}{\partial \mu_{i}} \frac{\partial \mu_{i}}{\partial \eta_{i}} \frac{\partial \eta_{i}}{\partial \beta_{j}}=0
$$

Following the steps given by Agresti (2002, pages 135-136), the likelihood equations (4.10) can be reduced to

$$
S_{j}(\beta)=\sum_{i=1}^{n} \frac{\left(y_{i}-\mu_{i}\right)}{\operatorname{var}\left(Y_{i}\right)} x_{i j} \frac{\partial \mu_{i}}{\partial \eta_{i}}=0, \text { for } j=1, \ldots \ldots, p
$$

where $S_{j}(\beta)$ denotes the score function or the likelihood equations.

The MLEs of the unknown parameters $\boldsymbol{\beta}$ which are denoted by $\hat{\beta}$, are found by solving (4.11). 
For linear or ordinary regression, (4.11) is linear and hence it is easy to solve to get the estimators, $\hat{\beta}$. For a generalised linear model, (4.11) becomes non-linear and therefore requires numerical methods to solve it. Such methods include NewtonRaphson method and Fisher Scoring method (see Agresti, 2002 chapters 4 and 5 for more details on these methods).

\subsubsection{Quasi-Likelihood Estimates (QLEs)}

The Quasi-Likelihood estimates are found by solving the score functions (4.11) under the assumption of specifying the mean-variance relationship rather than the distribution of the response variable $Y_{i}$. This method is called the quasi-likelihood estimation. Under this approach, (4.11) is solved, but not based on the likelihood equations anymore, because the distribution of the response variable is not fully specified. We only need to specify the mean and variance of $Y_{i}$. The variance function $\operatorname{var}\left(Y_{i}\right)$ can be regarded as $v\left(\mu_{i}\right)$ which then changed (4.11) to

$$
S_{j}(\beta)=\sum_{i=1}^{n} \frac{\left(y_{i}-\mu_{i}\right)}{v\left(\mu_{i}\right)}\left(\frac{\partial \mu_{i}}{\partial \boldsymbol{\beta}}\right)^{T}=0, \text { for } j=1, \ldots \ldots, p
$$

where $\mu_{i}=E\left(Y_{i}\right)=g^{-1}\left(\sum_{j} \beta_{j} x_{i j}\right)=g^{-1}\left(\mathbf{X}_{i}^{T} \boldsymbol{\beta}\right), \quad\left(\frac{\partial \mu_{i}}{\partial \boldsymbol{\beta}}\right)^{T}=\left(\frac{\partial \mu_{i}}{\partial \beta_{1}}, \frac{\partial \mu_{i}}{\partial \beta_{2}}, \cdots \frac{\partial \mu_{i}}{\partial \beta_{p}}\right)$, and $\frac{\partial \mu_{i}}{\partial \beta_{j}}=\frac{\partial \mu_{i}}{\partial \eta_{i}} \frac{\partial \eta_{i}}{\partial \beta_{j}}=\frac{\partial \mu_{i}}{\partial \eta_{i}} x_{i j}$

The quasi-likelihood estimates $\hat{\boldsymbol{\beta}}$ are the solutions of the quasi-score equations (4.12).

\subsection{Logistic Regression Models}

For binary or dichotomous response variables in which only two possible outcomes, 'success' (taking value 1) and 'failure' (taking value 0), we assume the response variable follows a Binomial distribution. If the number of trials $\boldsymbol{n}=\boldsymbol{1}$ then the response variable has a Bernoulli distribution which is a special case of the binomial. 
For this study, the response variable $Y_{i}$ can be considered as the number of students who dropped out of school out of $\boldsymbol{n}$ students. We want to find out how the proportion of dropping out depends on a set of explanatory variables using logistic regression models. Since the random variable $Y_{i}$ has a Binomial distribution, then it has a probability function of the form:

$$
\begin{aligned}
f\left(y_{i}, \theta_{i}\right)= & \frac{n_{i} !}{y_{i} !\left(n_{i}-y_{i}\right) !} \theta_{i}^{y_{i}}\left(1-\theta_{i}\right)^{n_{i}-y_{i}} \\
& \propto\left(1-\theta_{i}\right)^{n_{i}} \exp \left[y_{i} \log \left(\frac{\theta_{i}}{1-\theta_{i}}\right)\right]
\end{aligned}
$$

where the natural parameter $b\left(\theta_{i}\right)=\log \left(\frac{\theta_{i}}{1-\theta_{i}}\right)$ is called the 'logit of $\theta_{i}$ ' or the log odds of ' dropping out'. Using the canonical link, the logistic regression model has the form

$$
\log \left(\frac{\theta_{i}}{1-\theta_{i}}\right)=\log i t\left(\theta_{i}\right)=\alpha+\beta_{1} x_{1}+\beta_{2} x_{2}+\ldots \ldots+\beta_{p} x_{p}
$$

\subsubsection{Parameters Interpretation}

In logistic regression models statisticians very often interpret the parameter $\boldsymbol{\beta}$ by means of odds ratio, probabilities and linear approximation. However, the latter is not being used in this study.

\section{Odds Ratio}

The odds ratio or the ratio of the odds is a measure of association used by statisticians to interpret the effect of a covariate or explanatory variable in a fitted logistic regression model. Suppose the model has only one explanatory variable $X$. The odds ratio is a comparison of the odds of success instead of failure for $x_{1}$ versus $x_{2}$, where $x_{1}$ and $x_{2}$ are two possible values of $x$. That is, the odds of success for $x_{1}$ is defined as $\frac{\theta_{i}\left(x_{1}\right)}{1-\theta_{i}\left(x_{1}\right)}$ and the odds of success for $x_{2}$ is defined as $\frac{\theta_{i}\left(x_{2}\right)}{1-\theta_{i}\left(x_{2}\right)}$, where $\theta_{i}\left(x_{1}\right)$ is the 
probability of success given $x_{1}$ and $\theta_{i}\left(x_{2}\right)$ is the probability of success given $x_{2}$. Therefore the odds ratio is defined as follows:

$$
\begin{aligned}
O R & =\frac{\operatorname{Odds}\left(x_{1}\right)}{\operatorname{Odds}\left(x_{2}\right)}=\frac{\frac{\theta_{i}\left(x_{1}\right)}{1-\theta_{i}\left(x_{1}\right)}}{\frac{\theta_{i}\left(x_{2}\right)}{1-\theta_{i}\left(x_{2}\right)}}=\exp \left(\left(\alpha+\beta \times x_{1}\right)-\left(\alpha+\beta \times x_{2}\right)\right) \\
& =\exp \left(\beta\left(x_{1}-x_{2}\right)\right)
\end{aligned}
$$

The value of the odds ratio must be greater than or equal to zero. If the value of the odds ratio becomes one (i.e $\mathrm{OR}=1$ ) then the covariate $X$ is independent of the response variable $Y$. However, if it is greater than one (i.e. $1<\mathrm{OR}<\infty$ ) then we say that the odds of success increases as $X$ increases. On the other hand, the odds of success decreases as $X$ increases if the odds ratio is less than one (i.e $0<\mathrm{OR}<1$ ).

\section{Probability}

The probability of success for certain levels of the covariate $X$ is calculated using

$\theta_{i}\left(\mathbf{X}_{i}\right)=\frac{\exp \left(\mathbf{X}_{i}{ }^{T} \beta\right)}{1+\exp \left(\mathbf{X}_{i}{ }^{T} \beta\right)}$

The calculation of the predicted probability of success is straight forward once we obtain the estimates of $\beta$.

\subsubsection{Inferences for Logistic Regression Models}

Very often, statisticians use three standard tests to test the significant of the null hypothesis $H_{0}: \beta=0$ for independence. These tests can be obtained by using most of statistical computer softwares, such as SAS. 


\section{Wald Test}

Under the null hypothesis with an asymptotic standard error (SE) of $\hat{\beta}$, $Z^{2}=\left(\frac{\hat{\beta}}{S E\left(\hat{\beta}_{j}\right)}\right)^{2}$ is asymptotically chi-squared $\left(\chi^{2}\right)$ distribution with 1 degree of freedom for a single parameter. For the multivariate case, it can be extended to $W=\left(\hat{\beta}-\beta_{0}\right)^{T}[\operatorname{Cov}(\hat{\beta})]^{-1}\left(\hat{\beta}-\beta_{0}\right)$.

The null hypothesis will be rejected if $W$ exceeds the critical value. In other words, the null hypothesis will be rejected if the p-value is less than the level of significance. The level of significance depends on the researcher; however the most common ones are $5 \%(0.05)$ and $1 \%(0.01)$. In most statistical packages a parameter with a p-value less than 0.001 is regarded as highly significant (which means that the corresponding explanatory variable is significantly associated with the response variable).

\section{Likelihood Ratio Test}

This test is based on the ratio of the two maximised log-likelihood functions $L_{0}$ and $L_{1}$. The function $L_{0}$ refers to the maximum value of the log-likelihood under $H_{0}$ while $L_{1}$ refers to maximum value of the log-likelihood for the full model $H_{0} \cup H_{1}$. The test statistic simplifies to $2\left(L_{1}-L_{0}\right)$.

The likelihood ratio test also has an asymptotic (when the sample size is large) chisquared distribution with $\operatorname{dim}\left(H_{0} \cup H_{1}\right)-\operatorname{dim}\left(H_{0}\right)$ degrees of freedom, under the null hypothesis.

\section{The Score Test}

The statistic of the score test for the univariate case is based on the partial derivatives of the $\log$-likelihood function $L(\beta)$ (as in (4.10) and (4.19)), which is $S(\beta)$ at $\beta_{0}$ under the null hypothesis, $H_{0}: \beta=\beta_{0}$, with an asymptotic chi-square distribution with degree of freedom, one ( $\left.\chi_{1}^{2}\right)$. That is the statistic is given by:

$$
\begin{aligned}
S(\beta) & =\frac{\left(U\left(\beta_{0}\right)\right)^{2}}{I\left(\beta_{0}\right)} \\
= & \frac{\left(\frac{\partial L\left(\beta_{0} \mid x\right)}{\partial \beta}\right)^{2}}{\frac{-\partial^{2} L\left(\beta_{0} \mid x\right)}{\partial \beta^{2}}}
\end{aligned}
$$


where $U(\beta)$ is the score function and $I(\beta)$ is the observed information or the variance of the score function (also known as Fisher information).

In the case of multivariate, the score test can be generalised into the form:

$$
U^{T}\left(\hat{\boldsymbol{\beta}}_{0}\right) I^{-1}\left(\hat{\boldsymbol{\beta}}_{0}\right) U\left(\hat{\boldsymbol{\beta}}_{0}\right) \quad \sim \chi_{p}^{2}
$$

that has an asymptotic chi-square distribution with $p$ degree of freedom under the null hypothesis, where $p$ is the number of constraints imposed on $\boldsymbol{\beta}$ by the null hypothesis. The $\hat{\boldsymbol{\beta}}_{0}$ is the maximum likelihood estimate of $\boldsymbol{\beta}$ under the null hypothesis and $U\left(\hat{\boldsymbol{\beta}}_{0}\right)=\frac{\partial L\left(\hat{\boldsymbol{\beta}}_{0} \mid x\right)}{\partial \boldsymbol{\beta}}$ and $I\left(\hat{\boldsymbol{\beta}}_{0}\right)=-E\left(\frac{\partial^{2} L\left(\hat{\boldsymbol{\beta}}_{0} \mid x\right)}{\partial \boldsymbol{\beta}^{2}}\right)$.

For this study we employ the score test based on the chi-square statistics.

\subsection{Model Selection}

Selecting the best statistical model from a set of potential ones to show and predict the effects of the predictors (explanatory or independent variables) on the response variable is not an easy task. There are various mathematical and statistical techniques that a researcher can employ in selecting the best model, however cautious must be taken. Very often the simple model is preferred over the full or complex one to avoid over parameterisation and over fitting the model. The full or complex model is the one with all the independent variables in it. The degree of complexity of the model is often done by counting the number of free parameters or variables in the model. Therefore, the best model selection technique will balance this degree of complexity and the goodness of fit of the model.

\subsubsection{Stepwise Regression}

This method is another variable selection procedure that some researchers or analysts often use to search for the best explanatory variables in any regression model. With this procedure, a variable may be added to or deleted from the model at each step. The procedure stops when no more predictors can be justifiably added or deleted based on the F-statistic, the t-statistic or the p-value criterion. The smaller the p-value (that is, less than the level of significance, say 0.05 or 0.01 as thresholds) the more significant an explanatory variable becomes and will be included in the model. 


\section{Forward Selection}

This procedure starts out by adding explanatory variables that are highly correlated with the response variable, one at a time. At each stage, the added variable will be tested and included in the model on the basis that it has a minimum p-value (less than the thresholds). The procedure stops when additional will not provide greatest improvement in fit significantly. In other words all the remaining variables in the model must have p-values less than the level of significance.

\section{Backward Elimination}

This procedure begins with the full or the complex model. At each stage it starts deleting or removing explanatory or independent variables that contribute the least to the model fitting based on the p-value. With this, the independent variable with the largest $\mathrm{p}$-value (greater than the thresholds or levels of significance) will be left out and can not re-enter at a later step. The procedure stops when remaining variables have $p$-values less than the cut off values or thresholds.

Many statisticians and researchers often use the backward elimination method for model selection because of its simplicity. This study will also use this tool for selecting the simple models as discussed in chapter 5 .

\subsubsection{Akaike Information Criterion (AIC)}

This is one of the model selection tools that many statisticians also use to select and compare competing models either nested or not. It selects the model that minimizes the expected Kullback-Leibler Information (also known as K-L distance) of the fitted model to the true model (Bozdogan, 2000). It also chooses the model with the lowest AIC.

$$
\begin{aligned}
A I C & =-2(\text { maximised } \log \text { Likelihood }- \text { number of parameters in the model }) \\
& =-2 L(\hat{\beta})+2 p
\end{aligned}
$$

where $p$ is the number parameters in the model and the term $2 p$ penalises for larger number of parameters. 
In previous sections, we focus on the situation where all observations are independent. However, many studies observe observations for each subject repeatedly. Sometimes, correlated observations can also occur when a set of observations is naturally formed as a cluster. In general, there are two approaches to deal with correlated observations. The marginal approach reflects the situation for the whole population, while the subject-specific approach is based on conditional models given each subject.

\subsubsection{Subject-Specific Approach}

The subject-specific approach gives a multilevel model. Sometimes, it is called Multilevel Analysis (Agresti, 2002; Goldstein, 1995), Hierarchical Analysis (Agresti, 2002; Antretter et al, 2005) or Random Coefficient Analysis (Twisk, Smidt \& de Ventre, 2005; Twisk, 2004; Mason, 2001). The main interest of the multilevel analysis is the inclusion of the random effects to account for subject-specific units correlations (Wang \& Louis, 2004) for repeated measurements of the outcome variable in the conditional model rather than just marginal (or population averaged).

A simple example to illustrate a subject-specific or conditional model (Masaoud \& Stryhn, 2010) would be considering the year level, the school, and the region as factors to measure student dropouts. With this we assume that student dropouts from the same year level, same school, and same region are more alike than student dropouts from different year level, different schools, and different region. This means that units within each cluster are correlated. Therefore the year level, school, and region are regarded as random effects. So basically the idea of using multilevel or random effects models is to see how the proability of student dropouts are related to various exaplanaory variables given a fixed year level, school, and region.

\subsubsection{Marginal Approach}

For the marginal approach or population average approach (Agresti, 2002; Masaoud \& Stryhn, 2010), the most popular method uses generalised estimating equations (GEE, Liang and Zeger, 1986). The GEE method is an extension of the quasilikelihood approach and it focuses on marginal average rather than subject levels (Agresti, 2002, \& Hanley et al, 2003). The estimates of $\boldsymbol{\beta}$ are found by extending (4.12) into multivariate cases as follows:

$$
S(\beta)=\sum_{i=1}^{n} \mathbf{D}_{i}^{T} \mathbf{V}_{i}^{-1} \mathbf{U}_{i}=0
$$


where $\mathbf{D}_{i}=\left(\frac{\partial \mu_{i}}{\partial \boldsymbol{\beta}}\right), \quad \mathbf{V}_{i}=\operatorname{Var}\left(\mathbf{Y}_{i}\right)$ the variance and covariance matrix of $\mathbf{Y}_{i}$ and $\mathbf{U}_{i}=\left(y_{i}-\mu_{i}\right)$.

The GEE method links each marginal mean to a linear predictor and provides a guess variance and covariance structure of $\mathbf{Y}_{i}$. For example, $\mathbf{Y}_{i}$ can be the numbers of dropouts for each of Year 2, .., Year 13, for school $i$. A possible working correlation has exchangeable structure that treats $\operatorname{Corr}\left(Y_{i s}, Y_{i t}\right)$ as identical for all possible $s$ and $t$. Then, the GEE method estimates the correlation using the assumed structure. The standard errors of model parameter estimates $\hat{\boldsymbol{\beta}}$ based on the assumed structure are updated using the information from the data. Therefore, even if the guess correlation structure is poor, the GEE method still provides robust standard errors for $\hat{\boldsymbol{\beta}}$. For more details about this method, see Agresti (2002) page 466-468 or Hosmer and Lemeshow (2000) page 313-314.

For the data, we do not have individual students' information. The dataset only has information on the dropout rate for each Year level within each school. It is not possible to find out whether the dropout is related to student's background (such as gender, social economic class, family structure, etc). Therefore the subject-specific approach is not appropriate. Instaed, the thesis uses the GEE approach to analyse the dropout rate in comparing the dropouts between Regions, School level and School status which is mainly focused on the marginal effects rather than the conditional effects.

Moreover, we choose the exchange correlation structure to analyse the data, based on the QIC (Quasilikelihood under the Independence model Criterion). The QIC criterion was first proposed by Pan (2001) as a model selection method and to select the best working correlation structure for GEE analyses. The model with the smallest QIC is preferred. The QIC formula was given by Cui (2007).

\subsection{Methodologies for the Dropout Survey Data}

This section talks about the statistical methods used to analyse the survey data which is the Samoan schools dropout rates for this research. We used the statistical computer software SAS Enteprise Guide 4.3 for all the statistical analysis. 


\section{Statistical Analysis}

This study used the Logistic Regression models to analyse the Samoan schools dropout rates. The given dropout rates were for year level only therefore estimates for the numbers of dropouts were calculated by using the formula:

$$
\text { Dropout Number }=\frac{\text { Percentage Dropout }}{100} \times \text { Year Level Size }
$$

The year level dropout rates are measured every year by the Ministry of Education, Sports and Culture (MESC) of Samoa through Census forms from the same schools. Notice that the year level refers to Year 1, Year 2,...,Year 13. For each calendar year (from 1995 to 2007), we use logistic regression models to find the relationship between the dropout rate and possible explanatory variables (such as region, year level, school level, and school status). Because the dropout rates are correlated within each school across different year levels, we use the GEE method to analyse such clustered data.

Among all the calendar years in this study (1995-2007), the interaction terms between region, year level, school level, school status, etc were not significant except for some few years. Therefore, we mostly focused on the main effects.

We used the backward elimination method to choose the best model by dropping insignificant explanatory variables based on the chi-square statistics. There is one thing we need to keep in mind. Some explanatory variables (predictors) were removed because of non-significance at the 5\% significance level. It does not mean that these variables were not related to the school dropout or dropout rate. It could be the result of multi-collinearity. This means that when these variables were highly correlated with other explanatory variables, it seemed that these variables were not important when all the others were in the model. This process was repeated until we found the simpler model whereby all the remaining variables were significantly associated with the response variable.

The effects of the explanatory variables on the response variable of selected models were explained or interpreted in terms of odds ratios. The effect of one variable was explained while keeping others fixed. The odds ratio was calculated using the formula (4.15) which was the exponential of the estimate of a particular variable. 


\section{Chapter 5}

\section{Results}

The chapter 3 shows the summarised dropout information across different explanatory variables. This chapter reports on findings about the association of the response variable (dropping out of school), and the selected explanatory variables based on models, where the models take the correlations into account and give the statistical inference on the effects of these explanatory variables. All models reported here were analysed separately by calendar year (1995 - 2006) for section 5.1 to 5.5 and 2007 for 5.6 to 5.8 due to reasons explained in chapters 1 and 3.

Section 5.1 will talk about the association of the response variable and the school year level and region. Again, the school year level is referring to Year 2 (denoted by Yr2) up to Year 13 (denoted by Yr13) and region is referring to Apia Urban, Rest of Upolu and Savaii. The Year 9 level was dropped from the analysis as we treated it being the first grade (year level) at Secondary school just like the Year 1 for Primary schools. There is no dropout information for the first year/grade in the school. This section will also report on which one of the three regions where students are more likely to give up schools.

In section 5.2, we will present the results of the association of dropping out of school and school year level and school level. The school level is comprised of Primary, Primary/Secondary and Secondary schools. There are 11 school year levels used in this study which started from Year 2 (Yr 2) up to Year 13 (Yr 13). The year levels Year 2 (Yr 2) to Year 8 (Yr 8) are for Primary Schools while Year 10 (Yr 10) to Year 13 (Yr 13) (an equivalent to Form 6) are for Secondary Schools as explained in chapter one.

Section 5.3 will display the association of dropping out of school and school year level and school status. Like the region and school level, the school status has three levels Government, Mission and Private. 
For each calendar year, we employed the GEE-approach to model both the main effects and interaction effects of the given explanatory variables. If the interaction effect is not significant then we drop it from the model.

The model selection was done using the backward elimination method, based on the type 3 Chi-Square statistics. An explanatory variable with a p-value of less than 0.05 (or 5\% significance level) will be kept otherwise it will be removed from the model.

In section 5.4, the study presents findings about the association of the response variable and the student-teacher ratio. The larger the school size in terms of student enrolments and fewer teachers, the higher this ratio is.

In section 5.5, the school size as in total school enrolment (TotEnrol) effects on the dropout will be covered here.

Section 5.6 reports on findings of the association of the secondary school teacher variables and the school dropouts for 2007 only. The secondary school teacher variables consisted of the proportion of female teachers; the proportion of Samoan teachers; the number of teachers with certificates and the number of teachers with degrees. The interpretation of the parameters is also done by odds ratio, which is the exponential of these parameter estimates.

The effect of school building variables on the dropout will be the focus of section 5.7. The findings in this section were based on 2007 secondary schools data only.

The last section (5.8) of this chapter reports on findings about the effects of the school facility variables on the school dropout or dropout rates for all the secondary schools in 2007.

\subsection{The Year Level and Region Effects}

The study found that the response variable (dropping out of school), was significantly associated with school year level (as in Yr 2 up to Yr 13) for all the calendar years $(1995,1996, \ldots \ldots, 2006)$ and region, for the following years 1995, 1996, 1997, 1998, 1999, 2001, 2003 and 2006. The test was based on the Chi-Squared statistics which tested the null hypothesis that there is no year level effect at 5\% significant level. We also tested the null hypothesis that there is no region effect, also based on the ChiSquared statistics at $5 \%$ level of significance. If an explanatory variable has a p-value less than this level of significance, $5 \%$ (or 0.05 ), then is believed to be siginificantly associated with the response variable. The variable region is referred to Apia urban, Rest of Upolu and Savaii (see chapter 1 and 3 for details).

For simplicity, the model written in words is:

$$
\text { Logit }(\text { dropout })=\text { year level }+ \text { region }
$$

The detailed model using the parameters notation is as follows: 
$\operatorname{Logit}\left(\pi_{i j}\right)=\alpha+\beta_{i}{ }^{X}+\beta_{j}{ }^{R}$, for $i=1,2, \ldots \ldots . ., 11$ and $j=1,2,3$.

where $\alpha=$ int ercept

$\beta_{i}^{X}=$ coefficient of yearlevel at the ith level

$\beta_{j}{ }^{R}=$ coefficient of region at the jth level

$\pi_{i j}=$ dropout rate for ith yearlevel and jth region

In SAS, the model set the Yr 8 year level and Savaii region as reference (or baseline) levels. The same goes for 2007, however there were only five year levels compare to twelve of the years mentioned above. This was due to the fact that only the secondary school enrolment figures were available at the time of data collection.

\section{Model Interpretation}

In 1995, dropping out of school was significantly (p-values < 0.05) associated with the school year level and region shown in Table 5.1. When the region effect is kept constant, the Yr2, Yr 10 and Yr 13 levels were significantly (p-values < 0.05) different from $\mathrm{Yr}$ 8. This means that students were more likely to drop out from these school year levels than those in $\mathrm{Yr}$ 8. The odds of dropping out in $\mathrm{Yr} 13$ was about 7.1 $(\mathrm{OR}=7.0958)$ times higher than that of Yr 8. For Yr 10 and Yr 2, the odds of dropping out were about $2.62(\mathrm{OR}=2.6172)$ and $2.38(\mathrm{OR}=2.3831)$ times higher than that of Yr 8 respectively. Other school year levels were not significantly different from $\operatorname{Yr} 8$. The odd ratio for $\operatorname{Yr} 8$ was set at 1 as a reference or baseline level.

When the school year level effect is held fixed, the Apia Urban region was significantly (p-value < 0.05) different from the Savaii region as shown in Table 5.1. That is, students were less likely to drop out of school in the Apia Urban than those in the Savaii region. In other words, Savaii students were more likely to drop out of school than those in the Apia Urban region. The odds of dropping out in the Savaii region was about $2.02(\mathrm{OR}=2.0178$, which is the inverse of 0.4956$)$ times higher than that of the Apia Urban region. The Rest of Upolu region was not significantly different from Savaii which means the odd of dropping out of school in the Rest of Upolu was also higher than that of the Apia Urban region.

The study also found significant associations between the response variable (dropout) and the school year level as well as the region for calendar years 1996, 1997, 1998, 1999 and 2001. These results are consistent with the preliminary results shown in chapter 3 and are found in Appendix A. 
Table 5.1: Estimates and Odds Ratio for Year Level and Region in 1995

\begin{tabular}{|c|c|c|c|c|c|}
\hline \multicolumn{5}{|c|}{ Analysis Of GEE Parameter Estimates } & \multirow[b]{3}{*}{$\begin{array}{l}\text { Odds } \\
\text { Ratio }\end{array}$} \\
\hline \multicolumn{5}{|c|}{ Empirical Standard Error Estimates } & \\
\hline Parameter & Level & Estimate & $\begin{array}{l}\text { Standard } \\
\text { Error }\end{array}$ & $\begin{array}{l}\text { P-Value } \\
(\operatorname{Pr}>|Z|)\end{array}$ & \\
\hline Intercept & & -3.214 & 0.1924 & $<.0001$ & \\
\hline YearLevel & Yr10 & 0.9621 & 0.2244 & $<.0001$ & 2.6172 \\
\hline YearLevel & Yr11 & 0.4453 & 0.471 & 0.3445 & 1.5610 \\
\hline YearLevel & Yr12 & -0.0147 & 1.052 & 0.9888 & 0.9854 \\
\hline YearLevel & Yr13 & 1.9595 & 0.7193 & 0.0064 & 7.0958 \\
\hline YearLevel & Yr2 & 0.8684 & 0.2223 & $<.0001$ & 2.3831 \\
\hline YearLevel & Yr3 & 0.0352 & 0.2276 & 0.8772 & 1.0358 \\
\hline YearLevel & Yr4 & -0.0221 & 0.2293 & 0.9231 & 0.9781 \\
\hline YearLevel & Yr5 & 0.2478 & 0.2149 & 0.2488 & 1.2812 \\
\hline YearLevel & Yr6 & 0.0561 & 0.2169 & 0.7958 & 1.0577 \\
\hline YearLevel & Yr7 & 0.232 & 0.2127 & 0.2755 & 1.2611 \\
\hline YearLevel & Yr8 & 0 & 0 & & 1.0000 \\
\hline Region & Apia Urban & -0.7019 & 0.2232 & 0.0017 & 0.4956 \\
\hline Region & Rest of Upolu & 0.0532 & 0.1599 & 0.7394 & 1.0546 \\
\hline Region & Savaii & 0 & 0 & & 1.0000 \\
\hline
\end{tabular}

\begin{tabular}{|l|r|r|r|}
\hline \multicolumn{4}{|c|}{ Score Statistics For Type 3 GEE Analysis } \\
\hline Source & DF & $\begin{array}{c}\text { Chi- } \\
\text { Square }\end{array}$ & $\begin{array}{c}\text { P-Value } \\
\text { (Pr > ChiSq) }\end{array}$ \\
\hline YearLevel & 10 & 36.97 & $<.0001$ \\
Region & 2 & 12.06 & 0.0024 \\
\hline
\end{tabular}

There was no association between dropping out of school and region (p-value $>0.05)$ in calendar years 2000, 2002, 2004 and 2005, but there was a significant association with the school year level ( $\mathrm{p}$-value $<0.0001$ ) for all these years based on the ChiSquare statistics. Nevertheless, for all these years students were more likely to dropout of school in rural areas, Savaii and Rest of Upolu regions. The full results and models are found in Appendix A.

Table 5.2: Chi-Square Statistics of the Model for Year Level and Region in 2000

\begin{tabular}{|l|r|rr|}
\hline \multicolumn{4}{|c|}{ Score Statistics For Type 3 GEE Analysis } \\
\hline Source & DF & $\begin{array}{c}\text { Chi- } \\
\text { Square }\end{array}$ & $\begin{array}{c}\text { P-Value } \\
\text { (Pr > ChiSq) }\end{array}$ \\
\hline YearLevel & \multicolumn{1}{|c}{4} & 43.37 & $<.0001$ \\
Region & 2 & 2.31 & 0.3156 \\
\hline
\end{tabular}


In 2003 dropping out of school was significantly (p-values < 0.05) associated with the school year level and region as shown by the Chi-Square statistics in Table 5.3. The only school year levels that were significantly (p-values < 0.05 ) different from Yr 8 were Yr 10, Yr 11, Yr 13, Yr 2 and Yr 7. The odds of dropping out of these school year levels were $1.8(\mathrm{OR}=1.8020), 2.83(\mathrm{OR}=2.8332), 3.14(\mathrm{OR}=3.1431), 1.75$ $(\mathrm{OR}=1.7528)$ and $1.56(\mathrm{OR}=1.5610)$ times higher than that of $\mathrm{Yr} 8$ respectively. With the significant school year levels, students were more likely to quit school as compared to those in Yr 8 and other insignificant school year levels when the region effect is being kept constant.

By keeping the school year level fixed, the Apia Urban was significantly (p-value < 0.05) different from the Savaii region. The Rest of Upolu was not significantly different from the Savaii region. Again, students were less likely to quit school in the Apia Urban region as compared to those of the Savaii and the Rest of Upolu regions. The odd of quitting school in the Savaii region was 1.47 (OR $=1.4717$, which is the inverse of 0.6795) times higher than that of the Apia Urban region.

Table 5.3: Estimates and Odds Ratio for Year Level and Region in 2003

\begin{tabular}{|l|l|r|r|r|r|}
\hline \multicolumn{5}{|c|}{ Analysis Of GEE Parameter Estimates } & \multirow{2}{*}{} \\
\cline { 1 - 4 } Parameter & Level & Estimate & $\begin{array}{c}\text { Standard } \\
\text { Error }\end{array}$ & $\begin{array}{c}\text { P-Value } \\
(\text { Pr }>|Z|)\end{array}$ & $\begin{array}{l}\text { Odds } \\
\text { Ratio }\end{array}$ \\
\hline Intercept & & -3.3708 & 0.2122 & $<.0001$ & \\
YearLevel & Yr10 & 0.5889 & 0.3003 & 0.0499 & 1.8020 \\
YearLevel & Yr11 & 1.0414 & 0.332 & 0.0017 & 2.8332 \\
YearLevel & Yr12 & 0.7503 & 0.4736 & 0.1131 & 2.1176 \\
YearLevel & Yr13 & 1.1452 & 0.5666 & 0.0433 & 3.1431 \\
YearLevel & Yr2 & 0.5612 & 0.228 & 0.0138 & 1.7528 \\
YearLevel & Yr3 & 0.2636 & 0.2201 & 0.2312 & 1.3016 \\
YearLevel & Yr4 & -0.2693 & 0.2384 & 0.2586 & 0.7639 \\
YearLevel & Yr5 & 0.2578 & 0.2299 & 0.2621 & 1.2941 \\
YearLevel & Yr6 & -0.0969 & 0.2881 & 0.7367 & 0.9076 \\
YearLevel & Yr7 & 0.4453 & 0.2099 & 0.0338 & 1.5610 \\
YearLevel & Yr8 & 0 & 0 &. & 1.0000 \\
Region & Apia Urban & -0.3864 & 0.1952 & 0.0477 & 0.6795 \\
Region & Rest of Upolu & 0.1463 & 0.1775 & 0.4097 & 1.1575 \\
Region & Savaii & 0 & 0 &. & 1.0000 \\
\hline
\end{tabular}

\begin{tabular}{|l|r|r|r|}
\hline \multicolumn{4}{|c|}{ Score Statistics For Type 3 GEE Analysis } \\
\hline Source & DF & $\begin{array}{c}\text { Chi- } \\
\text { Square }\end{array}$ & $\begin{array}{c}\text { P-Value } \\
\text { (Pr }>\text { ChiSq) }\end{array}$ \\
\hline YearLevel & 10 & 38.71 & $<.0001$ \\
Region & 2 & 7.46 & 0.024 \\
\hline
\end{tabular}


The Model for 2006 presented in Table 5.4 saw the interaction term, region $\times$ school year level, being significant ( $\mathrm{p}$-value $<0.05$ ), based on the Chi-Square statistics, so we kept it in the model. We also found that dropping out of school was significantly (p-values $<0.05)$ associated with the school year level and region.

For the main effects, we found that only Yr 13, Yr 2, Yr 4 and $\operatorname{Yr} 7$ were significantly (p-values < 0.05) different from $\operatorname{Yr} 8$. That is, more dropouts were likely to be found in Yr 13 and Yr 2 as compared to those in Yr 8 and other school year levels. On the other hand, even though students were less likely to quit school in Yr 8 as compared to Yr 13 and Yr 2, but it appears that students were more likely to quit school in Yr 8 than in Yr 4 and Yr 7. The Yr 8 was set as the reference level. By keeping the region and the interaction term fixed, we found that the odds of dropping out in $\mathrm{Yr} 13$ and $\mathrm{Yr}$ 2 were $5.48(\mathrm{OR}=5.4756)$ and $1.94(\mathrm{OR}=1.9420)$ times higher than that of $\mathrm{Yr} 8$ respectively. On the other hand, the odd of dropping out in Yr 8 was 2.29 (OR = 2.2878, which is the inverse of 0.4371) times higher than that of $\mathrm{Yr} 4$. Further, it was $2.1(\mathrm{OR}=2.094$, which is the inverse of 0.4952$)$ times higher than that of $\operatorname{Yr} 7$.

There was no significant difference found among all the three regions for the region main effects. However it became significant with the interaction term.

For the interaction effects, by keeping both the school year level and region effects constant, students were more likely to dropout of Yr 11 (p-value < 0.05) in the Apia Urban region compared to those of the same school year level in the Savaii and Rest of Upolu regions. The odd of quitting school was $7(\mathrm{OR}=7.0006)$ times higher. On the same note, students were more likely to dropout of Yr 3 (p-value $<0.05$ ) in the Apia Urban region compared to those of the same school year level in the Savaii and Rest of Upolu regions. The odd of this to happen was $2.7(\mathrm{OR}=2.6861)$ times higher.

Table 5.4: Estimates and Odds Ratio for Year Level and Region in 2006

\begin{tabular}{|c|c|c|c|c|c|c|}
\hline \multicolumn{6}{|c|}{ Analysis Of GEE Parameter Estimates } & \multirow[b]{3}{*}{$\begin{array}{l}\text { Odds } \\
\text { Ratio }\end{array}$} \\
\hline \multicolumn{6}{|c|}{ Empirical Standard Error Estimates } & \\
\hline Parameter & Level1 & Level2 & Estimate & $\begin{array}{l}\text { Standard } \\
\text { Error }\end{array}$ & $\begin{array}{l}\text { P-Value } \\
(\operatorname{Pr}>|Z|)\end{array}$ & \\
\hline Intercept & & & -2.7113 & 0.2535 & $<.0001$ & \\
\hline YearLevel & Yr10 & & 0.2464 & 0.3772 & 0.5136 & 1.2794 \\
\hline YearLevel & Yr11 & & -0.673 & 0.5612 & 0.2304 & 0.5102 \\
\hline YearLevel & Yr12 & & -0.4315 & 0.6478 & 0.5053 & 0.6495 \\
\hline YearLevel & Yr13 & & 1.7003 & 0.5126 & 0.0009 & 5.4756 \\
\hline YearLevel & Yr2 & & 0.6637 & 0.3378 & 0.0494 & 1.9420 \\
\hline YearLevel & Yr3 & & -0.1737 & 0.2923 & 0.5523 & 0.8405 \\
\hline YearLevel & Yr4 & & -0.8276 & 0.3444 & 0.0162 & 0.4371 \\
\hline YearLevel & Yr5 & & -0.5119 & 0.3367 & 0.1285 & 0.5994 \\
\hline YearLevel & Yr6 & & -0.1519 & 0.3855 & 0.6936 & 0.8591 \\
\hline
\end{tabular}




\begin{tabular}{|c|c|c|c|c|c|c|}
\hline YearLevel & Yr7 & & -0.7028 & 0.3286 & 0.0325 & 0.4952 \\
\hline YearLevel & Yr8 & & 0 & 0 & & 1.0000 \\
\hline Region & Apia Urban & & -0.7108 & 0.418 & 0.089 & 0.4913 \\
\hline Region & Rest of Upolu & & 0.1181 & 0.328 & 0.7189 & 1.1254 \\
\hline Region & Savaii & & 0 & 0 & & 1.0000 \\
\hline Region*YearLevel & Apia Urban & Yr10 & 0.3872 & 0.6642 & 0.56 & 1.4729 \\
\hline Region*YearLevel & Apia Urban & Yr11 & 1.946 & 0.8059 & 0.0157 & 7.0006 \\
\hline Region*YearLevel & Apia Urban & Yr12 & 0.9506 & 1.1451 & 0.4064 & 2.5873 \\
\hline Region*YearLevel & Apia Urban & Yr13 & 0.0339 & 0.7192 & 0.9624 & 1.0345 \\
\hline Region*YearLevel & Apia Urban & Yr2 & 0.3017 & 0.4972 & 0.544 & 1.3522 \\
\hline Region*YearLevel & Apia Urban & Yr3 & 0.9881 & 0.5026 & 0.0493 & 2.6861 \\
\hline Region*YearLevel & Apia Urban & Yr4 & 0.7634 & 0.5648 & 0.1764 & 2.1456 \\
\hline Region*YearLevel & Apia Urban & Yr5 & 0.6053 & 0.614 & 0.3242 & 1.8318 \\
\hline Region*YearLevel & Apia Urban & Yr6 & 0.5343 & 0.5793 & 0.3563 & 1.7063 \\
\hline Region*YearLevel & Apia Urban & Yr7 & 0.8445 & 0.5766 & 0.143 & 2.3268 \\
\hline Region*YearLevel & Apia Urban & Yr8 & 0 & 0 & & 1.0000 \\
\hline Region*YearLevel & Rest of Upolu & Yr10 & 0.177 & 0.5268 & 0.7368 & 1.1936 \\
\hline Region*YearLevel & Rest of Upolu & Yr11 & 1.0223 & 0.697 & 0.1424 & 2.7796 \\
\hline Region*YearLevel & Rest of Upolu & Yr12 & 0.8971 & 0.7852 & 0.2532 & 2.4525 \\
\hline Region*YearLevel & Rest of Upolu & Yr13 & 0.7923 & 0.6915 & 0.2519 & 2.2085 \\
\hline Region*YearLevel & Rest of Upolu & Yr2 & -0.6572 & 0.4416 & 0.1367 & 0.5183 \\
\hline Region*YearLevel & Rest of Upolu & Yr3 & -0.045 & 0.4121 & 0.913 & 0.9560 \\
\hline Region*YearLevel & Rest of Upolu & Yr4 & 0.5641 & 0.4479 & 0.2078 & 1.7579 \\
\hline Region*YearLevel & Rest of Upolu & Yr5 & 0.1469 & 0.4212 & 0.7273 & 1.1582 \\
\hline Region*YearLevel & Rest of Upolu & Yr6 & -0.0093 & 0.4652 & 0.984 & 0.9907 \\
\hline Region*YearLevel & Rest of Upolu & Yr7 & 0.6164 & 0.3831 & 0.1076 & 1.8522 \\
\hline Region*YearLevel & Rest of Upolu & Yr8 & 0 & 0 & & 1.0000 \\
\hline Region*YearLevel & Savaii & Yr10 & 0 & 0 & & 1.0000 \\
\hline Region*YearLevel & Savaii & Yr11 & 0 & 0 & & 1.0000 \\
\hline Region*YearLevel & Savaii & Yr12 & 0 & 0 & & 1.0000 \\
\hline Region*YearLevel & Savaii & Yr13 & 0 & 0 & & 1.0000 \\
\hline Region*YearLevel & Savaii & Yr2 & 0 & 0 & & 1.0000 \\
\hline Region*YearLevel & Savaii & Yr3 & 0 & 0 & & 1.0000 \\
\hline Region*YearLevel & Savaii & Yr4 & 0 & 0 & & 1.0000 \\
\hline Region*YearLevel & Savaii & Yr5 & 0 & 0 & & 1.0000 \\
\hline Region*YearLevel & Savaii & Yr6 & 0 & 0 & & 1.0000 \\
\hline Region*YearLevel & Savaii & Yr7 & 0 & 0 & & 1.0000 \\
\hline Region*YearLevel & Savaii & Yr8 & 0 & 0 & & 1.0000 \\
\hline
\end{tabular}




\begin{tabular}{|l|r|rr|}
\hline \multicolumn{4}{|c|}{ Score Statistics For Type 3 GEE Analysis } \\
\hline \multicolumn{1}{|c|}{ Source } & DF & \multicolumn{1}{c|}{$\begin{array}{c}\text { Chi- } \\
\text { Square }\end{array}$} & $\begin{array}{c}\text { P-Value } \\
\text { (Pr > ChiSq) }\end{array}$ \\
\hline YearLevel & 10 & 52.61 & $<.0001$ \\
Region & 2 & 9.85 & 0.0073 \\
Region*YearLevel & 20 & 36.14 & 0.0148 \\
\hline
\end{tabular}

\subsection{The School Level Effect}

The study found no significant association between dropout and School Level (Primary, Primary/Secondary and Secondary) across all the years, given the year level (Yr 2, Yr 3 up to Yr 13). The test was based on the null hypothesis that there is no School Level effect using Chi-Squared statistics for the GEE method. It is not surprised that we do not find significant effects for School Level, given the year level, because the School Level is highly correlated with the year level. However, it is still interesting to see the comparison between the three School Levels based on the odds ratio. Given the year levels students were more likely to drop out of Secondary schools than the Primary schools. As an example, Table 5.5 clearly shows that the odd of dropping out in the Secondary was 20.88 ( $O R=20.8768$, which is the inverse of 0.0479) times higher than that of Primary. For all the models, the Secondary school was set as a reference or baseline level. For simplicity, the model being used in words is as follows:

$$
\text { Logit }(\text { dropout rate })=\text { year level }+ \text { school level }
$$

This model could be transformed into parameters notation which is:

$$
\begin{aligned}
& \text { Logit }\left(\pi_{i j}\right)=\alpha+\beta_{i}{ }^{X}+\beta_{j}{ }^{L} \text {, for } i=1,2, \ldots \ldots . ., 11 \text { and } j=1,2,3 . \\
& \text { where } \alpha=\text { intercept } \\
& \qquad \begin{array}{l}
\beta_{i}{ }^{X}=\text { coefficient of year level at the } i \text { th level } \\
\beta_{j}{ }^{L}=\text { coefficient of school level at the } j \text { th level } \\
\pi_{i j}=\text { dropout rate for ith yearlevel and jth school level }
\end{array}
\end{aligned}
$$

The full results and models for school level effect are appeared in the Appendix A. 
Table 5.5: Estimates and Odds Ratio for Year Level and School Level in 1995

\begin{tabular}{|c|c|c|c|c|c|}
\hline \multicolumn{5}{|c|}{ Analysis Of GEE Parameter Estimates } & \multirow[b]{3}{*}{$\begin{array}{l}\text { Odds } \\
\text { Ratio }\end{array}$} \\
\hline \multicolumn{5}{|c|}{ Empirical Standard Error Estimates } & \\
\hline Parameter & Level & Estimate & $\begin{array}{l}\text { Standard } \\
\text { Error }\end{array}$ & $\begin{array}{c}\text { P-Value } \\
(\operatorname{Pr}>|Z|)\end{array}$ & \\
\hline Intercept & & -0.4012 & 0.6705 & 0.5495 & \\
\hline YearLevel & Yr10 & -2.0928 & 0.6449 & 0.0012 & 0.1233 \\
\hline YearLevel & Yr11 & -2.5825 & 0.8985 & 0.004 & 0.0756 \\
\hline YearLevel & Yr12 & -3.0366 & 1.2451 & 0.0147 & 0.0480 \\
\hline YearLevel & Yr13 & -1.1577 & 0.8033 & 0.1495 & 0.3142 \\
\hline YearLevel & Yr2 & 0.8921 & 0.2564 & 0.0005 & 2.4402 \\
\hline YearLevel & Yr3 & 0.0502 & 0.2598 & 0.8468 & 1.0515 \\
\hline YearLevel & Yr4 & 0.0202 & 0.256 & 0.937 & 1.0204 \\
\hline YearLevel & Yr5 & 0.2679 & 0.2403 & 0.265 & 1.3072 \\
\hline YearLevel & Yr6 & 0.0609 & 0.2565 & 0.8122 & 1.0628 \\
\hline YearLevel & Yr7 & 0.2755 & 0.2393 & 0.2497 & 1.3172 \\
\hline YearLevel & Yr8 & 0 & 0 & & 1.0000 \\
\hline SchoolLevel & Primary & -3.0385 & 0.6555 & $<.0001$ & 0.0479 \\
\hline SchoolLevel & Primary/Secondary & -1.1045 & 0.6463 & 0.0875 & 0.3314 \\
\hline SchoolLevel & Secondary & 0 & 0 & & 1.0000 \\
\hline
\end{tabular}

\begin{tabular}{|l|rrrr|}
\hline \multicolumn{5}{|c|}{ Score Statistics For Type 3 GEE Analysis } \\
\hline \multicolumn{1}{|c|}{ Source } & DF & $\begin{array}{c}\text { Chi- } \\
\text { Square }\end{array}$ & $\begin{array}{c}\text { P-Value } \\
\text { (Pr > ChiSq) }\end{array}$ \\
\hline YearLevel & & 10 & 26.42 & 0.0032 \\
SchoolLevel & & 2 & 2.2 & 0.333 \\
\hline
\end{tabular}

\subsection{The School Status Effect}

The study found significant ( $\mathrm{p}$-value $<0.05$ ) associations between dropping out and School Status for calendar years 1999, 2000 and 2003. The School Status refers to Government school, Mission school and Private school. The results are shown in Table 5.6 to Table 5.8. In each of these Tables the school year level Yr 8 and the School Status Private were set as reference or baseline levels. The results show that students were more likely to drop out of Government schools than those of both the Mission and Private schools. Full results are found in Appendix A. For simplicity, the model being used in words is as follows:

$$
\text { Logit }(\text { dropout rate })=\text { year level }+ \text { school status }
$$


This model could be transformed into parameters notation which is:

$$
\begin{aligned}
& \operatorname{Logit}\left(\pi_{i j}\right)=\alpha+\beta_{i}{ }^{X}+\beta_{j}{ }^{S}, \text { for } i=1,2, \ldots \ldots . ., 11 \text { and } j=1,2,3 . \\
& \text { where } \alpha=\text { int ercept } \\
& \qquad \begin{array}{l}
\beta_{i}{ }^{X}=\text { coefficient of yearlevel at the ith level } \\
\beta_{j}{ }^{S}=\text { coefficient of school status at the jth level } \\
\pi_{i j}=\text { dropout rate for ith yearlevel and jth school status }
\end{array}
\end{aligned}
$$

\section{Model Interpretation}

In 1999, the study found that dropping out of school was significantly (p-values < 0.05 ) associated with the School Status which is based on the Chi-Square statistics as shown in Table 5.6. For the School Status effect, when keeping the school year level

\begin{tabular}{|c|c|c|c|c|c|}
\hline \multicolumn{5}{|c|}{ Analysis Of GEE Parameter Estimates } & \multirow[b]{3}{*}{$\begin{array}{l}\text { Odds } \\
\text { Ratio }\end{array}$} \\
\hline \multicolumn{5}{|c|}{ Empirical Standard Error Estimates } & \\
\hline Parameter & Level & Estimate & $\begin{array}{l}\text { Standard } \\
\text { Error }\end{array}$ & $\begin{array}{c}\text { P-Value } \\
(\mathrm{Pr}>|\mathrm{Z}|)\end{array}$ & \\
\hline Intercept & & -4.5836 & 0.8515 & $<.0001$ & \\
\hline YearLevel & Yr10 & 1.6626 & 0.3777 & $<.0001$ & 5.2730 \\
\hline YearLevel & Yr11 & 1.2061 & 0.4611 & 0.0089 & 3.3404 \\
\hline YearLevel & Yr12 & 2.5367 & 0.4685 & $<.0001$ & 12.6379 \\
\hline YearLevel & Yr13 & 1.8062 & 0.6282 & 0.004 & 6.0873 \\
\hline YearLevel & Yr2 & 1.3842 & 0.2658 & $<.0001$ & 3.9916 \\
\hline YearLevel & Yr3 & 0.7118 & 0.2793 & 0.0108 & 2.0377 \\
\hline YearLevel & Yr4 & 0.8033 & 0.2494 & 0.0013 & 2.2329 \\
\hline YearLevel & Yr5 & 0.7993 & 0.2772 & 0.0039 & 2.2240 \\
\hline YearLevel & Yr6 & 0.6744 & 0.2607 & 0.0097 & 1.9629 \\
\hline YearLevel & Yr7 & 1.0738 & 0.2562 & $<.0001$ & 2.9265 \\
\hline YearLevel & Yr8 & 0 & 0 & & 1.0000 \\
\hline SchoolStatus & Government & 0.7368 & 0.8124 & 0.3644 & 2.0892 \\
\hline SchoolStatus & Mission & -0.8232 & 0.8867 & 0.3532 & 0.4390 \\
\hline SchoolStatus & Private & 0 & 0 & & 1.0000 \\
\hline
\end{tabular}
fixed and despite there was no significant difference between the three levels of School Status the odds ratio of the Government school $(\mathrm{OR}=2.0892)$ was higher than that of the baseline, Private school as shown in Table 5.6.

Table 5.6: Estimates and Odds Ratio for Year Level and School Status in 1999 


\begin{tabular}{|l|r|rr|}
\hline \multicolumn{4}{|c|}{ Score Statistics For Type 3 GEE Analysis } \\
\hline Source & DF & $\begin{array}{c}\text { Chi- } \\
\text { Square }\end{array}$ & $\begin{array}{c}\text { P-Value } \\
\text { (Pr }>\text { ChiSq) }\end{array}$ \\
\hline YearLevel & 10 & 41.39 & $<.0001$ \\
SchoolStatus & 2 & 11.18 & 0.0037 \\
\hline
\end{tabular}

In 2000, the study also found that dropping out of school was significantly (p-values < 0.05) associated with the School Status based on the Chi-Square Statistics in Table 5.7. Despite no significant difference was found between the three levels of School Status, but the odds ratio shows that more students were deemed to quit Government schools $(\mathrm{OR}=2.1611)$ than those of the Private schools.

Table 5.7: Estimates and Odds Ratio for Year Level and School Status in 2000

\begin{tabular}{|c|c|c|c|c|c|}
\hline \multicolumn{5}{|c|}{ Analysis Of GEE Parameter Estimates } & \multirow[b]{3}{*}{$\begin{array}{l}\text { Odds } \\
\text { Ratio }\end{array}$} \\
\hline \multicolumn{5}{|c|}{ Empirical Standard Error Estimates } & \\
\hline Parameter & Level & Estimate & $\begin{array}{l}\text { Standard } \\
\text { Error }\end{array}$ & $\begin{array}{l}\text { P-Value } \\
(\operatorname{Pr}>|\mathrm{Z}|)\end{array}$ & \\
\hline Intercept & & -4.1348 & 0.4476 & $<.0001$ & \\
\hline YearLevel & Yr10 & 1.3737 & 0.3131 & $<.0001$ & 3.9499 \\
\hline YearLevel & Yr11 & 0.8138 & 0.3851 & 0.0346 & 2.2565 \\
\hline YearLevel & Yr12 & 1.8086 & 0.4178 & $<.0001$ & 6.1019 \\
\hline YearLevel & Yr13 & 0.798 & 0.6521 & 0.2211 & 2.2211 \\
\hline YearLevel & Yr2 & 1.213 & 0.2291 & $<.0001$ & 3.3636 \\
\hline YearLevel & Yr3 & 0.3954 & 0.2309 & 0.0868 & 1.4850 \\
\hline YearLevel & Yr4 & 0.4904 & 0.2183 & 0.0247 & 1.6330 \\
\hline YearLevel & Yr5 & 0.674 & 0.2635 & 0.0105 & 1.9621 \\
\hline YearLevel & Yr6 & 0.6596 & 0.2205 & 0.0028 & 1.9340 \\
\hline YearLevel & Yr7 & 0.6783 & 0.2303 & 0.0032 & 1.9705 \\
\hline YearLevel & Yr8 & 0 & 0 & & 1.0000 \\
\hline SchoolStatus & Government & 0.7706 & 0.4195 & 0.0662 & 2.1611 \\
\hline SchoolStatus & Mission & 0.0277 & 0.4764 & 0.9536 & 1.0281 \\
\hline SchoolStatus & Private & 0 & 0 & & 1.0000 \\
\hline
\end{tabular}

\begin{tabular}{|l|r|r|r|}
\hline \multicolumn{4}{|c|}{ Score Statistics For Type 3 GEE Analysis } \\
\hline Source & DF & $\begin{array}{c}\text { Chi- } \\
\text { Square }\end{array}$ & $\begin{array}{c}\text { P-Value } \\
\text { (Pr > ChiSq) }\end{array}$ \\
\hline YearLevel & \multicolumn{4}{c}{42.82} & $<.0001$ \\
SchoolStatus & 2 & 7.27 & 0.0264 \\
\hline
\end{tabular}


The study found that dropping out of school was again significantly (p-values $<0.05$ ) associated with the School Status in 2003. This is based on the Chi-Square statistics shown in Table 5.8. Despite no significant difference was found between the three levels of School Status, but the odds ratio shows that students were more likely to leave Government schools $(\mathrm{OR}=2.1453)$ prematurely than those of the Private schools.

Table 5.8: Estimates and Odds Ratio for Year Level and School Status in 2003

\begin{tabular}{|l|l|r|r|r|r|}
\hline \multicolumn{5}{|c|}{ Analysis Of GEE Parameter Estimates } \\
\cline { 1 - 4 } Parameter & \multicolumn{1}{|c|}{ Level } & Estimate & $\begin{array}{c}\text { Standard } \\
\text { Error }\end{array}$ & $\begin{array}{c}\text { P-Value } \\
\text { (Pr }>|Z|)\end{array}$ & $\begin{array}{l}\text { Odds } \\
\text { Ratio }\end{array}$ \\
\hline Intercept & & -4.1202 & 0.7284 & $<.0001$ & \\
YearLevel & Yr10 & 0.6831 & 0.2719 & 0.012 & 1.9800 \\
YearLevel & Yr11 & 1.1407 & 0.3221 & 0.0004 & 3.1290 \\
YearLevel & Yr12 & 0.8773 & 0.4401 & 0.0462 & 2.4044 \\
YearLevel & Yr13 & 1.1952 & 0.5743 & 0.0374 & 3.3042 \\
YearLevel & Yr2 & 0.5896 & 0.2171 & 0.0066 & 1.8033 \\
YearLevel & Yr3 & 0.284 & 0.2085 & 0.1731 & 1.3284 \\
YearLevel & Yr4 & -0.2489 & 0.2286 & 0.2763 & 0.7797 \\
YearLevel & Yr5 & 0.2784 & 0.2191 & 0.2039 & 1.3210 \\
YearLevel & Yr6 & -0.0814 & 0.2805 & 0.7716 & 0.9218 \\
YearLevel & Yr7 & 0.4705 & 0.1988 & 0.018 & 1.6008 \\
YearLevel & Yr8 & 0 & 0 &. & 1.0000 \\
SchoolStatus & Government & 0.7633 & 0.699 & 0.2748 & 2.1453 \\
SchoolStatus & Mission & 0.0695 & 0.7258 & 0.9238 & 1.0720 \\
SchoolStatus & Private & 0 & 0 & & 1.0000 \\
\hline
\end{tabular}

\begin{tabular}{|l|r|r|r|}
\hline \multicolumn{4}{|c|}{ Score Statistics For Type 3 GEE Analysis } \\
\hline \multicolumn{1}{|c|}{ Source } & DF & $\begin{array}{c}\text { Chi- } \\
\text { Square }\end{array}$ & $\begin{array}{r}\text { P-Value } \\
\text { (Pr > ChiSq) }\end{array}$ \\
\hline YearLevel & 10 & 46.52 & $<.0001$ \\
SchoolStatus & 2 & 10.93 & 0.0042 \\
\hline
\end{tabular}

There was no significant relationship found between dropout and school status in other calendar years (1995, 1996, 1997, 1998, 2001, 2002, 2004, 2005 and 2006). The full results and models for these years are appeared in Appendix A. 
The Student-Teacher Ratio (STR) is one of the key indicators in education that helps measure the efficiency and quality (Ministry of Education Ethiopia, 2010) of education. According to UNESCO (2006), it is a common indicator in educational planning which is used to measure the level and estimate the required number of teachers. It is believed that higher student-teacher ratio is a result of large number of enrolments which leads to students receiving less attention from the teacher; thereby affect the teaching and learnig quality. On the other hand, lower student-teacher ratio is a consequence of smaller classes which in turn students get more attention and help and thereby improve the quality of education (Ministry of Education of Ethiopia, 2010). However, lower student-teacher ratio sometimes indicates low efficientcy of the education system as a result of under utilisation of teachers according to the Ministry of Education of Ethiopia (2010). As the literature says that dropping out of school is associated with the student-teacher ratio and the higher this ratio is the higher the dropout rates as well.

For this study, we created this new variable, the student-teacher ratio, from the existing explanatory variables, TotEnrol (Total Enrolment) and NumofTeachers (Total Number of Teachers in a school), by dividing the school total enrolment of each year by the total number of teachers of the same school of the same year.

We modelled the association of the dropout and student-teacher ratio by fitting in all the other explanatory variables to see if the student-teacher ratio has a relationship with. These explanatory variables include the region, school status and school level. Initially, the total enrolment was included in the model but because of its significant correlation with the student-teacher ratio, hence being dropped and excluded from the model. With regards to this exclusion we later modeled the effect of the Total enrolment on the dropouts separately.

The model selection was based on the Chi-Square statistics at 5\% (or 0.05) level of significance, using the Stepwise backward elimination. An explanatory variable with a higher $\mathrm{p}$-value ( $\mathrm{p}$-value $>0.05$ ) was then removed and re-ran the model again. There was no interaction term involved due to convergence difficulty.

Across all the calendar years, 1995 to 2006, we have found that dropping out of school was significantly associated with the student-teacher ratio. In all these years drop out was inversely related to student-teacher ratio when controlling the effect of other co-variates. In other words, students were less likely to leave school prematurely in schools with higher student-teacher ratio. Conversely, the lower the student-teacher ratio implies the higher the dropout rate. But higher student-teacher ratio also implies larger school enrolments. As a result of this negatively relationship, an in-depth analysis or detailed explanation is warranted to support why fewer dropouts were found in larger schools. As shown in section 5.1, the Rergion effects, fewer dropouts 
were found in the Apia Urban region as compared to those in the rural areas, Rest of Upolu and Savaii regions. The Apia Urban region has the highest number of enrolments and larger schools as compare to Rest of Upolu and Savaii regions. Having the highest number of enrolments, Apia Urban region also has the higher student-teacher ratio as shown by Figures 5.1 to 5.2. Furthermore, Apia Urban schools have more activities in which students are engaged in than schools in the Rest of Upolu and Savaii regions. Thus students were less likely to drop out of schools with higher student-teacher ratios as well as larger number of enrolments.

COMPARING THE STUDENT-TEACHER RATIO

BY REGION (1995)

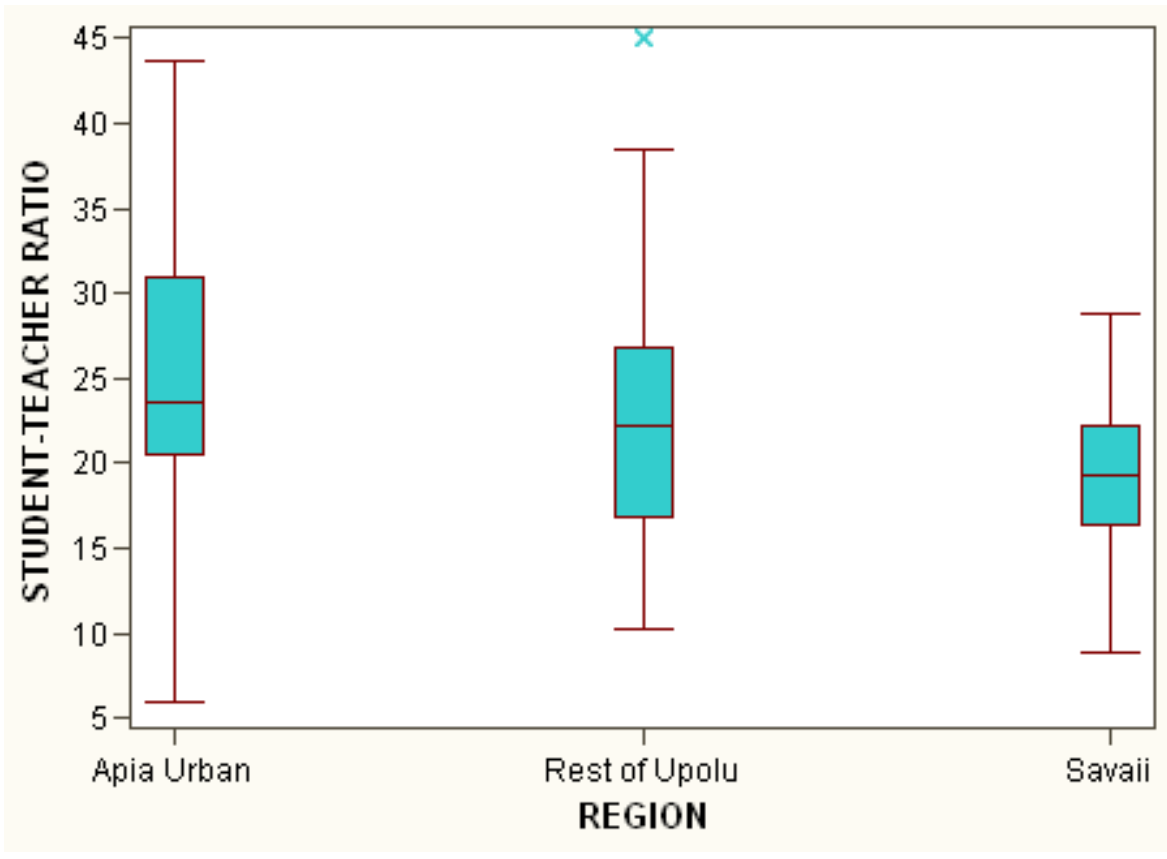

Figure 5.1: Distribution of the Student-Teacher Ratio by Region (1995) 


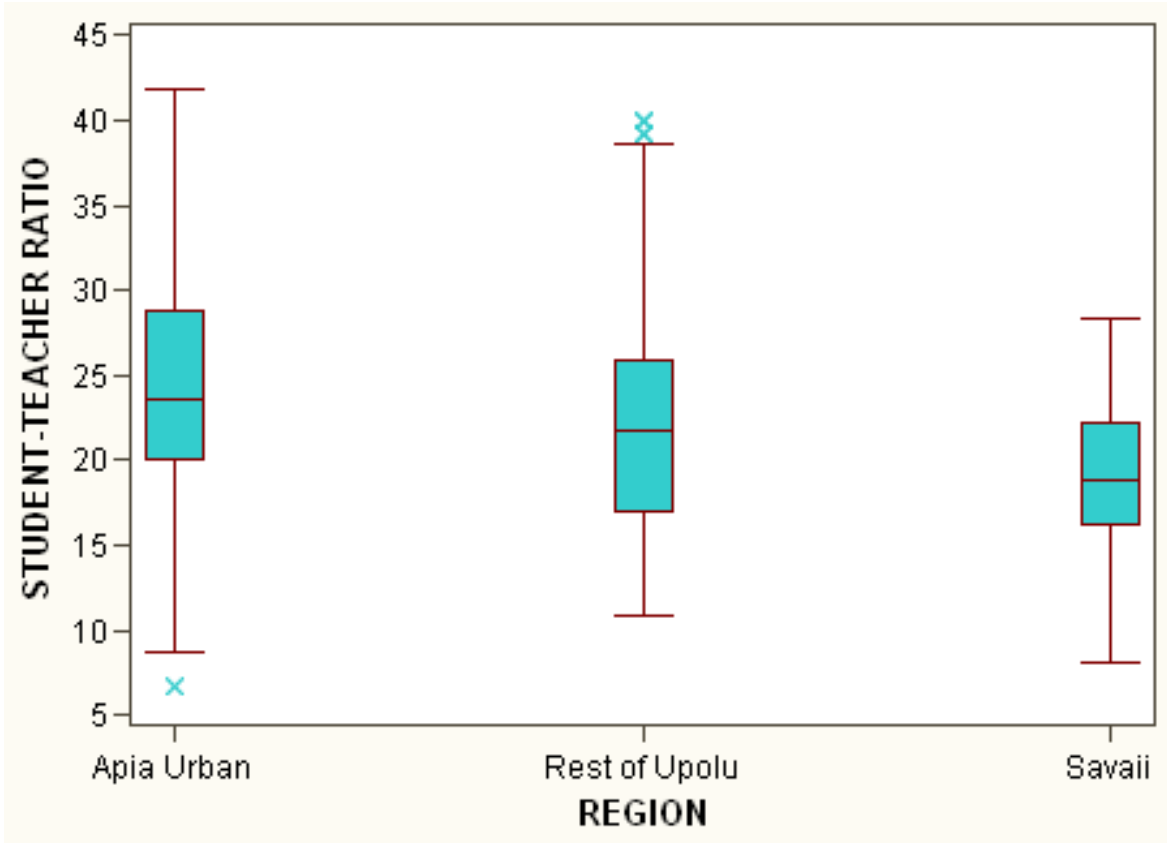

Figure 5.2: Distribution of the Student-Teacher Ratio by Region (1996)

\section{Model Interpretation}

As mentioned earlier that dropping out was significantly associated with the studentteacher ratio across all the calendar years. The covariate Total enrolment was excluded from the model due to its relationship with the student-teacher relationship. Other explanatory variables that were involved in selecting the more parsimonious model together with the student-teacher ratio were the region, school status and school level.

In 1995, we fitted the model for student-teacher ratio with the other three co-variates and only the student-teacher ratio was significantly associated with the dropout based on the Chi-Square statistics as shown in Table 5.9. Each of the other three covariates was removed from the model everytime we ran it until we had the final model presented in Table 5.10. The model shows that students were less likely to quit schools with higher student-teacher ratios. It was the same story for 1996 but this time only the school status was not significant as shown in Table 5.11. The final model for 1996 is presented in Table 5.12 when the school status was dropped and all the three covariates were all significant. 
Table 5.9: Chi-Square Statistics for the Model with four Co-variates (1995)

\begin{tabular}{|l|r|r|r|}
\hline \multicolumn{4}{|c|}{ Score Statistics For Type 3 GEE Analysis } \\
\hline \multicolumn{1}{|c|}{ Source } & DF & $\begin{array}{c}\text { Chi- } \\
\text { Square }\end{array}$ & $\begin{array}{c}\text { P-Value } \\
\text { (Pr > ChiSq) }\end{array}$ \\
\hline StudentTeachRatio & 1 & 8.45 & 0.0036 \\
Region & 2 & 3.66 & 0.1604 \\
SchoolStatus & 2 & 0.34 & 0.8451 \\
SchoolLevel & 2 & 2.57 & 0.2762 \\
\hline
\end{tabular}

Table 5.10: Final Model for the Student-Teacher ratio effect (1995)

\begin{tabular}{|c|c|c|c|c|}
\hline \multicolumn{4}{|c|}{ Analysis Of GEE Parameter Estimates } & \\
\hline \multicolumn{4}{|c|}{ Empirical Standard Error Estimates } & \\
\hline Parameter & Estimate & $\begin{array}{l}\text { Standard } \\
\text { Error }\end{array}$ & $\begin{array}{c}\text { P-Value } \\
(\operatorname{Pr}>|Z|)\end{array}$ & $\begin{array}{l}\text { Odds } \\
\text { Ratio }\end{array}$ \\
\hline Intercept & -1.9183 & 0.288 & $<.0001$ & \\
\hline StudentTeachRatio & -0.0454 & 0.0132 & 0.0006 & 0.9556 \\
\hline
\end{tabular}

\begin{tabular}{|c|c|c|r|}
\hline \multicolumn{4}{|c|}{ Score Statistics For Type 3 GEE Analysis } \\
\hline Source & DF & $\begin{array}{c}\text { Chi- } \\
\text { Square }\end{array}$ & $\begin{array}{c}\text { P-Value } \\
\text { (Pr > ChiSq) }\end{array}$ \\
\hline StudentTeachRatio & 1 & 9.27 & 0.0023 \\
\hline
\end{tabular}

When holding the student-teacher ratio and school level effects fixed, students were more likely to drop out of school in the Rest of Upolu than those in the Apia Urban and Savaii regions. The odd of dropping out in the Rest of Upolu region was 1.78 (OR $=1.7819$ ) times higher than that of the baseline, Savaii region. But when keeping the student-teacher ratio and region effects constant, students were less likely to drop out of Primary than the baseline, Secondary schools. The odd of quitting Secondary schools was $1.67(\mathrm{OR}=1.6675$, which is the inverse of 0.5997$)$ times higher than that of Primary schools.

Table 5.11: Chi-Square Statistics for the Model with four Co-variates (1996)

\begin{tabular}{|l|r|rr|}
\hline \multicolumn{4}{|c|}{ Score Statistics For Type 3 GEE Analysis } \\
\hline \multicolumn{1}{|c|}{ Source } & DF & $\begin{array}{c}\text { Chi- } \\
\text { Square }\end{array}$ & $\begin{array}{c}\text { P-Value } \\
\text { (Pr > ChiSq) }\end{array}$ \\
\hline StudentTeachRatio & 1 & 4.64 & 0.0312 \\
Region & 2 & 21.71 & $<.0001$ \\
SchoolStatus & 2 & 3.74 & 0.154 \\
SchoolLevel & 2 & 12.72 & 0.0017 \\
\hline
\end{tabular}


Table 5.12: Final Model for the Student-Teacher ratio effect (1996)

\begin{tabular}{|c|c|c|c|c|c|}
\hline \multicolumn{5}{|c|}{ Analysis Of GEE Parameter Estimates } & \multirow[b]{3}{*}{$\begin{array}{l}\text { Odds } \\
\text { Ratio }\end{array}$} \\
\hline \multicolumn{5}{|c|}{ Empirical Standard Error Estimates } & \\
\hline Parameter & Level & Estimate & $\begin{array}{l}\text { Standard } \\
\text { Error }\end{array}$ & $\begin{array}{l}\text { P-Value } \\
(\operatorname{Pr}>|Z|)\end{array}$ & \\
\hline Intercept & & -1.9371 & 0.2379 & $<.0001$ & \\
\hline StudentTeachRatio & & -0.0207 & 0.0108 & 0.0562 & 0.9795 \\
\hline Region & Apia Urban & -0.1783 & 0.1944 & 0.3591 & 0.8367 \\
\hline Region & Rest of Upolu & 0.5777 & 0.1403 & $<.0001$ & 1.7819 \\
\hline Region & Savaii & 0 & 0 & & 1.0000 \\
\hline SchoolLevel & Primary & -0.5114 & 0.141 & 0.0003 & 0.5997 \\
\hline SchoolLevel & Primary/Secondary & -0.2733 & 0.1678 & 0.1033 & 0.7609 \\
\hline SchoolLevel & Secondary & 0 & 0 & & 1.0000 \\
\hline
\end{tabular}

\begin{tabular}{|l|c|r|r|}
\hline \multicolumn{4}{|c|}{ Score Statistics For Type 3 GEE Analysis } \\
\hline \multicolumn{1}{|c|}{ Source } & DF & $\begin{array}{c}\text { Chi- } \\
\text { Square }\end{array}$ & $\begin{array}{c}\text { P-Value } \\
\text { (Pr > ChiSq) }\end{array}$ \\
\hline StudentTeachRatio & 1 & 4.34 & 0.0372 \\
Region & 2 & 22.65 & $<.0001$ \\
SchoolLevel & 2 & 9.99 & 0.0068 \\
\hline
\end{tabular}

In 1997, all the four covariates were significantly (p-values < 0.05) associated with the dropouts as shown by the Chi-Square statistics in Table 5.13. When keeping the effect of region, school status and school level effects constant, students were less likely to drop out of schools with higher student-teacher ratios just like the other calendar years. When controlling the effect of student-teacher ratio, school status and school level, students were more likely to drop out of schools in the Rest of Upolu region than those of the Savaii and Apia Urban regions. The odd of dropping out in the Rest of Upolu was $1.35(\mathrm{OR}=1.3526)$ times higher than that of Savaii region. But when holding the student-teacher ratio, region and school status effects fixed, students were less likely to drop out of Primary schools than Secondary schools. In other words, students were more likely to quit Secondary schools than Primary schools. The odd of quitting Secondary schools was $1.9(\mathrm{OR}=1.9022$, which is the inverse of 0.5257) times higher than that of Primary schools. 
Table 5.13: Final Model for the Student-Teacher ratio effect (1997)

\begin{tabular}{|c|c|c|c|c|c|}
\hline \multicolumn{5}{|c|}{ Analysis Of GEE Parameter Estimates } & \multirow[b]{3}{*}{$\begin{array}{l}\text { Odds } \\
\text { Ratio }\end{array}$} \\
\hline \multicolumn{5}{|c|}{ Empirical Standard Error Estimates } & \\
\hline Parameter & Level & Estimate & $\begin{array}{l}\text { Standard } \\
\text { Error }\end{array}$ & $\begin{array}{l}\text { P-Value } \\
(\operatorname{Pr}>|Z|)\end{array}$ & \\
\hline Intercept & & -1.1619 & 1.0235 & 0.2563 & \\
\hline StudentTeachRatio & & -0.0419 & 0.0109 & 0.0001 & 0.9590 \\
\hline Region & Apia Urban & -0.155 & 0.2084 & 0.4569 & 0.8564 \\
\hline Region & Rest of Upolu & 0.302 & 0.1487 & 0.0422 & 1.3526 \\
\hline Region & Savaii & 0 & 0 & & 1.0000 \\
\hline SchoolStatus & Government & -0.0982 & 1.0541 & 0.9258 & 0.9065 \\
\hline SchoolStatus & Mission & -0.644 & 1.066 & 0.5458 & 0.5252 \\
\hline SchoolStatus & Private & 0 & 0 & & 1.0000 \\
\hline SchoolLevel & Primary & -0.643 & 0.1496 & $<.0001$ & 0.5257 \\
\hline SchoolLevel & Primary/Secondary & 0.1892 & 0.1824 & 0.2995 & 1.2083 \\
\hline SchoolLevel & Secondary & 0 & 0 & & 1.0000 \\
\hline
\end{tabular}

\begin{tabular}{|l|r|rr|}
\hline \multicolumn{4}{|c|}{ Score Statistics For Type 3 GEE Analysis } \\
\hline \multicolumn{1}{|c|}{ Source } & DF & $\begin{array}{c}\text { Chi- } \\
\text { Square }\end{array}$ & $\begin{array}{c}\text { P-Value } \\
\text { (Pr > ChiSq) }\end{array}$ \\
\hline StudentTeachRatio & 1 & 10.55 & 0.0012 \\
Region & 2 & 10.12 & 0.0063 \\
SchoolStatus & 2 & 7.09 & 0.0288 \\
SchoolLevel & 2 & 12.27 & 0.0022 \\
\hline
\end{tabular}

But in 1998, 2002 and 2003 only the student-teacher ratio was significantly (p-value < 0.05) associated with the dropouts. The final models for these calendar years are found in Appendix A. The models show that students were less likely to drop out of schools with higher student-teacher ratios. However, in 1999 the school status coupled with student-teacher ratio was significantly (p-values $<0.05)$ associated with the response variable as displayed by the Chi-Square statistics in Table 5.14. The final model for 1999 is presented in Table 5.15 whereby students were more likely to drop out of Government schools as compared to Mission and Private schools. 


\begin{tabular}{|l|r|r|r|}
\hline \multicolumn{4}{|c|}{ Score Statistics For Type 3 GEE Analysis } \\
\hline \multicolumn{1}{|c|}{ Source } & DF & $\begin{array}{c}\text { Chi- } \\
\text { Square }\end{array}$ & $\begin{array}{c}\text { P-Value } \\
\text { (Pr > ChiSq) }\end{array}$ \\
\hline StudentTeachRatio & 1 & 13.67 & 0.0002 \\
Region & 2 & 5.06 & 0.0796 \\
SchoolStatus & 2 & 9.01 & 0.011 \\
SchoolLevel & 2 & 5.12 & 0.0773 \\
\hline
\end{tabular}

Table 5.15: Final Model for the Student-Teacher ratio effect (1999)

\begin{tabular}{|c|c|c|c|c|c|}
\hline \multicolumn{5}{|c|}{ Analysis Of GEE Parameter Estimates } & \\
\hline \multicolumn{6}{|c|}{ Empirical Standard Error Estimates } \\
\hline Parameter & Level & Estimate & $\begin{array}{l}\text { Standard } \\
\text { Error }\end{array}$ & $\begin{array}{l}\text { P-Value } \\
(\operatorname{Pr}>|Z|)\end{array}$ & $\begin{array}{l}\text { Odds } \\
\text { Ratio }\end{array}$ \\
\hline Intercept & & -1.9904 & 0.7764 & 0.0104 & \\
\hline StudentTeachRatio & & -0.0644 & 0.0118 & $<.0001$ & 0.9376 \\
\hline SchoolStatus & Government & 0.8776 & 0.7683 & 0.2534 & 2.4051 \\
\hline SchoolStatus & Mission & -0.0434 & 0.8019 & 0.9569 & 0.9575 \\
\hline SchoolStatus & Private & 0 & 0 & & 1.0000 \\
\hline
\end{tabular}

\begin{tabular}{|l|c|r|r|}
\hline \multicolumn{4}{|c|}{ Score Statistics For Type 3 GEE Analysis } \\
\hline \multicolumn{1}{|c|}{ Source } & DF & $\begin{array}{c}\text { Chi- } \\
\text { Square }\end{array}$ & $\begin{array}{c}\text { P-Value } \\
(\mathbf{P r}>\text { ChiSq) }\end{array}$ \\
\hline StudentTeachRatio & 1 & 16.11 & $<.0001$ \\
SchoolStatus & 2 & 11.38 & 0.0034 \\
\hline
\end{tabular}

In 2000, dropping out of school was again associated with the student-teacher ratio and region based on the Chi-Square statistics in Table 5.16. The final model for this calendar year is found in Table 5.17 when school status and school level were removed from the model one at a time due to their insignicance ( $\mathrm{p}$-values $>0.05$ ). The model shows that holding the region effect constant; students were less likely to drop out of schools with higher student-teacher ratios. On the other hand, when the studentteacher effect is fixed, students were more likely to withdraw from schools in the Rest of Upolu than those of Savaii and Apia Urban regions. The odd of dropping out in the Rest of Upolu was $1.56(\mathrm{OR}=1.5580)$ times higher than that of Savaii. The same trend was also seen in 2001. However, the odd of dropping out of school in the Rest of Upolu region was $1.48(\mathrm{OR}=1.4783)$ times higher than that of the Savaii region as shown in Table 5.19. For 2001, only the student-teacher ratio and region were 
significantly ( $\mathrm{p}$-values $<0.05$ ) associated with the dropouts based on the Chi-Square statistics in Table 5.18.

Table 5.16: Chi-Square Statistics for the Model with four Co-variates (2000)

\begin{tabular}{|l|r|r|r|}
\hline \multicolumn{4}{|c|}{ Score Statistics For Type 3 GEE Analysis } \\
\hline \multicolumn{1}{|c|}{ Source } & DF & $\begin{array}{c}\text { Chi- } \\
\text { Square }\end{array}$ & $\begin{array}{c}\text { P-Value } \\
\text { (Pr > ChiSq) }\end{array}$ \\
\hline StudentTeachRatio & 1 & 9 & 0.0027 \\
Region & 2 & 7.83 & 0.02 \\
SchoolStatus & 2 & 4.71 & 0.0949 \\
SchoolLevel & 2 & 4.53 & 0.1038 \\
\hline
\end{tabular}

Table 5.17: Final Model for the Student-Teacher ratio effect (2000)

\begin{tabular}{|c|c|c|c|c|c|}
\hline \multicolumn{5}{|c|}{ Analysis Of GEE Parameter Estimates } & \\
\hline \multicolumn{6}{|c|}{ Empirical Standard Error Estimates } \\
\hline Parameter & Level & Estimate & $\begin{array}{l}\text { Standard } \\
\text { Error }\end{array}$ & $\begin{array}{c}\text { P-Value } \\
(\mathrm{Pr}>|\mathrm{Z}|)\end{array}$ & $\begin{array}{l}\text { Odds } \\
\text { Ratio }\end{array}$ \\
\hline Intercept & & -1.8697 & 0.2217 & $<.0001$ & \\
\hline StudentTeachRatio & & -0.0386 & 0.0098 & $<.0001$ & 0.9621 \\
\hline Region & Apia Urban & 0.3134 & 0.1975 & 0.1125 & 1.3681 \\
\hline Region & Rest of Upolu & 0.4434 & 0.1593 & 0.0054 & 1.5580 \\
\hline Region & Savaii & 0 & 0 & & 1.0000 \\
\hline
\end{tabular}

\begin{tabular}{|l|r|r|r|}
\hline \multicolumn{4}{|c|}{ Score Statistics For Type 3 GEE Analysis } \\
\hline \multicolumn{1}{|c|}{ Source } & DF & \multicolumn{1}{c|}{$\begin{array}{c}\text { Chi- } \\
\text { Square }\end{array}$} & $\begin{array}{c}\text { P-Value } \\
\text { (Pr > ChiSq) }\end{array}$ \\
\hline StudentTeachRatio & 1 & 10.36 & 0.0013 \\
Region & 2 & 6.56 & 0.0375 \\
\hline
\end{tabular}

Table 5.18: Chi-Square Statistics for the Model with four Co-variates (2001)

\begin{tabular}{|l|r|r|r|}
\hline \multicolumn{4}{|c|}{ Score Statistics For Type 3 GEE Analysis } \\
\hline \multicolumn{1}{|c|}{ Source } & DF & $\begin{array}{c}\text { Chi- } \\
\text { Square }\end{array}$ & $\begin{array}{c}\text { P-Value } \\
\text { (Pr > ChiSq) }\end{array}$ \\
\hline StudentTeachRatio & 1 & 9.21 & 0.0024 \\
Region & 2 & 7.91 & 0.0192 \\
SchoolStatus & 2 & 2.94 & 0.2295 \\
SchoolLevel & 2 & 5.32 & 0.07 \\
\hline
\end{tabular}


Table 5.19: Final Model for the Student-Teacher ratio effect (2001)

\begin{tabular}{|c|c|c|c|c|c|}
\hline \multicolumn{5}{|c|}{ Analysis Of GEE Parameter Estimates } & \multirow[b]{3}{*}{$\begin{array}{l}\text { Odds } \\
\text { Ratio }\end{array}$} \\
\hline \multicolumn{5}{|c|}{ Empirical Standard Error Estimates } & \\
\hline Parameter & Level & Estimate & $\begin{array}{l}\text { Standard } \\
\text { Error }\end{array}$ & $\begin{array}{c}\text { P-Value } \\
(\operatorname{Pr}>|Z|)\end{array}$ & \\
\hline Intercept & & -1.9279 & 0.2324 & $<.0001$ & \\
\hline StudentTeachRatio & & -0.0389 & 0.0093 & $<.0001$ & 0.9618 \\
\hline Region & Apia Urban & 0.1054 & 0.1959 & 0.5904 & 1.1112 \\
\hline Region & Rest of Upolu & 0.3909 & 0.1545 & 0.0114 & 1.4783 \\
\hline Region & Savaii & 0 & 0 & & 1.0000 \\
\hline
\end{tabular}

\begin{tabular}{|l|c|c|c|}
\hline \multicolumn{4}{|c|}{ Score Statistics For Type 3 GEE Analysis } \\
\hline \multicolumn{1}{|c|}{ Source } & DF & $\begin{array}{c}\text { Chi- } \\
\text { Square }\end{array}$ & $\begin{array}{c}\text { P-Value } \\
(\text { Pr > ChiSq) }\end{array}$ \\
\hline StudentTeachRatio & 1 & 15.6 & $<.0001$ \\
Region & 2 & 6.74 & 0.0344 \\
\hline
\end{tabular}

For 2002 and 2003 we assume that the student-teacher ratio was correlated with the three categorical explanatory variables, region, school status and school level. We have already shown in Figure 5.1 and Figure 5.2 that fewer dropouts were found in the Apia Urban region in which it has higher student-teacher ratios.

For the same reason we could argue that for the school status effect in section 5.3 we have found that students were less likely to drop out of Private schools than in Mission and Government. Clearly, higher student-teacher ratios were found in Private school as compared to those of the Mission as shown by Figure 5.3 and Figure 5.5. Similarly for school level effect, we also found in section 5.2 that students were less likely to drop out in Primary than Secondary. Yet, student-teacher ratios for Primary schools were much higher than Secondary and Primary/Secondary schools as shown by Figure 5.4 and Figure 5.6. 
COMPARING THE STUDENT-TEACHER RATIO

BY SCHOOL STATUS (2002)

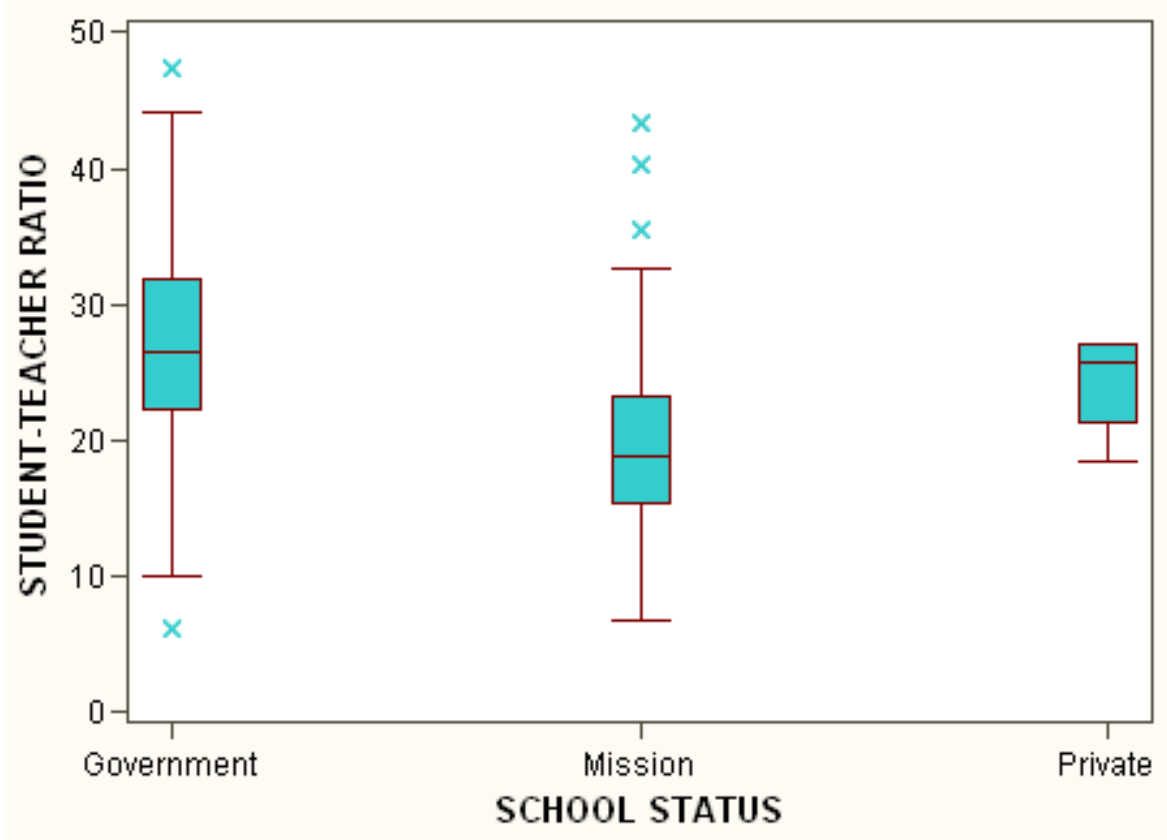

Figure 5.3: Distribution of the Student-Teacher Ratio by School Status (2002)

COMPARING THE STUDENT-TEACHER RATIO

BY SCHOOL LEVEL (2002)

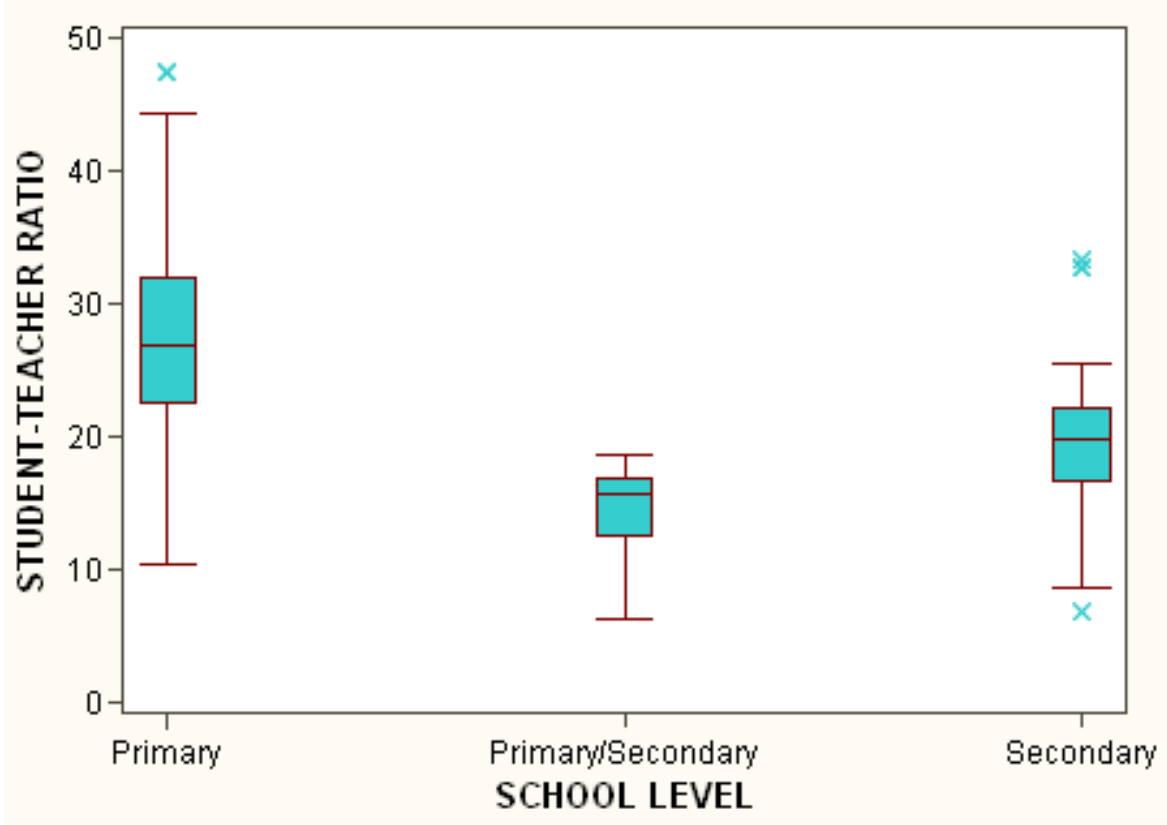

Figure 5.4: Distribution of the Student-Teacher Ratio by School Level (2002) 
COMPARING THE STUDENT-TEACHER RATIO

BY SCHOOL STATUS (2003)

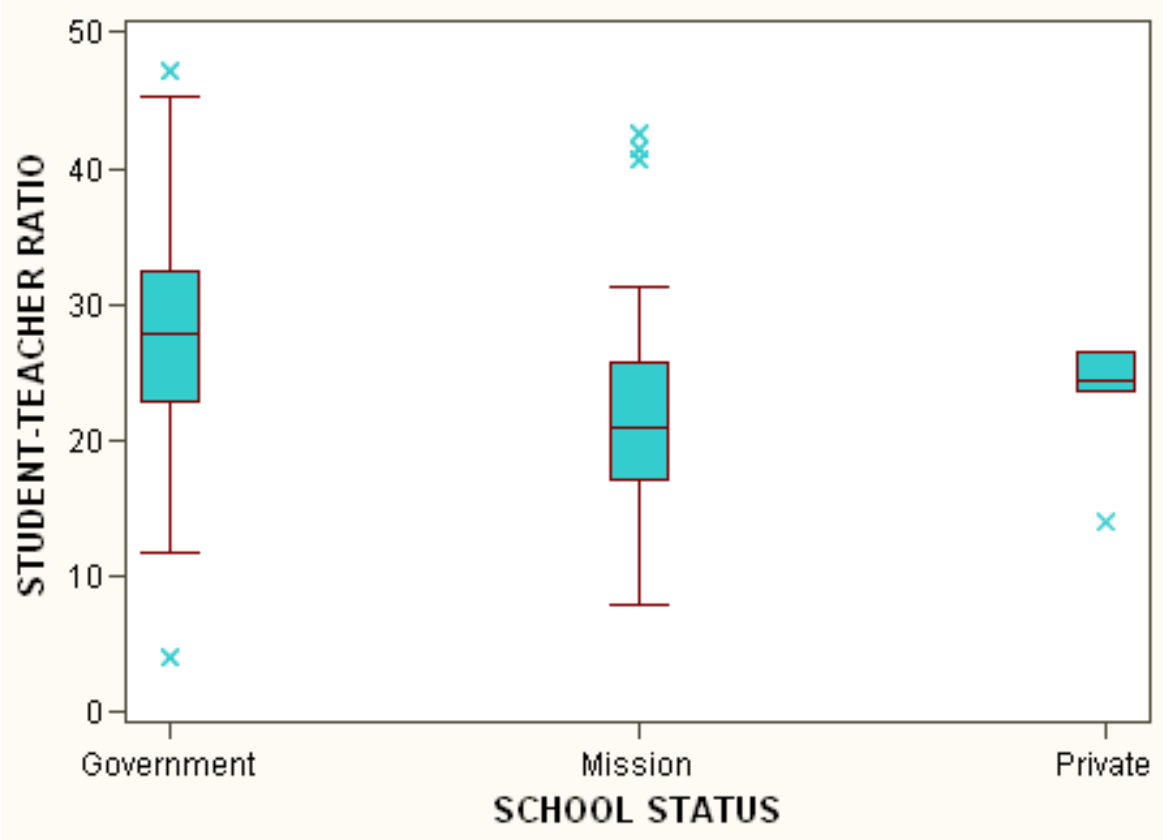

Figure 5.5: Distribution of the Student-Teacher Ratio by School Status (2003)

COMPARING THE STUDENT-TEACHER RATIO

BY SCHOOL LEVEL (2003)

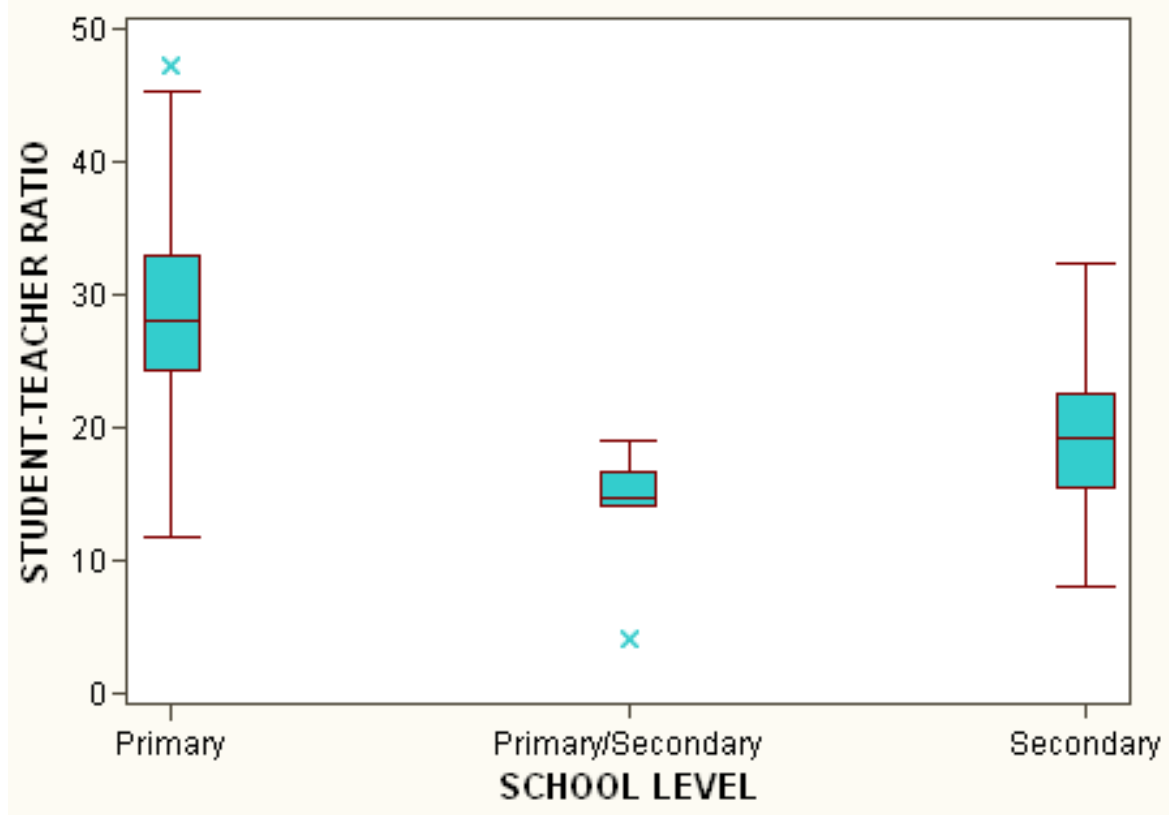

Figure 5.6: Distribution of the Student-Teacher Ratio by School Level (2003) 
When we fitted the model for 2004, the school status was obviously insignificant as well as the school level according to the Chi-Square statistics in Table 5.20. However, when the school status was removed all three covariates, the student-teacher ratio, region and school level were significantly (p-values $<0.05$ ) associated with the dropouts as presented in Table 5.21. From this final model for this calendar year, when holding the region and school level effects constant, students were less likely to drop out of schools with higher student-teacher ratios. When keeping the studentteacher ratio and school level effects fixed, students were more likely to leave schools prematurely in the Rest of Upolu than those in the Savaii region. The odd of dropping out in the Rest of Upolu was $1.57(\mathrm{OR}=1.5724)$ times higher than that of Savaii. But when both student-teacher ratio and region were held fixed, students were more likely to drop out of Secondary schools than the Primary and Primary/Secondary. The odds of dropping out in the Secondary school as compared to that of Primary and Primary/Secondary were $1.38(\mathrm{OR}=1.3801$, which is the inverse of 0.7246$)$ and 8.94 $(\mathrm{OR}=8.9445$, which is the inverse of 0.1118$)$ times higher, respectively. More insight into having fewer dropouts in the Primary schools than in the Secondary schools is graphically displayed by Figure 5.7. With Primary schools having higher studentteacher ratios, thus fewer dropouts.

Table 5.20: Chi-Square Statistics for the Model with four Co-variates (2004)

\begin{tabular}{|l|r|r|r|}
\hline \multicolumn{4}{|c|}{ Score Statistics For Type 3 GEE Analysis } \\
\hline \multicolumn{1}{|c|}{ Source } & DF & \multicolumn{1}{c|}{$\begin{array}{c}\text { Chi- } \\
\text { Square }\end{array}$} & $\begin{array}{c}\text { P-Value } \\
\text { (Pr > ChiSq) }\end{array}$ \\
\hline StudentTeachRatio & 1 & 9.11 & 0.0025 \\
Region & 2 & 7.86 & 0.0197 \\
SchoolStatus & 2 & 1.5 & 0.4728 \\
SchoolLevel & 2 & 4.96 & 0.0837 \\
\hline
\end{tabular}


Table 5.21: Final Model for the Student-Teacher ratio effect (2004)

\begin{tabular}{|c|c|c|c|c|c|}
\hline \multicolumn{5}{|c|}{ Analysis Of GEE Parameter Estimates } & \multirow[b]{3}{*}{$\begin{array}{l}\text { Odds } \\
\text { Ratio }\end{array}$} \\
\hline \multicolumn{5}{|c|}{ Empirical Standard Error Estimates } & \\
\hline Parameter & Level & Estimate & $\begin{array}{l}\text { Standard } \\
\text { Error }\end{array}$ & $\begin{array}{l}\text { P-Value } \\
(\operatorname{Pr}>|Z|)\end{array}$ & \\
\hline Intercept & & -1.772 & 0.2633 & $<.0001$ & \\
\hline StudentTeachRatio & & -0.0359 & 0.011 & 0.0011 & 0.9647 \\
\hline Region & Apia Urban & 0.0879 & 0.229 & 0.7009 & 1.0919 \\
\hline Region & Rest of Upolu & 0.4526 & 0.1623 & 0.0053 & 1.5724 \\
\hline Region & Savaii & 0 & 0 & & 1.0000 \\
\hline SchoolLevel & Primary & -0.3221 & 0.1589 & 0.0426 & 0.7246 \\
\hline SchoolLevel & Primary/Secondary & -2.1914 & 0.651 & 0.0008 & 0.1118 \\
\hline SchoolLevel & Secondary & 0 & 0 & & 1.0000 \\
\hline
\end{tabular}

\begin{tabular}{|l|c|c|c|}
\hline \multicolumn{4}{|c|}{ Score Statistics For Type 3 GEE Analysis } \\
\hline \multicolumn{1}{|c|}{ Source } & DF & $\begin{array}{c}\text { Chi- } \\
\text { Square }\end{array}$ & $\begin{array}{c}\text { P-Value } \\
\text { (Pr > ChiSq) }\end{array}$ \\
\hline StudentTeachRatio & 1 & 9.38 & 0.0022 \\
Region & 2 & 8.38 & 0.0151 \\
SchoolLevel & 2 & 6.04 & 0.0487 \\
\hline
\end{tabular}

\section{COMPARING THE STUDENT-TEACHER RATIO BY SCHOOL LEVEL (2004)}

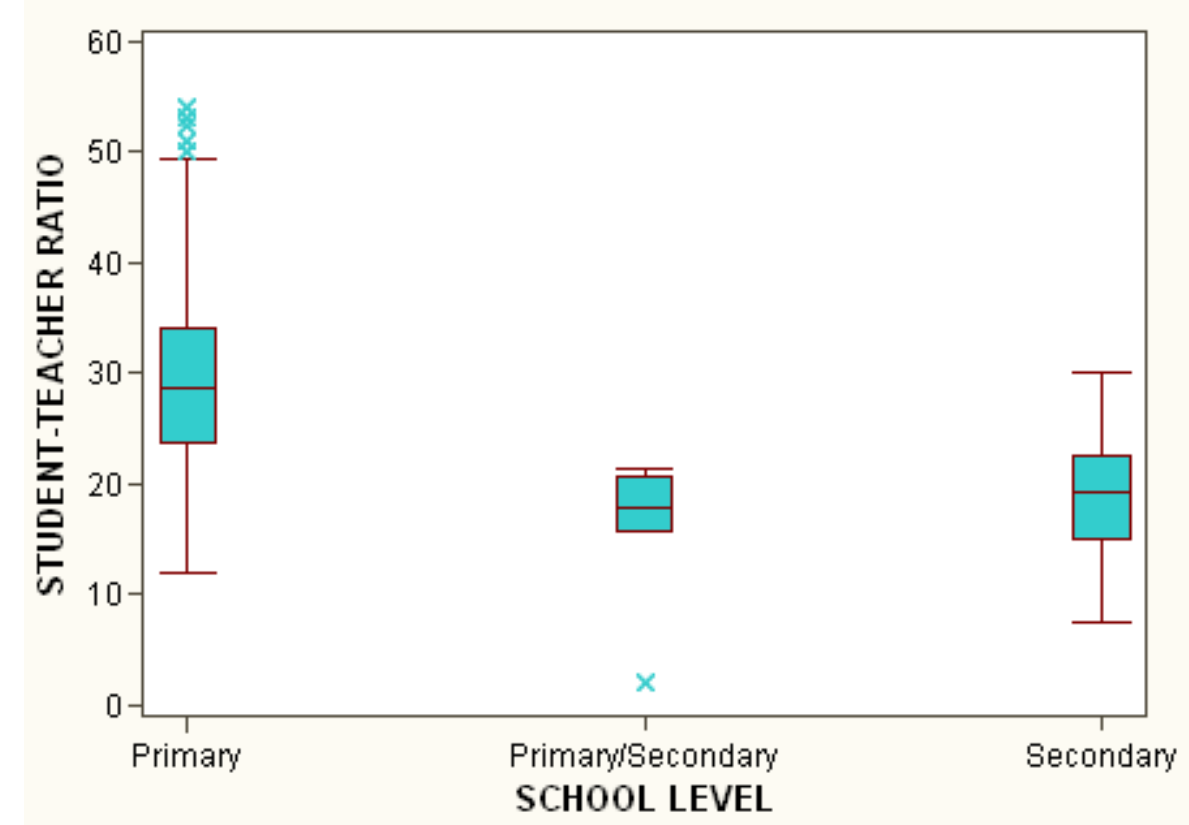

Figure 5.7: Distribution of the Student-Teacher Ratio by School Level (2004) 
For the year 2005, only student-teacher ratio and region covariates were significantly associated with the dropouts based on the Chi-Square statistics in Table 5.22. The other two covariates were insignificant therefore removed from the model one at a time. According to the final model presented in Table 5.23 students were less likely to drop out of schools with higher student-teacher ratios while keeping the region effect fixed. But when the student-teacher ratio effect is being held constant, students were more likely to drop out of schools in both the Apia Urban and Rest of Upolu regions. The odds of giving up school in the Apia Urban and Rest of Upolu regions were 1.58 $(\mathrm{OR}=1.5839)$ and $1.56(\mathrm{OR}=1.5566)$ times higher than that of the Savaii region respectively. Given school status and school level were not significant but we suspected that school status and school level are correlated and simultaneously, school level and student-teacher ratio are also correleated. For this reason both covariates have been dropped from the model. Figure 5.8 and Figure 5.9 portray this intercorrelation.

Table 5.22: Chi-Square Statistics for the Model with four Co-variates (2005)

\begin{tabular}{|l|r|r|r|}
\hline \multicolumn{4}{|c|}{ Score Statistics For Type 3 GEE Analysis } \\
\hline \multicolumn{1}{|c|}{ Source } & DF & $\begin{array}{c}\text { Chi- } \\
\text { Square }\end{array}$ & $\begin{array}{c}\text { P-Value } \\
\text { (Pr > ChiSq) }\end{array}$ \\
\hline StudentTeachRatio & 1 & 6.16 & 0.0131 \\
Region & 2 & 6.7 & 0.035 \\
SchoolStatus & 2 & 0.1 & 0.9511 \\
SchoolLevel & 2 & 1.38 & 0.5015 \\
\hline
\end{tabular}

Table 5.23: Final Model for the Student-Teacher ratio effect (2005)

\begin{tabular}{|c|c|c|c|c|c|}
\hline \multicolumn{5}{|c|}{ Analysis Of GEE Parameter Estimates } & \multirow[b]{3}{*}{$\begin{array}{l}\text { Odds } \\
\text { Ratio }\end{array}$} \\
\hline \multicolumn{5}{|c|}{ Empirical Standard Error Estimates } & \\
\hline Parameter & Level & Estimate & $\begin{array}{l}\text { Standard } \\
\text { Error }\end{array}$ & $\begin{array}{l}\text { P-Value } \\
(\operatorname{Pr}>|Z|)\end{array}$ & \\
\hline Intercept & & -2.0588 & 0.2752 & $<.0001$ & \\
\hline StudentTeachRatio & & -0.0335 & 0.0092 & 0.0003 & 0.9671 \\
\hline Region & Apia Urban & 0.4599 & 0.2104 & 0.0288 & 1.5839 \\
\hline Region & Rest of Upolu & 0.4425 & 0.1622 & 0.0064 & 1.5566 \\
\hline Region & Savaii & 0 & 0 & & 1.0000 \\
\hline
\end{tabular}

\begin{tabular}{|l|r|r|r|}
\hline \multicolumn{4}{|c|}{ Score Statistics For Type 3 GEE Analysis } \\
\hline \multicolumn{1}{|c|}{ Source } & DF & $\begin{array}{c}\text { Chi- } \\
\text { Square }\end{array}$ & $\begin{array}{c}\text { P-Value } \\
\text { (Pr > ChiSq) }\end{array}$ \\
\hline StudentTeachRatio & 1 & 9.5 & 0.0021 \\
Region & 2 & 7.48 & 0.0238 \\
\hline
\end{tabular}


COMPARING THE STUDENT-TEACHER RATIO

BY SCHOOL STATUS (2005)

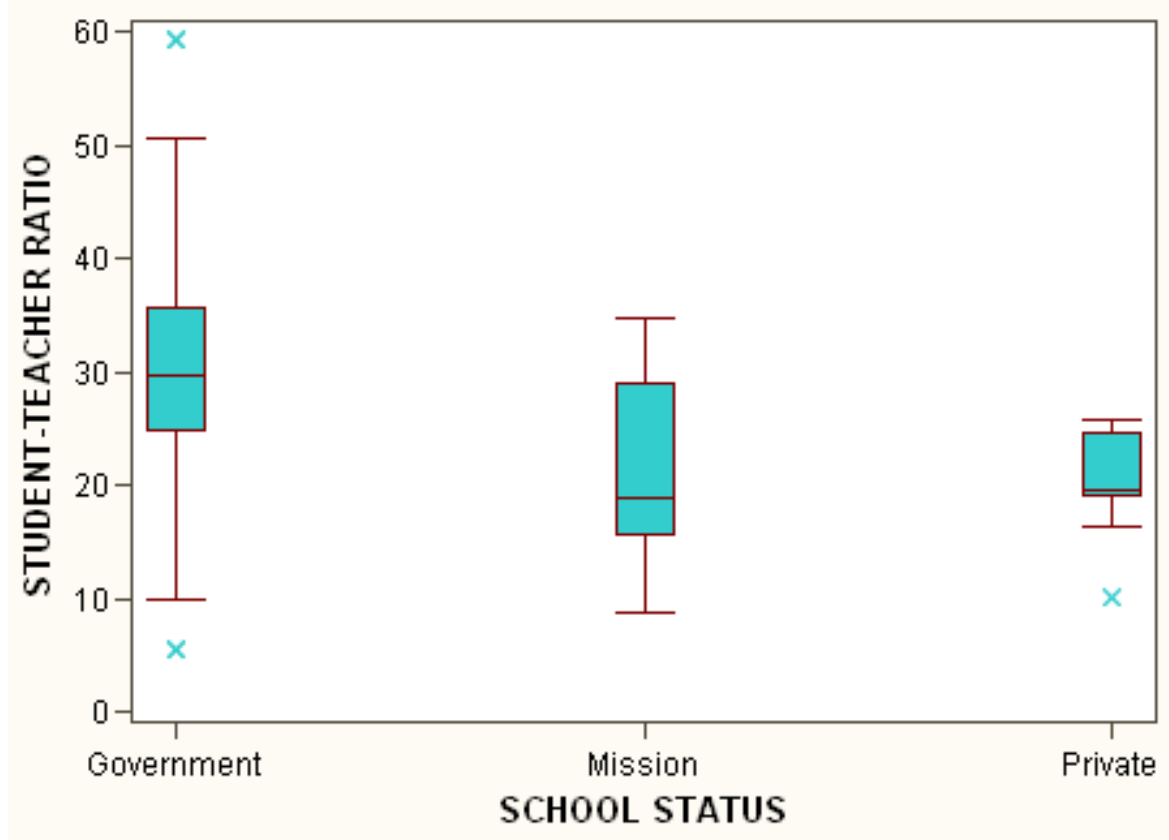

Figure 5.8: Distribution of the Student-Teacher Ratio by School Status (2005)

COMPARING THE STUDENT-TEACHER RATIO

BY SCHOOL LEVEL (2005)

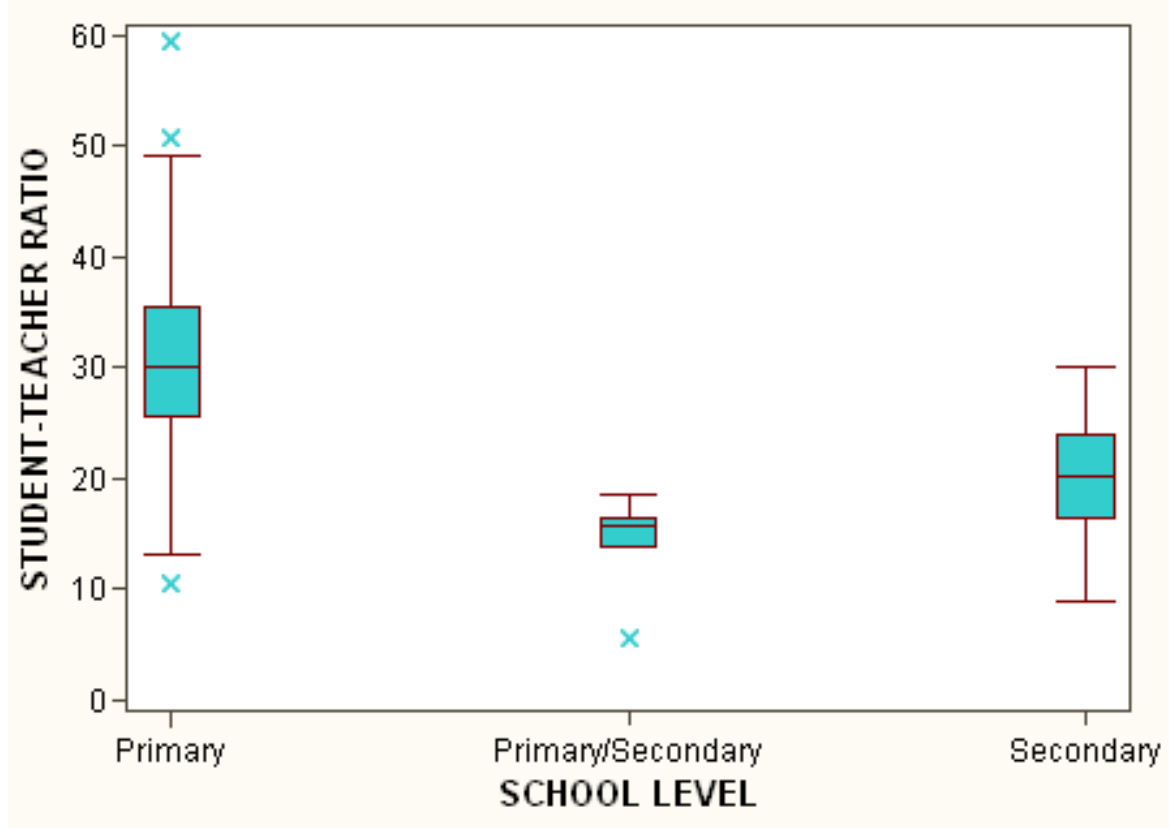

Figure 5.9: Distribution of the Student-Teacher Ratio by School Level (2005) 
The year 2006 saw the school level covariate insignificantly associated with the dropout as shown by the Chi-Square statistics in Table 5.24. This could be assumed that the school level covariate was correlated with the other explanatory variable, the student-teacher ratio. This correlation could be explained by Figure 5.10, whereby higher student-teacher ratios were found in the Primary schools in which students were less likely to drop out of.

Despite this correlation, but when the school level has been removed from the model, all the remaining three covariates, student-teacher ratio, region and school status, were significantly ( $\mathrm{p}$-values $<0.05)$ associated with the dropout. The final model is presented in Table 5.25. It shows the same story as in the previous calendar years, whereby students were less likely from schools with higher student-teacher ratios when the region and school status effects were kept constant. Further, by holding the student-teacher ratio and school status effects constant, students were more likely to quit schools in the Rest of Upolu region than those in the Savaii region. The odds of dropping out in the Rest of Upolu was $1.61(\mathrm{OR}=1.6064)$ times higher than that of Savaii. But by keeping the student-teacher ratio and region effects fixed, students were more likely to drop out of Government schools as compared to those of the Private schools. The odds of dropping out was $1.77(\mathrm{OR}=1.7674)$ times higher.

Table 5.24: Chi-Square Statistics for the Model with four Co-variates (2006)

\begin{tabular}{|l|r|r|r|}
\hline \multicolumn{4}{|c|}{ Score Statistics For Type 3 GEE Analysis } \\
\hline \multicolumn{1}{|c|}{ Source } & DF & \multicolumn{1}{c|}{$\begin{array}{c}\text { Chi- } \\
\text { Square }\end{array}$} & $\begin{array}{c}\text { P-Value } \\
\text { (Pr > ChiSq) }\end{array}$ \\
\hline StudentTeachRatio & 1 & 24.6 & $<.0001$ \\
Region & 2 & 11.47 & 0.0032 \\
SchoolStatus & 2 & 7.36 & 0.0253 \\
SchoolLevel & 2 & 3.37 & 0.1859 \\
\hline
\end{tabular}


Table 5.25: Final Model for the Student-Teacher ratio effect (2006)

\begin{tabular}{|c|c|c|c|c|c|}
\hline \multicolumn{5}{|c|}{ Analysis Of GEE Parameter Estimates } & \multirow[b]{3}{*}{$\begin{array}{l}\text { Odds } \\
\text { Ratio }\end{array}$} \\
\hline \multicolumn{5}{|c|}{ Empirical Standard Error Estimates } & \\
\hline Parameter & Level & Estimate & $\begin{array}{l}\text { Standard } \\
\text { Error }\end{array}$ & $\begin{array}{c}\text { P-Value } \\
(\operatorname{Pr}>|Z|)\end{array}$ & \\
\hline Intercept & & -2.1022 & 0.3212 & $<.0001$ & \\
\hline StudentTeachRatio & & -0.0428 & 0.0095 & $<.0001$ & 0.9581 \\
\hline Region & Apia Urban & 0.2305 & 0.1488 & 0.1214 & 1.2592 \\
\hline Region & Rest of Upolu & 0.474 & 0.1251 & 0.0002 & 1.6064 \\
\hline Region & Savaii & 0 & 0 & & 1.0000 \\
\hline SchoolStatus & Government & 0.5695 & 0.2627 & 0.0301 & 1.7674 \\
\hline SchoolStatus & Mission & -0.0101 & 0.3144 & 0.9744 & 0.9900 \\
\hline SchoolStatus & Private & 0 & 0 & & 1.0000 \\
\hline
\end{tabular}

\begin{tabular}{|l|c|r|r|}
\hline \multicolumn{4}{|c|}{ Score Statistics For Type 3 GEE Analysis } \\
\hline \multicolumn{1}{|c|}{ Source } & DF & $\begin{array}{c}\text { Chi- } \\
\text { Square }\end{array}$ & $\begin{array}{c}\text { P-Value } \\
(\text { Pr > ChiSq) }\end{array}$ \\
\hline StudentTeachRatio & 1 & 22.56 & $<.0001$ \\
Region & 2 & 12.35 & 0.0021 \\
SchoolStatus & 2 & 9.71 & 0.0078 \\
\hline
\end{tabular}

COMPARING THE STUDENT-TEACHER RATIO

BY SCHOOL LEVEL (2006)

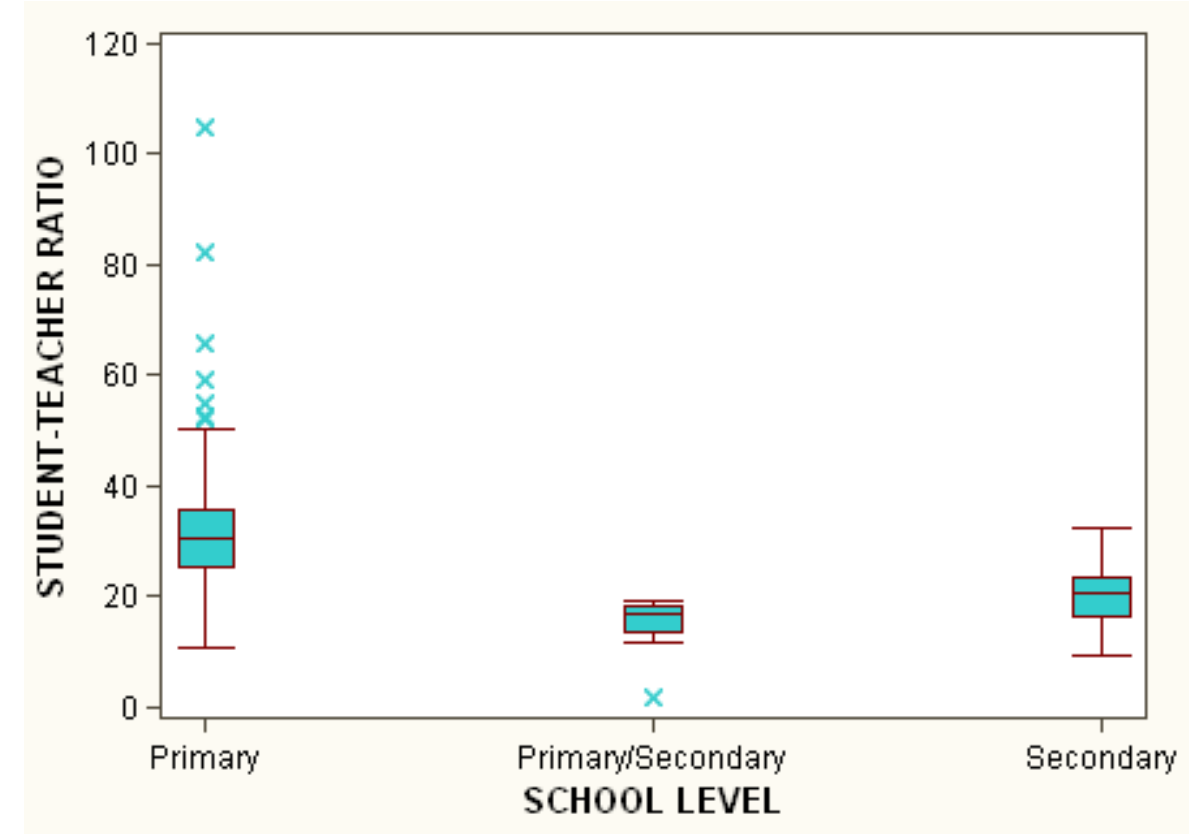

Figure 5.10: Distribution of the Student-Teacher Ratio by School Level (2006) 


\subsection{The School Size (Total Enrolment) Effect.}

As mentioned in the previous section, 5.4 that the effect of the covariate, school size (total enrolment) on the response variable will be modelled separately due to its association with the covariate, student-teacher ratio. From the results it was very apparent that higher student-teacher ratios were caused by larger number of enrolments or larger schools.

Many educational studies found that the explanatory variable, school size (total enrolment) is one of the significant predictors in predicting the dropout. Statistically, it has been commonly found that dropping out of school regardless the school level, goes with the school size. That is more students are likely to leave school prematurely from larger schools than smaller ones. Some researchers assumed that students from smaller schools get much attention and assistance from the teacher(s) as compared to larger schools. Others argue that there are other within schools and social and random factors that need to be considered on why larger schools have more dropouts than smaller ones.

For this study we also found that school size (total enrolment) was significantly (pvalue < 0.05) associated with the dropout based on the Score Statistics, for some of the calendar years. This association was seen in 1995, 1996, 1997, 1998, 1999 and 2006. The school size was not significant for other years but the models will be found in Appendix A.

\section{Model Interpretation}

The study found that school size (total enrolment) was adversely (negative estimates) associated with the response variable, dropout. That is, students were less likely to drop out of larger schools in 1995, 1996, 1997, 1998, 1999 and 2006. The models for 1995 and 2006 are presented in Table 5.26 and Table 5.27 respectively, while the models for 1996, 1997, 1998 and 1999 are found in Appendix A. The results are quite similar to those of the student-teacher ratio in section 5.4. This is contrary to most of the findings in the literature review section (chapter 2). However, evidence was already presented in section 5.4 as why fewer dropouts were found in larger schools in Samoa. 
Table 5.26: The Model for the School Size (Total Enrolment) effect (1995)

\begin{tabular}{|c|c|c|c|c|}
\hline \multicolumn{4}{|c|}{ Analysis Of GEE Parameter Estimates } & \multirow{2}{*}{} \\
\cline { 1 - 3 } Parameter & Estimate & $\begin{array}{c}\text { Standard } \\
\text { Error }\end{array}$ & $\begin{array}{c}\text { P-Value } \\
\text { (Pr }>|\mathbf{Z}| \mathbf{)}\end{array}$ & $\begin{array}{c}\text { Odd } \\
\text { Ratio }\end{array}$ \\
\hline Intercept & -2.6656 & 0.1641 & $<.0001$ & \\
TotEnrol & -0.0008 & 0.0005 & 0.1158 & 0.9992 \\
\hline
\end{tabular}

\begin{tabular}{|c|c|c|c|}
\hline \multicolumn{4}{|c|}{ Score Statistics For Type 3 GEE Analysis } \\
\hline Source & DF & $\begin{array}{c}\text { Chi- } \\
\text { Square }\end{array}$ & $\begin{array}{c}\text { P-Value } \\
(\mathrm{Pr}>\mathrm{ChiSq})\end{array}$ \\
\hline & & 5.06 & 0.0245 \\
\hline
\end{tabular}

Table 5.27: The Model for the School Size (Total Enrolment) effect (2006)

\begin{tabular}{|c|c|c|c|c|}
\hline \multicolumn{4}{|c|}{ Analysis Of GEE Parameter Estimates } & \\
\hline \multicolumn{4}{|c|}{ Empirical Standard Error Estimates } & \\
\hline Parameter & Estimate & $\begin{array}{c}\text { Standard } \\
\text { Error }\end{array}$ & $\begin{array}{l}\text { P-Value } \\
(\operatorname{Pr}>|Z|)\end{array}$ & $\begin{array}{l}\text { Odd } \\
\text { Ratio }\end{array}$ \\
\hline Intercept & -2.2963 & 0.1622 & $<.0001$ & \\
\hline TotEnrol & -0.0009 & 0.0004 & 0.0521 & 0.9991 \\
\hline
\end{tabular}

\begin{tabular}{|c|c|c|c|}
\hline \multicolumn{4}{|c|}{ Score Statistics For Type 3 GEE Analysis } \\
\hline Source & DF & $\begin{array}{c}\text { Chi- } \\
\text { Square }\end{array}$ & $\begin{array}{c}\text { P-Value } \\
\text { (Pr }>\text { ChiSq) }\end{array}$ \\
\hline TotEnrol & 1 & 11.12 & 0.0009 \\
\hline
\end{tabular}

\subsection{The Secondary School Teacher Variables Effect (2007)}

This section reports findings on school teacher variables that associate with the dropout. The study wishes to investigate whether the teacher's gender, which is measured by the proportion of female teachers (PropFemales), has an impact on dropout. In addition we would also like to find out whether the ethnicity of school teachers, which is measured by the proportion of Samoan teachers (PropSamoans), is 
associated with the response variable. Finally, the study also wants to find out whether the school teacher's qualification, which is measured by those with teaching Certificates and those with Degrees, has an impact on the dropout. It is important to note here that some teachers only have the teaching certificate or the degree, while some have both. We only use the information on these variables from the 2007 survey as it was the most recent survey being carried out. Information on the same variables for other years (1995 - 2006) was not available.

\section{Model Interpretation}

According to the Chi-Square statistics shown in Table 5.28 (see full model in Appendix B), the teacher's gender has nothing to do with students who dropped out of school. When the teacher's gender (PropFemales) is being removed from the model, the Chi-Square statistics in Table 5.29 shows that only the teacher's ethnicity and qualification (Degrees) associate with the dropout. It appears that students are less likely to give up school early when there are more Samoan teachers in the school. In other words, students are $36.6(\mathrm{OR}=36.63$ which is the inverse of 0.0273$)$ times more likely to drop out of schools that have fewer Samoan teachers. Similarly for schools that have highly qualified teachers; that is students are less likely to leave school prematurely when there are more teachers with degrees in the school. Alternatively, students are $1.03(\mathrm{OR}=1.0348)$ times more likely to drop out of schools that have more teachers with just the Certificates.

Table 5.28: The Chi-Square Statistics of Full Model for 2007 Secondary School Teacher Variables

\begin{tabular}{|l|c|c|c|}
\hline \multicolumn{4}{|c|}{ Score Statistics For Type 3 GEE Analysis } \\
\hline Source & DF & $\begin{array}{c}\text { Chi- } \\
\text { Square }\end{array}$ & $\begin{array}{c}\text { P-Value } \\
(\mathbf{P r}>\text { ChiSq) }\end{array}$ \\
\hline PropFemales & 1 & 0.45 & 0.5042 \\
PropSamoans & 1 & 4.67 & 0.0308 \\
Certificates & 1 & 3.24 & 0.0717 \\
Degrees & 1 & 6.16 & 0.0131 \\
\hline
\end{tabular}


Table 5.29: The Simpler Model for 2007 Secondary School Teacher Variables

\begin{tabular}{|c|c|c|c|c|}
\hline \multicolumn{4}{|c|}{ Analysis Of GEE Parameter Estimates } & \multirow[b]{3}{*}{$\begin{array}{l}\text { Odds } \\
\text { Ratio }\end{array}$} \\
\hline \multicolumn{4}{|c|}{ Empirical Standard Error Estimates } & \\
\hline Parameter & Estimate & $\begin{array}{l}\text { Standard } \\
\text { Error }\end{array}$ & $\begin{array}{l}\text { P-Value } \\
(\operatorname{Pr}>|\mathrm{Z}|)\end{array}$ & \\
\hline Intercept & 0.8895 & 0.7218 & 0.2178 & \\
\hline PropSamoans & -3.5994 & 0.8669 & $<.0001$ & 0.0273 \\
\hline Certificates & 0.0342 & 0.0051 & $<.0001$ & 1.0348 \\
\hline Degrees & -0.0291 & 0.0086 & 0.0007 & 0.9713 \\
\hline
\end{tabular}

\begin{tabular}{|l|r|r|r|}
\hline \multicolumn{4}{|c|}{ Score Statistics For Type 3 GEE Analysis } \\
\hline \multicolumn{1}{|c|}{ Source } & DF & $\begin{array}{c}\text { Chi- } \\
\text { Square }\end{array}$ & $\begin{array}{c}\text { P-Value } \\
\text { (Pr > ChiSq) }\end{array}$ \\
\hline PropSamoans & 1 & 4.55 & 0.0329 \\
Certificates & 1 & 2.85 & 0.0915 \\
Degrees & 1 & 5.9 & 0.0151 \\
\hline
\end{tabular}

\subsection{The Secondary School Building Effect (2007)}

There are 31 continuous explanatory variables that were used to measure the impact of school building on dropouts. These variables include the school size (TotEnrol), Classrooms, ClassroomArea, Offices, OfficeArea, Storerooms, StoreroomArea, HomeEc, HomeEcArea, Artrooms, ArtroomArea, ScienceLab, ScienceLabArea, Staffrooms, StaffroomArea, Libraries, LibraryArea, Fales (Samoan traditional houses), FaleArea, Halls, HallArea, Workshops, WorkshopArea, Canteens, CanteenArea, ComputerLabs, ComputerLabArea, Toilets, Urinals, Washbasins and Drinkfountains. Despite having all these variables in the full model (see Appendix B), only 4 of them (after many steps of backward elimination for model selection) are significantly associated with the dropout based on the Chi-Square statistics shown in Table 5.30.

\section{Model Interpretation}

Students are less likely to drop out of schools that have more school offices and/or have bigger computer labs (in terms of its area). Strictly speaking, students are 1.3 
$(\mathrm{OR}=1.2726$, which is the inverse of 0.7858$)$ times more likely and $1.005(\mathrm{OR}=$ 1.0052, which is the inverse of 0.9948) times more likely to drop out of schools that have fewer school offices and/or have smaller computer labs respectively. On the other hand, students are $1.004(\mathrm{OR}=1.0040)$ times more likely and $1.14(\mathrm{OR}=$ 1.1442) times more likely to drop out of schools that have larger Science labs (in terms of its area) and/or have more drinking fountains respectively. There maybe some other factors which are not accounted for and reflected in the census survey that are associated with the drinking fountains, for it to be significantly associated with the dropout. The Chi-Square statistics for the full model appears in Table 5.31.

Table 5.30: The Simpler Model for 2007 Secondary School Building Variables

\begin{tabular}{|l|r|r|r|r|}
\hline \multicolumn{4}{|c|}{ Analysis Of GEE Parameter Estimates } & \\
\cline { 1 - 3 } Empirical Standard Error Estimates \\
\cline { 1 - 3 } Parameter & Estimate & $\begin{array}{c}\text { Standard } \\
\text { Error }\end{array}$ & $\begin{array}{c}\text { P-Value } \\
(\mathbf{P r}>|\mathbf{Z}|)\end{array}$ & $\begin{array}{c}\text { Odds } \\
\text { Ratio }\end{array}$ \\
\hline Intercept & -2.0288 & 0.1229 & $<.0001$ & \\
Offices & -0.2411 & 0.0847 & 0.0044 & 0.7858 \\
ScienceLabArea & 0.004 & 0.0018 & 0.0238 & 1.0040 \\
ComputerLabArea & -0.0052 & 0.0023 & 0.0215 & 0.9948 \\
Drinkfountains & 0.1347 & 0.0591 & 0.0226 & 1.1442 \\
\hline
\end{tabular}

\begin{tabular}{|l|r|r|r|}
\hline \multicolumn{4}{|c|}{ Score Statistics For Type 3 GEE Analysis } \\
\hline \multicolumn{1}{|c|}{ Source } & DF & $\begin{array}{c}\text { Chi- } \\
\text { Square }\end{array}$ & $\begin{array}{c}\text { P-Value } \\
(\mathbf{P r}>\mathbf{C h i S q})\end{array}$ \\
\hline Offices & 1 & 4.68 & 0.0305 \\
ScienceLabArea & 1 & 4.23 & 0.0397 \\
ComputerLabArea & 1 & 4.33 & 0.0374 \\
Drinkfountains & 1 & 6.76 & 0.0093 \\
\hline
\end{tabular}


Table 5.31: The Chi-Square Statistics of Full Model for 2007 Secondary School Building Variables

\begin{tabular}{|l|r|r|r|}
\hline \multicolumn{4}{|c|}{ Score Statistics For Type 3 GEE Analysis } \\
\hline \multicolumn{1}{|c|}{ Source } & DF & $\begin{array}{c}\text { Chi- } \\
\text { Square }\end{array}$ & $\begin{array}{c}\text { P-Value } \\
\text { (Pr > ChiSq) }\end{array}$ \\
\hline TotEnrol & 1 & 3.85 & 0.0498 \\
Classrooms & 1 & 2.68 & 0.1014 \\
ClassroomArea & 1 & 1.29 & 0.2559 \\
Offices & 1 & 1.78 & 0.1823 \\
OfficeArea & 1 & 5.61 & 0.0179 \\
Storerooms & 1 & 0.64 & 0.4252 \\
StoreroomArea & 1 & 4.89 & 0.0269 \\
HomeEc & 1 & 2.21 & 0.1368 \\
HomeEcArea & 1 & 0.23 & 0.632 \\
Artrooms & 1 & 0.71 & 0.399 \\
ArtroomArea & 1 & 4.13 & 0.0422 \\
ScienceLab & 1 & 2.97 & 0.0847 \\
ScienceLabArea & 1 & 13.13 & 0.0003 \\
Staffrooms & 1 & 2.5 & 0.1142 \\
StaffroomArea & 1 & 4.08 & 0.0433 \\
Libraries & 1 & 1.4 & 0.2367 \\
LibraryArea & 1 & 0.55 & 0.4571 \\
Fales & 1 & 1.97 & 0.1607 \\
FaleArea & 1 & 3.72 & 0.0536 \\
Halls & 1 & 0.22 & 0.6357 \\
HallArea & 1 & 0.12 & 0.7306 \\
Workshops & 1 & 0.14 & 0.7061 \\
WorkShopArea & 1 & 0.16 & 0.6868 \\
Canteens & 1 & 0.01 & 0.9415 \\
CanteenArea & 1 & 1.27 & 0.2605 \\
ComputerLabs & 1 & 0.25 & 0.6178 \\
ComputerLabArea & 1 & 7.84 & 0.0051 \\
Toilets & 1 & 1.73 & 0.1889 \\
Urinals & 1 & 0.15 & 0.6985 \\
Washbasins & 1 & 1.75 & 0.1861 \\
Drinkfountains & 1 & 8.17 & 0.0043 \\
\hline
\end{tabular}


The study wishes to investigate whether school facilities are associated with dropping out of school. Hence, 26 continuous explanatory variables were used to measure the effect of the school facility on dropout. These variables include the school size (TotEnrol), Desks, Seats, TeacherDesks, TeacherChairs, Blackboards, StaffTables, StaffChairs, StaffCupboards, StaffBlackboards, StaffPinboards, StaffSinks, Buses, trucks, Vehicles, Duplicators, Photocopiers, Computers, Radios, TVs, Projectors, FireExtinguisher, FirstAid, Phones, Fax and Internet. During the model selection process (which uses the backward elimination method to remove one insignificant variable one at a time), the study found only 2 variables that are significantly associated with the dropout based on the Chi-Square statistics shown in Table 5.32. They are the number of trucks and radios a school has.

\section{Model Interpretation}

Students are less likely to drop out of schools that have more trucks for transportation, and more radios for class and school activities. That is, students are 1.72 (OR = 1.7206, which is the inverse of 0.5812) times more likely and 1.08 ( $\mathrm{OR}=1.0757$, which is the inverse of 0.9296) times more likely to quit schools that have fewer trucks for transportation and/or have fewer radios for class and school activities respectively. The Chi-Square statistics of the full model (see Appendix B) is presented in Table 5.33.

Table 5.32: The Simpler Model for 2007 Secondary School Facility Variables

\begin{tabular}{|lrrrr|}
\hline \multicolumn{4}{|c|}{ Analysis Of GEE Parameter Estimates } & \multirow{2}{*}{} \\
\cline { 1 - 3 } Empirical Standard Error Estimates & \multirow{2}{*}{ Odds } \\
Parameter & Estimate & $\begin{array}{c}\text { Standard } \\
\text { Error }\end{array}$ & $\begin{array}{c}\text { P-Value } \\
(\text { Pr }>|\mathbf{Z}|)\end{array}$ & Ratio \\
\hline Intercept & -1.7658 & 0.1031 & $<.0001$ & \\
Trucks & -0.5426 & 0.1637 & 0.0009 & 0.5812 \\
Radios & -0.073 & 0.031 & 0.0185 & 0.9296 \\
\hline
\end{tabular}

\begin{tabular}{|l|c|c|c|}
\hline \multicolumn{4}{|c|}{ Score Statistics For Type 3 GEE Analysis } \\
\hline Source & DF & $\begin{array}{c}\text { Chi- } \\
\text { Square }\end{array}$ & $\begin{array}{c}\text { P-Value } \\
(\mathbf{P r}>\text { ChiSq })\end{array}$ \\
\hline Trucks & 1 & 4.05 & 0.0442 \\
Radios & 1 & 4.44 & 0.0351 \\
\hline
\end{tabular}


Table 5.33: The Chi-Square Statistics of Full Model for 2007 Secondary School Facility Variables

\begin{tabular}{|l|r|r|r|}
\hline \multicolumn{4}{|c|}{ Score Statistics For Type 3 GEE Analysis } \\
\hline \multicolumn{1}{|c|}{ Source } & DF & $\begin{array}{c}\text { Chi- } \\
\text { Square }\end{array}$ & $\begin{array}{c}\text { P-Value } \\
\text { (Pr > ChiSq) }\end{array}$ \\
\hline TotEnrol & 1 & 1.55 & 0.2135 \\
Desks & 1 & 1.6 & 0.206 \\
Seats & 1 & 3.5 & 0.0612 \\
TeacherDesks & 1 & 0.07 & 0.7896 \\
TeacherChairs & 1 & 0.11 & 0.741 \\
Blackboards & 1 & 0.25 & 0.6168 \\
StaffTables & 1 & 2.88 & 0.0896 \\
StaffChairs & 1 & 5.79 & 0.0162 \\
StaffCupboards & 1 & 3.37 & 0.0663 \\
StaffBlackboards & 1 & 0.92 & 0.3376 \\
StaffPinboards & 1 & 1.8 & 0.1795 \\
StaffSinks & 1 & 2.7 & 0.1004 \\
Buses & 1 & 2.03 & 0.1545 \\
Trucks & 1 & 6.61 & 0.0102 \\
Vehicles & 1 & 1 & 0.3183 \\
Duplicators & 1 & 0.42 & 0.5179 \\
Photocopiers & 1 & 0.07 & 0.79 \\
Computers & 1 & 3.39 & 0.0654 \\
Radios & 1 & 5.05 & 0.0246 \\
TVs & 1 & 2.71 & 0.0995 \\
Projectors & 1 & 0.06 & 0.8095 \\
FireExtinguisher & 1 & 3.97 & 0.0463 \\
FirstAid & 1 & 2.03 & 0.1545 \\
Phones & 1 & 0.01 & 0.9077 \\
Fax & 1 & 5.98 & 0.0145 \\
Internet & 1 & 0.52 & 0.4692 \\
\hline
\end{tabular}




\section{Chapter 6}

\section{Discussions and Conclusion}

This chapter discusses the results and findings of this study as well as issues and difficulties that the study had faced. Some useful recommendations for the betterment and reduction of the dropout crisis in Samoan schools are also presented here.

\subsection{Results}

The results and findings of this study were based on the data collected annually by the Samoan Ministry of Education, Sports and Culture through census forms, which means actual facts and reasons why Samoan students were dropping out of schools are not disclosed and can not be analysed, unless a follow up interview on those who dropped out has taken place.

\section{The Year Level and Region Effects}

The study found that the dropout rates varied across all the school year levels. However, higher dropout rates were found at Year 13 level across all the years except for the years 1998 until 2002. For these five consecutive years the odds of dropping out of Year 12 level were much higher than that of any other year level. Nonetheless, the Year 2 level as well had the higher dropout rates when compared to Year 8 and other school year levels in between across all the years.

The higher number of dropouts at Year 13 could be resulted from many students who failed or did not achieve required grades (or marks) from the Year 12 National School Certificate Exam to enter the Year 13 level. Likewise, higher number of dropouts for Year 12 could also be an outcome of failing the Year 11 National Exam. This is pretty 
much consistent with the findings presented in section 2.2 of the literature review chapter. As reported in section 2.2, failing exit exams cause more students to give up school early. These students may have decided to either look for a paying job to help out with families, migrate overseas, enrol in or transfer to other schools or repeat the Year 12 level again. Repeat the same school year level also increases the dropout rate as reported by section 2.2, which may also be another reason of having higher dropout rates for Year 12. Some of the dropout students may have decided to take apprenticeship courses so they could get qualifications and skills, and seek job opportunities to utilise and apply these skills. Moreover, some of them may have opted to stay home and look after family businesses.

In the case of more students dropping out at Year 2 probably because this is the children's first year in primary school and he/she needs to adjust to the new environment. This is consistent with findings from the literature review chapter as seen in section 2.4; where higher dropout rates have been discovered in early primary grades or school year levels. Some of these new or first year students may not like the school environment for some reasons (like the ones mentioned in section 2.2) such as too many students; teachers or peers are not very friendly and disliked school subjects and decided to transfer to another school.

The study also found that more students were also dropping out of Year 11 and Year 10. This could be resulted from either students being suspended or expelled from schools for whatever reason, or they had been transferred or migrated to other schools. This reflects that the dropout rates were much higher in secondary schools than in primary schools. It is possible to say that this is because at secondary level majority of the students were at least 14 years old which is outside the limit of the age group (5-14) for compulsory education. As reported by section 2.8 raising the upper limit of the compulsory education age group might be helpful in reducing the higher dropout rates at secondary schools.

For the region effects the study found significant associations between the dropout and regions in the following calendar years; 1995, 1996, 1997, 1998, 1999, 2001, 2003 and 2006. For these years the dropout rates were much higher in the Rest of Upolu region than in Savaii and the Apia urban area, even though dropout rates were higher in Savaii than Apia urban region. Strictly speaking, students were less likely to depart school prematurely in the Apia urban area than in both Rest of Upolu and Savaii areas. For years that showed no significant relationships with the outcome variable the same trend was also seen. Thus more students left schools prematurely in rural areas than in the urban or the town area. These results are consistent with some of the findings as indicated in section 2.3, whereby higher dropout rates were discovered in rural areas than urban areas. Reasons that might relate to higher dropout rates in Samoa rural areas could be similar to those reported in section 2.2. In Samoa rural areas many families have financial difficulties or are very low in terms of socioeconomic status, and have at least four family members. These could be some of the 
reasons on top of other reasons stipulated in section 2.2 associated with higher dropout rates in Samoa rural areas.

The study also believes that most of these dropouts from both the Rest of Upolu and Savaii regions had transferred to schools in the Apia urban area as a result of family mobility or being migrated to the town area for better living. Another possibility could be Apia urban schools were well equipped in terms of school buildings and facilities as well as resources in contrast to rural areas.

\section{The School Level Effect}

The study found no significant relationship between the response variable and the school level across all years, given the year levels. However, when comparisons were made between the three levels (primary, primary-secondary and secondary) more students were dropping out of secondary schools than both the primary and primarysecondary. This result is nonconflicting with findings presented in section 2.4 , where higher dropout rates were found in high schools in USA and Botswana due to factors mentioned in section 2.2. One of the school factors for sure that pushed students out of Samoan secondary schools is the national exit exams such as (Year 8 and Year 11 National Exams, as well as Year 12 School Certificate Exams), as Samoa has a competitive test or exam based educational system. The quality of teachers in terms of qualification and attitudes (as there were not that many teachers with degrees teaching at rural secondary schools) could be another possibility of having higher dropout rates in secondary schools than primary and primary/secondary schools. It is important to note that there are more secondary schools in rural areas (comprised of Rest of Upolu and Savaii regions) than in urban area (Apia urban). Other factors such as financial constraints and distance of students' homes from schools, as reported by section 2.2, could also be other reasons why Samoan students were more likely to quit secondary schools earlier than expected.

\section{The School Status Effect}

There were significant associationts found between the response variable and the school status in the following calendar years: 1999, 2000 and 2003. For these years, the dropout rates were higher for Government schools than both the Mission and Private schools. The same years while controlling the effect of the Government 
schools, Mission schools students were more likely to drop out than Private schools students.

There was no significant relationship between the dropouts and school status for other calendar years. However, when comparisons were made between the three school status the Government school students were more likely to drop out than both the Mission and Private school students. In other words, Private school students were less likely to withdraw from schools compare to those from both the Government and Mission. Even though it is true but the differences are insignificant. These findings are conformable to what the literature says in section 2.5, whereby higher dropout rates for Public schools in the United States and Australia as compared to Catholic and Private schools.

The study believes that one of the reasons for Government schools having the highest number of dropouts is because of National Exit exams as mentioned in section 2.2. It is important to note that there are more Government schools in rural areas (as in Rest of Upolu and Savaii regions) than in the urban area (Apia urban). Low socioeconomic backgrounds of students and possibly distance of students' homes from schools could be some other reasons associated with higher dropout rates for Government schools than Mission and Private schools in particular. Lower dropout rates were discovered in Private schools as most of the students were from well to do families and high socio-economic backgrounds. Furthermore, most of Private school teachers are highly qualified and are being paid well more than what Government and Mission school teachers are getting. Teacher salary as reported in section 2.6 is one of the factors associated with the dropouts. These could be other reasons as why Private schools have lower dropout rates than Government and Mission schools.

\section{The Student-Teacher Ratio Effect}

Interestingly, the study discovered that across all the calendar years, the studentteacher ratio was significantly associated with the dropouts. Surprisingly, students were less likely to drop out of schools with higher student-teacher ratios. Higher student-teacher ratio means larger school in terms of enrolment and fewer teachers. This is noncompatible with some of the findings in section 2.6 of the literature review chapter, whereby schools with higher student-teacher ratios have higher dropout rates as well. However, other studies by Branham (2004), Ilon \& Moock (1991) and Provasnik et al (2007) support our findings. Provasnik's et al (2007) findings are similar to ours as they indicate that more students were dropping out of public schools with lower student-teacher ratio, as larger percentages of public school students attended very small schools in rural America. For Samoan schools lower dropout rates were discovered in the Apia urban area as it has larger schools as compare to the Rest 
of Upolu and Savaii regions. Moreover, Apia urban schools have many extracurricular activities in which students are engaged in than schools in the rural areas, Rest of Upolu and Savaii regions.

Another reason for having lower dropout rates in the Apia urban schools could be the teacher qualification and teaching experience (Lee \& Burkam, 2003 Ilon \& Moock, 1991), as there are more Degree holders teaching in Apia urban schools than in the Rest of Upolu and Savaii regions. There are other reasons such as, socio-economic status of students, and some school factors that could associate with having lower dropout rates for schools that have higher student-teacher ratios.

\section{The School Size (Total Enrolment) Effect}

As mentioned earlier that higher student-teacher ratios are caused by larger enrolments and fewer teachers. From the results students were less likely to drop out of larger schools than in small schools. It is contrary to some of the findings in section 2.6, however other findings such as those mentioned in the "Student-Teacher Ratio Effect" subsection above, underpins our claim. Factors that could associate with lower dropout rates in larger schools in Samoa include students' participation in extracurricular activities, teacher qualification and experience, student-teacher relationship, parents and family support and other school factors.

\section{The Secondary School Teacher Variables Effect (2007)}

From the results students were less likely to leave school prematurely when there are more Samoan teachers and more teachers with Degrees in the school. It could be argued that larger schools in the Apia urban region are benefited from teachers with Degrees or higher qualifications as compared to their counterparts in the Rest of Upolu and Savaii regions. Many of these teachers have got years of experience and are high quality too. These school teachers with higher qualifications are earning more than their peers with just the Teaching Certificate unless they are in a position of responsibilities. These could be some of the reasons, which are consistent with the findings in section 2.2 and 2.6, associated with lower dropout rates for Samoan schools having more Samoan and Degree holder teachers in it.

The gender of the teachers as well as teachers with teaching certificates were not significantly associated with the dropouts. 
The result shows that students were less likely to drop out of schools with more offices and have bigger computer labs (in terms of its area). On the other hand students were more likely to give up schools that have bigger Science laboratories (in terms of its area) and more drinking fountains. With the extensive usage of computers in schools and many students attend and like computing courses, the study assumes that because of this, schools then built bigger computer labs to cater for these students. In saying that greater school buildings or resources would keep students in schools until completion. This is confirmed by other studies as mentioned in section 2.7. However, for having more students dropping out of schools with bigger Science laboratories and more drinking fountains is a concern. We could only assume that may be schools with low student-teacher ratios and have bigger Science laboratories are associated with higher dropout rates. But for higher dropout rates for schools with more number of drinking fountains, the study assumes that there may be some other factors not accounted for that are associated with the number of drinking fountains, which in turn triggers the dropout rate in the positive direction.

All the other school building variables as mentioned in section 5.7 were not significantly related to the dropouts.

\section{The Secondary School Facility Effect (2007)}

From the results students were less likely to drop out of schools that have more trucks and radios. Some of the schools in Samoa especially those in the Apia urban area have trucks, which are often used to transport students to and from sports venues and other school activities. This could be one of the reasons why students were less likely to quit schools that have more trucks. Nevertheless, Samoan schools have school programs that broadcast live on radios and have many school or class activities that need radio or stereos for it. These could be reasons why dropout rates were low for schools that have more radios.

All the other school facility variables as mentioned in section 5.8 were not significantly associated with the dropouts. 
The study was based on the data collected by the MESC through its PPRD division. A standard questionnaire called the Census form was used to collect the required information needed by the Ministry for statistical, administrative and educational purposes. The study also wished to explore and investigate other aspects or characteristics of the dropout crisis in Samoa such as the proportions of dropouts by gender (male and female), by age and by ethnicity. The study also had an interest in finding out reasons why Samoan students particularly at primary and secondary schools dropping out of school. However, because of the way the questions in the census form were set out these aims were not met. That is, information about the social economic backgrounds of students was not available at the time because of limited data provided through administrative records in terms of census forms.

In order to achieve this, further researches must be taken in the future to survey and identify these areas such as which of the two sexes (male or female) has got the higher proportion of dropouts. Moreover, an investigation about the dropout by age and ethnicity must also be carried out in Samoa and then compare them to the outcome of surveys being carried out in other countries.

\subsection{Recommendations}

The findings and results of this study were based on the census data collected through census forms by the MESC annually; and because of this, records of transferred and migrated students are not accounted for as reasons why negative dropout rates still appear in the MESC Statistical Digest every year. Therefore the study wishes to recommend that the census form should be reviewed in order to include such missing information, which helps improve the dropout rate calculation for Samoan schools. One way to improve this is to assign a unique student identification number as suggested by Tyler \& Lofstrom (2009) to every student when they first enrol or enter into the school system. That way, the MESC will be able to keep track of them and more importantly these student identification numbers (or student education number) will also produce accurate enrolment, completion and dropout statistics.

Because of higher dropout rates at Secondary school level, the study wishes to suggest the increasing of the upper limit of the compulsory age group for Samoan students. Further, we would also like to propose proper and thorough investigation of why rural schools have higher dropout rates especially for Government schools. Furthermore, upgrading the quality of school teachers in terms of qualification, attitudes and skills 
and couple it with increasing the teachers' salary might be helpful in reducing the dropout rates. Nevertheless, a fair distribution of highly qualified teachers to all the schools in both rural and urban areas should also be considered. Upgrading some school building and facility as well as some other school resources might also work in reducing the dropout rate.

Other dropout prevention and intervention programmes such as, eliminating school exit exams and others that are mentioned in section 2.8 , could also be useful in reducing the dropout rates for Samoan schools. Last but not the least, the study wishes to advise the MESC to review "the dropout definition" given in the Statistical Digest.

\subsection{Conclusion}

The work carried out in this study is very useful in portraying the dropout phenomena in Samoan schools, particularly at primary and secondary level. From this work we managed to identify areas and factors, as indicated in the main purposes of the study in chapter one, that are associated with the dropouts. In addition, from the results of this study we also managed to answer our research questions and/or hypothesis stated earlier in chapter one.

We conclude that students were more likely to drop out of rural schools than urban schools. Sadly, the majority of these students were from Secondary schools, which shows that the dropout rate grows with age and grade or school year level. That is the older or the higher the school year level (grade) the student is the more likely he or she is to depart school prematurely. More depressingly, students attending Government schools were more affected as compared to their peers in the Mission and Private schools in particular. Despite very low school fees paid by Government schools' students and free school stationery received by these students, and yet still very high dropout rates were discovered for Government schools. This could perhaps link to other factors like the school size and student-teacher ratio. It has been discovered that students were less likely to quit larger schools and those with higher student-teacher ratio. Maybe some other school demographic factors like the gender and ethnicity of the teacher, as well as the qualification of the teachers are triggering these low dropout rates for larger schools and those with higher student-teacher ratio. In our discovery, having more Samoan and more degree holder teachers in a Samoan classroom will significantly decline the dropout rate. Unfortunately, the gender of the teachers had nothing to do with the dropout.

We have also managed to identify some school building and facility variables that were significantly associated with the dropout. Fewer students were dropping out of schools with more school offices and had larger Computer Lab (in terms of its area). 
Moreover, schools with more trucks for transportation and more radios for school programs and activities were less likely to get affected with the dropout problem. However, schools with larger Science Laboratories (in terms of its area) and more drinking fountains were more likely to get affected with the dropout problem. With the latter the study assumes that there may be some other factors associated with the drinking fountains that were not picked up during the data collection, as due to the way the census form questions were posed.

The dropout problem in Samoa is solvable if necessary actions and/or sequences of effective prevention and intervention programmes are taken before it becomes a catastrophic situation. Otherwise, "only lower economic wages and irregular employment are the promises of the future that face the dropouts of yesterday, today and tomorrow" (Campbell, 1966). 


\section{APPENDICES}

\section{A. All Schools' Other Models (1995 - 2006)}

A. R.1: Estimates and Odds Ratio for Year Level and Region in 1996

\begin{tabular}{|c|c|c|c|c|c|}
\hline \multicolumn{5}{|c|}{ Analysis Of GEE Parameter Estimates } & \multirow[b]{3}{*}{$\begin{array}{l}\text { Odds } \\
\text { Ratio }\end{array}$} \\
\hline \multicolumn{5}{|c|}{ Empirical Standard Error Estimates } & \\
\hline Parameter & Level & Estimate & $\begin{array}{l}\text { Standard } \\
\text { Error }\end{array}$ & $\begin{array}{l}\text { P-Value } \\
(\operatorname{Pr}>|Z|)\end{array}$ & \\
\hline Intercept & & -3.4091 & 0.1828 & $<.0001$ & \\
\hline YearLevel & Yr10 & 1.3193 & 0.2404 & $<.0001$ & 3.7408 \\
\hline YearLevel & Yr11 & 0.7089 & 0.3897 & 0.0689 & 2.0318 \\
\hline YearLevel & Yr12 & 1.1172 & 0.4692 & 0.0173 & 3.0563 \\
\hline YearLevel & Yr13 & 2.3425 & 0.6753 & 0.0005 & 10.4072 \\
\hline YearLevel & Yr2 & 1.1384 & 0.2132 & $<.0001$ & 3.1218 \\
\hline YearLevel & Yr3 & 0.4134 & 0.1796 & 0.0214 & 1.5119 \\
\hline YearLevel & Yr4 & 0.2173 & 0.2167 & 0.3159 & 1.2427 \\
\hline YearLevel & Yr5 & 0.4641 & 0.2105 & 0.0275 & 1.5906 \\
\hline YearLevel & Yr6 & 0.7357 & 0.2264 & 0.0012 & 2.0869 \\
\hline YearLevel & Yr7 & 0.5009 & 0.1957 & 0.0105 & 1.6502 \\
\hline YearLevel & Yr8 & 0 & 0 & & 1.0000 \\
\hline Region & Apia Urban & -0.501 & 0.2046 & 0.0144 & 0.6059 \\
\hline Region & Rest of Upolu & 0.4601 & 0.139 & 0.0009 & 1.5842 \\
\hline Region & Savaii & 0 & 0 & 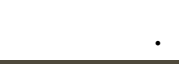 & 1.0000 \\
\hline
\end{tabular}

\begin{tabular}{|l|r|r|r|}
\hline \multicolumn{4}{|c|}{ Score Statistics For Type 3 GEE Analysis } \\
\hline Source & DF & $\begin{array}{c}\text { Chi- } \\
\text { Square }\end{array}$ & $\begin{array}{c}\text { P-Value } \\
\text { (Pr > ChiSq) }\end{array}$ \\
\hline YearLevel & \multicolumn{1}{|c|}{5} & 50.87 & $<.0001$ \\
Region & 2 & 24.11 & $<.0001$ \\
\hline
\end{tabular}


A. R.2: Estimates and Odds Ratio for Year Level and Region in 1997

\begin{tabular}{|l|l|r|r|r|r|}
\hline \multicolumn{7}{|c|}{ Analysis Of GEE Parameter Estimates } & \multirow{2}{*}{} \\
\cline { 1 - 5 } Parameter & Level & Estimate & $\begin{array}{c}\text { Standard } \\
\text { Error }\end{array}$ & $\begin{array}{c}\text { P-Value } \\
\text { (Pr }>|Z|)\end{array}$ & \multicolumn{1}{|c}{$\begin{array}{l}\text { Odds } \\
\text { Ratio }\end{array}$} \\
\hline Intercept & & -3.5437 & 0.2347 & $<.0001$ & \\
YearLevel & Yr10 & 1.4494 & 0.2749 & $<.0001$ & 4.2606 \\
YearLevel & Yr11 & 0.9735 & 0.3815 & 0.0107 & 2.6472 \\
YearLevel & Yr12 & 1.5412 & 0.4074 & 0.0002 & 4.6702 \\
YearLevel & Yr13 & 3.5273 & 0.3421 & $<.0001$ & 34.0320 \\
YearLevel & Yr2 & 1.4021 & 0.2392 & $<.0001$ & 4.0637 \\
YearLevel & Yr3 & 0.5229 & 0.239 & 0.0287 & 1.6869 \\
YearLevel & Yr4 & 0.6196 & 0.2276 & 0.0065 & 1.8582 \\
YearLevel & Yr5 & 0.6568 & 0.2493 & 0.0084 & 1.9286 \\
YearLevel & Yr6 & 0.5345 & 0.2366 & 0.0239 & 1.7066 \\
YearLevel & Yr7 & 0.8511 & 0.2324 & 0.0002 & 2.3422 \\
YearLevel & Yr8 & 0 & 0 &. & 1.0000 \\
Region & Apia Urban & -0.741 & 0.2316 & 0.0014 & 0.4766 \\
Region & Rest of Upolu & 0.1455 & 0.143 & 0.3088 & 1.1566 \\
Region & Savaii & 0 & 0 &. & 1.0000 \\
\hline
\end{tabular}

\begin{tabular}{|l|r|r|r|}
\hline \multicolumn{4}{|c|}{ Score Statistics For Type 3 GEE Analysis } \\
\hline Source & DF & $\begin{array}{c}\text { Chi- } \\
\text { Square }\end{array}$ & $\begin{array}{c}\text { P-Value } \\
\text { (Pr }>\text { ChiSq) }\end{array}$ \\
\hline YearLevel & 10 & 52.06 & $<.0001$ \\
Region & 2 & 17.52 & 0.0002 \\
\hline
\end{tabular}


A. R.3: Estimates and Odds Ratio for Year Level and Region in 1998

\begin{tabular}{|l|l|r|r|r|r|}
\hline \multicolumn{5}{|c|}{ Analysis Of GEE Parameter Estimates } & \multirow{2}{*}{} \\
\cline { 1 - 4 } Parameter & Level & Estimate & $\begin{array}{c}\text { Standard } \\
\text { Error }\end{array}$ & $\begin{array}{c}\text { P-Value } \\
(\text { Pr }>|Z|)\end{array}$ & $\begin{array}{l}\text { Odds } \\
\text { Ratio }\end{array}$ \\
\hline Intercept & & -3.6028 & 0.2474 & $<.0001$ & \\
YearLevel & Yr10 & 1.3298 & 0.284 & $<.0001$ & 3.7803 \\
YearLevel & Yr11 & 0.5557 & 0.4594 & 0.2264 & 1.7432 \\
YearLevel & Yr12 & 2.0938 & 0.4165 & $<.0001$ & 8.1157 \\
YearLevel & Yr13 & 1.7852 & 0.5507 & 0.0012 & 5.9608 \\
YearLevel & Yr2 & 1.4147 & 0.2419 & $<.0001$ & 4.1153 \\
YearLevel & Yr3 & 0.6548 & 0.2533 & 0.0097 & 1.9248 \\
YearLevel & Yr4 & 0.5033 & 0.225 & 0.0253 & 1.6542 \\
YearLevel & Yr5 & 0.8844 & 0.2427 & 0.0003 & 2.4215 \\
YearLevel & Yr6 & 0.6005 & 0.2454 & 0.0144 & 1.8230 \\
YearLevel & Yr7 & 0.7287 & 0.2358 & 0.002 & 2.0724 \\
YearLevel & Yr8 & 0 & 0 &. & 1.0000 \\
Region & Apia Urban & -0.6455 & 0.2741 & 0.0185 & 0.5244 \\
Region & Rest of Upolu & 0.0166 & 0.1625 & 0.9187 & 1.0167 \\
Region & Savaii & 0 & 0 &. & 1.0000 \\
\hline
\end{tabular}

Score Statistics For Type 3 GEE Analysis

\begin{tabular}{|l|r|r|r|}
\hline \multicolumn{1}{|c|}{ Source } & DF & $\begin{array}{c}\text { Chi- } \\
\text { Square }\end{array}$ & $\begin{array}{c}\text { P-Value } \\
\text { (Pr }>\text { ChiSq) }\end{array}$ \\
\hline YearLevel & 10 & 42.83 & $<.0001$ \\
Region & 2 & 7.91 & 0.0192 \\
\hline
\end{tabular}


A. R.4: Estimates and Odds Ratio for Year Level and Region in 1999

\begin{tabular}{|c|c|c|c|c|c|}
\hline \multicolumn{5}{|c|}{ Analysis Of GEE Parameter Estimates } & \multirow[b]{3}{*}{$\begin{array}{l}\text { Odds } \\
\text { Ratio }\end{array}$} \\
\hline \multicolumn{5}{|c|}{ Empirical Standard Error Estimates } & \\
\hline Parameter & Level & Estimate & $\begin{array}{l}\text { Standard } \\
\text { Error }\end{array}$ & $\begin{array}{c}\text { P-Value } \\
(\mathrm{Pr}>|\mathrm{Z}|)\end{array}$ & \\
\hline Intercept & & -3.6403 & 0.2562 & $<.0001$ & \\
\hline YearLevel & Yr10 & 1.4164 & 0.3248 & $<.0001$ & 4.1223 \\
\hline YearLevel & Yr11 & 0.9504 & 0.428 & 0.0264 & 2.5867 \\
\hline YearLevel & Yr12 & 2.0875 & 0.401 & $<.0001$ & 8.0647 \\
\hline YearLevel & Yr13 & 1.5373 & 0.5307 & 0.0038 & 4.6520 \\
\hline YearLevel & Yr2 & 1.3198 & 0.2287 & $<.0001$ & 3.7427 \\
\hline YearLevel & Yr3 & 0.6582 & 0.2457 & 0.0074 & 1.9313 \\
\hline YearLevel & Yr4 & 0.7337 & 0.2161 & 0.0007 & 2.0828 \\
\hline YearLevel & Yr5 & 0.7558 & 0.2394 & 0.0016 & 2.1293 \\
\hline YearLevel & Yr6 & 0.6397 & 0.2257 & 0.0046 & 1.8959 \\
\hline YearLevel & Yr7 & 1.0013 & 0.2215 & $<.0001$ & 2.7218 \\
\hline YearLevel & Yr8 & 0 & 0 & & 1.0000 \\
\hline Region & Apia Urban & -0.7367 & 0.3053 & 0.0158 & 0.4787 \\
\hline Region & Rest of Upolu & -0.0289 & 0.2058 & 0.8882 & 0.9715 \\
\hline Region & Savaii & 0 & 0 & & 1.0000 \\
\hline
\end{tabular}

Score Statistics For Type 3 GEE Analysis

\begin{tabular}{|l|r|r|r|}
\hline \multicolumn{1}{|c|}{ Source } & DF & $\begin{array}{c}\text { Chi- } \\
\text { Square }\end{array}$ & $\begin{array}{c}\text { P-Value } \\
\text { (Pr }>\text { ChiSq) }\end{array}$ \\
\hline YearLevel & 10 & 42.06 & $<.0001$ \\
Region & 2 & 7.35 & 0.0254 \\
\hline
\end{tabular}


A. R.5: Estimates and Odds Ratio for Year Level and Region in 2000

\begin{tabular}{|l|l|r|r|r|r|}
\hline \multicolumn{5}{|c|}{ Analysis Of GEE Parameter Estimates } & \multirow{2}{*}{} \\
\cline { 1 - 4 } Parameter & Level & Estimate & $\begin{array}{c}\text { Standard } \\
\text { Error }\end{array}$ & $\begin{array}{r}\text { P-Value } \\
(\text { Pr }>|\mathbf{Z}|)\end{array}$ & $\begin{array}{l}\text { Odds } \\
\text { Ratio }\end{array}$ \\
\hline Intercept & & -3.4176 & 0.2285 & $<.0001$ & \\
YearLevel & Yr10 & 1.2131 & 0.2891 & $<.0001$ & 3.3639 \\
YearLevel & Yr11 & 0.673 & 0.3688 & 0.068 & 1.9601 \\
YearLevel & Yr12 & 1.598 & 0.3819 & $<.0001$ & 4.9431 \\
YearLevel & Yr13 & 0.6363 & 0.5662 & 0.2611 & 1.8895 \\
YearLevel & Yr2 & 1.1604 & 0.2277 & $<.0001$ & 3.1912 \\
YearLevel & Yr3 & 0.3616 & 0.2294 & 0.115 & 1.4356 \\
YearLevel & Yr4 & 0.4502 & 0.2175 & 0.0385 & 1.5686 \\
YearLevel & Yr5 & 0.645 & 0.2601 & 0.0131 & 1.9060 \\
YearLevel & Yr6 & 0.6295 & 0.2195 & 0.0041 & 1.8767 \\
YearLevel & Yr7 & 0.6421 & 0.2275 & 0.0048 & 1.9005 \\
YearLevel & Yr8 & 0 & 0 &. & 1.0000 \\
Region & Apia Urban & -0.1055 & 0.2067 & 0.6096 & 0.8999 \\
Region & Rest of Upolu & 0.1875 & 0.1583 & 0.2362 & 1.2062 \\
Region & Savaii & 0 & 0 &. & 1.0000 \\
\hline
\end{tabular}

\begin{tabular}{|l|r|rr|}
\hline \multicolumn{4}{|c|}{ Score Statistics For Type 3 GEE Analysis } \\
\hline Source & DF & $\begin{array}{r}\text { Chi- } \\
\text { Square }\end{array}$ & $\begin{array}{r}\text { P-Value } \\
(\mathbf{P r}>\text { ChiSq) }\end{array}$ \\
\hline YearLevel & 10 & 43.37 & $<.0001$ \\
Region & 2 & 2.31 & 0.3156 \\
\hline
\end{tabular}


A. R.6: Estimates and Odds Ratio for Year Level and Region in 2001

\begin{tabular}{|l|l|r|r|r|r|}
\hline \multicolumn{7}{|c|}{ Analysis Of GEE Parameter Estimates } & \multirow{2}{*}{} \\
\cline { 1 - 5 } Parameter & Level & Estimate & $\begin{array}{c}\text { Standard } \\
\text { Error }\end{array}$ & $\begin{array}{c}\text { P-Value } \\
(\mathbf{P r}>|\mathbf{Z}| \mathbf{)}\end{array}$ & $\begin{array}{l}\text { Odds } \\
\text { Ratio }\end{array}$ \\
\hline Intercept & & -3.3455 & 0.1824 & $<.0001$ & \\
YearLevel & Yr10 & 1.0119 & 0.246 & $<.0001$ & 2.7508 \\
YearLevel & Yr11 & 0.9968 & 0.2934 & 0.0007 & 2.7096 \\
YearLevel & Yr12 & 1.2348 & 0.3221 & 0.0001 & 3.4377 \\
YearLevel & Yr13 & 1.0395 & 0.4724 & 0.0278 & 2.8278 \\
YearLevel & Yr2 & 0.837 & 0.1994 & $<.0001$ & 2.3094 \\
YearLevel & Yr3 & 0.3455 & 0.2557 & 0.1766 & 1.4127 \\
YearLevel & Yr4 & 0.2271 & 0.186 & 0.2221 & 1.2550 \\
YearLevel & Yr5 & 0.2675 & 0.2044 & 0.1908 & 1.3067 \\
YearLevel & Yr6 & 0.1289 & 0.1974 & 0.5137 & 1.1376 \\
YearLevel & Yr7 & 0.4587 & 0.1822 & 0.0118 & 1.5820 \\
YearLevel & Yr8 & 0 & 0 &. & 1 \\
Region & Apia Urban & -0.2311 & 0.1912 & 0.2268 & 0.7937 \\
Region & Rest of Upolu & 0.1959 & 0.155 & 0.2065 & 1.2164 \\
Region & Savaii & 0 & 0 &. & 1 \\
\hline
\end{tabular}

\begin{tabular}{|l|r|r|r|}
\hline \multicolumn{4}{|c|}{ Score Statistics For Type 3 GEE Analysis } \\
\hline Source & DF & $\begin{array}{c}\text { Chi- } \\
\text { Square }\end{array}$ & $\begin{array}{r}\text { P-Value } \\
\text { (Pr > ChiSq) }\end{array}$ \\
\hline YearLevel & \multicolumn{1}{|c}{30} & 38.31 & $<.0001$ \\
Region & 2 & 6.43 & 0.0401 \\
\hline
\end{tabular}


A. R.7: Estimates and Odds Ratio for Year Level and Region in 2002

\begin{tabular}{|l|lrrrr|r|}
\hline \multicolumn{7}{|c|}{ Analysis Of GEE Parameter Estimates } \\
\cline { 1 - 4 } Parameter & Level & Estimate & $\begin{array}{c}\text { Standard } \\
\text { Error }\end{array}$ & $\begin{array}{c}\text { P-Value } \\
\text { (Pr > |Z|) }\end{array}$ & $\begin{array}{l}\text { Odds } \\
\text { Ratio }\end{array}$ \\
\hline Intercept & & -3.4688 & 0.1727 & $<.0001$ & \\
YearLevel & Yr10 & 0.8032 & 0.2375 & 0.0007 & 2.2327 \\
YearLevel & Yr11 & 1.0613 & 0.3156 & 0.0008 & 2.8901 \\
YearLevel & Yr12 & 0.7906 & 0.4221 & 0.0611 & 2.2047 \\
YearLevel & Yr13 & 0.6855 & 0.4152 & 0.0988 & 1.9848 \\
YearLevel & Yr2 & 0.7762 & 0.1903 & $<.0001$ & 2.1732 \\
YearLevel & Yr3 & 0.0582 & 0.2592 & 0.8225 & 1.0599 \\
YearLevel & Yr4 & -0.1611 & 0.197 & 0.4135 & 0.8512 \\
YearLevel & Yr5 & 0.2985 & 0.2008 & 0.1372 & 1.3478 \\
YearLevel & Yr6 & 0.0192 & 0.1995 & 0.9233 & 1.0194 \\
YearLevel & Yr7 & 0.1952 & 0.1956 & 0.3184 & 1.2156 \\
YearLevel & Yr8 & 0 & 0 &. & 1.0000 \\
Region & Apia Urban & 0.0317 & 0.1993 & 0.8738 & 1.0322 \\
Region & Rest of Upolu & 0.3257 & 0.1618 & 0.0441 & 1.3850 \\
Region & Savaii & 0 & 0 &. & 1.0000 \\
\hline
\end{tabular}

\begin{tabular}{|l|r|r|r|}
\hline \multicolumn{4}{|c|}{ Score Statistics For Type 3 GEE Analysis } \\
\hline \multicolumn{1}{|c|}{ Source } & DF & $\begin{array}{c}\text { Chi- } \\
\text { Square }\end{array}$ & $\begin{array}{c}\text { P-Value } \\
\text { (Pr > ChiSq) }\end{array}$ \\
\hline YearLevel & 10 & 40.62 & $<.0001$ \\
Region & 2 & 4.18 & 0.124 \\
\hline
\end{tabular}


A. R.8: Estimates and Odds Ratio for Year Level and Region in 2004

\begin{tabular}{|l|l|r|r|r|r|}
\hline \multicolumn{7}{|c|}{ Analysis Of GEE Parameter Estimates } \\
\cline { 1 - 4 } Parameter & Level & Estimate & $\begin{array}{c}\text { Standard } \\
\text { Error }\end{array}$ & $\begin{array}{c}\text { P-Value } \\
(\text { Pr }>|\mathbf{Z}| \mathbf{)}\end{array}$ & $\begin{array}{c}\text { Odds } \\
\text { Ratio }\end{array}$ \\
\hline Intercept & & -3.1673 & 0.1873 & $<.0001$ & \\
YearLevel & Yr10 & 0.6816 & 0.2222 & 0.0022 & 1.9770 \\
YearLevel & Yr11 & 0.7142 & 0.3193 & 0.0253 & 2.0426 \\
YearLevel & Yr12 & 0.6625 & 0.3807 & 0.0818 & 1.9396 \\
YearLevel & Yr13 & 0.7806 & 0.3963 & 0.0489 & 2.1828 \\
YearLevel & Yr2 & 0.5554 & 0.1912 & 0.0037 & 1.7426 \\
YearLevel & Yr3 & -0.1037 & 0.1805 & 0.5658 & 0.9015 \\
YearLevel & Yr4 & -0.357 & 0.2011 & 0.0758 & 0.6998 \\
YearLevel & Yr5 & 0.0075 & 0.1828 & 0.9671 & 1.0075 \\
YearLevel & Yr6 & -0.0419 & 0.1818 & 0.8178 & 0.9590 \\
YearLevel & Yr7 & 0.0753 & 0.189 & 0.6904 & 1.0782 \\
YearLevel & Yr8 & 0 & 0 &. & 1.0000 \\
Region & Apia Urban & -0.122 & 0.2362 & 0.6054 & 0.8851 \\
Region & Rest of Upolu & 0.2977 & 0.1647 & 0.0707 & 1.3468 \\
Region & Savaii & 0 & 0 &. & 1.0000 \\
\hline
\end{tabular}

Score Statistics For Type 3 GEE Analysis

\begin{tabular}{|l|r|r|r|}
\hline \multicolumn{1}{|c|}{ Source } & DF & $\begin{array}{c}\text { Chi- } \\
\text { Square }\end{array}$ & $\begin{array}{c}\text { P-Value } \\
(\mathbf{P r}>\mathbf{C h i S q})\end{array}$ \\
\hline YearLevel & 10 & 34.52 & 0.0002 \\
Region & 2 & 5.56 & 0.0619 \\
\hline
\end{tabular}


A. R.9: Estimates and Odds Ratio for Year Level and Region in 2005

\begin{tabular}{|l|l|r|r|r|r|}
\hline \multicolumn{7}{|c|}{ Analysis Of GEE Parameter Estimates } & \multirow{2}{*}{} \\
\cline { 1 - 4 } Parameter & Level & Estimate & $\begin{array}{c}\text { Standard } \\
\text { Error }\end{array}$ & $\begin{array}{c}\text { P-Value } \\
(\mathbf{P r}>|\mathbf{Z}|)\end{array}$ & $\begin{array}{l}\text { Odds } \\
\text { Ratio }\end{array}$ \\
\hline Intercept & & -3.0848 & 0.1687 & $<.0001$ & \\
YearLevel & Yr10 & 0.041 & 0.3425 & 0.9048 & 1.0419 \\
YearLevel & Yr11 & 0.3759 & 0.3633 & 0.3008 & 1.4563 \\
YearLevel & Yr12 & 0.0292 & 0.4388 & 0.947 & 1.0296 \\
YearLevel & Yr13 & 0.9612 & 0.4224 & 0.0229 & 2.6148 \\
YearLevel & Yr2 & 0.5156 & 0.1773 & 0.0036 & 1.6746 \\
YearLevel & Yr3 & -0.0225 & 0.1693 & 0.8945 & 0.9778 \\
YearLevel & Yr4 & 0.0058 & 0.1419 & 0.9674 & 1.0058 \\
YearLevel & Yr5 & 0.036 & 0.1245 & 0.7724 & 1.0367 \\
YearLevel & Yr6 & 0.0867 & 0.1434 & 0.5454 & 1.0906 \\
YearLevel & Yr7 & 0.1394 & 0.1366 & 0.3075 & 1.1496 \\
YearLevel & Yr8 & 0 & 0 &. & 1.0000 \\
Region & Apia Urban & 0.3222 & 0.2129 & 0.1302 & 1.3802 \\
Region & Rest of Upolu & 0.3087 & 0.1692 & 0.0682 & 1.3617 \\
Region & Savaii & 0 & 0 &. & 1.0000 \\
\hline
\end{tabular}

Score Statistics For Type 3 GEE Analysis

\begin{tabular}{|l|r|r|r|}
\hline \multicolumn{1}{|c|}{ Source } & DF & $\begin{array}{c}\text { Chi- } \\
\text { Square }\end{array}$ & $\begin{array}{c}\text { P-Value } \\
\text { (Pr }>\text { ChiSq) }\end{array}$ \\
\hline YearLevel & 10 & 19.17 & 0.0382 \\
Region & 2 & 4.03 & 0.1335 \\
\hline
\end{tabular}


A. SL.1: Estimates and Odds Ratio for Year Level and School Level in 1996

\begin{tabular}{|c|c|c|c|c|c|}
\hline \multicolumn{5}{|c|}{ Analysis Of GEE Parameter Estimates } & \multirow[b]{3}{*}{$\begin{array}{l}\text { Odds } \\
\text { Ratio }\end{array}$} \\
\hline \multicolumn{5}{|c|}{ Empirical Standard Error Estimates } & \\
\hline Parameter & Level & Estimate & $\begin{array}{l}\text { Standard } \\
\text { Error }\end{array}$ & $\begin{array}{l}\text { P-Value } \\
(\mathrm{Pr}>|\mathrm{Z}|)\end{array}$ & \\
\hline Intercept & & -2.2599 & 0.326 & $<.0001$ & \\
\hline YearLevel & Yr10 & 0.1296 & 0.3076 & 0.6736 & 1.1384 \\
\hline YearLevel & Yr11 & -0.4703 & 0.4829 & 0.3302 & 0.6248 \\
\hline YearLevel & Yr12 & -0.1123 & 0.4451 & 0.8008 & 0.8938 \\
\hline YearLevel & Yr13 & 0.9213 & 0.7494 & 0.2189 & 2.5126 \\
\hline YearLevel & Yr2 & 1.1814 & 0.2416 & $<.0001$ & 3.2589 \\
\hline YearLevel & Yr3 & 0.4362 & 0.2036 & 0.0322 & 1.5468 \\
\hline YearLevel & Yr4 & 0.2823 & 0.2395 & 0.2386 & 1.3262 \\
\hline YearLevel & Yr5 & 0.484 & 0.2344 & 0.039 & 1.6226 \\
\hline YearLevel & Yr6 & 0.7654 & 0.2298 & 0.0009 & 2.1499 \\
\hline YearLevel & Yr7 & 0.5597 & 0.2173 & 0.01 & 1.7501 \\
\hline YearLevel & Yr8 & 0 & 0 & & 1.0000 \\
\hline SchoolLevel & Primary & -1.1343 & 0.3305 & 0.0006 & 0.3216 \\
\hline SchoolLevel & Primary/Secondary & 0.1536 & 0.2038 & 0.451 & 1.1660 \\
\hline SchoolLevel & Secondary & 0 & 0 & & 1.0000 \\
\hline
\end{tabular}

\begin{tabular}{|c|c|c|c|}
\hline \multicolumn{4}{|c|}{ Score Statistics For Type 3 GEE Analysis } \\
\hline Source & DF & $\begin{array}{c}\text { Chi- } \\
\text { Square }\end{array}$ & $\begin{array}{c}\text { P-Value } \\
(\mathrm{Pr}>\mathrm{ChiSq})\end{array}$ \\
\hline YearLevel & & 35.59 & $<.0001$ \\
\hline SchoolLevel & & 1.93 & 0.3805 \\
\hline
\end{tabular}


A. SL.2: Estimates and Odds Ratio for Year Level and School Level in 1997

\begin{tabular}{|c|c|c|c|c|c|}
\hline \multicolumn{5}{|c|}{ Analysis Of GEE Parameter Estimates } & \multirow[b]{3}{*}{$\begin{array}{l}\text { Odds } \\
\text { Ratio }\end{array}$} \\
\hline \multicolumn{5}{|c|}{ Empirical Standard Error Estimates } & \\
\hline Parameter & Level & Estimate & $\begin{array}{l}\text { Standard } \\
\text { Error }\end{array}$ & $\begin{array}{l}\text { P-Value } \\
(\operatorname{Pr}>|Z|)\end{array}$ & \\
\hline Intercept & & -5.4705 & 1.599 & 0.0006 & \\
\hline YearLevel & Yr10 & 3.1091 & 1.5901 & 0.0505 & 22.4009 \\
\hline YearLevel & Yr11 & 2.6485 & 1.6246 & 0.103 & 14.1328 \\
\hline YearLevel & Yr12 & 3.1808 & 1.6158 & 0.049 & 24.0660 \\
\hline YearLevel & Yr13 & 5.0508 & 1.6289 & 0.0019 & 156.1473 \\
\hline YearLevel & Yr2 & 1.4227 & 0.2656 & $<.0001$ & 4.1483 \\
\hline YearLevel & Yr3 & 0.5457 & 0.2567 & 0.0335 & 1.7258 \\
\hline YearLevel & Yr4 & 0.6595 & 0.2491 & 0.0081 & 1.9338 \\
\hline YearLevel & Yr5 & 0.6673 & 0.2792 & 0.0168 & 1.9490 \\
\hline YearLevel & Yr6 & 0.5446 & 0.2548 & 0.0326 & 1.7239 \\
\hline YearLevel & Yr7 & 0.8982 & 0.2556 & 0.0004 & 2.4552 \\
\hline YearLevel & Yr8 & 0 & 0 & & 1.0000 \\
\hline SchoolLevel & Primary & 1.7308 & 1.5959 & 0.2781 & 5.6452 \\
\hline SchoolLevel & Primary/Secondary & 0.471 & 0.2953 & 0.1107 & 1.6016 \\
\hline SchoolLevel & Secondary & 0 & 0 & & 1.0000 \\
\hline
\end{tabular}

\begin{tabular}{|c|c|c|c|}
\hline \multicolumn{4}{|c|}{ Score Statistics For Type 3 GEE Analysis } \\
\hline Source & DF & $\begin{array}{l}\text { Chi- } \\
\text { Square }\end{array}$ & $\begin{array}{c}\text { P-Value } \\
(\mathrm{Pr}>\mathrm{ChiSq})\end{array}$ \\
\hline YearLevel & 10 & 43.64 & $<.0001$ \\
\hline SchoolLevel & 2 & 2.61 & 0.2718 \\
\hline
\end{tabular}


A. SL.3: Estimates and Odds Ratio for Year Level and School Level in 1998

\begin{tabular}{|l|lrrrr|r|}
\hline \multicolumn{5}{|c|}{ Analysis Of GEE Parameter Estimates } \\
\cline { 1 - 4 } Parameter & Level & Estimate & $\begin{array}{c}\text { Standard } \\
\text { Error }\end{array}$ & $\begin{array}{c}\text { P-Value } \\
(\text { Pr }>|Z|)\end{array}$ & $\begin{array}{l}\text { Odds } \\
\text { Ratio }\end{array}$ \\
\hline Intercept & & -2.2795 & 0.6184 & 0.0002 & \\
YearLevel & Yr10 & -0.2254 & 0.6137 & 0.7134 & 0.7982 \\
YearLevel & Yr11 & -0.9848 & 0.8258 & 0.2331 & 0.3735 \\
YearLevel & Yr12 & 0.5199 & 0.5682 & 0.3602 & 1.6819 \\
YearLevel & Yr13 & 0.1742 & 0.6938 & 0.8017 & 1.1903 \\
YearLevel & Yr2 & 1.4392 & 0.2628 & $<.0001$ & 4.2173 \\
YearLevel & Yr3 & 0.6703 & 0.2708 & 0.0133 & 1.9548 \\
YearLevel & Yr4 & 0.5429 & 0.243 & 0.0255 & 1.7210 \\
YearLevel & Yr5 & 0.8955 & 0.2668 & 0.0008 & 2.4486 \\
YearLevel & Yr6 & 0.6117 & 0.2565 & 0.0171 & 1.8436 \\
YearLevel & Yr7 & 0.7727 & 0.2542 & 0.0024 & 2.1656 \\
YearLevel & Yr8 & 0 & 0 &. & 1.0000 \\
SchoolLevel & Primary & -1.5447 & 0.6087 & 0.0112 & 0.2134 \\
SchoolLevel & Primary/Secondary & -0.5192 & 0.5066 & 0.3054 & 0.5950 \\
SchoolLevel & Secondary & 0 & 0 &. & 1.0000 \\
\hline
\end{tabular}

\begin{tabular}{|l|rrrr|}
\hline \multicolumn{4}{|c|}{ Score Statistics For Type 3 GEE Analysis } \\
\hline \multicolumn{1}{|c|}{ Source } & DF & $\begin{array}{c}\text { Chi- } \\
\text { Square }\end{array}$ & $\begin{array}{c}\text { P-Value } \\
\text { (Pr > ChiSq) }\end{array}$ \\
\hline YearLevel & & 10 & 33.39 & 0.0002 \\
SchoolLevel & & 2 & 1.57 & 0.456 \\
\hline
\end{tabular}


A. SL.4: Estimates and Odds Ratio for Year Level and School Level in 1999

\begin{tabular}{|c|c|c|c|c|c|}
\hline \multicolumn{5}{|c|}{ Analysis Of GEE Parameter Estimates } & \multirow[b]{3}{*}{$\begin{array}{l}\text { Odds } \\
\text { Ratio }\end{array}$} \\
\hline \multicolumn{5}{|c|}{ Empirical Standard Error Estimates } & \\
\hline Parameter & Level & Estimate & $\begin{array}{c}\text { Standard } \\
\text { Error }\end{array}$ & $\begin{array}{c}\text { P-Value } \\
(\mathrm{Pr}>|\mathrm{Z}|)\end{array}$ & \\
\hline Intercept & & -1.6665 & 1.1163 & 0.1355 & \\
\hline YearLevel & Yr10 & -0.7785 & 1.1472 & 0.4974 & 0.4591 \\
\hline YearLevel & Yr11 & -1.2303 & 1.1836 & 0.2986 & 0.2922 \\
\hline YearLevel & Yr12 & -0.1326 & 1.0773 & 0.902 & 0.8758 \\
\hline YearLevel & Yr13 & -0.7096 & 1.1264 & 0.5287 & 0.4918 \\
\hline YearLevel & Yr2 & 1.3448 & 0.2521 & $<.0001$ & 3.8374 \\
\hline YearLevel & Yr3 & 0.6881 & 0.263 & 0.0089 & 1.9899 \\
\hline YearLevel & Yr4 & 0.7798 & 0.2354 & 0.0009 & 2.1810 \\
\hline YearLevel & Yr5 & 0.7789 & 0.2631 & 0.0031 & 2.1791 \\
\hline YearLevel & Yr6 & 0.656 & 0.2464 & 0.0078 & 1.9271 \\
\hline YearLevel & Yr7 & 1.0539 & 0.2428 & $<.0001$ & 2.8688 \\
\hline YearLevel & Yr8 & 0 & 0 & & 1.0000 \\
\hline SchoolLevel & Primary & -2.2254 & 1.104 & 0.0438 & 0.1080 \\
\hline SchoolLevel & Primary/Secondary & -1.1477 & 0.8433 & 0.1735 & 0.3174 \\
\hline SchoolLevel & Secondary & 0 & 0 & & 1.0000 \\
\hline
\end{tabular}

\begin{tabular}{|c|c|c|c|}
\hline \multicolumn{4}{|c|}{ Score Statistics For Type 3 GEE Analysis } \\
\hline Source & DF & $\begin{array}{l}\text { Chi- } \\
\text { Square }\end{array}$ & $\begin{array}{c}\text { P-Value } \\
(\mathrm{Pr}>\mathrm{ChiSq})\end{array}$ \\
\hline YearLevel & 10 & 38.03 & $<.0001$ \\
\hline SchoolLevel & 2 & 2.6 & 0.2731 \\
\hline
\end{tabular}


A. SL.5: Estimates and Odds Ratio for Year Level and School Level in 2000

\begin{tabular}{|c|c|c|c|c|c|}
\hline \multicolumn{5}{|c|}{ Analysis Of GEE Parameter Estimates } & \multirow[b]{3}{*}{$\begin{array}{l}\text { Odds } \\
\text { Ratio }\end{array}$} \\
\hline \multicolumn{5}{|c|}{ Empirical Standard Error Estimates } & \\
\hline Parameter & Level & Estimate & $\begin{array}{l}\text { Standard } \\
\text { Error }\end{array}$ & $\begin{array}{c}\text { P-Value } \\
(\operatorname{Pr}>|Z|)\end{array}$ & \\
\hline Intercept & & -2.5372 & 0.7263 & 0.0005 & \\
\hline YearLevel & Yr10 & 0.3776 & 0.7179 & 0.5989 & 1.4588 \\
\hline YearLevel & Yr11 & -0.1526 & 0.7729 & 0.8435 & 0.8585 \\
\hline YearLevel & Yr12 & 0.7535 & 0.7451 & 0.3119 & 2.1244 \\
\hline YearLevel & Yr13 & -0.1922 & 0.8386 & 0.8187 & 0.8251 \\
\hline YearLevel & Yr2 & 1.1742 & 0.2256 & $<.0001$ & 3.2356 \\
\hline YearLevel & Yr3 & 0.3746 & 0.2263 & 0.0979 & 1.4544 \\
\hline YearLevel & Yr4 & 0.4673 & 0.2147 & 0.0295 & 1.5957 \\
\hline YearLevel & Yr5 & 0.655 & 0.2587 & 0.0113 & 1.9251 \\
\hline YearLevel & Yr6 & 0.64 & 0.216 & 0.003 & 1.8965 \\
\hline YearLevel & Yr7 & 0.6592 & 0.2254 & 0.0034 & 1.9332 \\
\hline YearLevel & Yr8 & 0 & 0 & & 1.0000 \\
\hline SchoolLevel & Primary & -0.8574 & 0.7122 & 0.2286 & 0.4243 \\
\hline SchoolLevel & Primary/Secondary & -0.55 & 0.4278 & 0.1986 & 0.5769 \\
\hline SchoolLevel & Secondary & 0 & 0 & & 1.0000 \\
\hline
\end{tabular}

\begin{tabular}{|c|c|c|c|}
\hline \multicolumn{4}{|c|}{ Score Statistics For Type 3 GEE Analysis } \\
\hline Source & DF & $\begin{array}{l}\text { Chi- } \\
\text { Square }\end{array}$ & $\begin{array}{c}\text { P-Value } \\
(\mathrm{Pr}>\mathrm{ChiSq})\end{array}$ \\
\hline YearLevel & 10 & 36.71 & $<.0001$ \\
\hline SchoolLevel & 2 & 1.57 & 0.4561 \\
\hline
\end{tabular}


A. SL.6: Estimates and Odds Ratio for Year Level and School Level in 2001

\begin{tabular}{|c|c|c|c|c|c|}
\hline \multicolumn{5}{|c|}{ Analysis Of GEE Parameter Estimates } & \multirow[b]{3}{*}{$\begin{array}{l}\text { Odds } \\
\text { Ratio }\end{array}$} \\
\hline \multicolumn{5}{|c|}{ Empirical Standard Error Estimates } & \\
\hline Parameter & Level & Estimate & $\begin{array}{c}\text { Standard } \\
\text { Error }\end{array}$ & $\begin{array}{l}\text { P-Value } \\
(\operatorname{Pr}>|\mathrm{Z}|)\end{array}$ & \\
\hline Intercept & & -2.7656 & 0.4628 & $<.0001$ & \\
\hline YearLevel & Yr10 & 0.3861 & 0.4559 & 0.3971 & 1.4712 \\
\hline YearLevel & Yr11 & 0.3841 & 0.5319 & 0.4702 & 1.4683 \\
\hline YearLevel & Yr12 & 0.598 & 0.5178 & 0.2481 & 1.8185 \\
\hline YearLevel & Yr13 & 0.332 & 0.5389 & 0.5378 & 1.3938 \\
\hline YearLevel & Yr2 & 0.8469 & 0.2044 & $<.0001$ & 2.3324 \\
\hline YearLevel & Yr3 & 0.3562 & 0.2506 & 0.1551 & 1.4279 \\
\hline YearLevel & Yr4 & 0.2507 & 0.1884 & 0.1832 & 1.2849 \\
\hline YearLevel & Yr5 & 0.2764 & 0.2091 & 0.1862 & 1.3184 \\
\hline YearLevel & Yr6 & 0.1337 & 0.203 & 0.5104 & 1.1430 \\
\hline YearLevel & Yr7 & 0.4854 & 0.1837 & 0.0082 & 1.6248 \\
\hline YearLevel & Yr8 & 0 & 0 & & 1.0000 \\
\hline SchoolLevel & Primary & -0.5947 & 0.4544 & 0.1906 & 0.5517 \\
\hline SchoolLevel & Primary/Secondary & 0.0953 & 0.4323 & 0.8256 & 1.1000 \\
\hline SchoolLevel & Secondary & 0 & 0 & & 1.0000 \\
\hline
\end{tabular}

\begin{tabular}{|c|c|c|c|}
\hline \multicolumn{4}{|c|}{ Score Statistics For Type 3 GEE Analysis } \\
\hline Source & DF & $\begin{array}{l}\text { Chi- } \\
\text { Square }\end{array}$ & $\begin{array}{c}\text { P-Value } \\
\text { (Pr }>\text { ChiSq) }\end{array}$ \\
\hline YearLevel & 10 & 21.39 & 0.0185 \\
\hline SchoolLevel & 2 & 1.95 & 0.3766 \\
\hline
\end{tabular}


A. SL.7: Estimates and Odds Ratio for Year Level and School Level in 2002

\begin{tabular}{|l|l|r|r|r|r|}
\hline \multicolumn{5}{|c|}{ Analysis Of GEE Parameter Estimates } \\
\cline { 1 - 4 } Parameter & Level & Estimate & $\begin{array}{c}\text { Standard } \\
\text { Error }\end{array}$ & $\begin{array}{r}\text { P-Value } \\
(\text { Pr }>|Z|)\end{array}$ & $\begin{array}{l}\text { Odds } \\
\text { Ratio }\end{array}$ \\
\hline Intercept & & -3.1906 & 0.5075 & $<.0001$ & \\
YearLevel & Yr10 & 0.7036 & 0.5359 & 0.1892 & 2.0210 \\
YearLevel & Yr11 & 0.9579 & 0.5895 & 0.1041 & 2.6062 \\
YearLevel & Yr12 & 0.6909 & 0.5793 & 0.233 & 1.9955 \\
YearLevel & Yr13 & 0.612 & 0.5519 & 0.2675 & 1.8441 \\
YearLevel & Yr2 & 0.7775 & 0.196 & $<.0001$ & 2.1760 \\
YearLevel & Yr3 & 0.0582 & 0.2576 & 0.8214 & 1.0599 \\
YearLevel & Yr4 & -0.1521 & 0.1993 & 0.4454 & 0.8589 \\
YearLevel & Yr5 & 0.297 & 0.2002 & 0.138 & 1.3458 \\
YearLevel & Yr6 & 0.017 & 0.1989 & 0.932 & 1.0171 \\
YearLevel & Yr7 & 0.1994 & 0.1986 & 0.3154 & 1.2207 \\
YearLevel & Yr8 & 0 & 0 &. & 1.0000 \\
SchoolLevel & Primary & -0.1372 & 0.5208 & 0.7922 & 0.8718 \\
SchoolLevel & Primary/Secondary & -1.2637 & 0.6609 & 0.0559 & 0.2826 \\
SchoolLevel & Secondary & 0 & 0 &. & 1.0000 \\
\hline
\end{tabular}

\begin{tabular}{|c|c|c|c|}
\hline \multicolumn{4}{|c|}{ Score Statistics For Type 3 GEE Analysis } \\
\hline Source & DF & $\begin{array}{l}\text { Chi- } \\
\text { Square }\end{array}$ & $\begin{array}{c}\text { P-Value } \\
(\mathrm{Pr}>\mathrm{ChiSq})\end{array}$ \\
\hline YearLevel & 10 & 27.85 & 0.0019 \\
\hline SchoolLevel & 2 & 2.76 & 0.252 \\
\hline
\end{tabular}


A. SL.8: Estimates and Odds Ratio for Year Level and School Level in 2003

\begin{tabular}{|c|c|c|c|c|c|}
\hline \multicolumn{5}{|c|}{ Analysis Of GEE Parameter Estimates } & \multirow[b]{3}{*}{$\begin{array}{l}\text { Odds } \\
\text { Ratio }\end{array}$} \\
\hline \multicolumn{5}{|c|}{ Empirical Standard Error Estimates } & \\
\hline Parameter & Level & Estimate & $\begin{array}{c}\text { Standard } \\
\text { Error }\end{array}$ & $\begin{array}{l}\text { P-Value } \\
(\operatorname{Pr}>|Z|)\end{array}$ & \\
\hline Intercept & & -1.9176 & 0.6069 & 0.0016 & \\
\hline YearLevel & Yr10 & -0.9137 & 0.626 & 0.1444 & 0.4010 \\
\hline YearLevel & Yr11 & -0.4565 & 0.665 & 0.4925 & 0.6335 \\
\hline YearLevel & Yr12 & -0.7559 & 0.7285 & 0.2994 & 0.4696 \\
\hline YearLevel & Yr13 & -0.3798 & 0.7118 & 0.5936 & 0.6840 \\
\hline YearLevel & Yr2 & 0.5952 & 0.2279 & 0.009 & 1.8134 \\
\hline YearLevel & Yr3 & 0.2904 & 0.2184 & 0.1835 & 1.3370 \\
\hline YearLevel & Yr4 & -0.2451 & 0.2376 & 0.3021 & 0.7826 \\
\hline YearLevel & Yr5 & 0.2842 & 0.2287 & 0.2139 & 1.3287 \\
\hline YearLevel & Yr6 & -0.0706 & 0.2903 & 0.8079 & 0.9318 \\
\hline YearLevel & Yr7 & 0.4827 & 0.2102 & 0.0216 & 1.6204 \\
\hline YearLevel & Yr8 & 0 & 0 & & 1.0000 \\
\hline SchoolLevel & Primary & -1.5477 & 0.6396 & 0.0155 & 0.2127 \\
\hline SchoolLevel & Primary/Secondary & -1.635 & 0.7411 & 0.0274 & 0.1950 \\
\hline SchoolLevel & Secondary & 0 & 0 & & 1.0000 \\
\hline
\end{tabular}

\begin{tabular}{|c|c|c|c|}
\hline \multicolumn{4}{|c|}{ Score Statistics For Type 3 GEE Analysis } \\
\hline Source & DF & $\begin{array}{l}\text { Chi- } \\
\text { Square }\end{array}$ & $\begin{array}{c}\text { P-Value } \\
(\mathrm{Pr}>\mathrm{ChiSq})\end{array}$ \\
\hline YearLevel & 10 & 25.33 & 0.0047 \\
\hline SchoolLevel & 2 & 3.71 & 0.1567 \\
\hline
\end{tabular}


A. SL.9: Estimates and Odds Ratio for Year Level and School Level in 2004

\begin{tabular}{|c|c|c|c|c|c|}
\hline \multicolumn{5}{|c|}{ Analysis Of GEE Parameter Estimates } & \multirow[b]{3}{*}{$\begin{array}{l}\text { Odds } \\
\text { Ratio }\end{array}$} \\
\hline \multicolumn{5}{|c|}{ Empirical Standard Error Estimates } & \\
\hline Parameter & Level & Estimate & $\begin{array}{c}\text { Standard } \\
\text { Error }\end{array}$ & $\begin{array}{l}\text { P-Value } \\
(\operatorname{Pr}>|\mathrm{Z}|)\end{array}$ & \\
\hline Intercept & & -1.5587 & 0.6356 & 0.0142 & \\
\hline YearLevel & Yr10 & -0.7889 & 0.6361 & 0.2149 & 0.4543 \\
\hline YearLevel & Yr11 & -0.75 & 0.6848 & 0.2734 & 0.4724 \\
\hline YearLevel & Yr12 & -0.811 & 0.7002 & 0.2467 & 0.4444 \\
\hline YearLevel & Yr13 & -0.6686 & 0.6817 & 0.3267 & 0.5124 \\
\hline YearLevel & Yr2 & 0.5549 & 0.1929 & 0.004 & 1.7418 \\
\hline YearLevel & Yr3 & -0.1041 & 0.1831 & 0.5698 & 0.9011 \\
\hline YearLevel & Yr4 & -0.3608 & 0.2022 & 0.0743 & 0.6971 \\
\hline YearLevel & Yr5 & 0.0048 & 0.1857 & 0.9795 & 1.0048 \\
\hline YearLevel & Yr6 & -0.0413 & 0.1845 & 0.8231 & 0.9595 \\
\hline YearLevel & Yr7 & 0.0722 & 0.1911 & 0.7055 & 1.0749 \\
\hline YearLevel & Yr8 & 0 & 0 & & 1.0000 \\
\hline SchoolLevel & Primary & -1.5099 & 0.6559 & 0.0213 & 0.2209 \\
\hline SchoolLevel & Primary/Secondary & -2.3627 & 0.6769 & 0.0005 & 0.0942 \\
\hline SchoolLevel & Secondary & 0 & 0 & 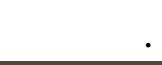 & 1.0000 \\
\hline
\end{tabular}

\begin{tabular}{|c|c|c|c|}
\hline \multicolumn{4}{|c|}{ Score Statistics For Type 3 GEE Analysis } \\
\hline Source & DF & $\begin{array}{c}\text { Chi- } \\
\text { Square }\end{array}$ & $\begin{array}{c}\text { P-Value } \\
(\mathrm{Pr}>\mathrm{ChiSq})\end{array}$ \\
\hline YearLevel & 10 & 24.35 & 0.0067 \\
\hline SchoolLevel & 2 & 4.12 & 0.1275 \\
\hline
\end{tabular}


A. SL.10: Estimates and Odds Ratio for Year Level and School Level in 2005

\begin{tabular}{|c|c|c|c|c|c|}
\hline \multicolumn{5}{|c|}{ Analysis Of GEE Parameter Estimates } & \multirow[b]{3}{*}{$\begin{array}{l}\text { Odds } \\
\text { ratio }\end{array}$} \\
\hline \multicolumn{5}{|c|}{ Empirical Standard Error Estimates } & \\
\hline Parameter & Level & Estimate & $\begin{array}{l}\text { Standard } \\
\text { Error }\end{array}$ & $\begin{array}{c}\text { P-Value } \\
(\operatorname{Pr}>|Z|)\end{array}$ & \\
\hline Intercept & & -1.3226 & 0.7111 & 0.0629 & \\
\hline YearLevel & Yr10 & -1.4035 & 0.725 & 0.0529 & 0.2457 \\
\hline YearLevel & Yr11 & -1.0727 & 0.7663 & 0.1616 & 0.3421 \\
\hline YearLevel & Yr12 & -1.4136 & 0.8002 & 0.0773 & 0.2433 \\
\hline YearLevel & Yr13 & -0.4281 & 0.688 & 0.5338 & 0.6517 \\
\hline YearLevel & Yr2 & 0.5817 & 0.1817 & 0.0014 & 1.7891 \\
\hline YearLevel & Yr3 & 0.0323 & 0.1734 & 0.8523 & 1.0328 \\
\hline YearLevel & Yr4 & 0.0616 & 0.1438 & 0.6681 & 1.0635 \\
\hline YearLevel & Yr5 & 0.0898 & 0.1205 & 0.456 & 1.0940 \\
\hline YearLevel & Yr6 & 0.1477 & 0.1437 & 0.3039 & 1.1592 \\
\hline YearLevel & Yr7 & 0.2016 & 0.1376 & 0.1428 & 1.2234 \\
\hline YearLevel & Yr8 & 0 & 0 & & 1.0000 \\
\hline SchoolLevel & Primary & -1.6447 & 0.7085 & 0.0203 & 0.1931 \\
\hline SchoolLevel & Primary/Secondary & -0.6924 & 0.583 & 0.235 & 0.5004 \\
\hline SchoolLevel & Secondary & 0 & 0 & & 1.0000 \\
\hline
\end{tabular}

\begin{tabular}{|l|rrrr|}
\hline \multicolumn{4}{|c|}{ Score Statistics For Type 3 GEE Analysis } \\
\hline \multicolumn{1}{|c|}{ Source } & DF & $\begin{array}{c}\text { Chi- } \\
\text { Square }\end{array}$ & $\begin{array}{r}\text { P-Value } \\
(\mathbf{P r}>\text { ChiSq) }\end{array}$ \\
\hline YearLevel & \multicolumn{10}{c}{20.93} & 0.0216 \\
SchoolLevel & & 2 & 3.12 & 0.2106 \\
\hline
\end{tabular}


A. SL.11: Estimates and Odds Ratio for Year Level and School Level in 2006

\begin{tabular}{|c|c|c|c|c|c|}
\hline \multicolumn{5}{|c|}{ Analysis Of GEE Parameter Estimates } & \multirow[b]{3}{*}{$\begin{array}{l}\text { Odds } \\
\text { Ratio }\end{array}$} \\
\hline \multicolumn{5}{|c|}{ Empirical Standard Error Estimates } & \\
\hline Parameter & Level & Estimate & $\begin{array}{l}\text { Standard } \\
\text { Error }\end{array}$ & $\begin{array}{c}\text { P-Value } \\
(\operatorname{Pr}>|Z|)\end{array}$ & \\
\hline Intercept & & -2.9886 & 0.3368 & $<.0001$ & \\
\hline YearLevel & Yr10 & 0.5278 & 0.3212 & 0.1004 & 1.6952 \\
\hline YearLevel & Yr11 & 0.5774 & 0.4325 & 0.1818 & 1.7814 \\
\hline YearLevel & Yr12 & 0.3299 & 0.4969 & 0.5068 & 1.3908 \\
\hline YearLevel & Yr13 & 1.8426 & 0.3614 & $<.0001$ & 6.3129 \\
\hline YearLevel & Yr2 & 0.4677 & 0.1909 & 0.0143 & 1.5963 \\
\hline YearLevel & Yr3 & 0.0954 & 0.1952 & 0.6252 & 1.1001 \\
\hline YearLevel & Yr4 & -0.3536 & 0.2033 & 0.082 & 0.7022 \\
\hline YearLevel & Yr5 & -0.2907 & 0.2034 & 0.1529 & 0.7477 \\
\hline YearLevel & Yr6 & -0.0243 & 0.1961 & 0.9016 & 0.9760 \\
\hline YearLevel & Yr7 & -0.1818 & 0.172 & 0.2904 & 0.8338 \\
\hline YearLevel & Yr8 & 0 & 0 & & 1.0000 \\
\hline SchoolLevel & Primary & 0.1384 & 0.3459 & 0.6889 & 1.1484 \\
\hline SchoolLevel & Primary/Secondary & -0.8808 & 0.4329 & 0.0419 & 0.4145 \\
\hline SchoolLevel & Secondary & 0 & 0 & & 1.0000 \\
\hline
\end{tabular}

\begin{tabular}{|l|rrrr|}
\hline \multicolumn{4}{|c|}{ Score Statistics For Type 3 GEE Analysis } \\
\hline \multicolumn{1}{|c|}{ Source } & DF & \multicolumn{1}{c|}{$\begin{array}{c}\text { Chi- } \\
\text { Square }\end{array}$} & $\begin{array}{c}\text { P-Value } \\
\text { (Pr > ChiSq) }\end{array}$ \\
\hline YearLevel & & 10 & 42.34 & $<.0001$ \\
SchoolLevel & & 2 & 2.47 & 0.2915 \\
\hline
\end{tabular}


A. SS.1: Estimates and Odds Ratio for Year Level and School Status in 1995

\begin{tabular}{|c|c|c|c|c|c|}
\hline \multicolumn{5}{|c|}{ Analysis Of GEE Parameter Estimates } & \multirow[b]{3}{*}{$\begin{array}{l}\text { Odds } \\
\text { Ratio }\end{array}$} \\
\hline \multicolumn{5}{|c|}{ Empirical Standard Error Estimates } & \\
\hline Parameter & Level & Estimate & $\begin{array}{l}\text { Standard } \\
\text { Error }\end{array}$ & $\begin{array}{l}\text { P-Value } \\
(\operatorname{Pr}>|Z|)\end{array}$ & \\
\hline Intercept & & -4.0465 & 0.7026 & $<.0001$ & \\
\hline YearLevel & Yr10 & 0.8834 & 0.2681 & 0.001 & 2.4191 \\
\hline YearLevel & Yr11 & 0.3905 & 0.4954 & 0.4305 & 1.4777 \\
\hline YearLevel & Yr12 & -0.0881 & 1.1435 & 0.9386 & 0.9157 \\
\hline YearLevel & Yr13 & 1.7145 & 0.8223 & 0.0371 & 5.5539 \\
\hline YearLevel & Yr2 & 0.8934 & 0.2574 & 0.0005 & 2.4434 \\
\hline YearLevel & Yr3 & 0.0524 & 0.2607 & 0.8406 & 1.0538 \\
\hline YearLevel & Yr4 & 0.0241 & 0.2567 & 0.9251 & 1.0244 \\
\hline YearLevel & Yr5 & 0.2658 & 0.2411 & 0.2701 & 1.3045 \\
\hline YearLevel & Yr6 & 0.0612 & 0.2575 & 0.8122 & 1.0631 \\
\hline YearLevel & Yr7 & 0.2742 & 0.2397 & 0.2528 & 1.3155 \\
\hline YearLevel & Yr8 & 0 & 0 & & 1.0000 \\
\hline SchoolStatus & Government & 0.6169 & 0.6798 & 0.3641 & 1.8532 \\
\hline SchoolStatus & Mission & 0.6068 & 0.7305 & 0.4062 & 1.8346 \\
\hline SchoolStatus & Private & 0 & 0 & & 1.0000 \\
\hline
\end{tabular}

\begin{tabular}{|l|r|rr|}
\hline \multicolumn{4}{|c|}{ Score Statistics For Type 3 GEE Analysis } \\
\hline \multicolumn{1}{|c|}{ Source } & DF & $\begin{array}{c}\text { Chi- } \\
\text { Square }\end{array}$ & $\begin{array}{c}\text { P-Value } \\
\text { (Pr > ChiSq) }\end{array}$ \\
\hline YearLevel & \multicolumn{1}{c}{10} & 34.23 & 0.0002 \\
SchoolStatus & 2 & 0.76 & 0.6849 \\
\hline
\end{tabular}


A. SS.2: Estimates and Odds Ratio for Year Level and School Status in 1996

\begin{tabular}{|c|c|c|c|c|c|}
\hline \multicolumn{5}{|c|}{ Analysis Of GEE Parameter Estimates } & \multirow[b]{3}{*}{$\begin{array}{l}\text { Odds } \\
\text { Ratio }\end{array}$} \\
\hline \multicolumn{5}{|c|}{ Empirical Standard Error Estimates } & \\
\hline Parameter & Level & Estimate & $\begin{array}{l}\text { Standard } \\
\text { Error }\end{array}$ & $\begin{array}{c}\text { P-Value } \\
(\operatorname{Pr}>|\mathrm{Z}|)\end{array}$ & \\
\hline Intercept & & -3.742 & 0.5892 & $<.0001$ & \\
\hline YearLevel & Yr10 & 1.3217 & 0.2801 & $<.0001$ & 3.7498 \\
\hline YearLevel & Yr11 & 0.7385 & 0.4213 & 0.0796 & 2.0928 \\
\hline YearLevel & Yr12 & 1.2042 & 0.5335 & 0.024 & 3.3341 \\
\hline YearLevel & Yr13 & 2.1377 & 0.7845 & 0.0064 & 8.4799 \\
\hline YearLevel & Yr2 & 1.1706 & 0.2453 & $<.0001$ & 3.2239 \\
\hline YearLevel & Yr3 & 0.4271 & 0.2072 & 0.0393 & 1.5328 \\
\hline YearLevel & Yr4 & 0.2794 & 0.2422 & 0.2488 & 1.3223 \\
\hline YearLevel & Yr5 & 0.4781 & 0.2375 & 0.0441 & 1.6130 \\
\hline YearLevel & Yr6 & 0.756 & 0.2309 & 0.0011 & 2.1297 \\
\hline YearLevel & Yr7 & 0.5569 & 0.2205 & 0.0115 & 1.7453 \\
\hline YearLevel & Yr8 & 0 & 0 & & 1.0000 \\
\hline SchoolStatus & Government & 0.4019 & 0.558 & 0.4713 & 1.4947 \\
\hline SchoolStatus & Mission & 0.0188 & 0.614 & 0.9756 & 1.0190 \\
\hline SchoolStatus & Private & 0 & 0 & & 1.0000 \\
\hline
\end{tabular}

Score Statistics For Type 3 GEE Analysis

\begin{tabular}{|c|c|c|c|}
\hline Source & DF & $\begin{array}{c}\text { Chi- } \\
\text { Square }\end{array}$ & $\begin{array}{c}\text { P-Value } \\
(\mathrm{Pr}>\mathrm{ChiSq})\end{array}$ \\
\hline YearLevel & 10 & 45.98 & $<.0001$ \\
\hline SchoolStatus & 2 & 2.31 & 0.315 \\
\hline
\end{tabular}


A. SS.3: Estimates and Odds Ratio for Year Level and School Status in 1997

\begin{tabular}{|c|c|c|c|c|c|}
\hline \multicolumn{5}{|c|}{ Analysis Of GEE Parameter Estimates } & \multirow[b]{3}{*}{$\begin{array}{l}\text { Odds } \\
\text { Ratio }\end{array}$} \\
\hline \multicolumn{5}{|c|}{ Empirical Standard Error Estimates } & \\
\hline Parameter & Level & Estimate & $\begin{array}{l}\text { Standard } \\
\text { Error }\end{array}$ & $\begin{array}{l}\text { P-Value } \\
(\operatorname{Pr}>|Z|)\end{array}$ & \\
\hline Intercept & & -3.6461 & 0.9959 & 0.0003 & \\
\hline YearLevel & Yr10 & 1.5549 & 0.31 & $<.0001$ & 4.7346 \\
\hline YearLevel & Yr11 & 1.0938 & 0.4347 & 0.0119 & 2.9856 \\
\hline YearLevel & Yr12 & 1.7764 & 0.5119 & 0.0005 & 5.9085 \\
\hline YearLevel & Yr13 & 3.6109 & 0.474 & $<.0001$ & 36.9993 \\
\hline YearLevel & Yr2 & 1.4418 & 0.2696 & $<.0001$ & 4.2283 \\
\hline YearLevel & Yr3 & 0.558 & 0.2596 & 0.0316 & 1.7472 \\
\hline YearLevel & Yr4 & 0.6761 & 0.2517 & 0.0072 & 1.9662 \\
\hline YearLevel & Yr5 & 0.6803 & 0.2838 & 0.0165 & 1.9745 \\
\hline YearLevel & Yr6 & 0.5567 & 0.2581 & 0.031 & 1.7449 \\
\hline YearLevel & Yr7 & 0.9168 & 0.2582 & 0.0004 & 2.5013 \\
\hline YearLevel & Yr8 & 0 & 0 & & 1.0000 \\
\hline SchoolStatus & Government & -0.0435 & 0.9811 & 0.9646 & 0.9574 \\
\hline SchoolStatus & Mission & -0.8002 & 1.0325 & 0.4383 & 0.4492 \\
\hline SchoolStatus & Private & 0 & 0 & & 1.0000 \\
\hline
\end{tabular}

Score Statistics For Type 3 GEE Analysis

\begin{tabular}{|l|r|r|r|}
\hline \multicolumn{1}{|c|}{ Source } & DF & $\begin{array}{c}\text { Chi- } \\
\text { Square }\end{array}$ & $\begin{array}{c}\text { P-Value } \\
\text { (Pr }>\text { ChiSq) }\end{array}$ \\
\hline YearLevel & 10 & 52.52 & $<.0001$ \\
SchoolStatus & 2 & 4.99 & 0.0826 \\
\hline
\end{tabular}


A. SS.4: Estimates and Odds Ratio for Year Level and School Status in 1998

\begin{tabular}{|c|c|c|c|c|c|}
\hline \multicolumn{5}{|c|}{ Analysis Of GEE Parameter Estimates } & \multirow[b]{3}{*}{$\begin{array}{l}\text { Odds } \\
\text { Ratio }\end{array}$} \\
\hline \multicolumn{5}{|c|}{ Empirical Standard Error Estimates } & \\
\hline Parameter & Level & Estimate & $\begin{array}{l}\text { Standard } \\
\text { Error }\end{array}$ & $\begin{array}{l}\text { P-Value } \\
(\mathrm{Pr}>|\mathrm{Z}|)\end{array}$ & \\
\hline Intercept & & -3.8023 & 0.7502 & $<.0001$ & \\
\hline YearLevel & Yr10 & 1.4534 & 0.4038 & 0.0003 & 4.2776 \\
\hline YearLevel & Yr11 & 0.6258 & 0.5791 & 0.2798 & 1.8697 \\
\hline YearLevel & Yr12 & 2.3938 & 0.609 & $<.0001$ & 10.9550 \\
\hline YearLevel & Yr13 & 1.952 & 1.0548 & 0.0642 & 7.0428 \\
\hline YearLevel & Yr2 & 1.5234 & 0.2937 & $<.0001$ & 4.5878 \\
\hline YearLevel & Yr3 & 0.719 & 0.3105 & 0.0206 & 2.0524 \\
\hline YearLevel & Yr4 & 0.5605 & 0.2783 & 0.044 & 1.7515 \\
\hline YearLevel & Yr5 & 0.9426 & 0.3022 & 0.0018 & 2.5666 \\
\hline YearLevel & Yr6 & 0.6435 & 0.2962 & 0.0298 & 1.9031 \\
\hline YearLevel & Yr7 & 0.7979 & 0.2906 & 0.006 & 2.2209 \\
\hline YearLevel & Yr8 & 0 & 0 & & 1.0000 \\
\hline SchoolStatus & Government & -0.087 & 0.6962 & 0.9006 & 0.9167 \\
\hline SchoolStatus & Mission & -1.3942 & 0.8835 & 0.1145 & 0.2480 \\
\hline SchoolStatus & Private & 0 & 0 & & 1.0000 \\
\hline
\end{tabular}

\begin{tabular}{|l|r|r|r|}
\hline \multicolumn{4}{|c|}{ Score Statistics For Type 3 GEE Analysis } \\
\hline \multicolumn{1}{|c|}{ Source } & DF & $\begin{array}{c}\text { Chi- } \\
\text { Square }\end{array}$ & $\begin{array}{c}\text { P-Value } \\
\text { (Pr > ChiSq) }\end{array}$ \\
\hline YearLevel & \multicolumn{1}{c}{ (10 } & 38.69 & $<.0001$ \\
SchoolStatus & 2 & 5.04 & 0.0805 \\
\hline
\end{tabular}


A. SS.5: Estimates and Odds Ratio for Year Level and School Status in 2001

\begin{tabular}{|c|c|c|c|c|c|}
\hline \multicolumn{5}{|c|}{ Analysis Of GEE Parameter Estimates } & \multirow[b]{3}{*}{$\begin{array}{l}\text { Odds } \\
\text { Ratio }\end{array}$} \\
\hline \multicolumn{5}{|c|}{ Empirical Standard Error Estimates } & \\
\hline Parameter & Level & Estimate & $\begin{array}{l}\text { Standard } \\
\text { Error }\end{array}$ & $\begin{array}{l}\text { P-Value } \\
(\operatorname{Pr}>|Z|)\end{array}$ & \\
\hline Intercept & & -3.287 & 0.6552 & $<.0001$ & \\
\hline YearLevel & Yr10 & 1.0619 & 0.2472 & $<.0001$ & 2.8919 \\
\hline YearLevel & Yr11 & 1.061 & 0.2917 & 0.0003 & 2.8893 \\
\hline YearLevel & Yr12 & 1.302 & 0.341 & 0.0001 & 3.6766 \\
\hline YearLevel & Yr13 & 1.0326 & 0.4883 & 0.0345 & 2.8084 \\
\hline YearLevel & Yr2 & 0.851 & 0.2052 & $<.0001$ & 2.3420 \\
\hline YearLevel & Yr3 & 0.361 & 0.2498 & 0.1484 & 1.4348 \\
\hline YearLevel & Yr4 & 0.2537 & 0.1888 & 0.1791 & 1.2888 \\
\hline YearLevel & Yr5 & 0.2799 & 0.2102 & 0.183 & 1.3230 \\
\hline YearLevel & Yr6 & 0.1363 & 0.2043 & 0.5047 & 1.1460 \\
\hline YearLevel & Yr7 & 0.486 & 0.1841 & 0.0083 & 1.6258 \\
\hline YearLevel & Yr8 & 0 & 0 & & 1.0000 \\
\hline SchoolStatus & Government & -0.0348 & 0.6331 & 0.9562 & 0.9658 \\
\hline SchoolStatus & Mission & -0.3701 & 0.658 & 0.5738 & 0.6907 \\
\hline SchoolStatus & Private & 0 & 0 & & 1.0000 \\
\hline
\end{tabular}

\begin{tabular}{|c|c|c|c|}
\hline \multicolumn{4}{|c|}{ Score Statistics For Type 3 GEE Analysis } \\
\hline Source & DF & $\begin{array}{l}\text { Chi- } \\
\text { Square }\end{array}$ & $\begin{array}{c}\text { P-Value } \\
(\mathrm{Pr}>\mathrm{ChiSq})\end{array}$ \\
\hline YearLevel & 10 & 37.4 & $<.0001$ \\
\hline SchoolStatus & 2 & 2.31 & 0.3152 \\
\hline
\end{tabular}


A. SS.6: Estimates and Odds Ratio for Year Level and School Status in 2002

\begin{tabular}{|c|c|c|c|c|c|}
\hline \multicolumn{5}{|c|}{ Analysis Of GEE Parameter Estimates } & \multirow[b]{3}{*}{$\begin{array}{l}\text { Odds } \\
\text { Ratio }\end{array}$} \\
\hline \multicolumn{5}{|c|}{ Empirical Standard Error Estimates } & \\
\hline Parameter & Level & Estimate & $\begin{array}{l}\text { Standard } \\
\text { Error }\end{array}$ & $\begin{array}{c}\text { P-Value } \\
(\operatorname{Pr}>|\mathrm{Z}|)\end{array}$ & \\
\hline Intercept & & -3.1694 & 0.7333 & $<.0001$ & \\
\hline YearLevel & Yr10 & 0.8482 & 0.2479 & 0.0006 & 2.3354 \\
\hline YearLevel & Yr11 & 1.107 & 0.3277 & 0.0007 & 3.0253 \\
\hline YearLevel & Yr12 & 0.848 & 0.4253 & 0.0462 & 2.3350 \\
\hline YearLevel & Yr13 & 0.7121 & 0.3988 & 0.0741 & 2.0383 \\
\hline YearLevel & Yr2 & 0.7806 & 0.1953 & $<.0001$ & 2.1828 \\
\hline YearLevel & Yr3 & 0.0619 & 0.2573 & 0.8099 & 1.0639 \\
\hline YearLevel & Yr4 & -0.1467 & 0.1986 & 0.4599 & 0.8636 \\
\hline YearLevel & Yr5 & 0.3001 & 0.1985 & 0.1306 & 1.3500 \\
\hline YearLevel & Yr6 & 0.0181 & 0.1981 & 0.9272 & 1.0183 \\
\hline YearLevel & Yr7 & 0.2018 & 0.1977 & 0.3074 & 1.2236 \\
\hline YearLevel & Yr8 & 0 & 0 & & 1.0000 \\
\hline SchoolStatus & Government & -0.127 & 0.7132 & 0.8587 & 0.8807 \\
\hline SchoolStatus & Mission & -0.4699 & 0.7407 & 0.5258 & 0.6251 \\
\hline SchoolStatus & Private & 0 & 0 & & 1.0000 \\
\hline
\end{tabular}

Score Statistics For Type 3 GEE Analysis

\begin{tabular}{|l|r|rr|}
\hline \multicolumn{1}{|c|}{ Source } & DF & \multicolumn{1}{c|}{$\begin{array}{c}\text { Chi- } \\
\text { Square }\end{array}$} & $\begin{array}{c}\text { P-Value } \\
\text { (Pr }>\text { ChiSq) }\end{array}$ \\
\hline YearLevel & \multicolumn{1}{c}{10} & 41.45 & $<.0001$ \\
SchoolStatus & 2 & 2.56 & 0.2784 \\
\hline
\end{tabular}


A. SS.7: Estimates and Odds Ratio for Year Level and School Status in 2004

\begin{tabular}{|c|c|c|c|c|c|}
\hline \multicolumn{5}{|c|}{ Analysis Of GEE Parameter Estimates } & \multirow[b]{3}{*}{$\begin{array}{l}\text { Odds } \\
\text { Ratio }\end{array}$} \\
\hline \multicolumn{5}{|c|}{ Empirical Standard Error Estimates } & \\
\hline Parameter & Level & Estimate & $\begin{array}{l}\text { Standard } \\
\text { Error }\end{array}$ & $\begin{array}{c}\text { P-Value } \\
(\operatorname{Pr}>|Z|)\end{array}$ & \\
\hline Intercept & & -3.9025 & 0.6935 & $<.0001$ & \\
\hline YearLevel & Yr10 & 0.6655 & 0.248 & 0.0073 & 1.9455 \\
\hline YearLevel & Yr11 & 0.6985 & 0.3264 & 0.0324 & 2.0107 \\
\hline YearLevel & Yr12 & 0.6422 & 0.3863 & 0.0965 & 1.9007 \\
\hline YearLevel & Yr13 & 0.7371 & 0.4006 & 0.0657 & 2.0899 \\
\hline YearLevel & Yr2 & 0.5583 & 0.1899 & 0.0033 & 1.7477 \\
\hline YearLevel & Yr3 & -0.0986 & 0.1815 & 0.5868 & 0.9061 \\
\hline YearLevel & Yr4 & -0.3586 & 0.2014 & 0.075 & 0.6987 \\
\hline YearLevel & Yr5 & 0.0083 & 0.1839 & 0.9639 & 1.0083 \\
\hline YearLevel & Yr6 & -0.0399 & 0.1823 & 0.8265 & 0.9609 \\
\hline YearLevel & Yr7 & 0.0732 & 0.1882 & 0.6972 & 1.0759 \\
\hline YearLevel & Yr8 & 0 & 0 & & 1.0000 \\
\hline SchoolStatus & Government & 0.8561 & 0.6827 & 0.2099 & 2.3540 \\
\hline SchoolStatus & Mission & 0.7163 & 0.7244 & 0.3228 & 2.0468 \\
\hline SchoolStatus & Private & 0 & 0 & & 1.0000 \\
\hline
\end{tabular}

\begin{tabular}{|l|r|rr|}
\hline \multicolumn{4}{|c|}{ Score Statistics For Type 3 GEE Analysis } \\
\hline Source & DF & $\begin{array}{c}\text { Chi- } \\
\text { Square }\end{array}$ & $\begin{array}{c}\text { P-Value } \\
\text { (Pr > ChiSq) }\end{array}$ \\
\hline YearLevel & 10 & 31.8 & 0.0004 \\
SchoolStatus & 2 & 1.86 & 0.3941 \\
\hline
\end{tabular}


A. SS.8: Estimates and Odds Ratio for Year Level and School Status in 2005

\begin{tabular}{|c|c|c|c|c|c|}
\hline \multicolumn{5}{|c|}{ Analysis Of GEE Parameter Estimates } & \multirow[b]{3}{*}{$\begin{array}{l}\text { Odds } \\
\text { Ratio }\end{array}$} \\
\hline \multicolumn{5}{|c|}{ Empirical Standard Error Estimates } & \\
\hline Parameter & Level & Estimate & $\begin{array}{l}\text { Standard } \\
\text { Error }\end{array}$ & $\begin{array}{l}\text { P-Value } \\
(\operatorname{Pr}>|Z|)\end{array}$ & \\
\hline Intercept & & -2.206 & 0.5365 & $<.0001$ & \\
\hline YearLevel & Yr10 & -0.008 & 0.3253 & 0.9803 & 0.9920 \\
\hline YearLevel & Yr11 & 0.33 & 0.3512 & 0.3475 & 1.3910 \\
\hline YearLevel & Yr12 & -0.0319 & 0.4482 & 0.9432 & 0.9686 \\
\hline YearLevel & Yr13 & 0.8952 & 0.4027 & 0.0262 & 2.4478 \\
\hline YearLevel & Yr2 & 0.5102 & 0.1787 & 0.0043 & 1.6656 \\
\hline YearLevel & Yr3 & -0.0262 & 0.17 & 0.8774 & 0.9741 \\
\hline YearLevel & Yr4 & -0.0005 & 0.1413 & 0.997 & 0.9995 \\
\hline YearLevel & Yr5 & 0.0307 & 0.1256 & 0.8072 & 1.0312 \\
\hline YearLevel & Yr6 & 0.0861 & 0.1439 & 0.5498 & 1.0899 \\
\hline YearLevel & Yr7 & 0.1442 & 0.1379 & 0.2959 & 1.1551 \\
\hline YearLevel & Yr8 & 0 & 0 & & 1.0000 \\
\hline SchoolStatus & Government & -0.7124 & 0.5235 & 0.1736 & 0.4905 \\
\hline SchoolStatus & Mission & -0.4299 & 0.5411 & 0.4269 & 0.6506 \\
\hline SchoolStatus & Private & 0 & 0 & & 1.0000 \\
\hline
\end{tabular}

\begin{tabular}{|l|r|rr|}
\hline \multicolumn{4}{|c|}{ Score Statistics For Type 3 GEE Analysis } \\
\hline \multicolumn{1}{|c|}{ Source } & DF & $\begin{array}{c}\text { Chi- } \\
\text { Square }\end{array}$ & $\begin{array}{c}\text { P-Value } \\
\text { (Pr > ChiSq) }\end{array}$ \\
\hline YearLevel & \multicolumn{1}{c}{10} & 18.66 & 0.0447 \\
SchoolStatus & 2 & 2.4 & 0.3011 \\
\hline
\end{tabular}


A. SS.9: Estimates and Odds Ratio for Year Level and School Status in 2006

\begin{tabular}{|c|c|c|c|c|c|}
\hline \multicolumn{5}{|c|}{ Analysis Of GEE Parameter Estimates } & \multirow[b]{3}{*}{$\begin{array}{l}\text { Odds } \\
\text { Ratio }\end{array}$} \\
\hline \multicolumn{5}{|c|}{ Empirical Standard Error Estimates } & \\
\hline Parameter & Level & Estimate & $\begin{array}{l}\text { Standard } \\
\text { Error }\end{array}$ & $\begin{array}{c}\text { P-Value } \\
(\operatorname{Pr}>|Z|)\end{array}$ & \\
\hline Intercept & & -3.3798 & 0.4303 & $<.0001$ & \\
\hline YearLevel & Yr10 & 0.4343 & 0.2779 & 0.118 & 1.5439 \\
\hline YearLevel & Yr11 & 0.4785 & 0.3034 & 0.1147 & 1.6137 \\
\hline YearLevel & Yr12 & 0.2573 & 0.4356 & 0.5548 & 1.2934 \\
\hline YearLevel & Yr13 & 1.7305 & 0.3407 & $<.0001$ & 5.6435 \\
\hline YearLevel & Yr2 & 0.4869 & 0.1877 & 0.0095 & 1.6273 \\
\hline YearLevel & Yr3 & 0.1076 & 0.1922 & 0.5757 & 1.1136 \\
\hline YearLevel & Yr4 & -0.3442 & 0.2009 & 0.0867 & 0.7088 \\
\hline YearLevel & Yr5 & -0.2806 & 0.2005 & 0.1616 & 0.7553 \\
\hline YearLevel & Yr6 & -0.0151 & 0.1934 & 0.9378 & 0.9850 \\
\hline YearLevel & Yr7 & -0.173 & 0.1702 & 0.3096 & 0.8411 \\
\hline YearLevel & Yr8 & 0 & 0 & & 1.0000 \\
\hline SchoolStatus & Government & 0.5789 & 0.4175 & 0.1656 & 1.7841 \\
\hline SchoolStatus & Mission & 0.1571 & 0.4653 & 0.7357 & 1.1701 \\
\hline SchoolStatus & Private & 0 & 0 & & 1.0000 \\
\hline
\end{tabular}

\begin{tabular}{|c|c|c|c|}
\hline \multicolumn{4}{|c|}{ Score Statistics For Type 3 GEE Analysis } \\
\hline Source & DF & $\begin{array}{l}\text { Chi- } \\
\text { Square }\end{array}$ & $\begin{array}{c}\text { P-Value } \\
(\mathrm{Pr}>\mathrm{ChiSq})\end{array}$ \\
\hline YearLevel & 10 & 48.91 & $<.0001$ \\
\hline SchoolStatus & 2 & 4.33 & 0.1147 \\
\hline
\end{tabular}


A. STR.1: Full Model for the Student-Teacher Ratio Effect in 1995

\begin{tabular}{|c|c|c|c|c|c|}
\hline \multicolumn{5}{|c|}{ Analysis Of GEE Parameter Estimates } & \multirow[b]{3}{*}{$\begin{array}{l}\text { Odds } \\
\text { Ratio }\end{array}$} \\
\hline \multicolumn{5}{|c|}{ Empirical Standard Error Estimates } & \\
\hline Parameter & Level & Estimate & $\begin{array}{c}\text { Standard } \\
\text { Error }\end{array}$ & $\begin{array}{l}\text { P-Value } \\
(\operatorname{Pr}>|Z|)\end{array}$ & \\
\hline Intercept & & -2.2702 & 0.8242 & 0.0059 & \\
\hline StudentTeachRatio & & -0.0379 & 0.0134 & 0.0045 & 0.9628 \\
\hline Region & Apia Urban & -0.1466 & 0.2233 & 0.5115 & 0.8636 \\
\hline Region & Rest of Upolu & 0.2174 & 0.1626 & 0.1812 & 1.2428 \\
\hline Region & Savaii & 0 & 0 & & 1.0000 \\
\hline SchoolStatus & Government & 0.4213 & 0.8079 & 0.602 & 1.5239 \\
\hline SchoolStatus & Mission & 0.3312 & 0.8282 & 0.6892 & 1.3926 \\
\hline SchoolStatus & Private & 0 & 0 & & 1.0000 \\
\hline SchoolLevel & Primary & -0.3539 & 0.2247 & 0.1153 & 0.7019 \\
\hline SchoolLevel & Primary/Secondary & -0.7535 & 0.489 & 0.1233 & 0.4707 \\
\hline SchoolLevel & Secondary & 0 & 0 & & 1.0000 \\
\hline
\end{tabular}

\begin{tabular}{|l|c|c|c|}
\hline \multicolumn{4}{|c|}{ Score Statistics For Type 3 GEE Analysis } \\
\hline \multicolumn{1}{|c}{ Source } & DF & $\begin{array}{c}\text { Chi- } \\
\text { Square }\end{array}$ & $\begin{array}{c}\text { P-Value } \\
\text { (Pr > ChiSq) }\end{array}$ \\
\hline StudentTeachRatio & 1 & 8.45 & 0.0036 \\
Region & 2 & 3.66 & 0.1604 \\
SchoolStatus & 2 & 0.34 & 0.8451 \\
SchoolLevel & 2 & 2.57 & 0.2762 \\
\hline
\end{tabular}


A. STR.2: Full Model for the Student-Teacher Ratio Effect in 1996

\begin{tabular}{|c|c|c|c|c|c|}
\hline \multicolumn{5}{|c|}{ Analysis Of GEE Parameter Estimates } & \multirow[b]{3}{*}{$\begin{array}{l}\text { Odds } \\
\text { Ratio }\end{array}$} \\
\hline \multicolumn{5}{|c|}{ Empirical Standard Error Estimates } & \\
\hline Parameter & Level & Estimate & $\begin{array}{c}\text { Standard } \\
\text { Error }\end{array}$ & $\begin{array}{c}\text { P-Value } \\
(\operatorname{Pr}>|Z|)\end{array}$ & \\
\hline Intercept & & -1.8252 & 0.7579 & 0.016 & \\
\hline StudentTeachRatio & & -0.0205 & 0.01 & 0.0408 & 0.9797 \\
\hline Region & Apia Urban & -0.1213 & 0.2004 & 0.545 & 0.8858 \\
\hline Region & Rest of Upolu & 0.5953 & 0.1362 & $<.0001$ & 1.8136 \\
\hline Region & Savaii & 0 & 0 & & 1.0000 \\
\hline SchoolStatus & Government & -0.0151 & 0.7352 & 0.9836 & 0.9850 \\
\hline SchoolStatus & Mission & -0.3798 & 0.7408 & 0.6082 & 0.6840 \\
\hline SchoolStatus & Private & 0 & 0 & & 1.0000 \\
\hline SchoolLevel & Primary & -0.6042 & 0.1281 & $<.0001$ & 0.5465 \\
\hline SchoolLevel & Primary/Secondary & -0.093 & 0.2043 & 0.649 & 0.9112 \\
\hline SchoolLevel & Secondary & 0 & 0 & & 1.0000 \\
\hline
\end{tabular}

\begin{tabular}{|l|c|r|r|}
\hline \multicolumn{4}{|c|}{ Score Statistics For Type 3 GEE Analysis } \\
\hline \multicolumn{1}{|c}{ Source } & DF & $\begin{array}{c}\text { Chi- } \\
\text { Square }\end{array}$ & $\begin{array}{c}\text { P-Value } \\
\text { (Pr > ChiSq) }\end{array}$ \\
\hline StudentTeachRatio & 1 & 4.64 & 0.0312 \\
Region & 2 & 21.71 & $<.0001$ \\
SchoolStatus & 2 & 3.74 & 0.154 \\
SchoolLevel & 2 & 12.72 & 0.0017 \\
\hline
\end{tabular}


A. STR.3: Full Model for the Student-Teacher Ratio Effect in 1998

\begin{tabular}{|c|c|c|c|c|c|}
\hline \multicolumn{5}{|c|}{ Analysis Of GEE Parameter Estimates } & \multirow[b]{3}{*}{$\begin{array}{l}\text { Odds } \\
\text { Ratio }\end{array}$} \\
\hline \multicolumn{5}{|c|}{ Empirical Standard Error Estimates } & \\
\hline Parameter & Level & Estimate & $\begin{array}{c}\text { Standard } \\
\text { Error }\end{array}$ & $\begin{array}{l}\text { P-Value } \\
(\operatorname{Pr}>|Z|)\end{array}$ & \\
\hline Intercept & & -1.4383 & 0.8082 & 0.0751 & \\
\hline StudentTeachRatio & & -0.0508 & 0.0138 & 0.0002 & 0.9505 \\
\hline Region & Apia Urban & 0.0206 & 0.2327 & 0.9296 & 1.0208 \\
\hline Region & Rest of Upolu & 0.2552 & 0.1449 & 0.0781 & 1.2907 \\
\hline Region & Savaii & 0 & 0 & & 1.0000 \\
\hline SchoolStatus & Government & 0.1338 & 0.83 & 0.8719 & 1.1432 \\
\hline SchoolStatus & Mission & -0.4373 & 0.8759 & 0.6176 & 0.6458 \\
\hline SchoolStatus & Private & 0 & 0 & & 1.0000 \\
\hline SchoolLevel & Primary & -0.4064 & 0.2302 & 0.0775 & 0.6660 \\
\hline SchoolLevel & Primary/Secondary & -0.1016 & 0.4367 & 0.8161 & 0.9034 \\
\hline SchoolLevel & Secondary & 0 & 0 & & 1.0000 \\
\hline
\end{tabular}

\begin{tabular}{|l|c|r|r|}
\hline \multicolumn{4}{|c|}{ Score Statistics For Type 3 GEE Analysis } \\
\hline \multicolumn{1}{|c}{ Source } & DF & $\begin{array}{c}\text { Chi- } \\
\text { Square }\end{array}$ & $\begin{array}{c}\text { P-value } \\
\text { (Pr > ChiSq) }\end{array}$ \\
\hline StudentTeachRatio & 1 & 10.88 & 0.001 \\
Region & 2 & 3.85 & 0.1455 \\
SchoolStatus & 2 & 3.92 & 0.1407 \\
SchoolLevel & 2 & 2.67 & 0.263 \\
\hline
\end{tabular}

A. STR.4: Final Model for the Student-Teacher Ratio effect in 1998

\begin{tabular}{|c|c|c|c|c|}
\hline \multicolumn{4}{|c|}{ Analysis Of GEE Parameter Estimates } & \\
\hline \multicolumn{5}{|c|}{ Empirical Standard Error Estimates } \\
\hline Parameter & Estimate & $\begin{array}{l}\text { Standard } \\
\text { Error }\end{array}$ & $\begin{array}{c}\text { P-Value } \\
(\operatorname{Pr}>|Z|)\end{array}$ & $\begin{array}{l}\text { Odds } \\
\text { Ratio }\end{array}$ \\
\hline Intercept & -1.4731 & 0.2282 & $<.0001$ & \\
\hline StudentTeachRatio & -0.0545 & 0.0104 & $<.0001$ & 0.9470 \\
\hline
\end{tabular}

\begin{tabular}{|c|c|c|c|}
\hline \multicolumn{4}{|c|}{ Score Statistics For Type 3 GEE Analysis } \\
\hline Source & DF & $\begin{array}{c}\text { Chi- } \\
\text { Square }\end{array}$ & $\begin{array}{c}\text { P-Value } \\
\text { (Pr > ChiSq) }\end{array}$ \\
\hline StudentTeachRatio & 1 & 14.72 & 0.0001 \\
\hline
\end{tabular}


A. STR.5: Full Model for the Student-Teacher Ratio Effect in 1999

\begin{tabular}{|c|c|c|c|c|c|}
\hline \multicolumn{5}{|c|}{ Analysis Of GEE Parameter Estimates } & \multirow[b]{3}{*}{$\begin{array}{l}\text { Odds } \\
\text { Ratio }\end{array}$} \\
\hline \multicolumn{5}{|c|}{ Empirical Standard Error Estimates } & \\
\hline Parameter & Level & Estimate & $\begin{array}{c}\text { Standard } \\
\text { Error }\end{array}$ & $\begin{array}{l}\text { P-Value } \\
(\operatorname{Pr}>|Z|)\end{array}$ & \\
\hline Intercept & & -1.6423 & 0.8376 & 0.0499 & \\
\hline StudentTeachRatio & & -0.0569 & 0.0135 & $<.0001$ & 0.9447 \\
\hline Region & Apia Urban & 0.0668 & 0.2882 & 0.8168 & 1.0691 \\
\hline Region & Rest of Upolu & 0.3474 & 0.1697 & 0.0407 & 1.4154 \\
\hline Region & Savaii & 0 & 0 & & 1.0000 \\
\hline SchoolStatus & Government & 0.5277 & 0.8185 & 0.5191 & 1.6950 \\
\hline SchoolStatus & Mission & -0.4363 & 0.8321 & 0.6 & 0.6464 \\
\hline SchoolStatus & Private & 0 & 0 & & 1.0000 \\
\hline SchoolLevel & Primary & -0.5 & 0.2072 & 0.0158 & 0.6065 \\
\hline SchoolLevel & Primary/Secondary & -0.7216 & 0.7631 & 0.3443 & 0.4860 \\
\hline SchoolLevel & Secondary & 0 & 0 & & 1.0000 \\
\hline
\end{tabular}

\begin{tabular}{|l|c|r|r|}
\hline \multicolumn{4}{|c|}{ Score Statistics For Type 3 GEE Analysis } \\
\hline \multicolumn{1}{|c}{ Source } & DF & \multicolumn{1}{c|}{$\begin{array}{c}\text { Chi- } \\
\text { Square }\end{array}$} & $\begin{array}{c}\text { P-Value } \\
\text { (Pr > ChiSq) }\end{array}$ \\
\hline StudentTeachRatio & 1 & 13.67 & 0.0002 \\
Region & 2 & 5.06 & 0.0796 \\
SchoolStatus & 2 & 9.01 & 0.011 \\
SchoolLevel & 2 & 5.12 & 0.0773 \\
\hline
\end{tabular}


A. STR.6: Full Model for the Student-Teacher Ratio Effect in 2000

\begin{tabular}{|c|c|c|c|c|c|}
\hline \multicolumn{5}{|c|}{ Analysis Of GEE Parameter Estimates } & \multirow[b]{3}{*}{$\begin{array}{l}\text { Odds } \\
\text { Ratio }\end{array}$} \\
\hline \multicolumn{5}{|c|}{ Empirical Standard Error Estimates } & \\
\hline Parameter & Level & Estimate & $\begin{array}{l}\text { Standard } \\
\text { Error }\end{array}$ & $\begin{array}{l}\text { P-Value } \\
(\operatorname{Pr}>|Z|)\end{array}$ & \\
\hline Intercept & & -2.2619 & 0.3612 & $<.0001$ & \\
\hline StudentTeachRatio & & -0.0371 & 0.0101 & 0.0003 & 0.9636 \\
\hline Region & Apia Urban & 0.3812 & 0.2264 & 0.0922 & 1.4640 \\
\hline Region & Rest of Upolu & 0.4683 & 0.1521 & 0.0021 & 1.5973 \\
\hline Region & Savaii & 0 & 0 & & 1.0000 \\
\hline SchoolStatus & Government & 0.6857 & 0.3372 & 0.042 & 1.9852 \\
\hline SchoolStatus & Mission & 0.2204 & 0.3436 & 0.5212 & 1.2466 \\
\hline SchoolStatus & Private & 0 & 0 & & 1.0000 \\
\hline SchoolLevel & Primary & -0.394 & 0.187 & 0.0351 & 0.6744 \\
\hline SchoolLevel & Primary/Secondary & -0.6197 & 0.3624 & 0.0873 & 0.5381 \\
\hline SchoolLevel & Secondary & 0 & 0 & & 1.0000 \\
\hline
\end{tabular}

\begin{tabular}{|l|rrrr|}
\hline \multicolumn{4}{|c|}{ Score Statistics For Type 3 GEE Analysis } \\
\hline \multicolumn{1}{|c}{ Source } & DF & $\begin{array}{c}\text { Chi- } \\
\text { Square }\end{array}$ & $\begin{array}{c}\text { P-Value } \\
\text { (Pr > ChiSq) }\end{array}$ \\
\hline StudentTeachRatio & 1 & 9 & 0.0027 \\
Region & 2 & 7.83 & 0.02 \\
SchoolStatus & 2 & 4.71 & 0.0949 \\
SchoolLevel & 2 & 4.53 & 0.1038 \\
\hline
\end{tabular}


A. STR.7: Full Model for the Student-Teacher Ratio Effect in 2001

\begin{tabular}{|c|c|c|c|c|c|}
\hline \multicolumn{5}{|c|}{ Analysis Of GEE Parameter Estimates } & \multirow[b]{3}{*}{$\begin{array}{l}\text { Odds } \\
\text { Ratio }\end{array}$} \\
\hline \multicolumn{5}{|c|}{ Empirical Standard Error Estimates } & \\
\hline Parameter & Level & Estimate & $\begin{array}{c}\text { Standard } \\
\text { Error }\end{array}$ & $\begin{array}{c}\text { P-Value } \\
(\operatorname{Pr}>|Z|)\end{array}$ & \\
\hline Intercept & & -1.8232 & 0.6533 & 0.0053 & \\
\hline StudentTeachRatio & & -0.0284 & 0.0101 & 0.0049 & 0.9720 \\
\hline Region & Apia Urban & 0.0578 & 0.1991 & 0.7717 & 1.0595 \\
\hline Region & Rest of Upolu & 0.383 & 0.1486 & 0.0099 & 1.4667 \\
\hline Region & Savaii & 0 & 0 & & 1.0000 \\
\hline SchoolStatus & Government & 0.0538 & 0.6144 & 0.9302 & 1.0553 \\
\hline SchoolStatus & Mission & -0.296 & 0.6149 & 0.6302 & 0.7438 \\
\hline SchoolStatus & Private & 0 & 0 & & 1.0000 \\
\hline SchoolLevel & Primary & -0.5184 & 0.1829 & 0.0046 & 0.5955 \\
\hline SchoolLevel & Primary/Secondary & 0.132 & 0.3639 & 0.7168 & 1.1411 \\
\hline SchoolLevel & Secondary & 0 & 0 & & 1.0000 \\
\hline
\end{tabular}

\begin{tabular}{|l|c|c|r|}
\hline \multicolumn{4}{|c|}{ Score Statistics For Type 3 GEE Analysis } \\
\hline \multicolumn{1}{|c}{ Source } & DF & $\begin{array}{c}\text { Chi- } \\
\text { Square }\end{array}$ & $\begin{array}{c}\text { P-Value } \\
\text { (Pr > ChiSq) }\end{array}$ \\
\hline StudentTeachRatio & 1 & 9.21 & 0.0024 \\
Region & 2 & 7.91 & 0.0192 \\
SchoolStatus & 2 & 2.94 & 0.2295 \\
SchoolLevel & 2 & 5.32 & 0.07 \\
\hline
\end{tabular}


A. STR.8: Full Model for the Student-Teacher Ratio Effect in 2002

\begin{tabular}{|c|c|c|c|c|c|}
\hline \multicolumn{5}{|c|}{ Analysis Of GEE Parameter Estimates } & \multirow[b]{3}{*}{$\begin{array}{l}\text { Odds } \\
\text { Ratio } \\
\end{array}$} \\
\hline \multicolumn{5}{|c|}{ Empirical Standard Error Estimates } & \\
\hline Parameter & Level & Estimate & $\begin{array}{c}\text { Standard } \\
\text { Error }\end{array}$ & $\begin{array}{l}\text { P-Value } \\
(\operatorname{Pr}>|Z|)\end{array}$ & \\
\hline Intercept & & -1.6824 & 0.7604 & 0.0269 & \\
\hline StudentTeachRatio & & -0.0256 & 0.0126 & 0.043 & 0.9747 \\
\hline Region & Apia Urban & 0.2297 & 0.2093 & 0.2722 & 1.2582 \\
\hline Region & Rest of Upolu & 0.4373 & 0.1586 & 0.0058 & 1.5485 \\
\hline Region & Savaii & 0 & 0 & & 1.0000 \\
\hline SchoolStatus & Government & -0.2824 & 0.7007 & 0.6869 & 0.7540 \\
\hline SchoolStatus & Mission & -0.6045 & 0.7046 & 0.3909 & 0.5463 \\
\hline SchoolStatus & Private & 0 & 0 & & 1.0000 \\
\hline SchoolLevel & Primary & -0.5917 & 0.1765 & 0.0008 & 0.5534 \\
\hline SchoolLevel & Primary/Secondary & -1.4096 & 0.6351 & 0.0264 & 0.2442 \\
\hline SchoolLevel & Secondary & 0 & 0 & & 1.0000 \\
\hline
\end{tabular}

\begin{tabular}{|c|c|c|c|c|}
\hline \multicolumn{5}{|c|}{ Score Statistics For Type 3 GEE Analysis } \\
\hline Source & DF & & $\begin{array}{l}\text { Chi- } \\
\text { Square }\end{array}$ & $\begin{array}{c}\mathrm{P}-\text { Value } \\
(\mathrm{Pr}>\mathrm{ChiSq})\end{array}$ \\
\hline StudentTeachRatio & & 1 & 3.95 & 0.047 \\
\hline Region & & 2 & 6.54 & 0.0381 \\
\hline SchoolStatus & & 2 & 2.57 & 0.2769 \\
\hline SchoolLevel & & 2 & 8.74 & 0.0126 \\
\hline
\end{tabular}

A. STR.9: Final Model for the Student-Teacher Ratio effect in $\mathbf{2 0 0 2}$

\begin{tabular}{|c|c|c|c|c|}
\hline \multicolumn{4}{|c|}{ Analysis Of GEE Parameter Estimates } & \\
\hline \multicolumn{5}{|c|}{ Empirical Standard Error Estimates } \\
\hline Parameter & Estimate & $\begin{array}{l}\text { Standard } \\
\text { Error }\end{array}$ & $\begin{array}{l}\text { P-Value } \\
(\operatorname{Pr}>|Z|)\end{array}$ & $\begin{array}{l}\text { Odds } \\
\text { Ratio }\end{array}$ \\
\hline Intercept & -2.0298 & 0.3151 & $<.0001$ & \\
\hline StudentTeachRatio & -0.0315 & 0.0123 & 0.0106 & 0.9690 \\
\hline
\end{tabular}

\begin{tabular}{|c|c|c|c|}
\hline \multicolumn{4}{|c|}{ Score Statistics For Type 3 GEE Analysis } \\
\hline Source & DF & $\begin{array}{c}\text { Chi- } \\
\text { Square }\end{array}$ & $\begin{array}{c}\text { P-Value } \\
\text { (Pr > ChiSq) }\end{array}$ \\
\hline StudentTeachRatio & 1 & 5.98 & 0.0145 \\
\hline
\end{tabular}


A. STR.10: Full Model for the Student-Teacher Ratio Effect in 2003

\begin{tabular}{|c|c|c|c|c|c|}
\hline \multicolumn{5}{|c|}{ Analysis Of GEE Parameter Estimates } & \multirow[b]{3}{*}{$\begin{array}{l}\text { Odds } \\
\text { Ratio }\end{array}$} \\
\hline \multicolumn{5}{|c|}{ Empirical Standard Error Estimates } & \\
\hline Parameter & Level & Estimate & $\begin{array}{c}\text { Standard } \\
\text { Error }\end{array}$ & $\begin{array}{l}\text { P-Value } \\
(\operatorname{Pr}>|Z|)\end{array}$ & \\
\hline Intercept & & -2.0256 & 0.7004 & 0.0038 & \\
\hline StudentTeachRatio & & -0.0373 & 0.0135 & 0.0058 & 0.9634 \\
\hline Region & Apia Urban & -0.0991 & 0.1962 & 0.6136 & 0.9057 \\
\hline Region & Rest of Upolu & 0.3026 & 0.1535 & 0.0487 & 1.3534 \\
\hline Region & Savaii & 0 & 0 & & 1.0000 \\
\hline SchoolStatus & Government & 0.371 & 0.6809 & 0.5858 & 1.4492 \\
\hline SchoolStatus & Mission & -0.1824 & 0.6912 & 0.7919 & 0.8333 \\
\hline SchoolStatus & Private & 0 & 0 & & 1.0000 \\
\hline SchoolLevel & Primary & -0.4647 & 0.2004 & 0.0204 & 0.6283 \\
\hline SchoolLevel & Primary/Secondary & -1.3354 & 0.6614 & 0.0435 & 0.2631 \\
\hline SchoolLevel & Secondary & 0 & 0 & & 1.0000 \\
\hline
\end{tabular}

\begin{tabular}{|l|c|c|r|}
\hline \multicolumn{4}{|c|}{ Score Statistics For Type 3 GEE Analysis } \\
\hline \multicolumn{1}{|c}{ Source } & DF & $\begin{array}{c}\text { Chi- } \\
\text { Square }\end{array}$ & $\begin{array}{c}\text { P-Value } \\
\text { (Pr > ChiSq) }\end{array}$ \\
\hline StudentTeachRatio & 1 & 8.09 & 0.0045 \\
Region & 2 & 6.39 & 0.0409 \\
SchoolStatus & 2 & 5.75 & 0.0564 \\
SchoolLevel & 2 & 6.34 & 0.0419 \\
\hline
\end{tabular}

A. STR.11: Final Model for the Student-Teacher Ratio effect in 2003

\begin{tabular}{|c|c|c|c|c|}
\hline \multicolumn{4}{|c|}{ Analysis Of GEE Parameter Estimates } & \\
\hline \multicolumn{5}{|c|}{ Empirical Standard Error Estimates } \\
\hline Parameter & Estimate & $\begin{array}{l}\text { Standard } \\
\text { Error }\end{array}$ & $\begin{array}{l}\text { P-Value } \\
(\operatorname{Pr}>|Z|)\end{array}$ & $\begin{array}{l}\text { Odds } \\
\text { Ratio }\end{array}$ \\
\hline Intercept & -1.9117 & 0.2614 & $<.0001$ & \\
\hline StudentTeachRatio & -0.0413 & 0.0099 & $<.0001$ & 0.9595 \\
\hline
\end{tabular}

\begin{tabular}{|c|c|r|r|}
\hline \multicolumn{4}{|c|}{ Score Statistics For Type 3 GEE Analysis } \\
\hline Source & DF & $\begin{array}{c}\text { Chi- } \\
\text { Square }\end{array}$ & $\begin{array}{c}\text { P-Value } \\
\text { (Pr > ChiSq) }\end{array}$ \\
\hline StudentTeachRatio & 1 & 14.59 & 0.0001 \\
\hline
\end{tabular}


A. STR.12: Full Model for the Student-Teacher Ratio Effect in 2004

\begin{tabular}{|c|c|c|c|c|c|}
\hline \multicolumn{5}{|c|}{ Analysis Of GEE Parameter Estimates } & \multirow[b]{3}{*}{$\begin{array}{l}\text { Odds } \\
\text { Ratio }\end{array}$} \\
\hline \multicolumn{5}{|c|}{ Empirical Standard Error Estimates } & \\
\hline Parameter & Level & Estimate & $\begin{array}{l}\text { Standard } \\
\text { Error }\end{array}$ & $\begin{array}{l}\text { P-Value } \\
(\operatorname{Pr}>|\mathrm{Z}|)\end{array}$ & \\
\hline Intercept & & -2.4938 & 0.6274 & $<.0001$ & \\
\hline StudentTeachRatio & & -0.0391 & 0.012 & 0.0011 & 0.9617 \\
\hline Region & Apia Urban & 0.1457 & 0.2327 & 0.5312 & 1.1568 \\
\hline Region & Rest of Upolu & 0.4706 & 0.1692 & 0.0054 & 1.6010 \\
\hline Region & Savaii & 0 & 0 & & 1.0000 \\
\hline SchoolStatus & Government & 0.7679 & 0.5763 & 0.1827 & 2.1552 \\
\hline SchoolStatus & Mission & 0.7291 & 0.6253 & 0.2436 & 2.0732 \\
\hline SchoolStatus & Private & 0 & 0 & & 1.0000 \\
\hline SchoolLevel & Primary & -0.2728 & 0.1736 & 0.1161 & 0.7612 \\
\hline SchoolLevel & Primary/Secondary & -2.1227 & 0.6856 & 0.002 & 0.1197 \\
\hline SchoolLevel & Secondary & 0 & 0 & & 1.0000 \\
\hline
\end{tabular}

\begin{tabular}{|l|c|r|r|}
\hline \multicolumn{4}{|c|}{ Score Statistics For Type 3 GEE Analysis } \\
\hline \multicolumn{1}{|c}{ Source } & DF & $\begin{array}{c}\text { Chi- } \\
\text { Square }\end{array}$ & $\begin{array}{c}\text { P-Value } \\
\text { (Pr > ChiSq) }\end{array}$ \\
\hline StudentTeachRatio & 1 & 9.11 & 0.0025 \\
Region & 2 & 7.86 & 0.0197 \\
SchoolStatus & 2 & 1.5 & 0.4728 \\
SchoolLevel & 2 & 4.96 & 0.0837 \\
\hline
\end{tabular}


A. STR.13: Full Model for the Student-Teacher Ratio Effect in 2005

\begin{tabular}{|c|c|c|c|c|c|}
\hline \multicolumn{5}{|c|}{ Analysis Of GEE Parameter Estimates } & \multirow[b]{3}{*}{$\begin{array}{l}\text { Odds } \\
\text { Ratio }\end{array}$} \\
\hline \multicolumn{5}{|c|}{ Empirical Standard Error Estimates } & \\
\hline Parameter & Level & Estimate & $\begin{array}{l}\text { Standard } \\
\text { Error }\end{array}$ & $\begin{array}{l}\text { P-Value } \\
(\mathrm{Pr}>|\mathrm{Z}|)\end{array}$ & \\
\hline Intercept & & -1.7859 & 0.6355 & 0.005 & \\
\hline StudentTeachRatio & & -0.0432 & 0.0141 & 0.0023 & 0.9577 \\
\hline Region & Apia Urban & 0.5011 & 0.2677 & 0.0612 & 1.6505 \\
\hline Region & Rest of Upolu & 0.4651 & 0.1699 & 0.0062 & 1.5922 \\
\hline Region & Savaii & 0 & 0 & & 1.0000 \\
\hline SchoolStatus & Government & -0.1653 & 0.6642 & 0.8035 & 0.8476 \\
\hline SchoolStatus & Mission & -0.1065 & 0.6381 & 0.8674 & 0.8990 \\
\hline SchoolStatus & Private & 0 & 0 & & 1.0000 \\
\hline SchoolLevel & Primary & 0.2259 & 0.2674 & 0.3983 & 1.2535 \\
\hline SchoolLevel & Primary/Secondary & -0.357 & 0.4452 & 0.4226 & 0.6998 \\
\hline SchoolLevel & Secondary & 0 & 0 & & 1.0000 \\
\hline
\end{tabular}

\begin{tabular}{|l|c|r|r|}
\hline \multicolumn{4}{|c|}{ Score Statistics For Type 3 GEE Analysis } \\
\hline \multicolumn{1}{|c}{ Source } & DF & $\begin{array}{c}\text { Chi- } \\
\text { Square }\end{array}$ & $\begin{array}{c}\text { P-Value } \\
\text { (Pr > ChiSq) }\end{array}$ \\
\hline StudentTeachRatio & 1 & 6.16 & 0.0131 \\
Region & 2 & 6.7 & 0.035 \\
SchoolStatus & 2 & 0.1 & 0.9511 \\
SchoolLevel & 2 & 1.38 & 0.5015 \\
\hline
\end{tabular}


A. STR.14: Full Model for the Student-Teacher Ratio Effect in 2006

\begin{tabular}{|c|c|c|c|c|c|}
\hline \multicolumn{5}{|c|}{ Analysis Of GEE Parameter Estimates } & \multirow[b]{3}{*}{$\begin{array}{l}\text { Odds } \\
\text { Ratio }\end{array}$} \\
\hline \multicolumn{5}{|c|}{ Empirical Standard Error Estimates } & \\
\hline Parameter & Level & Estimate & $\begin{array}{c}\text { Standard } \\
\text { Error }\end{array}$ & $\begin{array}{c}\text { P-Value } \\
(\operatorname{Pr}>|Z|)\end{array}$ & \\
\hline Intercept & & -1.9354 & 0.3076 & $<.0001$ & \\
\hline StudentTeachRatio & & -0.0373 & 0.0112 & 0.0009 & 0.9634 \\
\hline Region & Apia Urban & 0.1923 & 0.1496 & 0.1986 & 1.2120 \\
\hline Region & Rest of Upolu & 0.4519 & 0.1252 & 0.0003 & 1.5713 \\
\hline Region & Savaii & 0 & 0 & & 1.0000 \\
\hline SchoolStatus & Government & 0.4179 & 0.249 & 0.0933 & 1.5188 \\
\hline SchoolStatus & Mission & -0.1104 & 0.3043 & 0.7168 & 0.8955 \\
\hline SchoolStatus & Private & 0 & 0 & & 1.0000 \\
\hline SchoolLevel & Primary & -0.2257 & 0.1554 & 0.1465 & 0.7980 \\
\hline SchoolLevel & Primary/Secondary & -0.5606 & 0.3865 & 0.1469 & 0.5709 \\
\hline SchoolLevel & Secondary & 0 & 0 & & 1.0000 \\
\hline
\end{tabular}

\begin{tabular}{|l|c|r|r|}
\hline \multicolumn{4}{|c|}{ Score Statistics For Type 3 GEE Analysis } \\
\hline \multicolumn{1}{|c}{ Source } & DF & $\begin{array}{c}\text { Chi- } \\
\text { Square }\end{array}$ & $\begin{array}{c}\text { P-Value } \\
\text { (Pr > ChiSq) }\end{array}$ \\
\hline StudentTeachRatio & 1 & 24.6 & $<.0001$ \\
Region & 2 & 11.47 & 0.0032 \\
SchoolStatus & 2 & 7.36 & 0.0253 \\
SchoolLevel & 2 & 3.37 & 0.1859 \\
\hline
\end{tabular}


A. SS-TE.1: The Model for the School Size (Total Enrolment) Effect in 1996

\begin{tabular}{|ccccc|}
\hline \multicolumn{4}{|c|}{ Analysis Of GEE Parameter Estimates } & \multirow{2}{*}{} \\
\cline { 1 - 3 } Empirical Standard Error Estimates & \\
\cline { 1 - 3 } Parameter & Estimate & $\begin{array}{c}\text { Standard } \\
\text { Error }\end{array}$ & $\begin{array}{c}\text { P-Value } \\
\text { (Pr > |Z|) }\end{array}$ & $\begin{array}{c}\text { Odd } \\
\text { Ratio }\end{array}$ \\
\hline Intercept & -2.3161 & 0.131 & $<.0001$ & \\
TotEnrol & -0.0008 & 0.0004 & 0.0656 & 0.9992 \\
\hline
\end{tabular}

Score Statistics For Type 3 GEE Analysis

\begin{tabular}{|l|r|r|r|}
\hline Source & DF & $\begin{array}{c}\text { Chi- } \\
\text { Square }\end{array}$ & $\begin{array}{c}\text { P-Value } \\
(\mathbf{P r}>\text { ChiSq })\end{array}$ \\
\hline TotEnrol & \multicolumn{1}{|c}{1} & 9.03 & 0.0027 \\
\hline
\end{tabular}

A. SS-TE.2: The Model for the School Size (Total Enrolment) Effect in 1997

\begin{tabular}{|ccc|c|c|c|}
\hline \multicolumn{4}{|c|}{ Analysis Of GEE Parameter Estimates } & \multirow{2}{*}{} \\
\cline { 1 - 3 } Empirical Standard Error Estimates & \multirow{2}{*}{ Parameter } & Estimate & $\begin{array}{c}\text { Standard } \\
\text { Error }\end{array}$ & $\begin{array}{c}\text { P-Value } \\
(\text { Pr }>|Z|)\end{array}$ & $\begin{array}{c}\text { Odd } \\
\text { Ratio }\end{array}$ \\
\hline Intercept & -2.2913 & 0.1412 & $<.0001$ & \\
TotEnrol & -0.001 & 0.0005 & 0.0244 & 0.9990 \\
\hline
\end{tabular}

\begin{tabular}{|c|c|c|c|}
\hline \multicolumn{4}{|c|}{ Score Statistics For Type 3 GEE Analysis } \\
\hline Source & DF & $\begin{array}{c}\text { Chi- } \\
\text { Square }\end{array}$ & $\begin{array}{c}\text { P-Value } \\
\text { (Pr > ChiSq) }\end{array}$ \\
\hline TotEnrol & \multicolumn{1}{|c}{1} & 8.17 & 0.0043 \\
\hline
\end{tabular}


A. SS-TE.3: The Model for the School Size (Total Enrolment) Effect in 1998

\begin{tabular}{|c|c|c|c|c|}
\hline \multicolumn{4}{|c|}{ Analysis Of GEE Parameter Estimates } & \multirow{2}{*}{} \\
\cline { 1 - 3 } Empirical Standard Error Estimates & \\
\hline Parameter & Estimate & $\begin{array}{c}\text { Standard } \\
\text { Error }\end{array}$ & $\begin{array}{c}\text { P-Value } \\
(\text { Pr }>|\mathbf{Z}|)\end{array}$ & $\begin{array}{c}\text { Odd } \\
\text { Ratio }\end{array}$ \\
\hline Intercept & -2.4073 & 0.2035 & $<.0001$ & \\
TotEnrol & -0.0009 & 0.0007 & 0.1871 & 0.9991 \\
\hline
\end{tabular}

\begin{tabular}{|c|c|c|c|}
\hline \multicolumn{4}{|c|}{ Score Statistics For Type 3 GEE Analysis } \\
\hline Source & DF & $\begin{array}{c}\text { Chi- } \\
\text { Square }\end{array}$ & $\begin{array}{c}\text { P-Value } \\
(\mathbf{P r}>\text { ChiSq) }\end{array}$ \\
\hline TotEnrol & 1 & 4.13 & 0.0421 \\
\hline
\end{tabular}

A. SS-TE.4: The Model for the School Size (Total Enrolment) Effect in 1999

\begin{tabular}{|c|c|c|c|c|}
\hline \multicolumn{4}{|c|}{ Analysis Of GEE Parameter Estimates } & \multirow[b]{3}{*}{$\begin{array}{l}\text { Odd } \\
\text { Ratio }\end{array}$} \\
\hline \multicolumn{4}{|c|}{ Empirical Standard Error Estimates } & \\
\hline Parameter & Estimate & $\begin{array}{l}\text { Standard } \\
\text { Error }\end{array}$ & $\begin{array}{c}\text { P-Value } \\
(\mathrm{Pr}>|\mathrm{Z}|)\end{array}$ & \\
\hline Intercept & -2.2353 & 0.2384 & $<.0001$ & \\
\hline TotEnrol & -0.0014 & 0.0009 & 0.0902 & 0.9986 \\
\hline
\end{tabular}

\begin{tabular}{|c|c|c|c|}
\hline \multicolumn{4}{|c|}{ Score Statistics For Type 3 GEE Analysis } \\
\hline Source & DF & $\begin{array}{l}\text { Chi- } \\
\text { Square }\end{array}$ & $\begin{array}{c}\text { P-Value } \\
(\mathrm{Pr}>\mathrm{ChiSq})\end{array}$ \\
\hline TotEnrol & \multicolumn{2}{|c|}{19.84} & 0.0017 \\
\hline
\end{tabular}


A. SS-TE.5: The Model for the School Size (Total Enrolment) Effect in 2000

\begin{tabular}{|c|c|c|c|c|}
\hline \multicolumn{4}{|c|}{ Analysis Of GEE Parameter Estimates } & \\
\hline \multicolumn{4}{|c|}{ Empirical Standard Error Estimates } & \\
\hline Parameter & Estimate & $\begin{array}{c}\text { Standard } \\
\text { Error }\end{array}$ & $\begin{array}{l}\text { P-Value } \\
(\operatorname{Pr}>|Z|)\end{array}$ & $\begin{array}{l}\text { Odd } \\
\text { Ratio }\end{array}$ \\
\hline Intercept & -2.3432 & 0.1597 & $<.0001$ & \\
\hline TotEnrol & -0.0005 & 0.0005 & 0.2399 & 0.9995 \\
\hline
\end{tabular}

\begin{tabular}{|c|c|c|c|}
\hline \multicolumn{4}{|c|}{ Score Statistics For Type 3 GEE Analysis } \\
\hline Source & DF & $\begin{array}{c}\text { Chi- } \\
\text { Square }\end{array}$ & $\begin{array}{c}\text { P-Value } \\
\text { (Pr > ChiSq) }\end{array}$ \\
\hline TotEnrol & 1 & 3.29 & 0.0697 \\
\hline
\end{tabular}

A. SS-TE.6: The Model for the School Size (Total Enrolment) Effect in 2001

\begin{tabular}{|c|c|c|c|c|}
\hline \multicolumn{4}{|c|}{ Analysis Of GEE Parameter Estimates } & \\
\hline \multicolumn{4}{|c|}{ Empirical Standard Error Estimates } & \\
\hline Parameter & Estimate & $\begin{array}{l}\text { Standard } \\
\text { Error }\end{array}$ & $\begin{array}{c}\text { P-Value } \\
(\mathrm{Pr}>|\mathrm{Z}|)\end{array}$ & $\begin{array}{l}\text { Odd } \\
\text { Ratio }\end{array}$ \\
\hline Intercept & -2.4996 & 0.1861 & $<.0001$ & \\
\hline TotEnrol & -0.0006 & 0.0006 & 0.3326 & 0.9994 \\
\hline
\end{tabular}

\begin{tabular}{|c|c|c|c|}
\hline \multicolumn{4}{|c|}{ Score Statistics For Type $\mathbf{3}$ GEE Analysis } \\
\hline Source & DF & $\begin{array}{c}\text { Chi- } \\
\text { Square }\end{array}$ & $\begin{array}{c}\text { P-Value } \\
(\text { Pr }>\text { ChiSq) }\end{array}$ \\
\hline TotEnrol & 1 & 1.65 & 0.1989 \\
\hline
\end{tabular}


A. SS-TE.7: The Model for the School Size (Total Enrolment) Effect in 2002

\begin{tabular}{|c|c|c|c|c|}
\hline \multicolumn{4}{|c|}{ Analysis Of GEE Parameter Estimates } & \\
\hline \multicolumn{5}{|c|}{ Empirical Standard Error Estimates } \\
\hline Parameter & Estimate & $\begin{array}{l}\text { Standard } \\
\text { Error }\end{array}$ & $\begin{array}{l}\text { P-Value } \\
(\operatorname{Pr}>|Z|)\end{array}$ & $\begin{array}{l}\text { Odd } \\
\text { Ratio }\end{array}$ \\
\hline Intercept & -2.8587 & 0.1321 & $<.0001$ & \\
\hline TotEnrol & 0 & 0.0003 & 0.9263 & 1 \\
\hline
\end{tabular}

Score Statistics For Type 3 GEE Analysis

\begin{tabular}{|c|c|c|c|}
\hline Source & DF & $\begin{array}{c}\text { Chi- } \\
\text { Square }\end{array}$ & $\begin{array}{c}\text { P-Value } \\
(\mathbf{P r}>\text { ChiSq })\end{array}$ \\
\hline TotEnrol & 1 & 0.01 & 0.9248 \\
\hline
\end{tabular}

A. SS-TE.8: The Model for the School Size (Total Enrolment) Effect in 2003

\begin{tabular}{|c|c|c|c|c|}
\hline \multicolumn{4}{|c|}{ Analysis Of GEE Parameter Estimates } & \multirow{2}{*}{} \\
\cline { 1 - 3 } Empirical Standard Error Estimates & \\
\hline Parameter & Estimate & $\begin{array}{c}\text { Standard } \\
\text { Error }\end{array}$ & $\begin{array}{c}\text { P-Value } \\
\text { (Pr > |Z|) }\end{array}$ & $\begin{array}{c}\text { Odd } \\
\text { Ratio }\end{array}$ \\
\hline Intercept & -2.7965 & 0.1884 & $<.0001$ & \\
TotEnrol & -0.0005 & 0.0005 & 0.3338 & 0.9995 \\
\hline
\end{tabular}

\begin{tabular}{|c|c|c|c|}
\hline \multicolumn{4}{|c|}{ Score Statistics For Type 3 GEE Analysis } \\
\hline Source & DF & $\begin{array}{c}\text { Chi- } \\
\text { Square }\end{array}$ & $\begin{array}{c}\text { P-Value } \\
\text { (Pr }>\text { ChiSq) }\end{array}$ \\
\hline TotEnrol & 1 & 2.23 & 0.1357 \\
\hline
\end{tabular}


A. SS-TE.9: The Model for the School Size (Total Enrolment) Effect in 2004

\begin{tabular}{|c|c|c|c|c|}
\hline \multicolumn{4}{|c|}{ Analysis Of GEE Parameter Estimates } & \\
\hline \multicolumn{5}{|c|}{ Empirical Standard Error Estimates } \\
\hline Parameter & Estimate & $\begin{array}{l}\text { Standard } \\
\text { Error }\end{array}$ & $\begin{array}{c}\text { P-Value } \\
(\operatorname{Pr}>|Z|)\end{array}$ & $\begin{array}{l}\text { Odd } \\
\text { Ratio }\end{array}$ \\
\hline Intercept & -2.6879 & 0.1656 & $<.0001$ & \\
\hline TotEnrol & -0.0002 & 0.0004 & 0.5768 & 0.9998 \\
\hline
\end{tabular}

\begin{tabular}{|c|c|c|c|}
\hline \multicolumn{4}{|c|}{ Score Statistics For Type 3 GEE Analysis } \\
\hline Source & DF & $\begin{array}{c}\text { Chi- } \\
\text { Square }\end{array}$ & $\begin{array}{c}\text { P-Value } \\
\text { (Pr > ChiSq) }\end{array}$ \\
\hline TotEnrol & 1 & 0.48 & 0.4877 \\
\hline
\end{tabular}

A. SS-TE.10: The Model for the School Size (Total Enrolment) Effect in 2005

\begin{tabular}{|c|c|c|c|c|}
\hline \multicolumn{4}{|c|}{ Analysis Of GEE Parameter Estimates } & \\
\hline \multicolumn{4}{|c|}{ Empirical Standard Error Estimates } & \\
\hline Parameter & Estimate & $\begin{array}{l}\text { Standard } \\
\text { Error }\end{array}$ & $\begin{array}{l}\text { P-Value } \\
(\operatorname{Pr}>|Z|)\end{array}$ & $\begin{array}{l}\text { Odd } \\
\text { Ratio }\end{array}$ \\
\hline Intercept & -2.4837 & 0.1472 & $<.0001$ & \\
\hline TotEnrol & -0.0005 & 0.0003 & 0.1552 & 0.9995 \\
\hline
\end{tabular}

\begin{tabular}{|c|c|c|c|}
\hline \multicolumn{4}{|c|}{ Score Statistics For Type 3 GEE Analysis } \\
\hline Source & DF & $\begin{array}{c}\text { Chi- } \\
\text { Square }\end{array}$ & $\begin{array}{c}\text { P-Value } \\
\text { (Pr }>\text { ChiSq) }\end{array}$ \\
\hline TotEnrol & 1 & 3.75 & 0.0529 \\
\hline
\end{tabular}




\section{B. Secondary Schools' Other Models (2007)}

B. SSTV. 1: The Full Model for 2007 Secondary School Teacher Variables

\begin{tabular}{|c|c|c|c|c|}
\hline \multicolumn{4}{|c|}{ Analysis Of GEE Parameter Estimates } & \\
\hline \multicolumn{5}{|c|}{ Empirical Standard Error Estimates } \\
\hline Parameter & Estimate & $\begin{array}{l}\text { Standard } \\
\text { Error }\end{array}$ & $\begin{array}{c}\text { P-Value } \\
(\operatorname{Pr}>|Z|)\end{array}$ & $\begin{array}{l}\text { Odds } \\
\text { Ratio }\end{array}$ \\
\hline Intercept & 0.6759 & 0.7954 & 0.3955 & \\
\hline PropFemales & 0.3185 & 0.4819 & 0.5087 & 1.3751 \\
\hline PropSamoans & -3.5366 & 0.8358 & $<.0001$ & 0.0291 \\
\hline Certificates & 0.0336 & 0.0054 & $<.0001$ & 1.0342 \\
\hline Degrees & -0.0296 & 0.0086 & 0.0005 & 0.9708 \\
\hline
\end{tabular}

\begin{tabular}{|l|r|r|r|}
\hline \multicolumn{4}{|c|}{ Score Statistics For Type 3 GEE Analysis } \\
\hline \multicolumn{1}{|c|}{ Source } & DF & $\begin{array}{c}\text { Chi- } \\
\text { Square }\end{array}$ & $\begin{array}{c}\text { P-Value } \\
(\text { Pr }>\text { ChiSq })\end{array}$ \\
\hline PropFemales & 1 & 0.45 & 0.5042 \\
PropSamoans & 1 & 4.67 & 0.0308 \\
Certificates & 1 & 3.24 & 0.0717 \\
Degrees & 1 & 6.16 & 0.0131 \\
\hline
\end{tabular}

B. SSTV. 2: The Other Model for 2007 Secondary School Teacher Variables

\begin{tabular}{|lrrr|r|}
\hline \multicolumn{4}{|c|}{ Analysis Of GEE Parameter Estimates } & \multirow{2}{*}{} \\
\cline { 1 - 3 } Empirical Standard Error Estimates & \multirow{2}{*}{ Odds } \\
Parameter & Estimate & $\begin{array}{c}\text { Standard } \\
\text { Error }\end{array}$ & $\begin{array}{c}\text { P-Value } \\
(\mathbf{P r}>|\mathbf{Z}|)\end{array}$ & Ratio \\
\hline Intercept & -0.0444 & 1.0435 & 0.9661 & \\
PropSamoans & -2.0151 & 1.146 & 0.0787 & 0.1333 \\
Degrees & -0.0178 & 0.0086 & 0.0383 & 0.9824 \\
\hline
\end{tabular}

\begin{tabular}{|l|c|r|r|}
\hline \multicolumn{4}{|c|}{ Score Statistics For Type 3 GEE Analysis } \\
\hline Source & DF & $\begin{array}{c}\text { Chi- } \\
\text { Square }\end{array}$ & $\begin{array}{c}\text { P-Value } \\
\text { (Pr > ChiSq) }\end{array}$ \\
\hline PropSamoans & 1 & 1.81 & 0.1783 \\
Degrees & 1 & 2.09 & 0.1482 \\
\hline
\end{tabular}


B. SSB V. 1: The Full Model for 2007 Secondary School Building Variables

\begin{tabular}{|c|c|c|c|c|}
\hline \multicolumn{4}{|c|}{ Analysis Of GEE Parameter Estimates } & \multirow[b]{3}{*}{$\begin{array}{l}\text { Odds } \\
\text { Ratio }\end{array}$} \\
\hline \multicolumn{4}{|c|}{ Empirical Standard Error Estimates } & \\
\hline Parameter & Estimate & $\begin{array}{l}\text { Standard } \\
\text { Error }\end{array}$ & $\begin{array}{c}\text { P-Value } \\
(\operatorname{Pr}>|Z|)\end{array}$ & \\
\hline Intercept & -3.3617 & 0.6679 & $<.0001$ & \\
\hline TotEnrol & 0.0021 & 0.0015 & 0.1479 & 1.0021 \\
\hline Classrooms & -0.0921 & 0.0643 & 0.1519 & 0.9120 \\
\hline ClassroomArea & -0.0003 & 0.0003 & 0.3146 & 0.9997 \\
\hline Offices & -0.3118 & 0.2065 & 0.1309 & 0.7321 \\
\hline OfficeArea & 0.0177 & 0.0058 & 0.0023 & 1.0179 \\
\hline Storerooms & 0.0483 & 0.056 & 0.3875 & 1.0495 \\
\hline StoreroomArea & -0.0202 & 0.006 & 0.0007 & 0.9800 \\
\hline HomeEc & 0.6294 & 0.3233 & 0.0516 & 1.8765 \\
\hline HomeEcArea & 0.0014 & 0.003 & 0.6499 & 1.0014 \\
\hline Artrooms & -0.3965 & 0.5033 & 0.4308 & 0.6727 \\
\hline ArtroomArea & 0.0135 & 0.0042 & 0.0015 & 1.0136 \\
\hline ScienceLab & -0.748 & 0.5072 & 0.1403 & 0.4733 \\
\hline ScienceLabArea & 0.0277 & 0.005 & $<.0001$ & 1.0281 \\
\hline Staffrooms & 1.2609 & 0.6012 & 0.036 & 3.5286 \\
\hline StaffroomArea & -0.0208 & 0.0087 & 0.0169 & 0.9794 \\
\hline Libraries & -0.8968 & 0.6081 & 0.1403 & 0.4079 \\
\hline LibraryArea & -0.0022 & 0.003 & 0.4574 & 0.9978 \\
\hline Fales & 0.2704 & 0.168 & 0.1076 & 1.3105 \\
\hline FaleArea & 0.0023 & 0.001 & 0.0215 & 1.0023 \\
\hline Halls & 0.1716 & 0.3773 & 0.6492 & 1.1872 \\
\hline HallArea & -0.0002 & 0.0006 & 0.7592 & 0.9998 \\
\hline Workshops & 0.1283 & 0.3446 & 0.7096 & 1.1369 \\
\hline WorkShopArea & 0.0004 & 0.001 & 0.7051 & 1.0004 \\
\hline Canteens & 0.0291 & 0.4103 & 0.9435 & 1.0295 \\
\hline CanteenArea & 0.0043 & 0.0037 & 0.249 & 1.0043 \\
\hline ComputerLabs & -0.1527 & 0.3085 & 0.6206 & 0.8584 \\
\hline ComputerLabArea & -0.0271 & 0.005 & $<.0001$ & 0.9733 \\
\hline Toilets & 0.0401 & 0.0276 & 0.146 & 1.0409 \\
\hline Urinals & -0.0471 & 0.1128 & 0.6764 & 0.9540 \\
\hline Washbasins & 0.0426 & 0.0428 & 0.32 & 1.0435 \\
\hline Drinkfountains & 0.65 & 0.1698 & 0.0001 & 1.9155 \\
\hline
\end{tabular}




\begin{tabular}{|l|r|r|r|}
\hline \multicolumn{4}{|c|}{ Score Statistics For Type 3 GEE Analysis } \\
\hline \multicolumn{1}{|c|}{ Source } & DF & $\begin{array}{c}\text { Chi- } \\
\text { Square }\end{array}$ & $\begin{array}{c}\text { P-Value } \\
\text { (Pr > ChiSq) }\end{array}$ \\
\hline TotEnrol & 1 & 3.85 & 0.0498 \\
Classrooms & 1 & 2.68 & 0.1014 \\
ClassroomArea & 1 & 1.29 & 0.2559 \\
Offices & 1 & 1.78 & 0.1823 \\
OfficeArea & 1 & 5.61 & 0.0179 \\
Storerooms & 1 & 0.64 & 0.4252 \\
StoreroomArea & 1 & 4.89 & 0.0269 \\
HomeEc & 1 & 2.21 & 0.1368 \\
HomeEcArea & 1 & 0.23 & 0.632 \\
Artrooms & 1 & 0.71 & 0.399 \\
ArtroomArea & 1 & 4.13 & 0.0422 \\
ScienceLab & 1 & 2.97 & 0.0847 \\
ScienceLabArea & 1 & 13.13 & 0.0003 \\
Staffrooms & 1 & 2.5 & 0.1142 \\
StaffroomArea & 1 & 4.08 & 0.0433 \\
Libraries & 1 & 1.4 & 0.2367 \\
LibraryArea & 1 & 0.55 & 0.4571 \\
Fales & 1 & 1.97 & 0.1607 \\
FaleArea & 1 & 3.72 & 0.0536 \\
Halls & 1 & 0.22 & 0.6357 \\
HallArea & 1 & 0.12 & 0.7306 \\
Workshops & 1 & 0.14 & 0.7061 \\
WorkShopArea & 1 & 0.16 & 0.6868 \\
Canteens & 1 & 0.01 & 0.9415 \\
CanteenArea & 1 & 1.27 & 0.2605 \\
ComputerLabs & 1 & 0.25 & 0.6178 \\
ComputerLabArea & 1 & 7.84 & 0.0051 \\
Toilets & 1 & 1.73 & 0.1889 \\
Urinals & 1 & 0.15 & 0.6985 \\
Washbasins & 1 & 1.75 & 0.1861 \\
Drinkfountains & 1 & 8.17 & 0.0043 \\
\hline
\end{tabular}


B. SSFV. 1: The Full Model for 2007 Secondary School Facility Variables

\begin{tabular}{|c|c|c|c|c|}
\hline \multicolumn{4}{|c|}{ Analysis Of GEE Parameter Estimates } & \multirow[b]{3}{*}{$\begin{array}{l}\text { Odds } \\
\text { Ratio }\end{array}$} \\
\hline \multicolumn{4}{|c|}{ Empirical Standard Error Estimates } & \\
\hline Parameter & Estimate & $\begin{array}{c}\text { Standard } \\
\text { Error }\end{array}$ & $\begin{array}{l}\text { P-Value } \\
(\operatorname{Pr}>|\mathrm{Z}|)\end{array}$ & \\
\hline Intercept & -2.2723 & 0.5952 & 0.0001 & \\
\hline TotEnrol & -0.0041 & 0.0029 & 0.152 & 0.9959 \\
\hline Desks & 0.0025 & 0.0021 & 0.2321 & 1.0025 \\
\hline Seats & -0.0015 & 0.0007 & 0.0336 & 0.9985 \\
\hline TeacherDesks & -0.0215 & 0.0772 & 0.781 & 0.9787 \\
\hline TeacherChairs & 0.0313 & 0.0893 & 0.7256 & 1.0318 \\
\hline Blackboards & -0.0074 & 0.0146 & 0.6125 & 0.9926 \\
\hline StaffTables & 0.1012 & 0.0587 & 0.0845 & 1.1065 \\
\hline StaffChairs & 0.0372 & 0.0126 & 0.0032 & 1.0379 \\
\hline StaffCupboards & 0.0381 & 0.0156 & 0.0145 & 1.0388 \\
\hline StaffBlackboards & 0.183 & 0.1967 & 0.3523 & 1.2008 \\
\hline StaffPinboards & 0.0483 & 0.0296 & 0.1029 & 1.0495 \\
\hline StaffSinks & -0.3945 & 0.2025 & 0.0515 & 0.6740 \\
\hline Buses & -0.4859 & 0.2751 & 0.0774 & 0.6151 \\
\hline Trucks & -1.4178 & 0.4244 & 0.0008 & 0.2422 \\
\hline Vehicles & -0.2675 & 0.2312 & 0.2471 & 0.7653 \\
\hline Duplicators & -0.4184 & 0.6604 & 0.5264 & 0.6581 \\
\hline Photocopiers & -0.0899 & 0.3448 & 0.7944 & 0.9140 \\
\hline Computers & 0.0247 & 0.0119 & 0.0387 & 1.0250 \\
\hline Radios & -0.1513 & 0.0692 & 0.0287 & 0.8596 \\
\hline TVs & 0.4112 & 0.2458 & 0.0944 & 1.5086 \\
\hline Projectors & 0.029 & 0.1275 & 0.8199 & 1.0294 \\
\hline FireExtinguisher & -0.481 & 0.1449 & 0.0009 & 0.6182 \\
\hline FirstAid & 0.376 & 0.2863 & 0.1891 & 1.4564 \\
\hline Phones & -0.0281 & 0.2607 & 0.9143 & 0.9723 \\
\hline Fax & 0.9105 & 0.2613 & 0.0005 & 2.4856 \\
\hline Internet & -0.3134 & 0.4187 & 0.4541 & 0.7310 \\
\hline
\end{tabular}




\begin{tabular}{|l|r|r|r|}
\hline \multicolumn{4}{|c|}{ Score Statistics For Type 3 GEE Analysis } \\
\hline \multicolumn{1}{|c|}{ Source } & DF & $\begin{array}{c}\text { Chi- } \\
\text { Square }\end{array}$ & $\begin{array}{c}\text { P-Value } \\
\text { (Pr > ChiSq) }\end{array}$ \\
\hline TotEnrol & 1 & 1.55 & 0.2135 \\
Desks & 1 & 1.6 & 0.206 \\
Seats & 1 & 3.5 & 0.0612 \\
TeacherDesks & 1 & 0.07 & 0.7896 \\
TeacherChairs & 1 & 0.11 & 0.741 \\
Blackboards & 1 & 0.25 & 0.6168 \\
StaffTables & 1 & 2.88 & 0.0896 \\
StaffChairs & 1 & 5.79 & 0.0162 \\
StaffCupboards & 1 & 3.37 & 0.0663 \\
StaffBlackboards & 1 & 0.92 & 0.3376 \\
StaffPinboards & 1 & 1.8 & 0.1795 \\
StaffSinks & 1 & 2.7 & 0.1004 \\
Buses & 1 & 2.03 & 0.1545 \\
Trucks & 1 & 6.61 & 0.0102 \\
Vehicles & 1 & 1 & 0.3183 \\
Duplicators & 1 & 0.42 & 0.5179 \\
Photocopiers & 1 & 0.07 & 0.79 \\
Computers & 1 & 3.39 & 0.0654 \\
Radios & 1 & 5.05 & 0.0246 \\
TVs & 1 & 2.71 & 0.0995 \\
Projectors & 1 & 0.06 & 0.8095 \\
FireExtinguisher & 1 & 3.97 & 0.0463 \\
FirstAid & 1 & 2.03 & 0.1545 \\
Phones & 1 & 0.01 & 0.9077 \\
Fax & 1 & 5.98 & 0.0145 \\
Internet & 1 & 0.52 & 0.4692 \\
\hline
\end{tabular}




\section{More Figures}

C. RDR. G1: Comparing Yr 3 Level Dropout Rates by Region

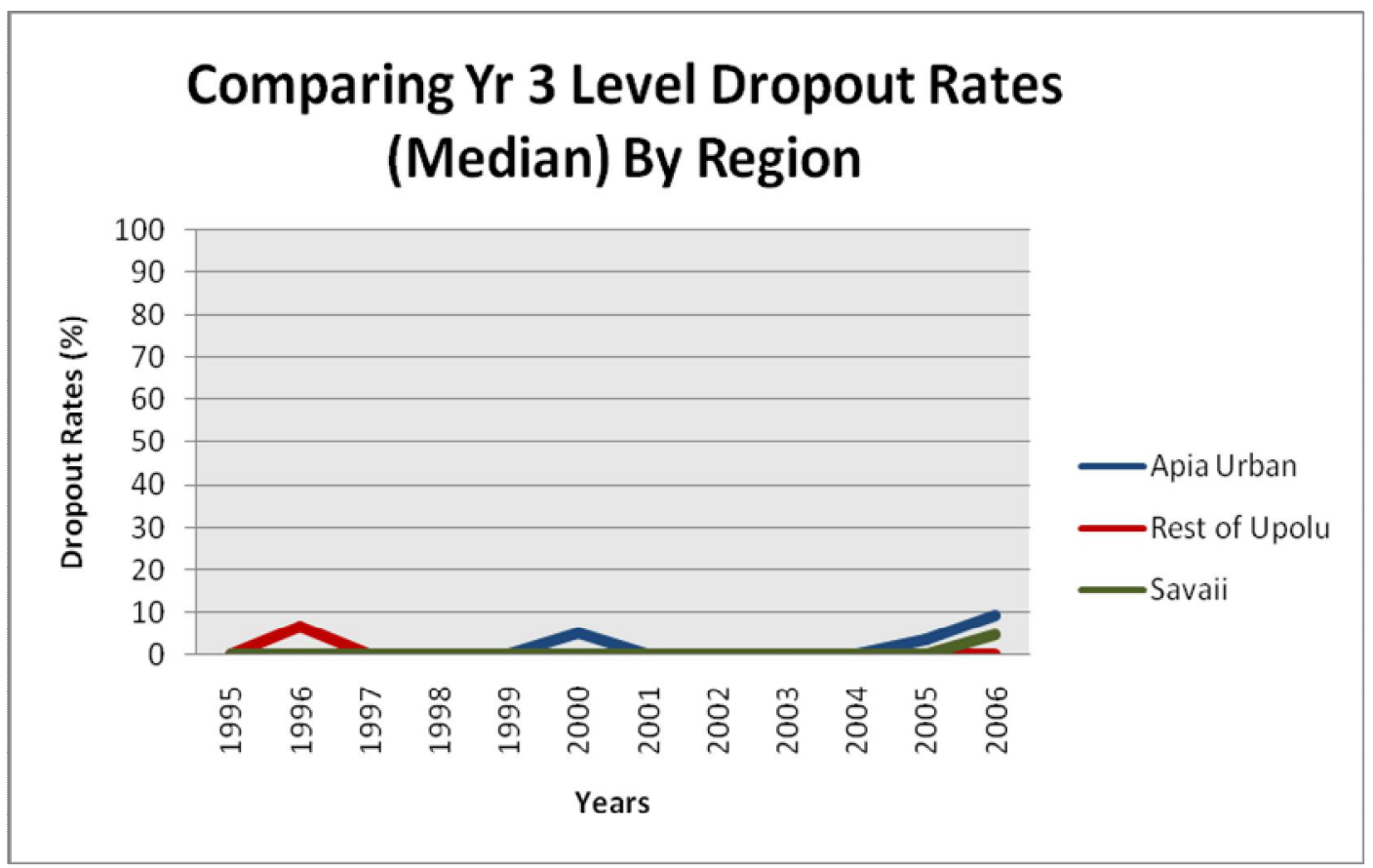

C. RDR. G2: Comparing Yr 4 Level Dropout Rates by Region

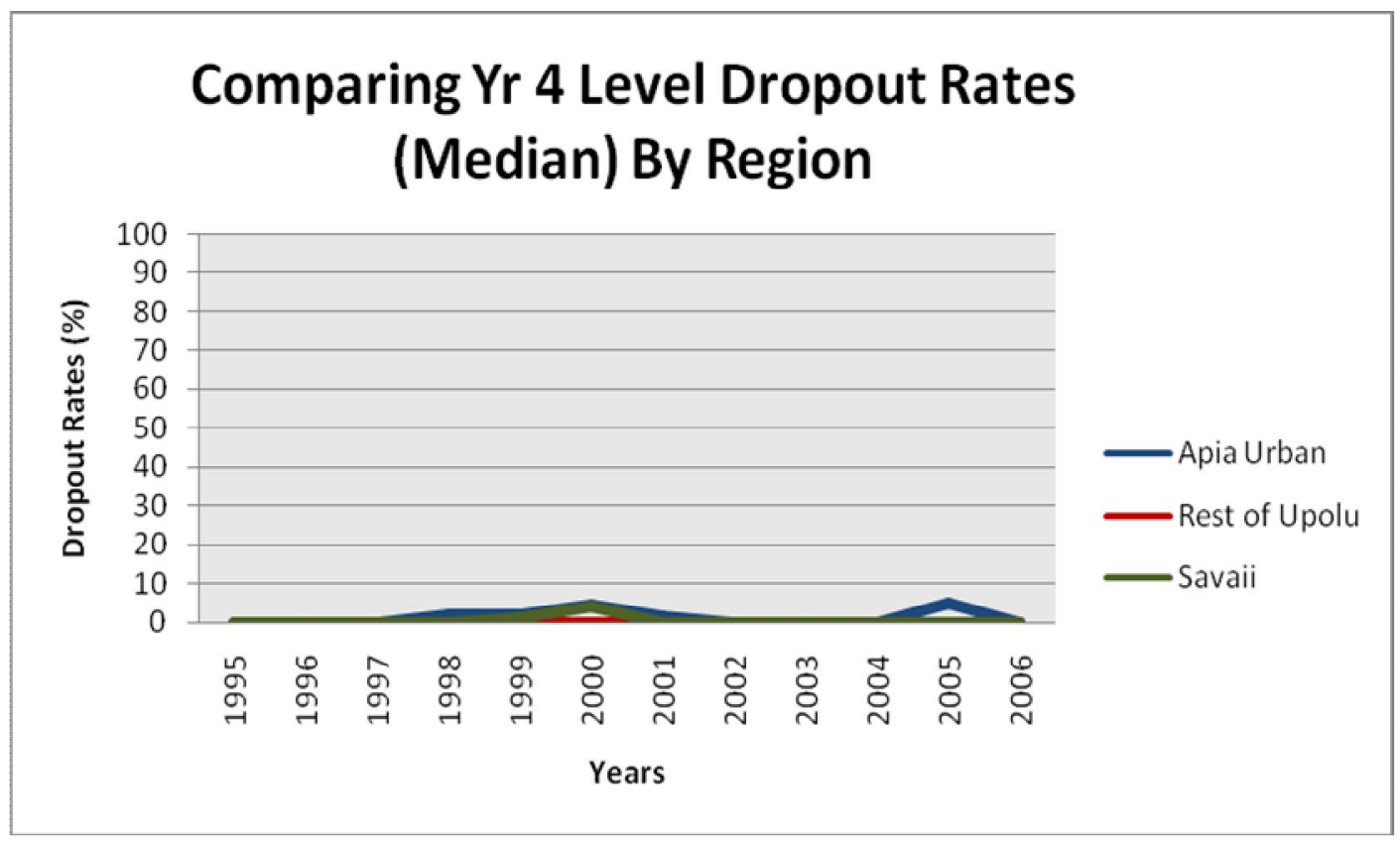


C. RDR. G3: Comparing Yr 5 Level Dropout Rates by Region

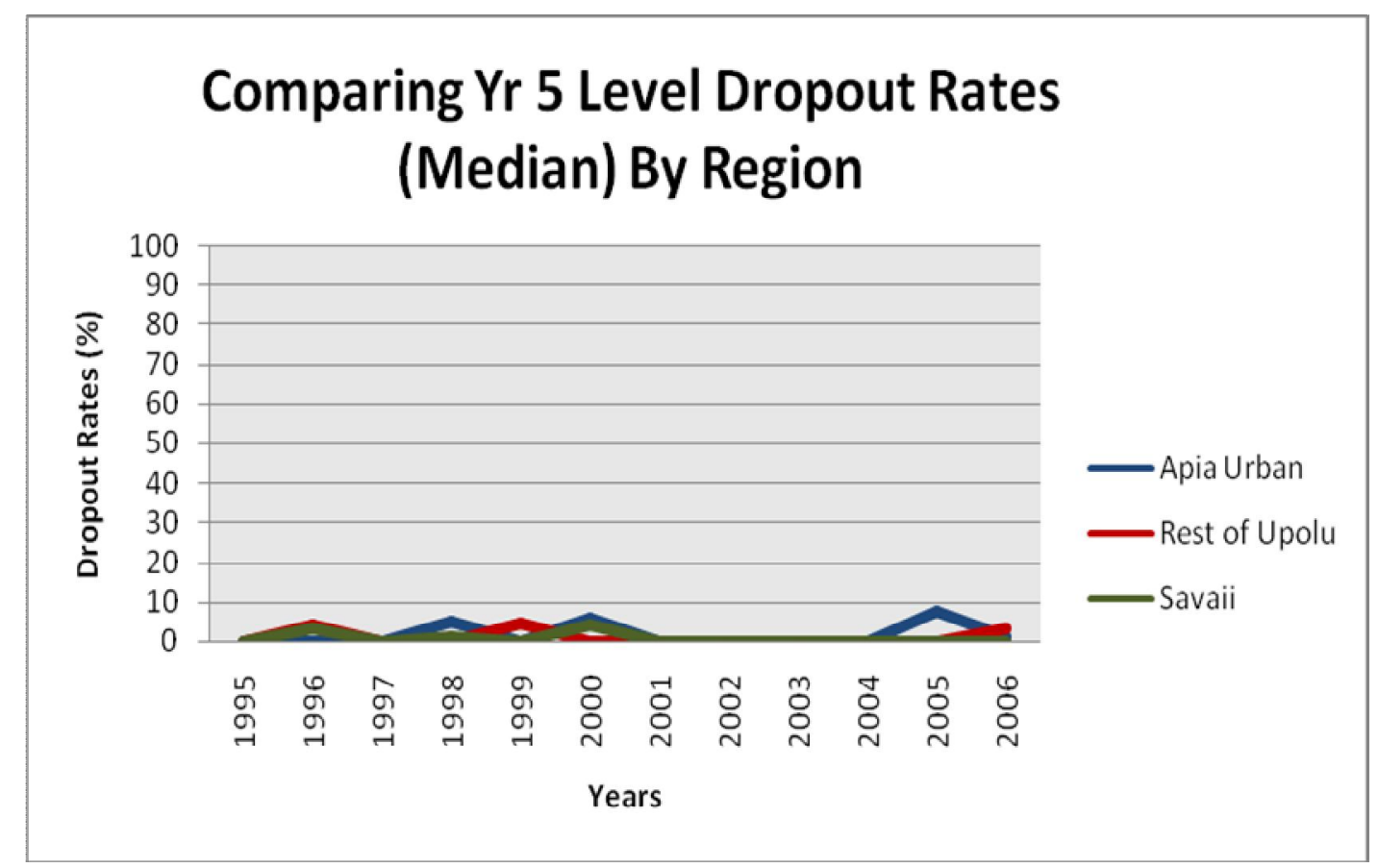

C. RDR. G4: Comparing Yr 6 Level Dropout Rates by Region

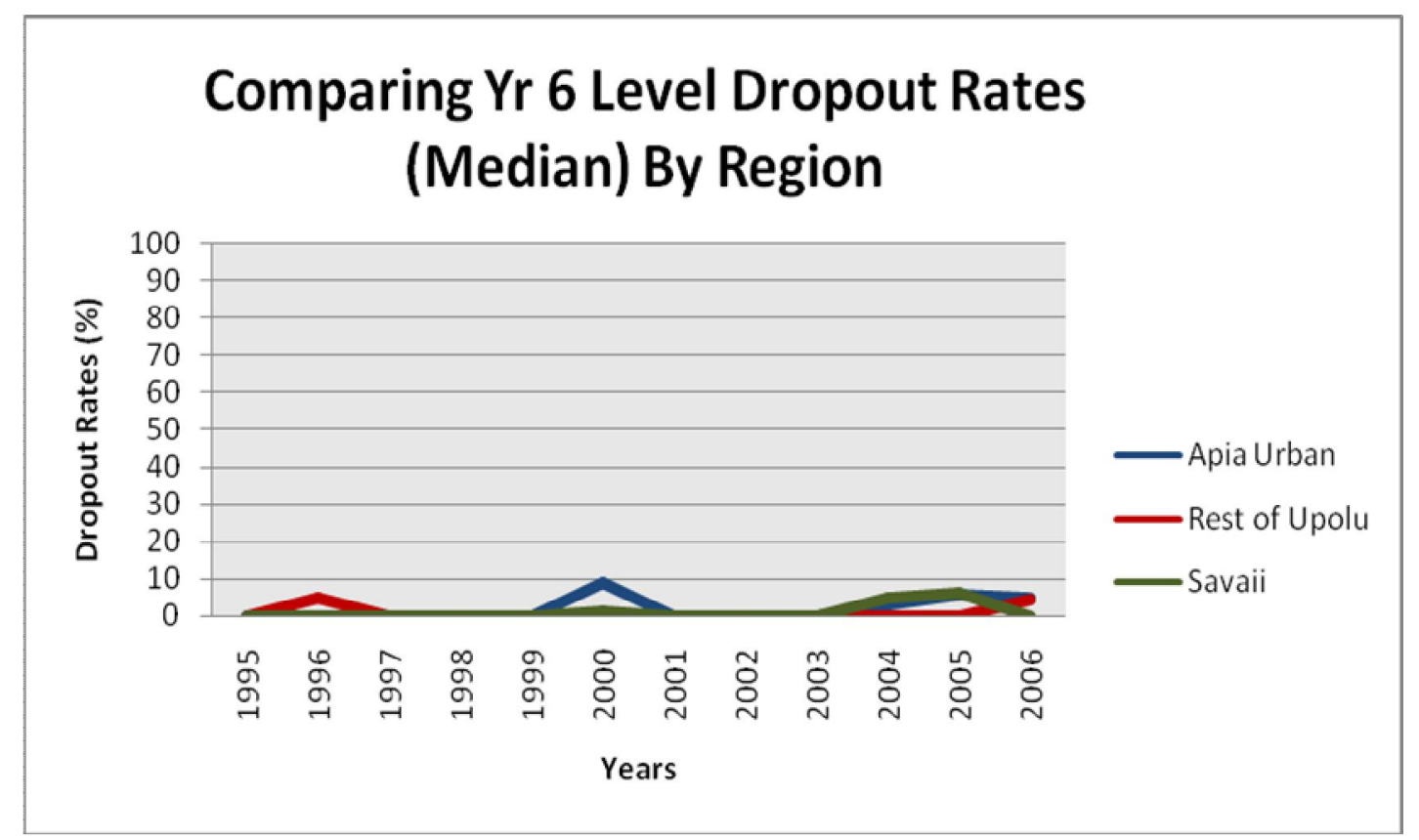


C. RDR. G5: Comparing Yr 8 Level Dropout Rates by Region

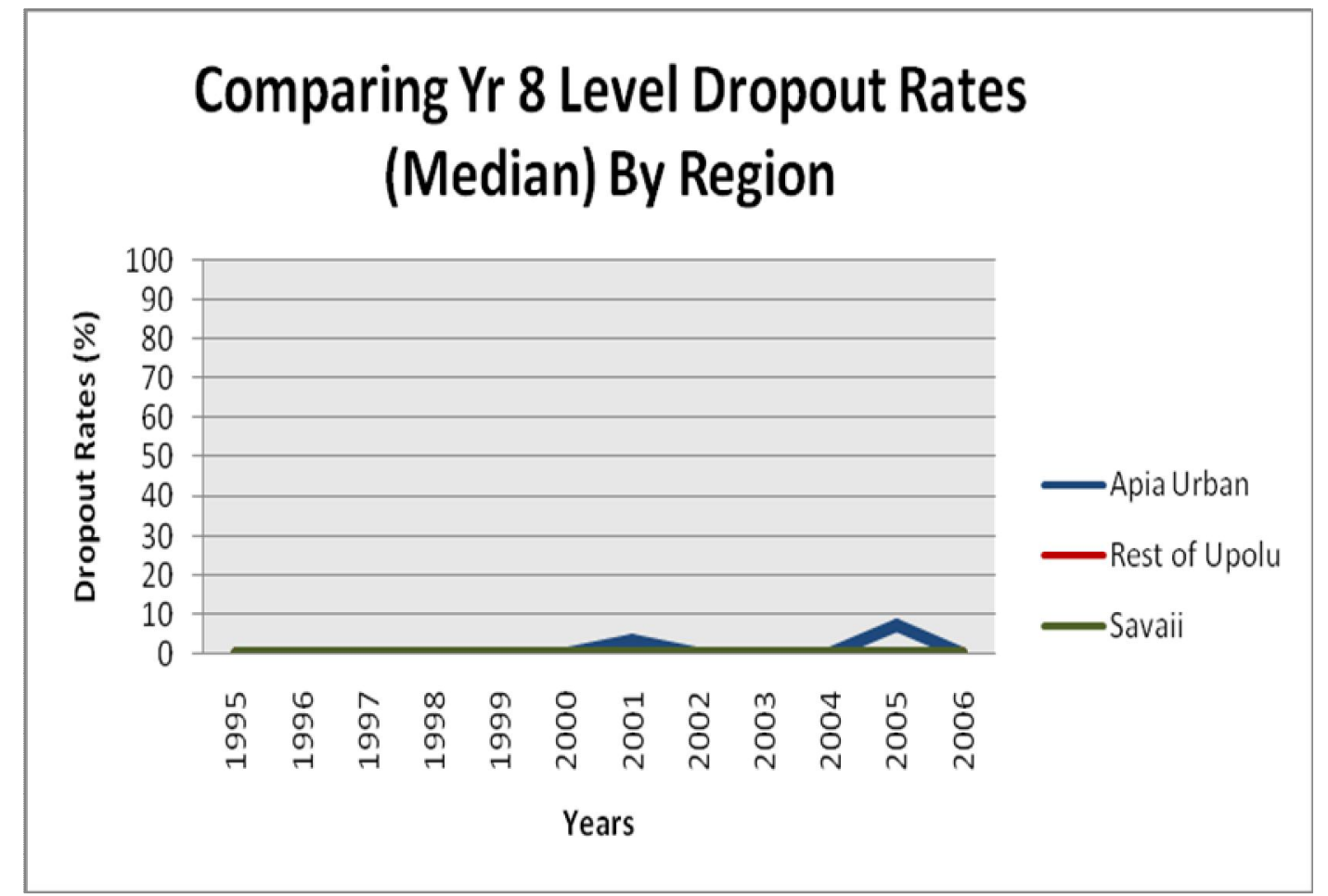

C. SSDR. G1: Comparing Yr 3 Level Dropout Rates by School Status

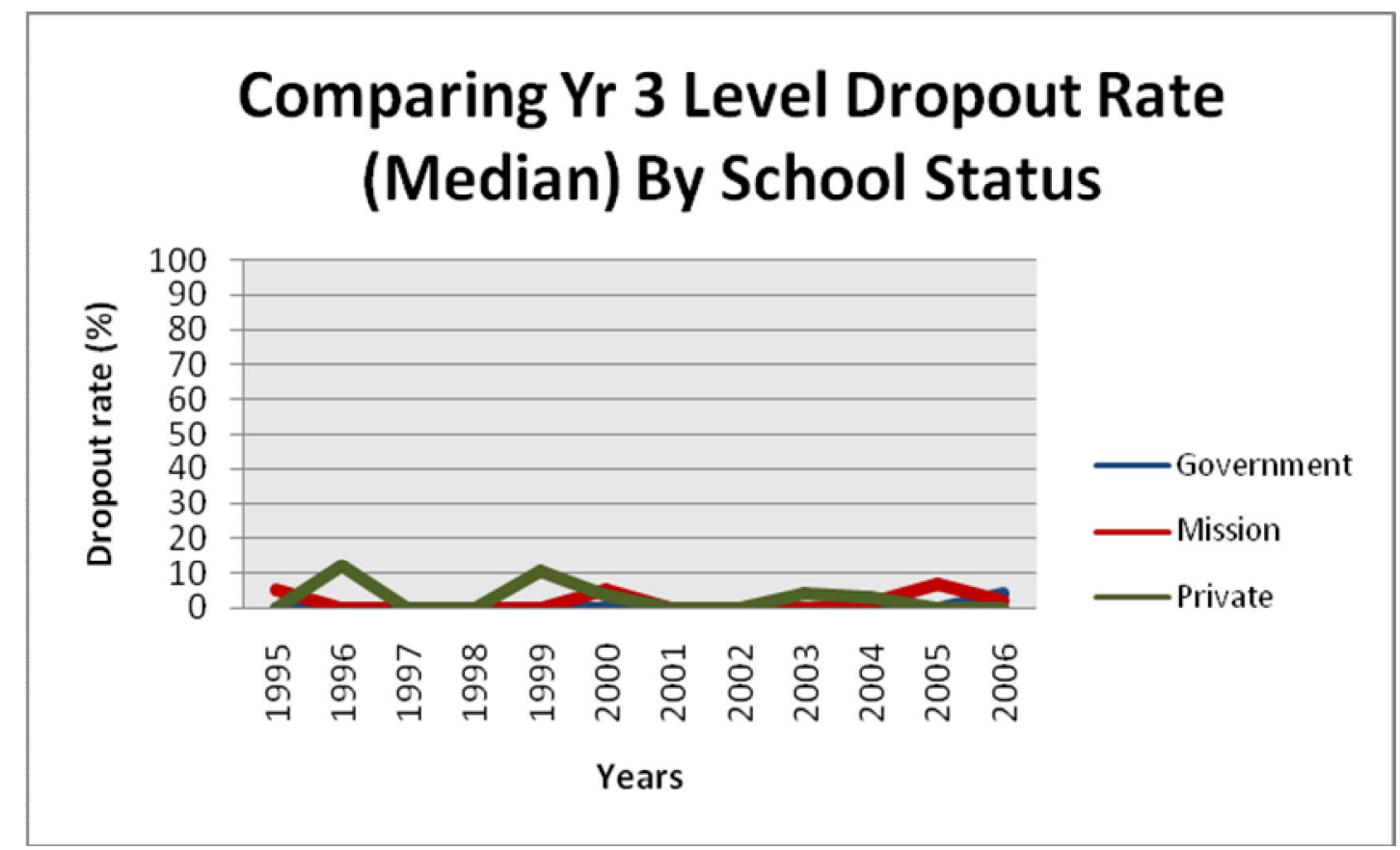


C. SSDR. G2: Comparing Yr 4 Level Dropout Rates by School Status

\section{Comparing Yr 4 Level Dropout Rate (Median) By School Status}

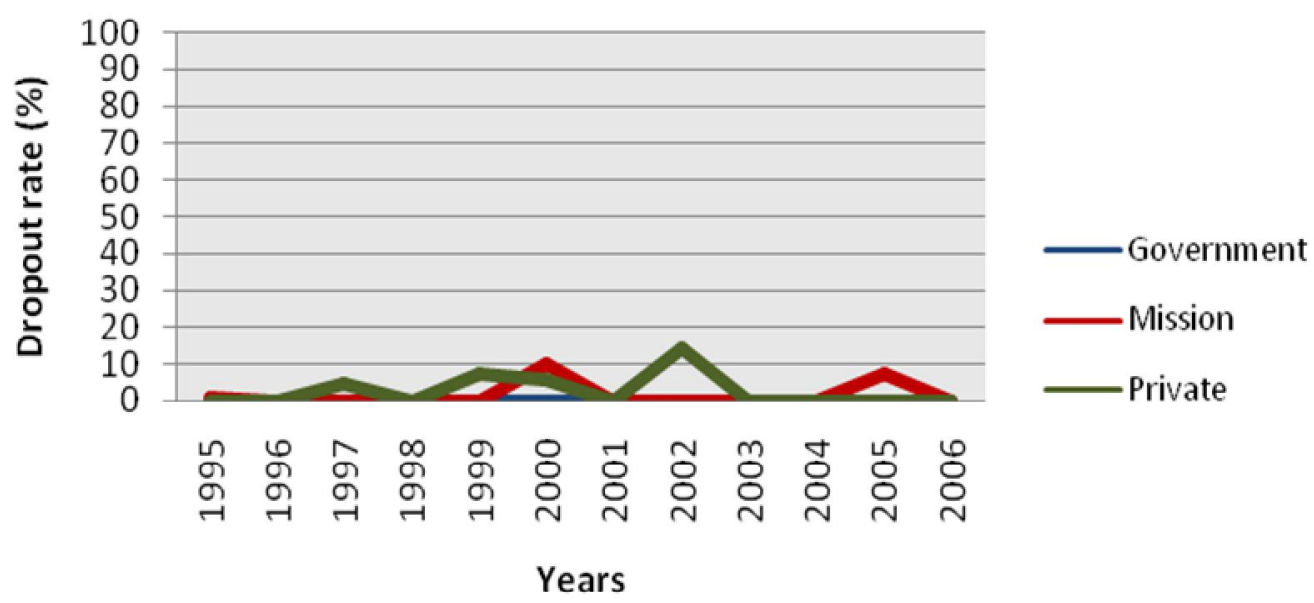

C. SSDR. G3: Comparing Yr 5 Level Dropout Rates by School Status

\section{Comparing Yr 5 Level Dropout Rate (Median) By School Status}

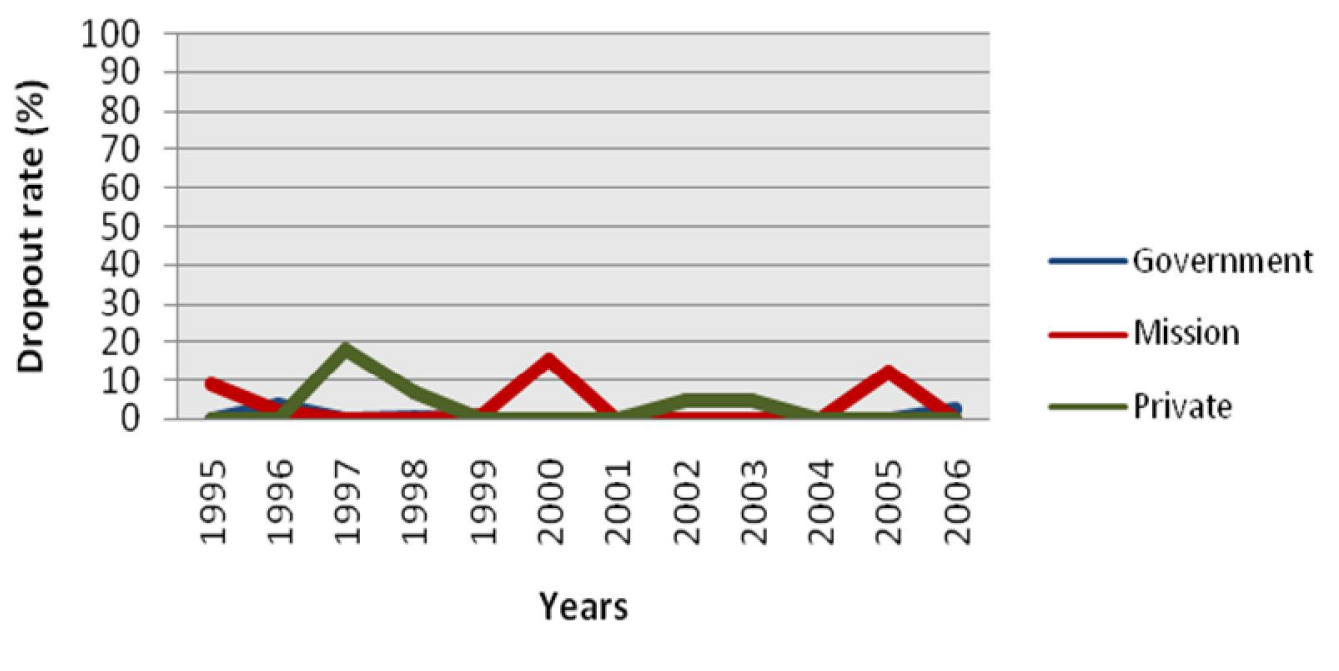


C. SSDR. G4: Comparing Yr 6 Level Dropout Rates by School Status

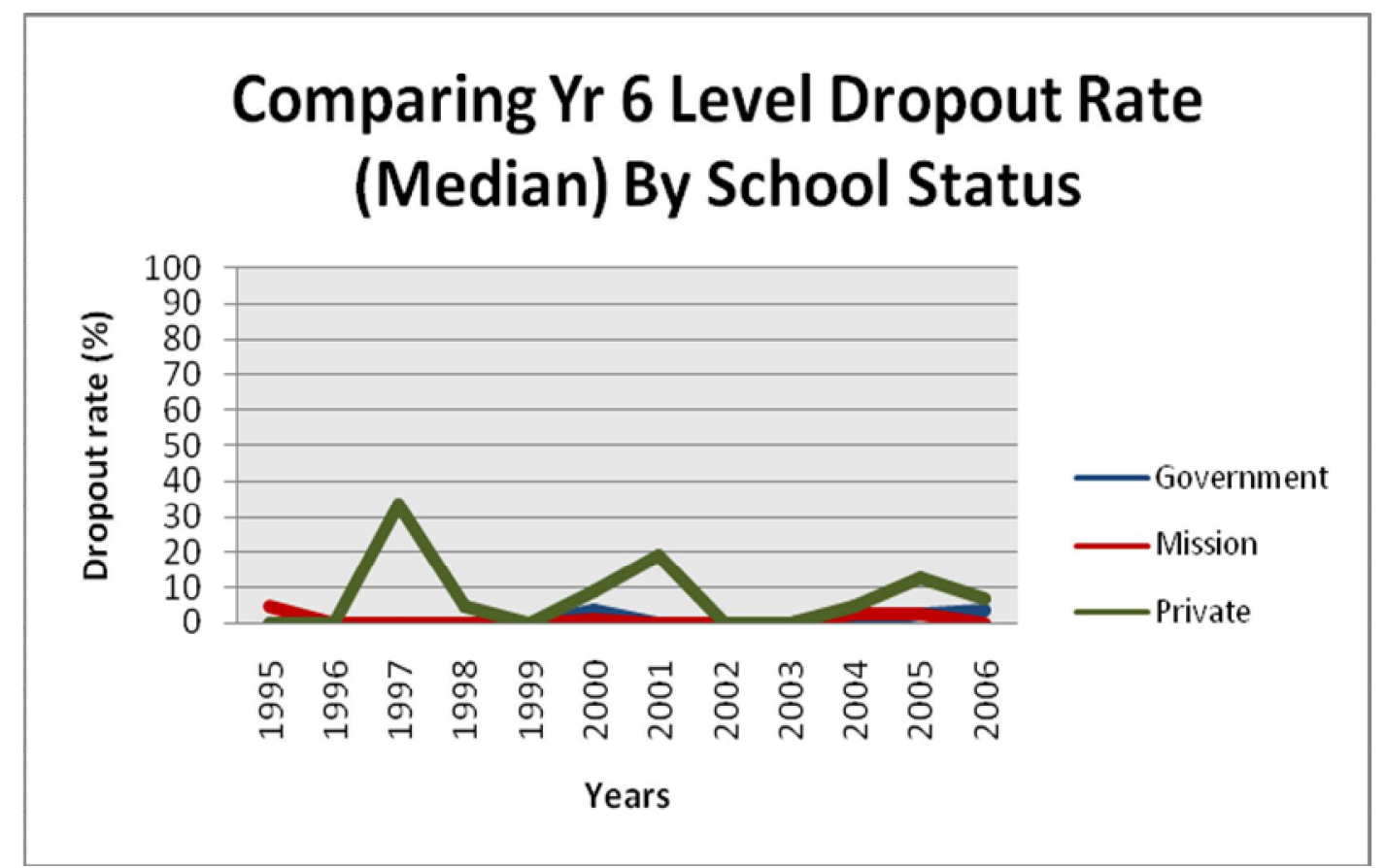

C. SSDR. G5: Comparing Yr 7 Level Dropout Rates by School Status

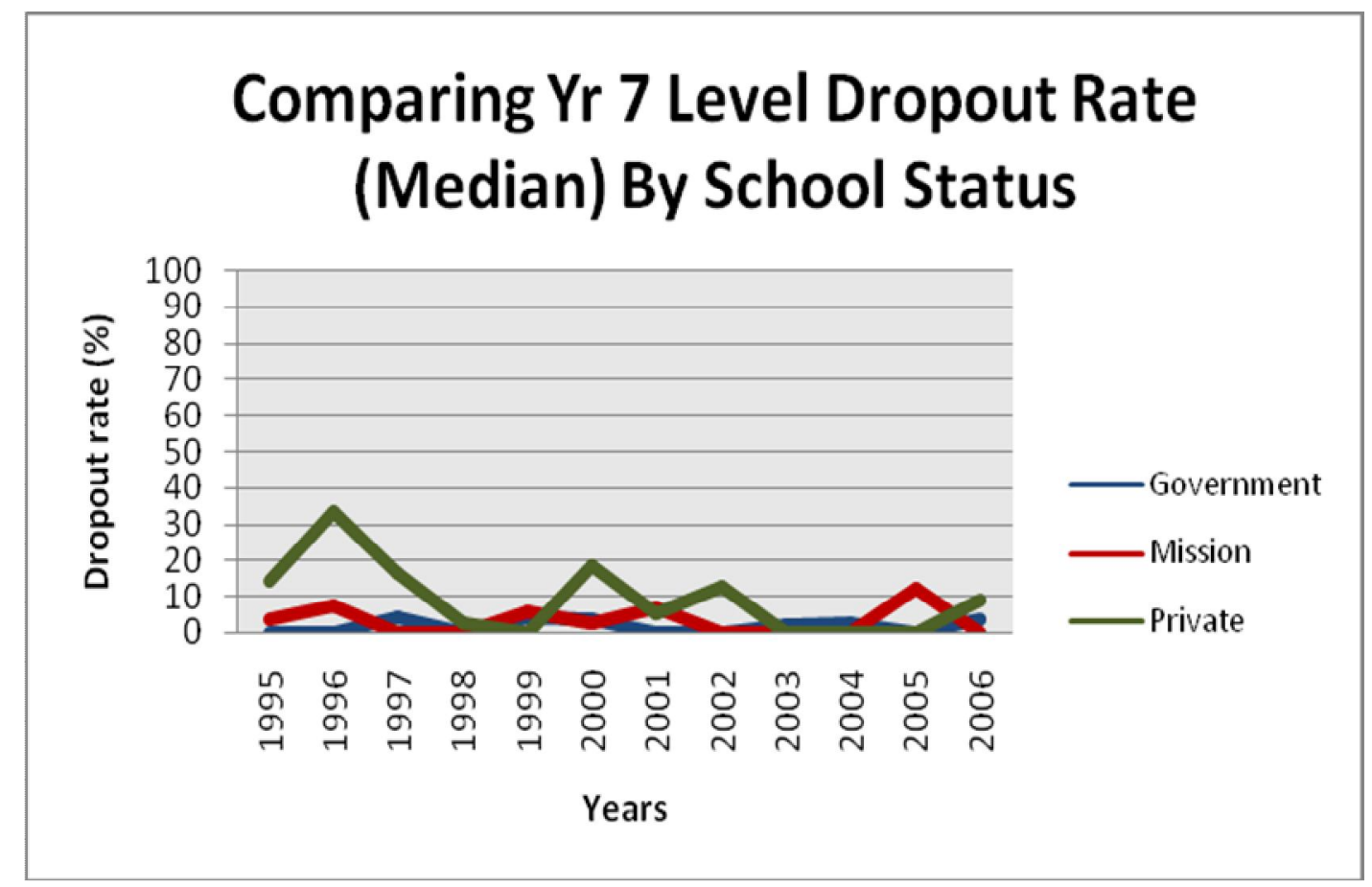


C. SSDR. G6: Comparing Yr 8 Level Dropout Rates by School Status

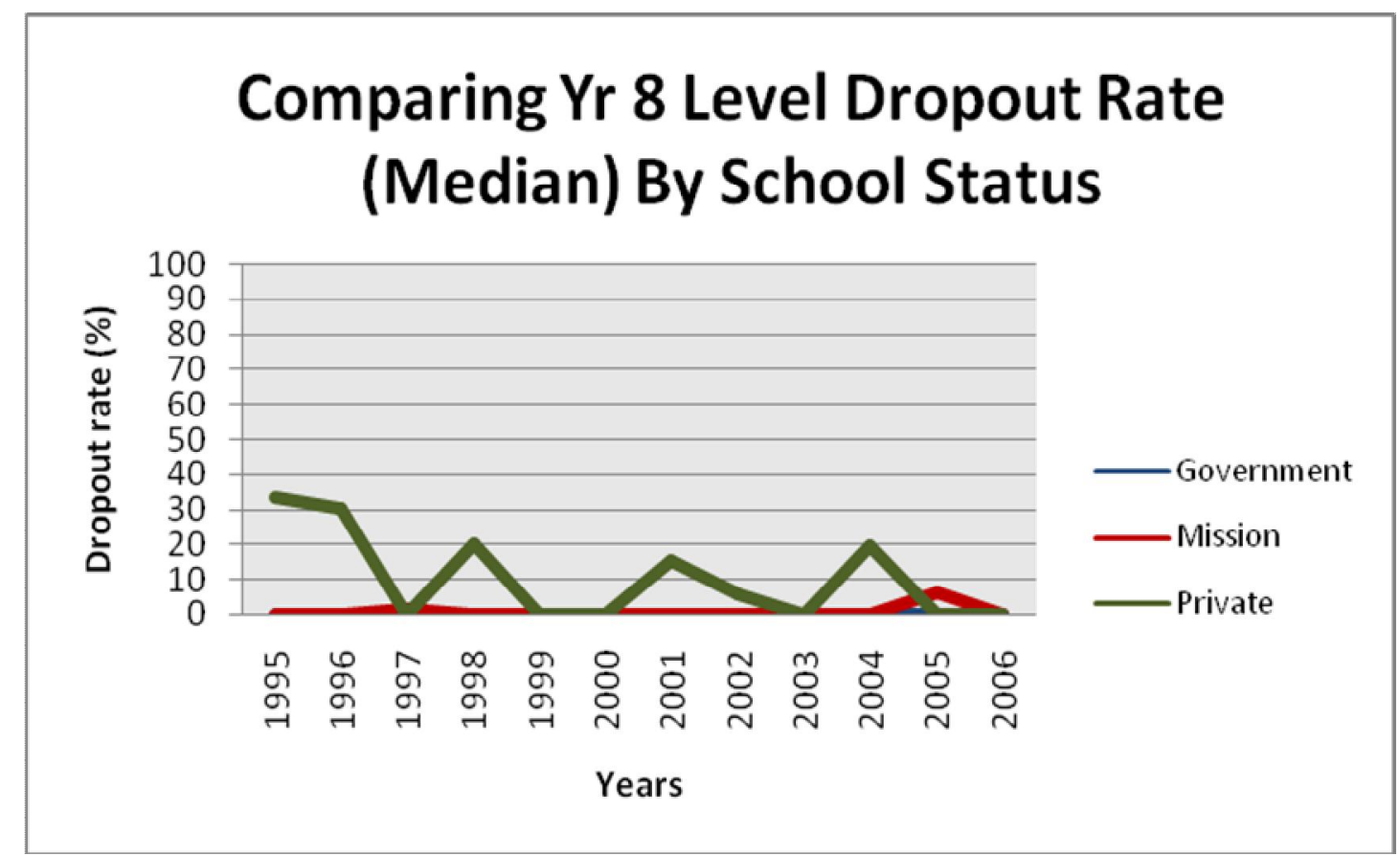

C. STR. RG1: Distribution of the Student-Teacher Ratio by Region (1997)

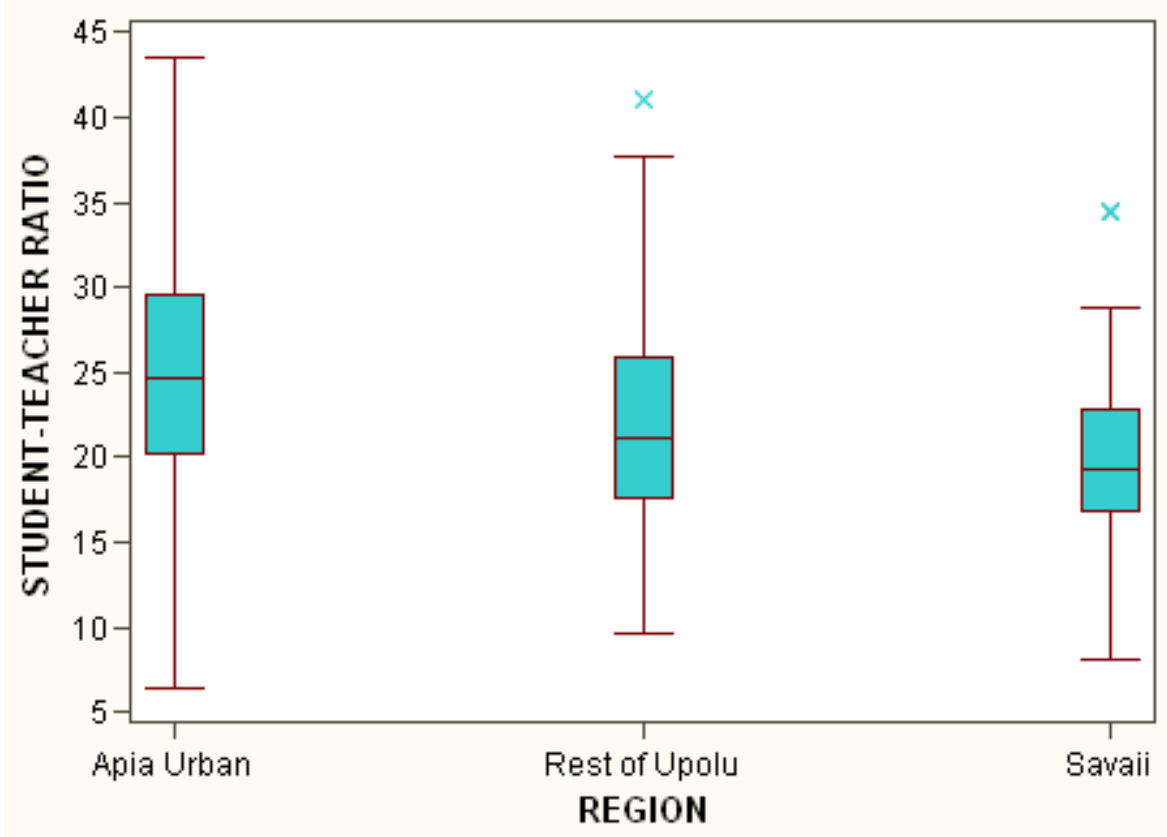


C. STR. RG2: Distribution of the Student-Teacher Ratio by Region (1998)

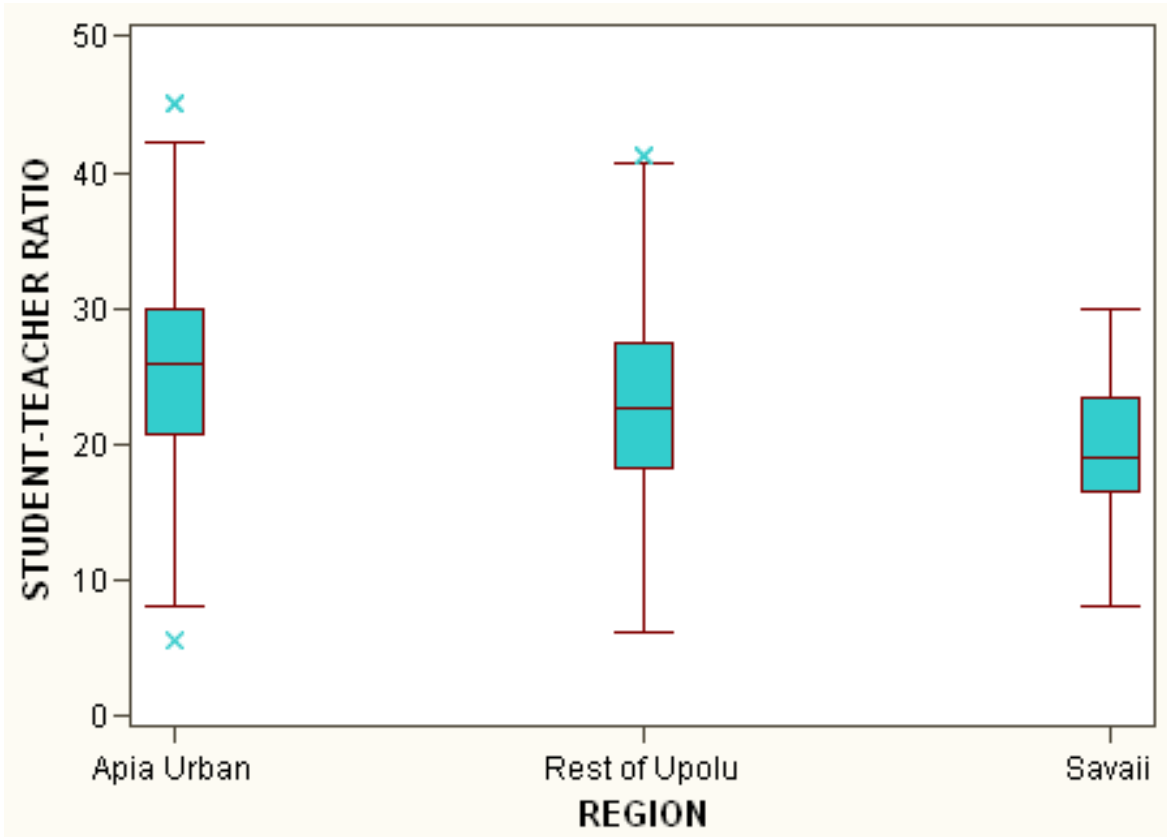

C. STR. RG3: Distribution of the Student-Teacher Ratio by Region (1999)

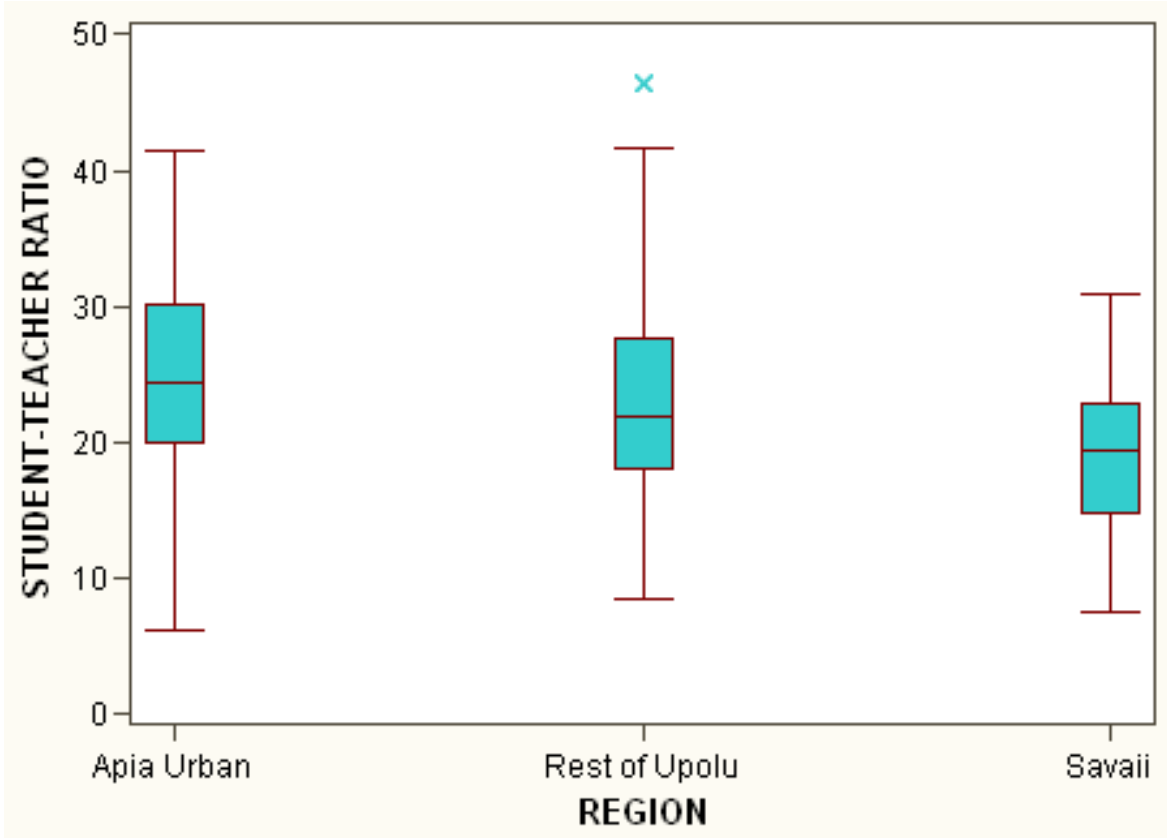


C. STR. RG4: Distribution of the Student-Teacher Ratio by Region (2000)

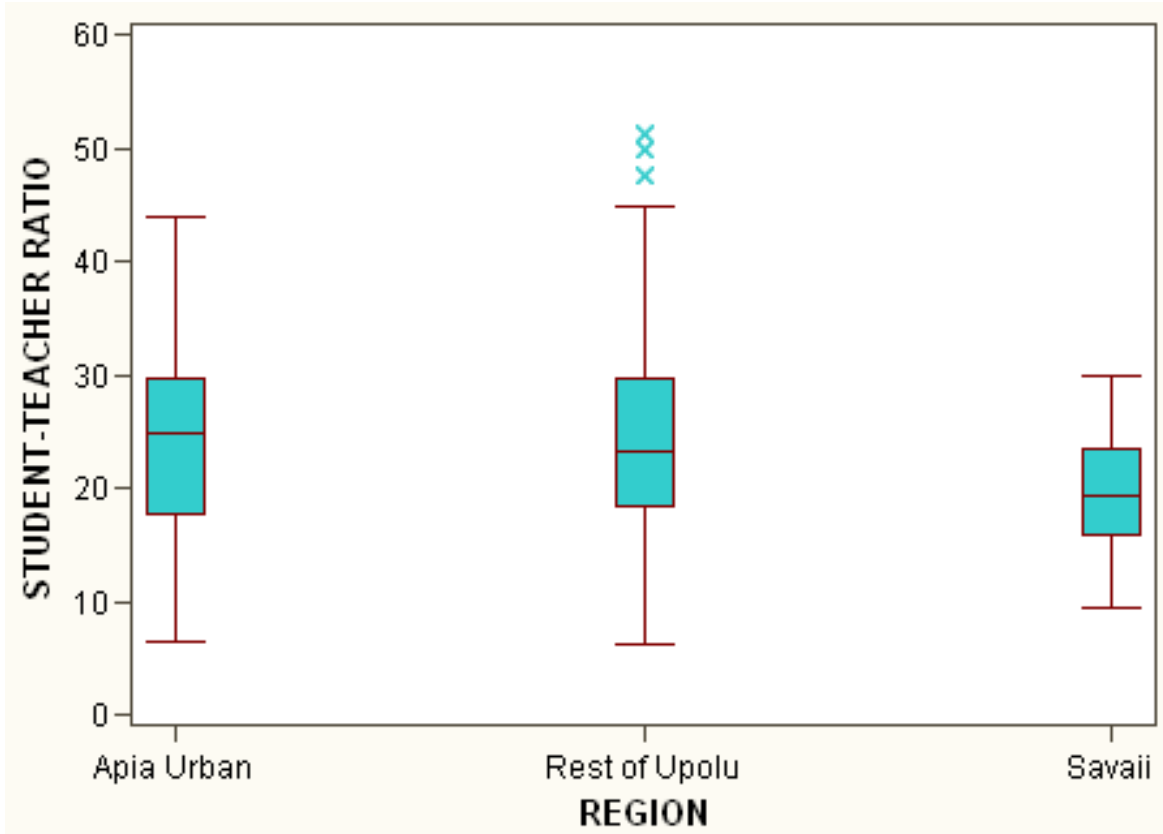

C. STR. RG5: Distribution of the Student-Teacher Ratio by Region (2001)

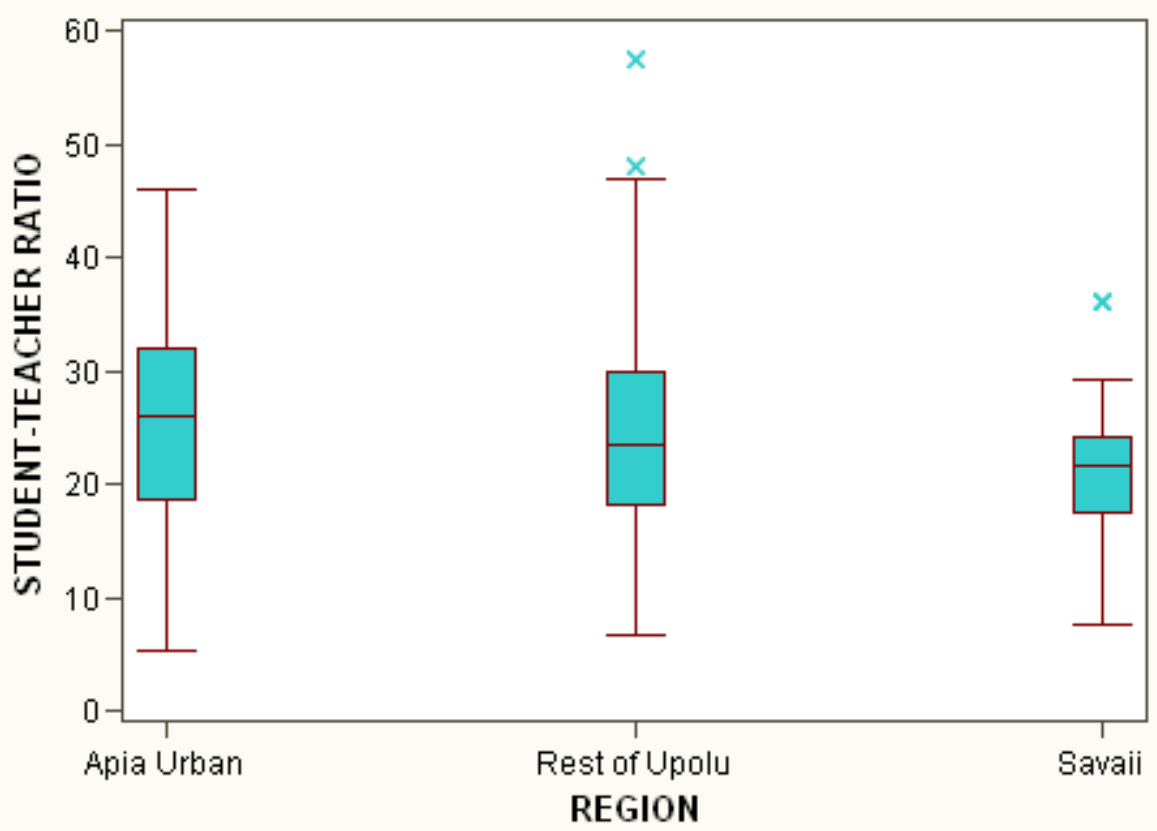


C. STR. RG6: Distribution of the Student-Teacher Ratio by Region (2002)

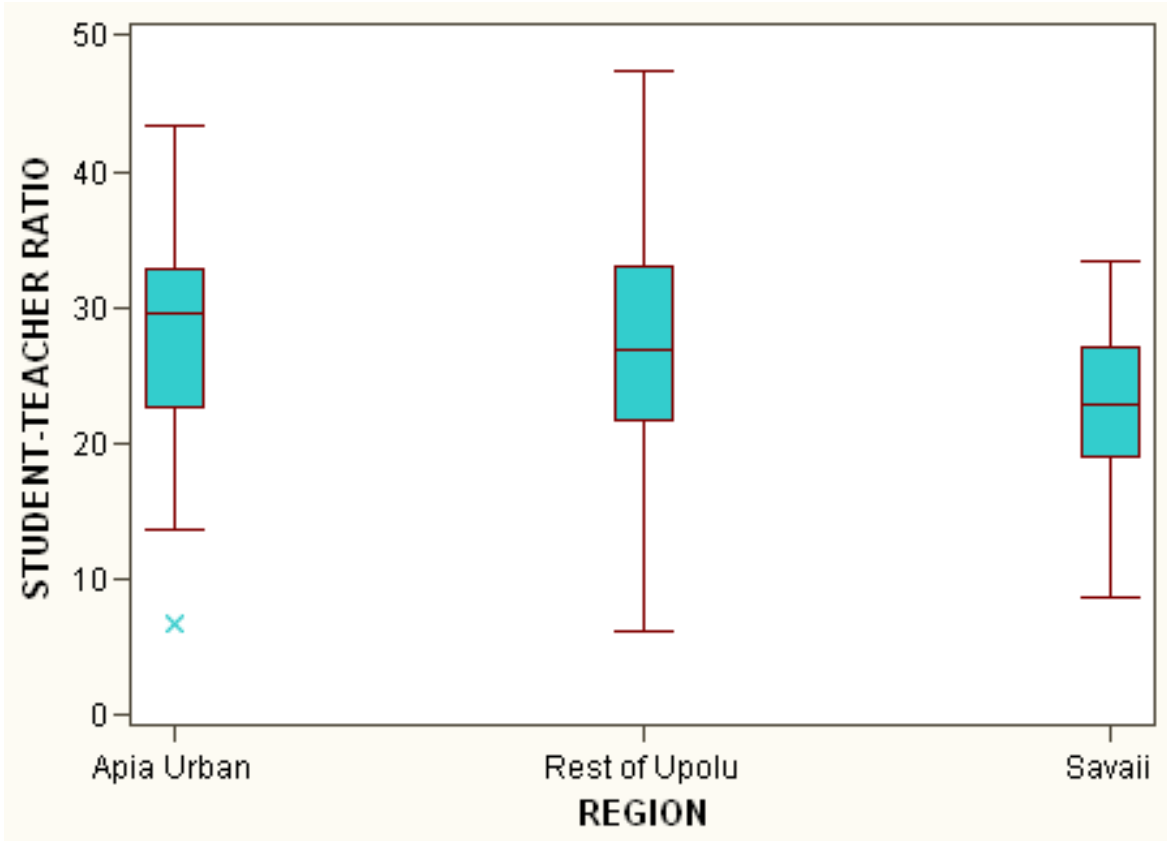

C. STR. RG7: Distribution of the Student-Teacher Ratio by Region (2003)

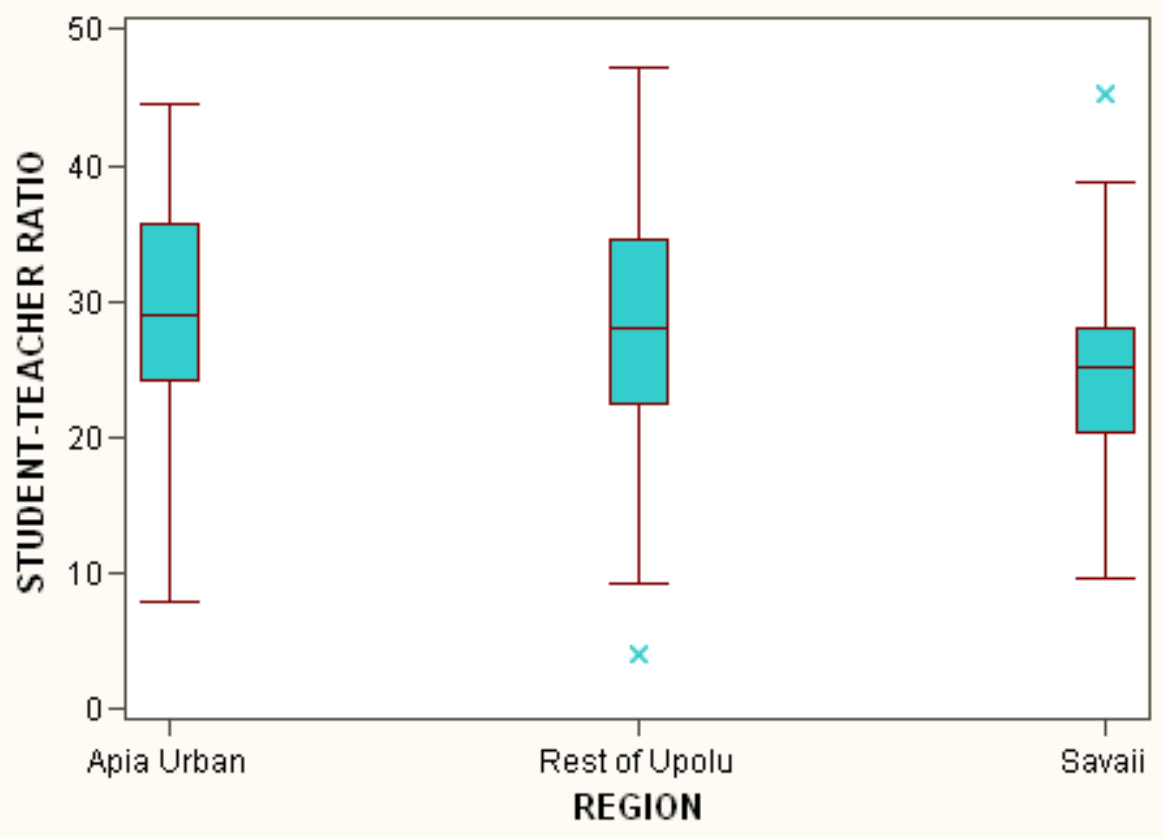


C. STR. RG8: Distribution of the Student-Teacher Ratio by Region (2004)

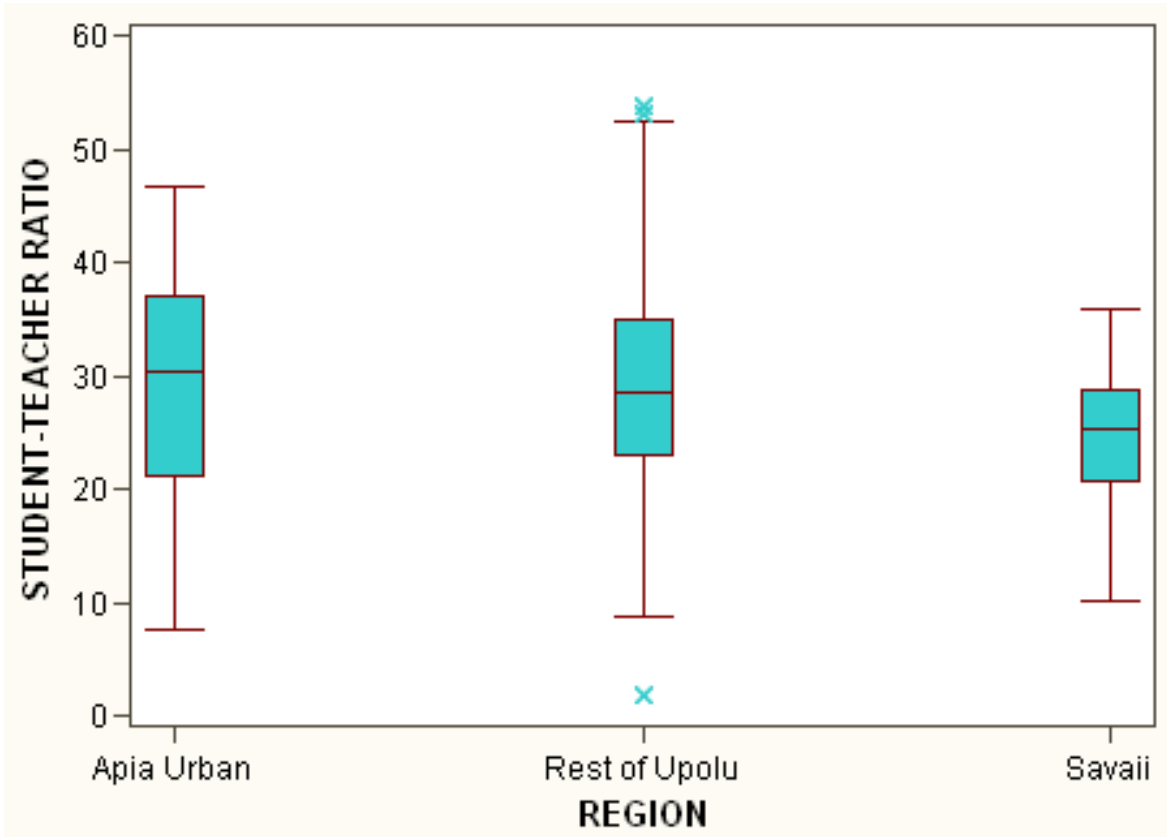

C. STR. RG9: Distribution of the Student-Teacher Ratio by Region (2005)

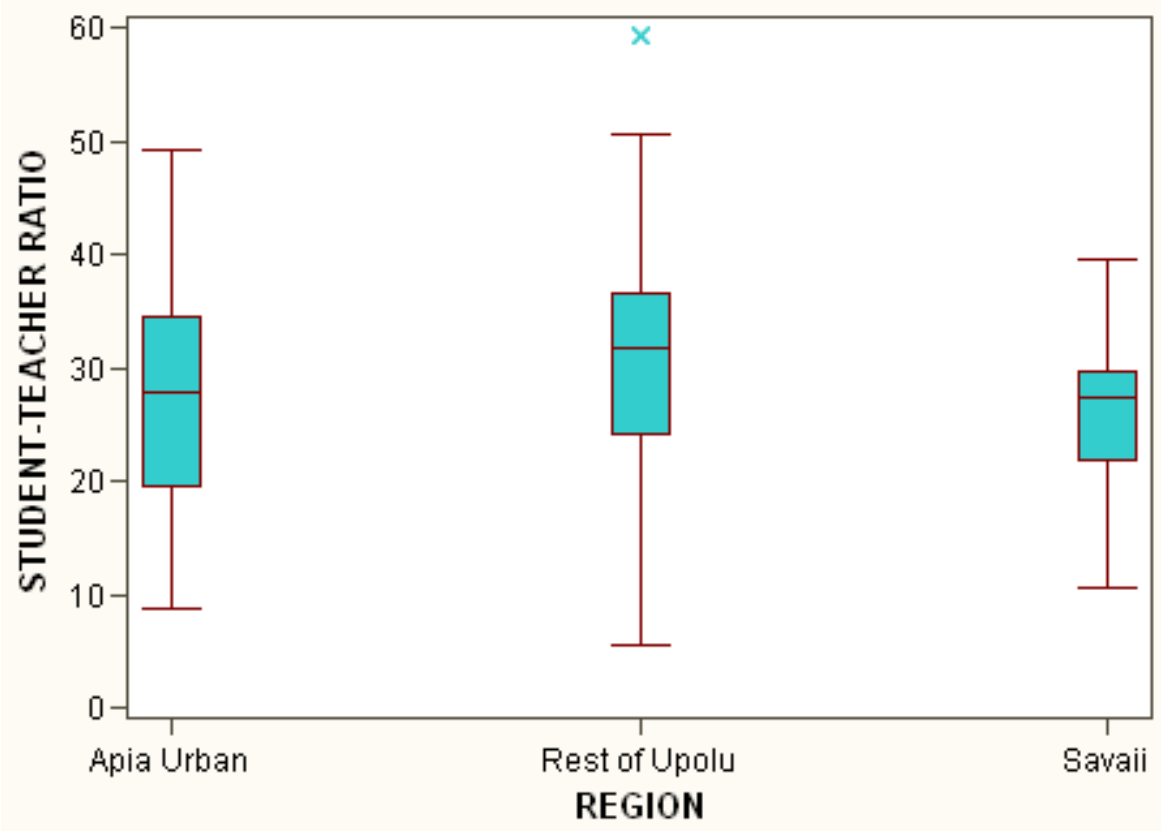


C. STR. RG10: Distribution of the Student-Teacher Ratio by Region (2006)

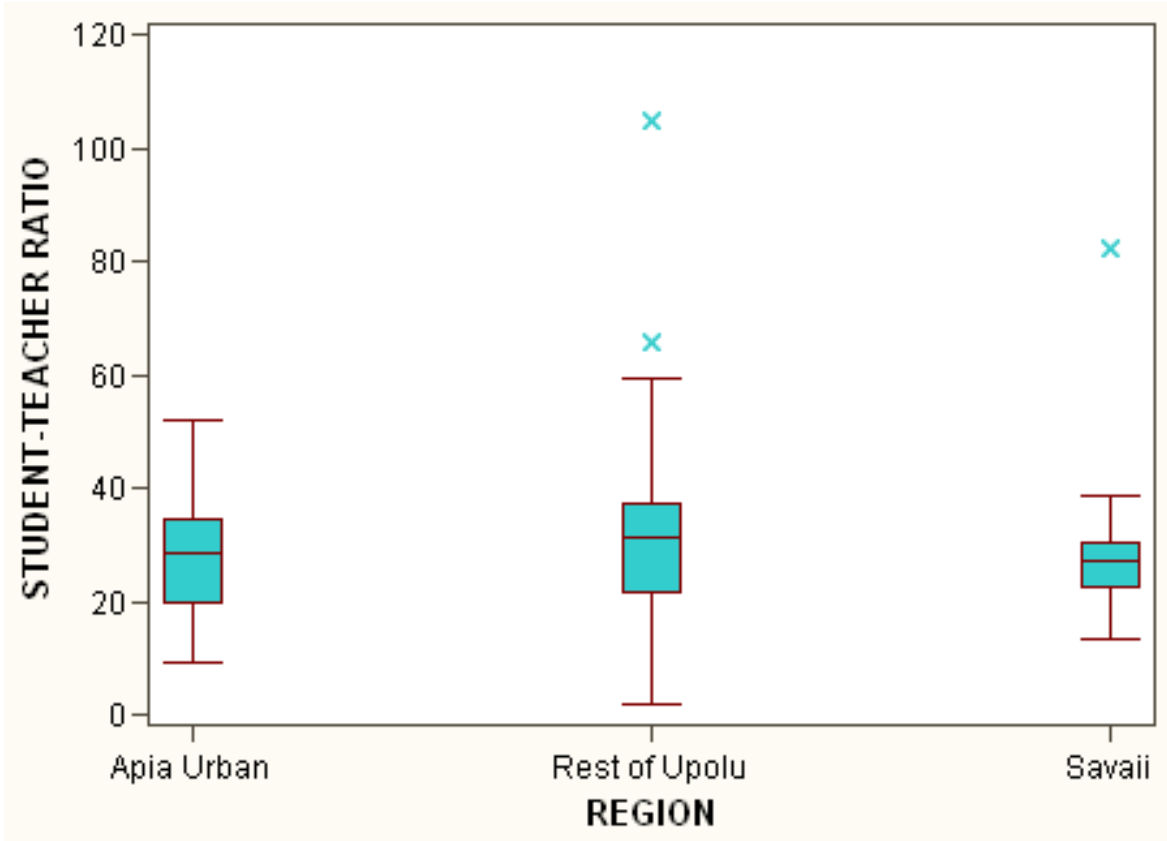

C. STR. SSG1: Distribution of the Student-Teacher Ratio by School Status (1995)

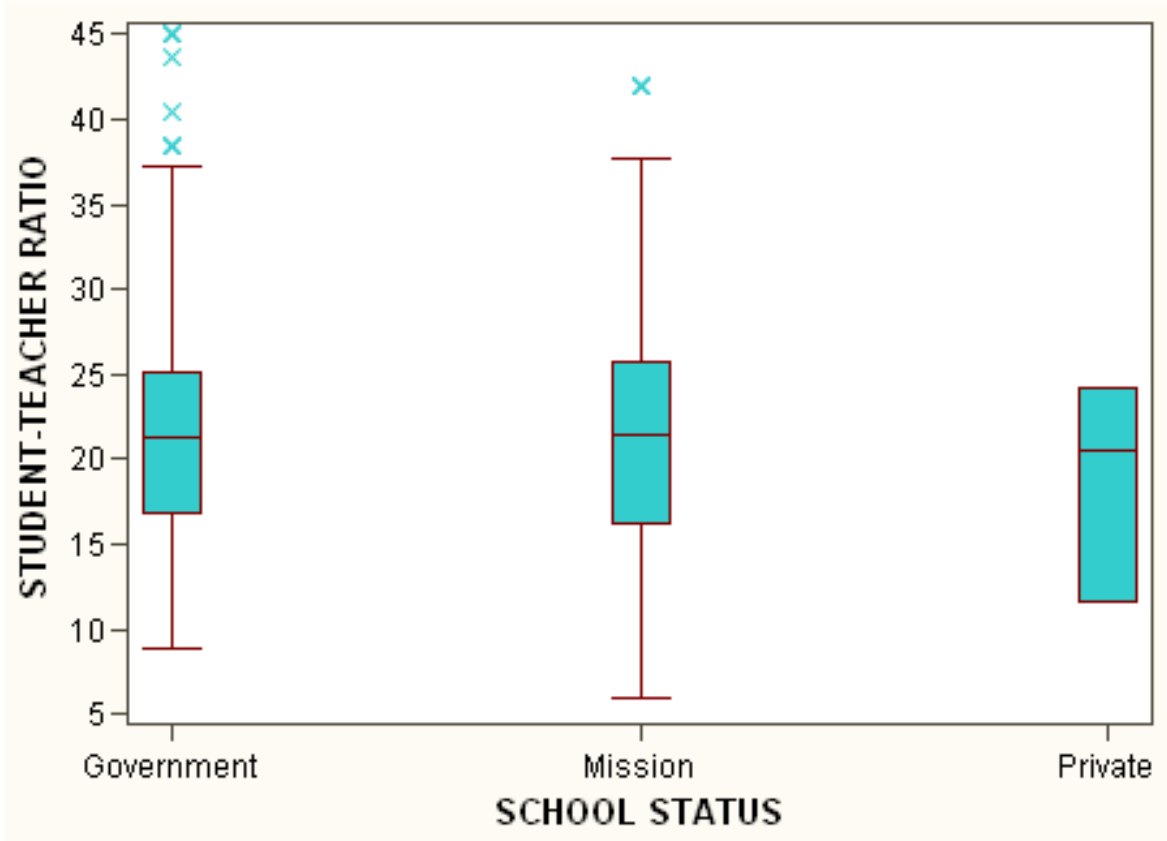


C. STR. SSG2: Distribution of the Student-Teacher Ratio by School Status (1996)

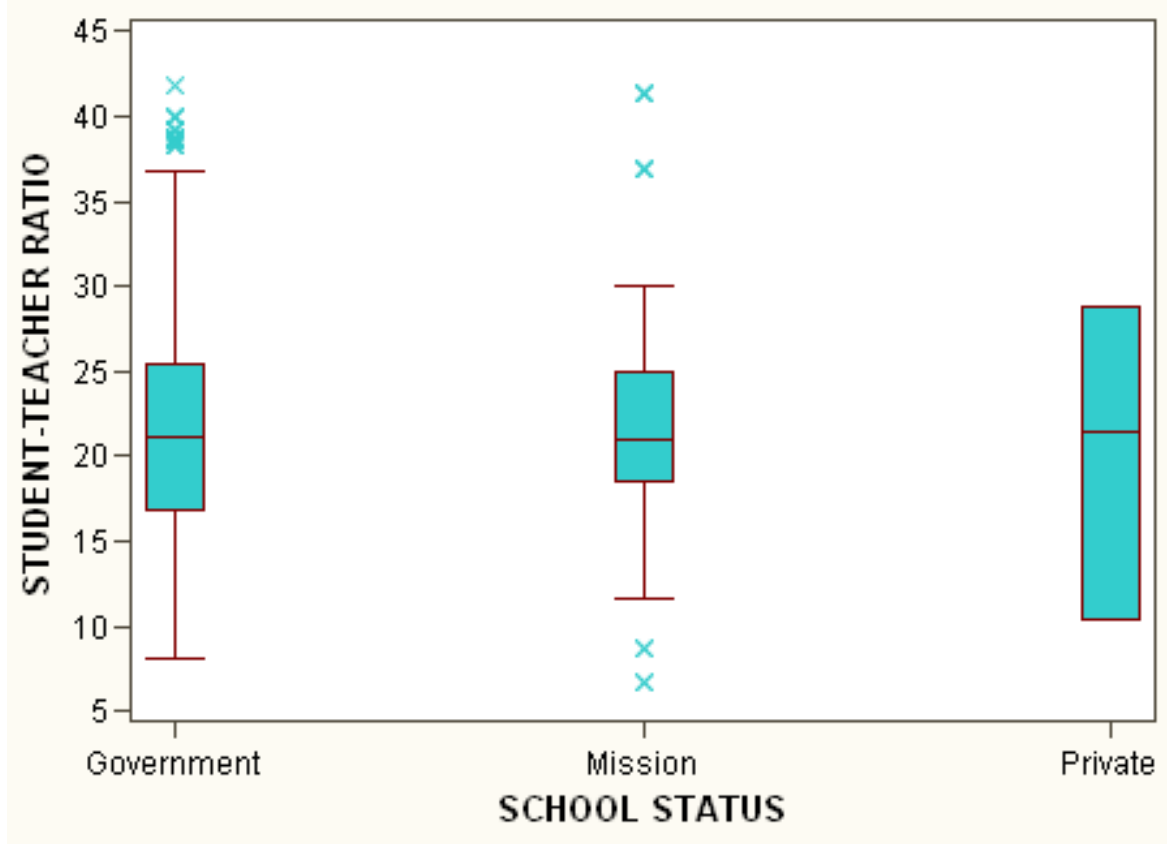

C. STR. SSG3: Distribution of the Student-Teacher Ratio by School Status (1997)

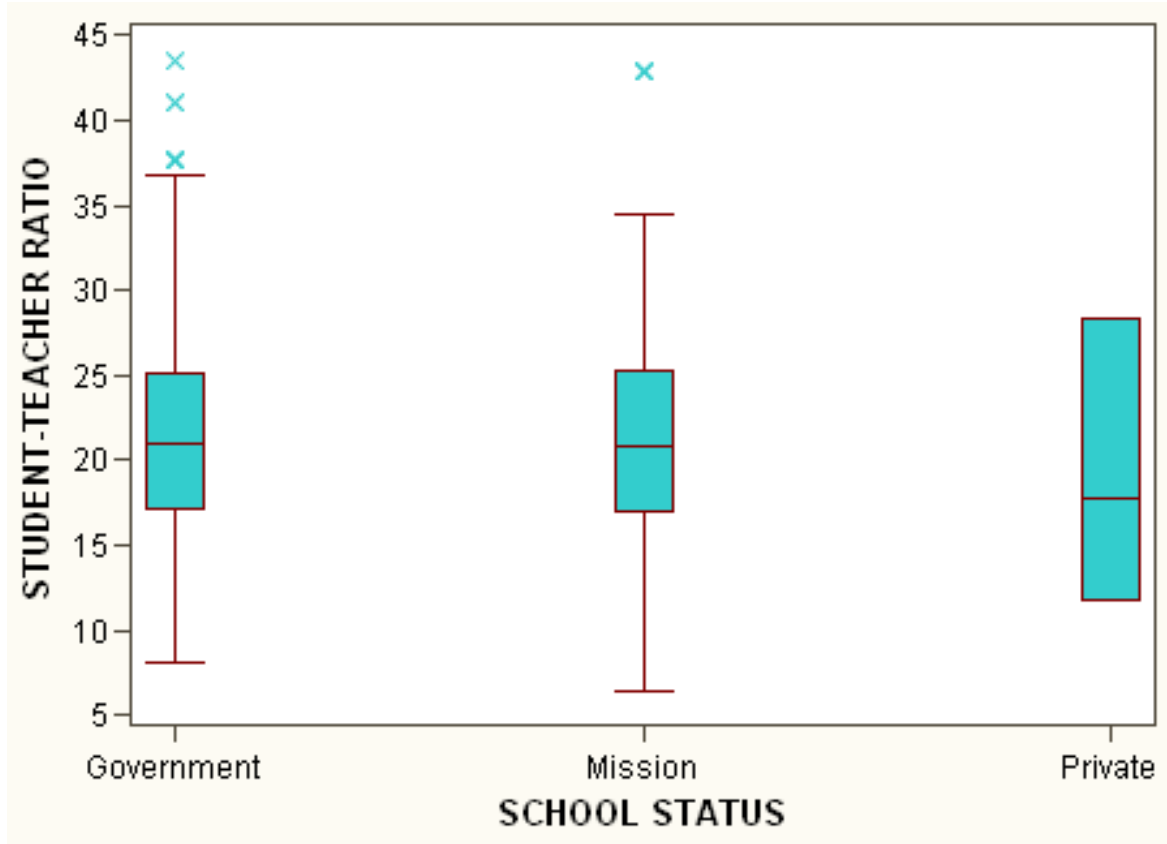


C. STR. SSG4: Distribution of the Student-Teacher Ratio by School Status

(1998)

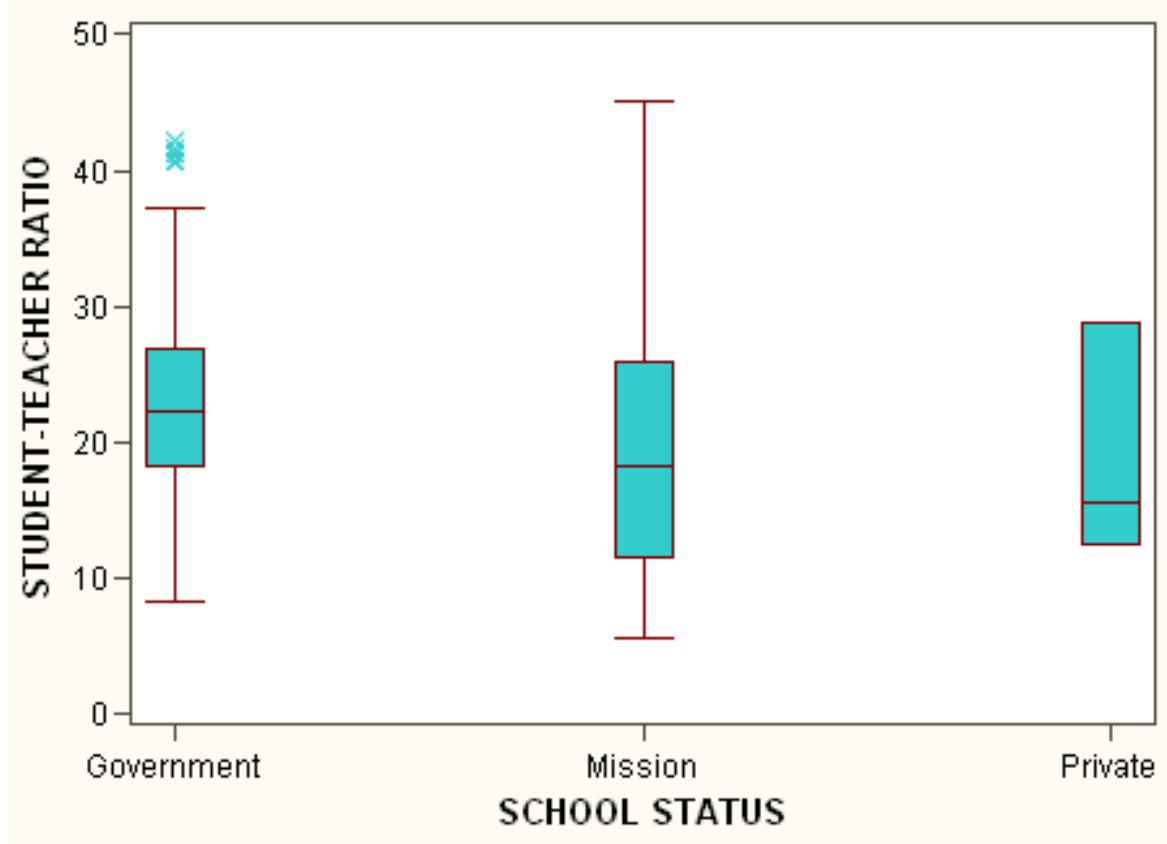

C. STR. SSG5: Distribution of the Student-Teacher Ratio by School Status (1999)

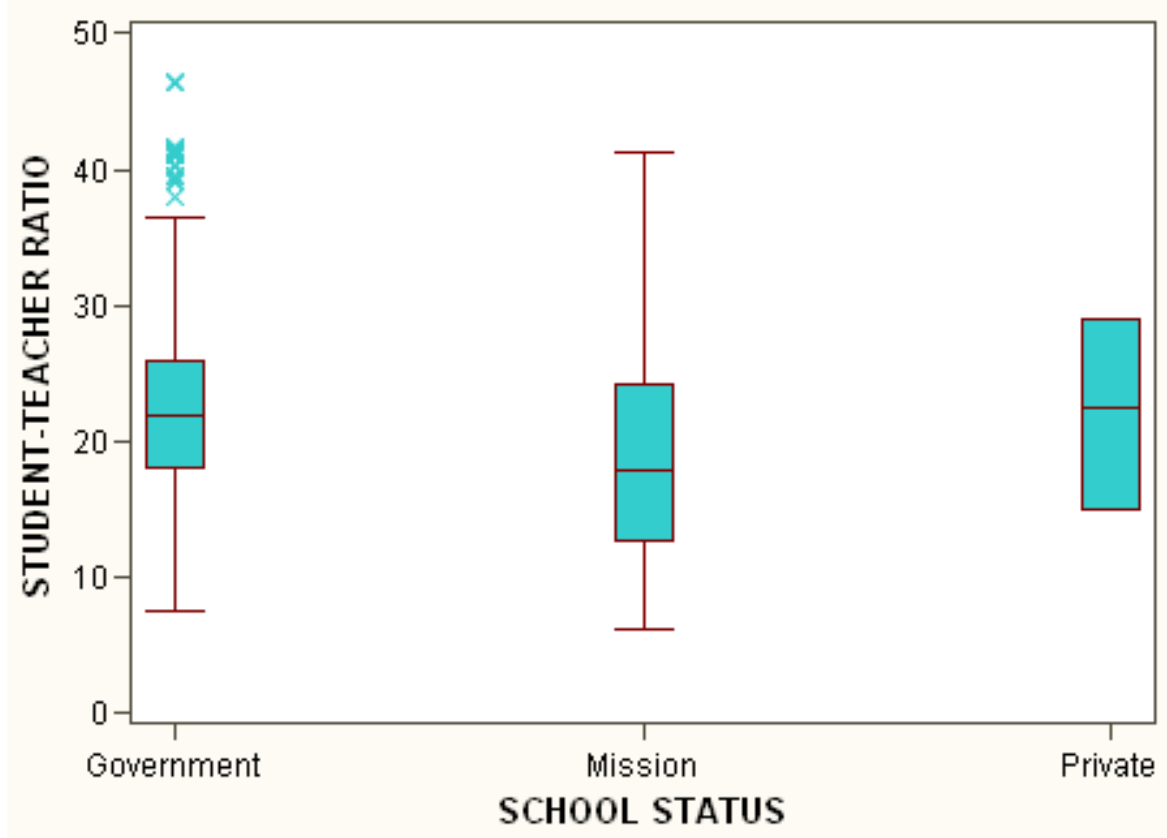


C. STR. SSG6: Distribution of the Student-Teacher Ratio by School Status (2000)

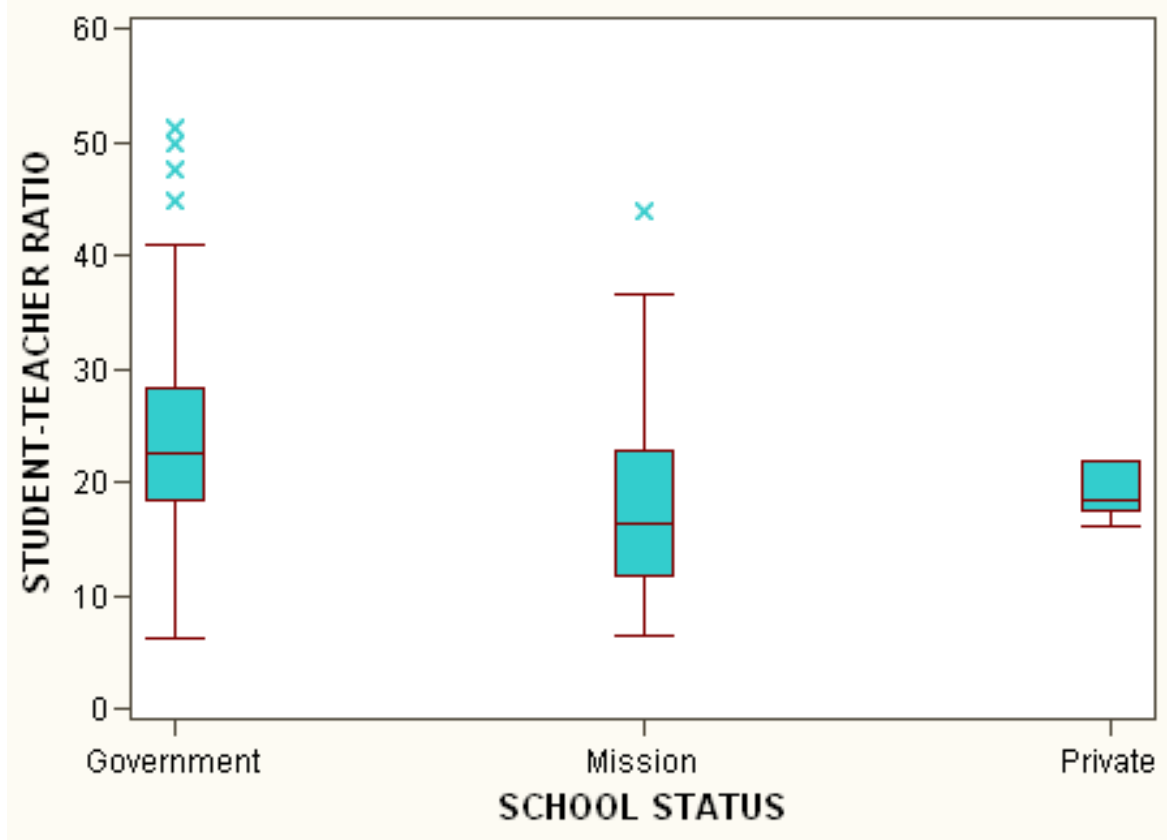

C. STR. SSG7: Distribution of the Student-Teacher Ratio by School Status (2001)

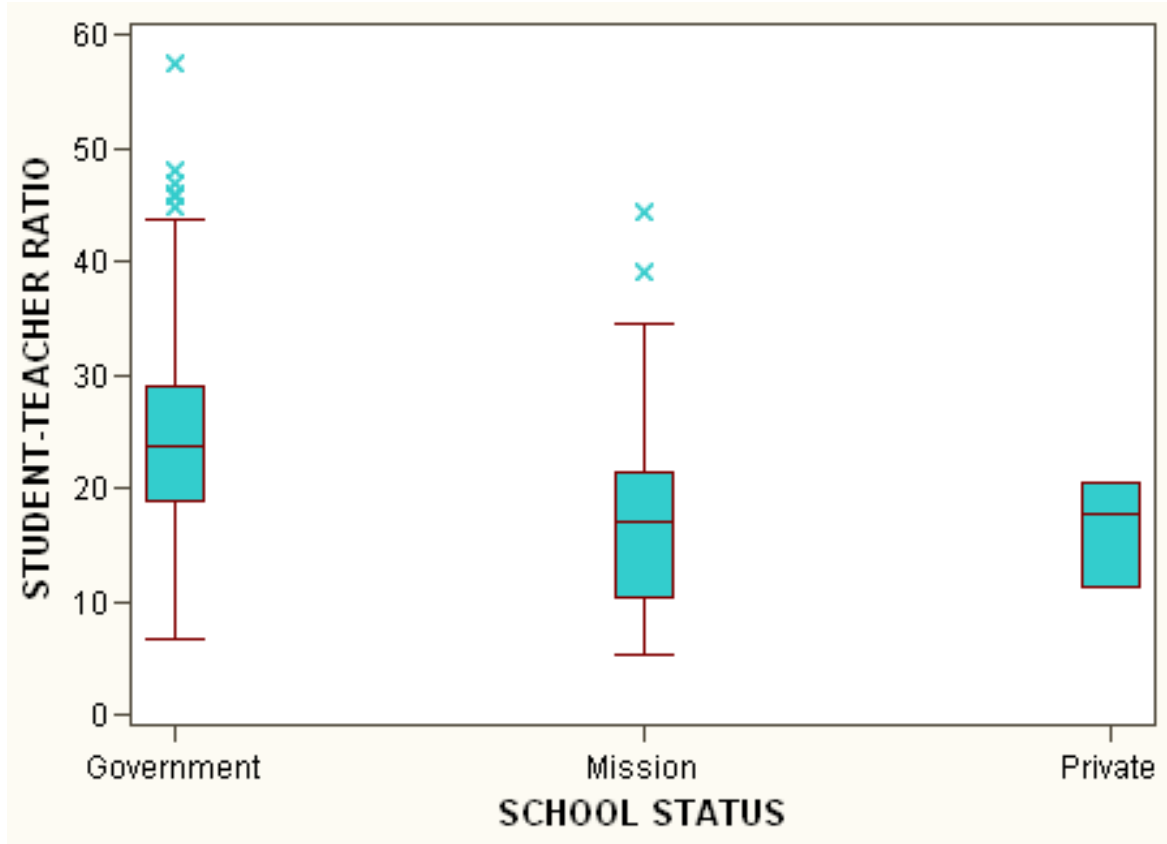


C. STR. SSG8: Distribution of the Student-Teacher Ratio by School Status (2004)

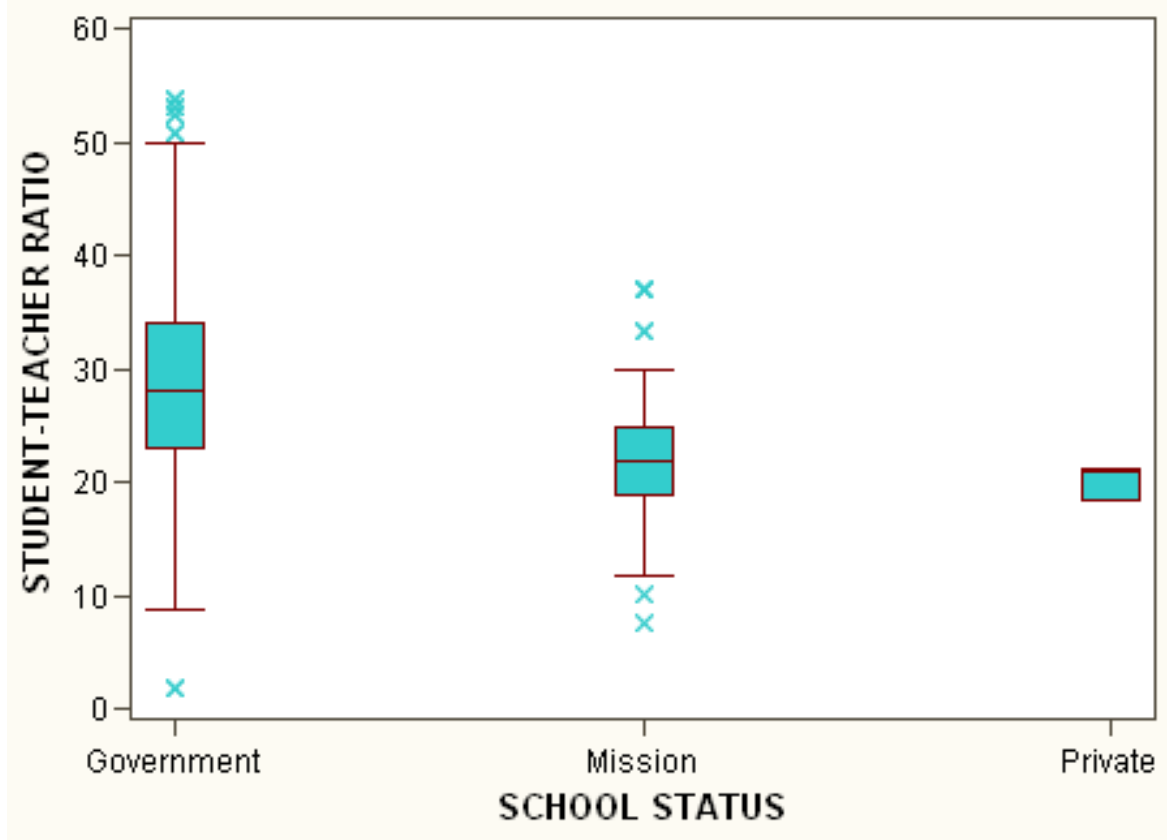

C. STR. SSG9: Distribution of the Student-Teacher Ratio by School Status (2006)

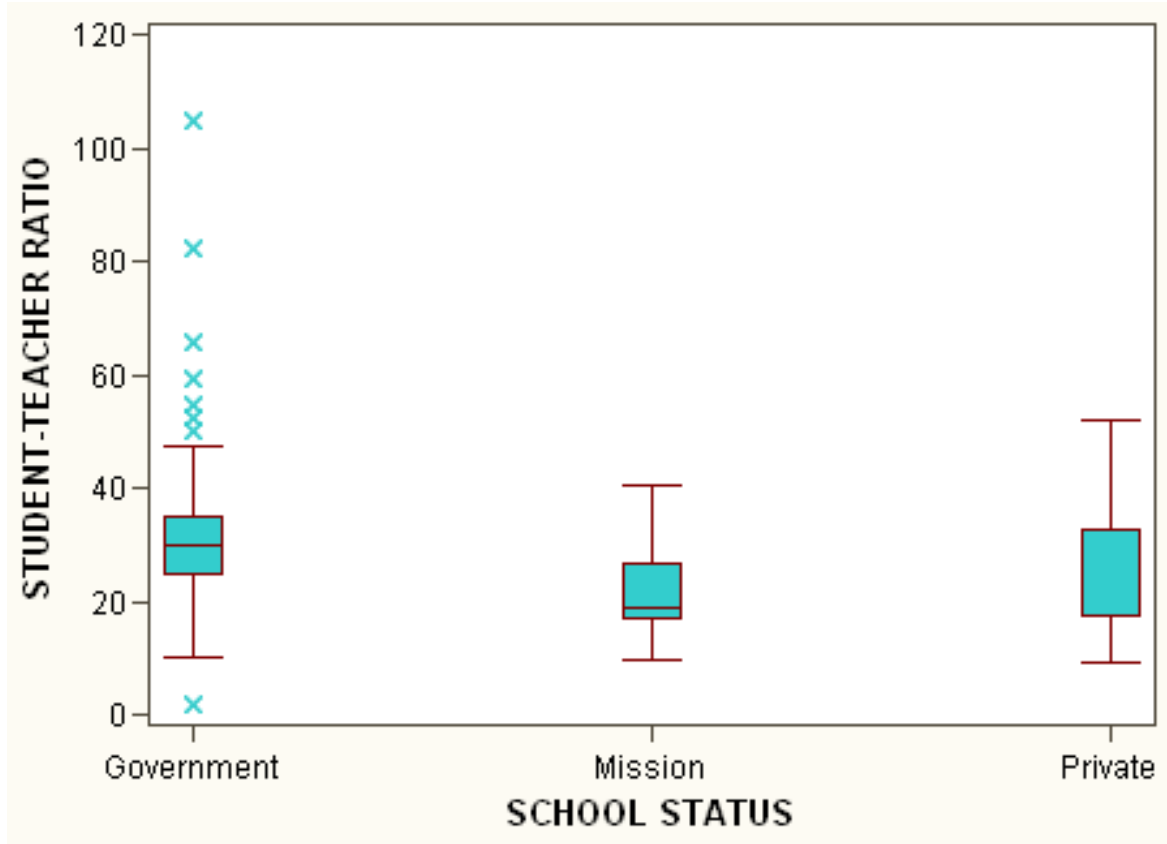


C. STR. SLG1: Distribution of the Student-Teacher Ratio by School Level (1995)

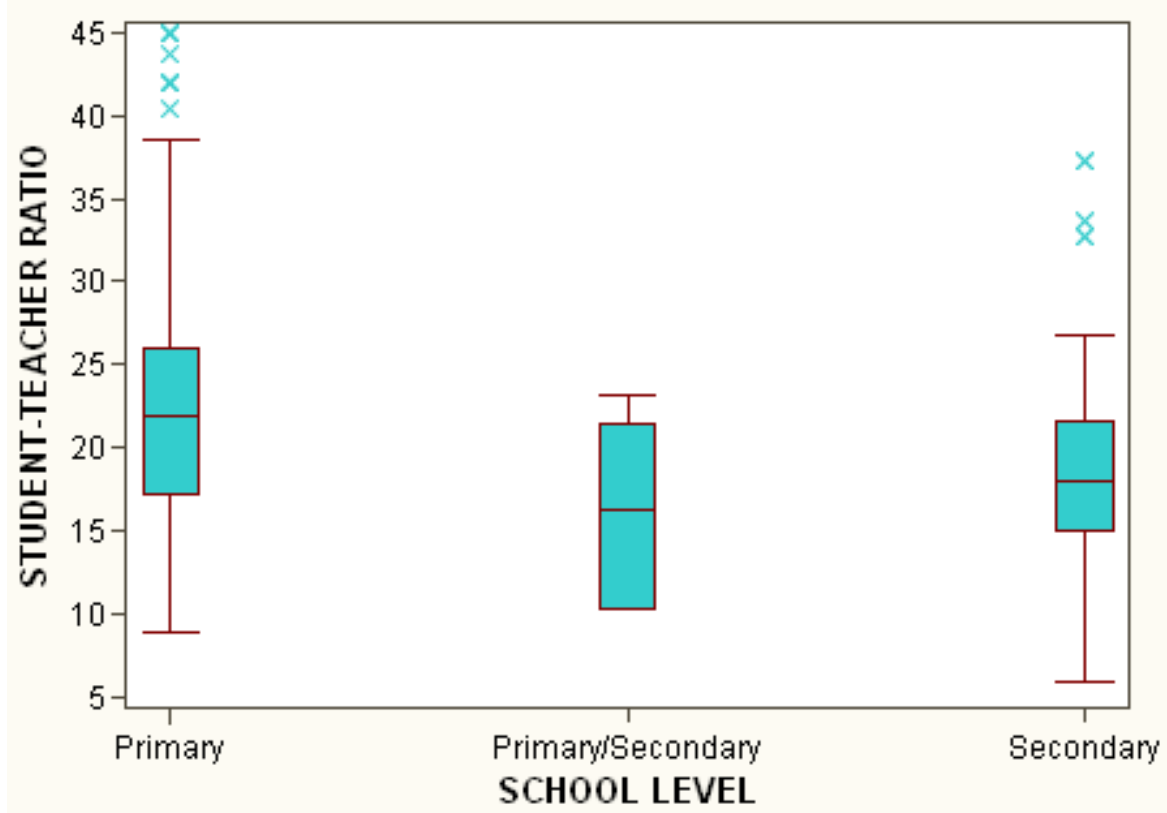

C. STR. SLG2: Distribution of the Student-Teacher Ratio by School Level (1996)

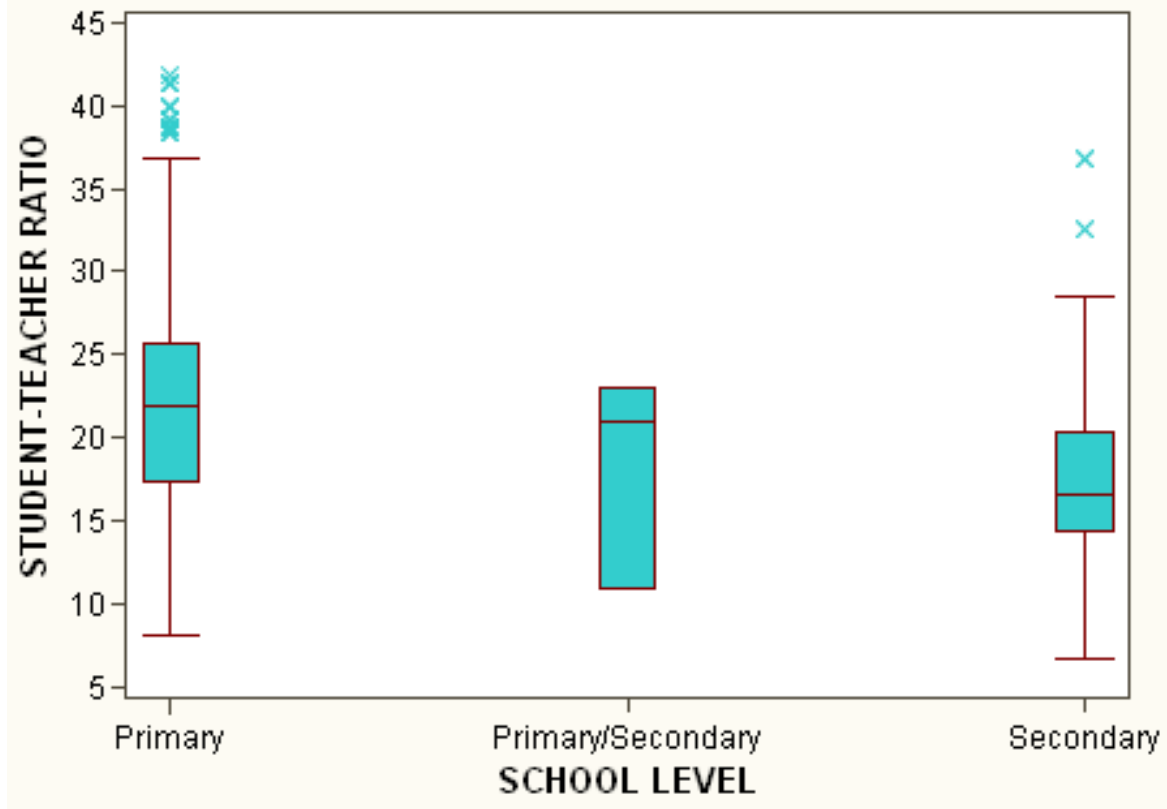


C. STR. SLG3: Distribution of the Student-Teacher Ratio by School Level (1997)

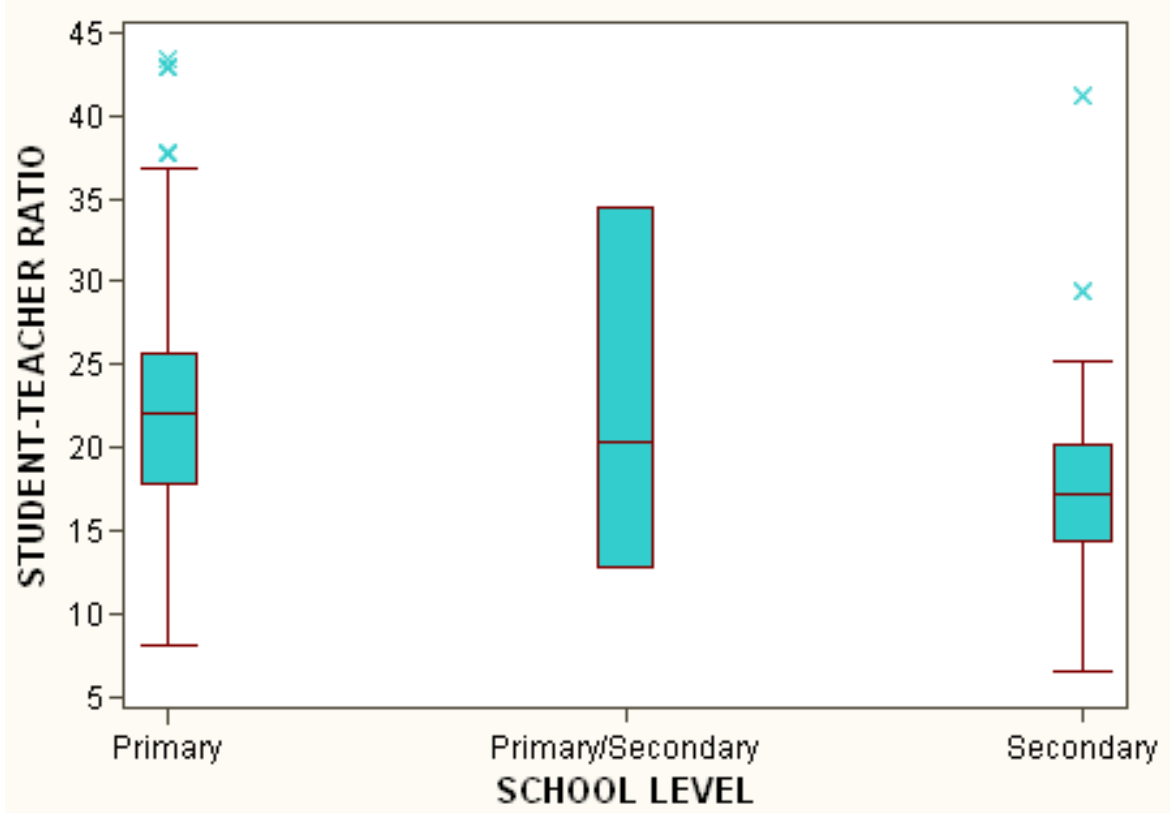

C. STR. SLG4: Distribution of the Student-Teacher Ratio by School Level (1998)

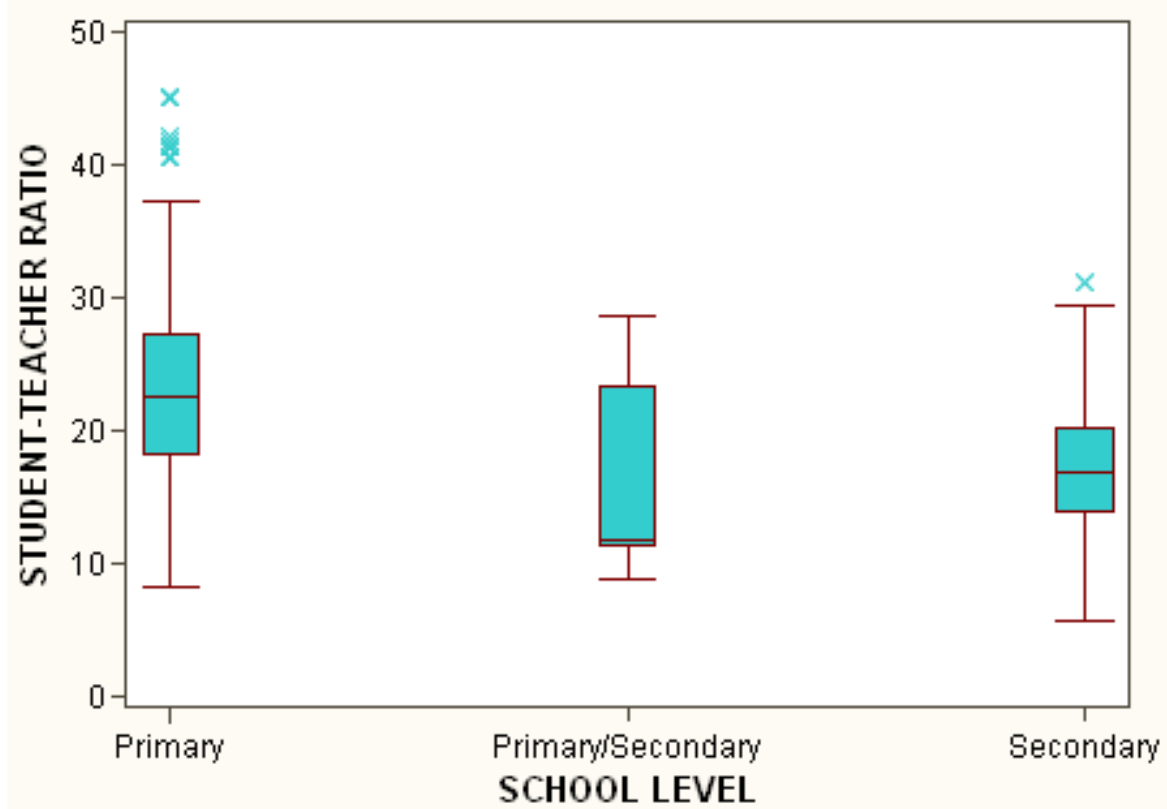


C. STR. SLG5: Distribution of the Student-Teacher Ratio by School Level (1999)

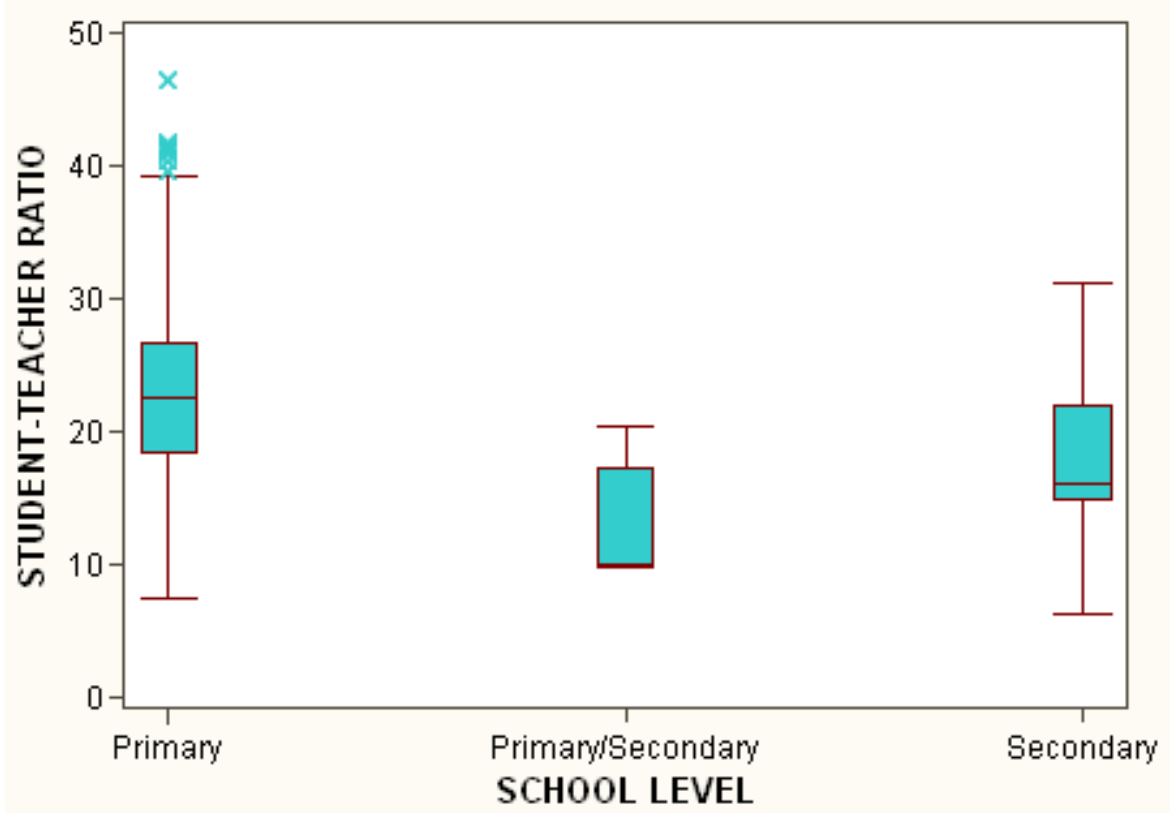

C. STR. SLG6: Distribution of the Student-Teacher Ratio by School Level (2000)

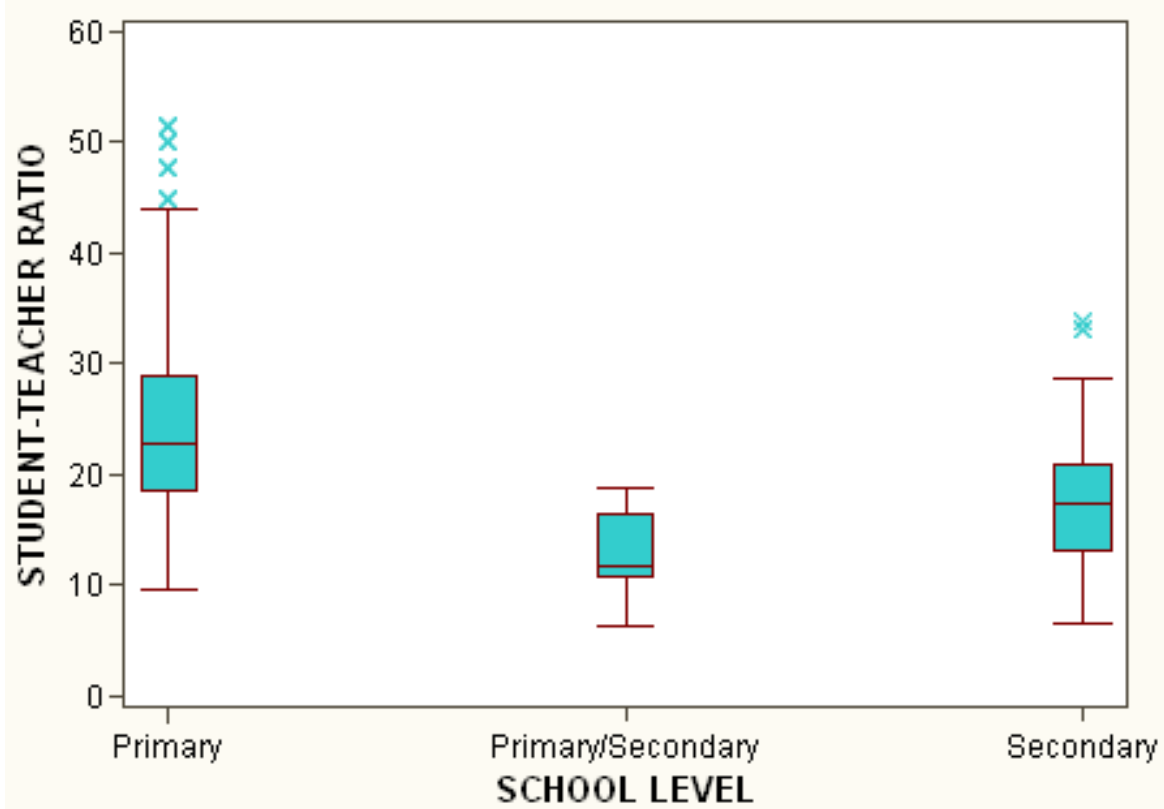


C. STR. SLG7: Distribution of the Student-Teacher Ratio by School Level (2001)

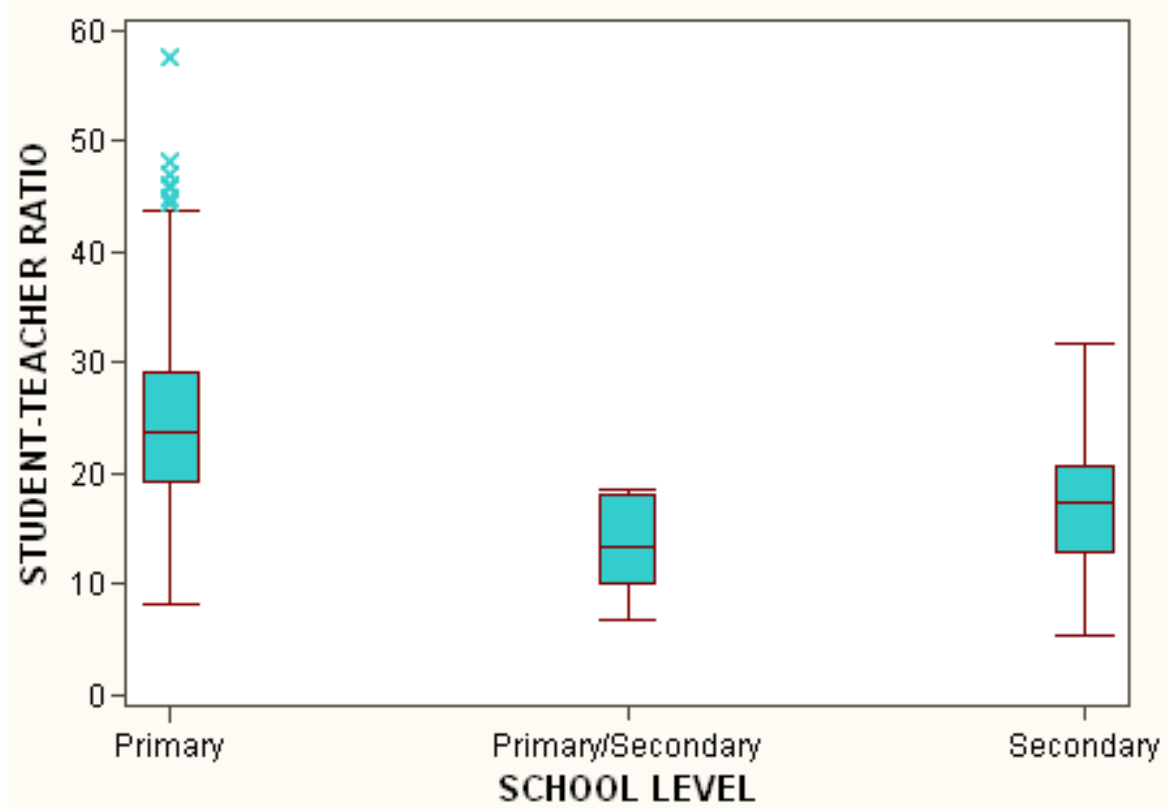


D. Copy of 2005 Census Form

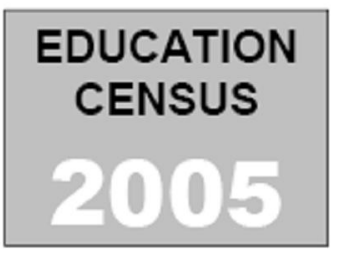

\section{Census Guide}

Samoa Ministry of Education, Sports \& Culture 


\section{Samoa}

\section{Education Census 2005}

Census Day is Wednesday, March $2^{\text {nd }} 2005$.

This is the official form for collecting data by the Samoa Ministry of Education. Census data are required by the Ministry for forward planning of education facilities, financial allocations and submissions and district and national enrolment projections. Information is also reported to Parliament and the UNESCO Institute for Statistics. The Ministry believes in promoting simple and effective access to public information and that appropriate information should be available as needed, whenever needed. At the same time it is recognised that the Ministry of Education, Sports \& Culture holds data about individuals and groups that are private and that this data needs to be treated with discretion.

This census includes all primary and secondary schools and colleges. Early childhood education, special education, vocational and tertiary education will be addressed by separate surveys.

The Ministry of Education, Sports \& Culture is striving towards achieving better opportunities for all learners in Samoa. We thank all schools and staff for your important participation in Census 2005

\section{Census Instructions}

1. Read the Census Guide.

2. Please use a blue or black pen. It is not necessary to type your responses on the form.

3. Section 1 and Section 6 are to be completed by all schools. Any information that is already available to the Ministry has been printed on the census form. If anything is incorrect, then please rule a line through it and write the correction beside it.

4. Sections 2 to 5 refer to primary and secondary level enrolments and attendance. Please answer the questions that apply to the levels taught at your school.

5. Principals are requested to complete the Principal's Checklist and return the completed census either through the Ministry's Mail System (government schools only) or to the Ministry's main office (nonGovernment Schools) by March $11^{\text {th }}, 2005$. The mailing address for census returns is as follows:

Policy, Planning and Research Division, Ministry of Education, Sports, \& Culture, P.O. Box 1869 , APIA.

Please refer to the Census Guide for more detailed instructions on completing the form. For additional information about this census or for help on completing the census form, please contact Lauiti'iti Ma'ia'i, PEO Information Analysis on 21911 (Ext. 724) or lauitiiti(c)lesamoa.net 


\section{Table of Contents}

Introduction .................................................................................

Census process in each school ...................................................... 1

Instructions for answering the census questions .......................... 2 


\section{Introduction}

It is that time of year again when the Samoa Ministry of Education, Sports \& Culture seeks the help of all govemment and non-govemment schools in conducting the annual Education Census. The census day will be

Wednesday, March 2nd 2005. It is anticipated that all schools will have completed and returned the census forms by $4.00 \mathrm{pm}$ to the Ministry of Education, Sports \& Culture on Friday, March $11^{\text {th }}$ 2005. A list of dates for the collection of the completed Census Forms from Government $\mathbf{S}$ chools is included as a separate sheet in the Census Package.

\section{Census process in each school}

The census return needs to be accurate and completed promptly. Therefore, the completion of the census within the school needs to be conducted in an organised and efficient manner. The Principal and the School Review Officer (for Govemment schools) will need to sign off the census forms as being accurate and completed before retuming them to the Ministry of Education, Sports \& Culture. This does not mean that the Principal has to do all of the work. Each school needs to establish an efficient process to allow the Principal to be confident in the results.

For small schools, it is recommended that Principals be responsible for completing the census and for ensuring that any forms distributed to teachers are completed accurately.

In larger schools the Principal may delegate census duties to the DeputyPrincipal (or equivalent) who with the assistance of the teachers will complete the census forms and retum it to the Principal for his/her approval. 


\section{Instructions for answering the census questions}

Questions 1 - 13.

Where the question contains information already, check that this is correct (including spelling). If it's not correct, draw a line through the information provided and write the corrected information neatly next to it.

Questions 14 -19. These refer to Primary and Secondary Teaching Staff. Please include the Principal even if he/she is not teaching. However, please do not include Pre-school teachers.

Question 14. Number of teaching staff?

Enter the number of teachers currently working at your school (only full-time teachers). Please include the Principal even if he/she is not teaching

Question 15. Number of female teachers?

Enter the number of female teaching staff. Include full-time teachers only.

Question 16. Number of Samoan teachers?

Enter the number of teachers who are Samoan citizens.

Question 17. Number of teachers who have completed teacher training? Enter the number of teachers who have completed training at a recognized teacher training institution e.g. NUS, Victoria University, USP, Western Samoa Teachers College, etc.

Question 18. Number of teachers with a degree?

Enter the number of teachers who have a degree e.g. B.A., B.Sc., B.Ed., M.A., Ph.D. etc.

Question 19. Number of teachers at each level?

Enter the number of teachers for each level. For those schools that provide more than one level e.g. primary and secondary, you should record separately the number of primary and secondary teachers but do not include a teacher in more than one level. Please note that the total for this question should be the same as question 14

Question 20. Total number of non-teaching staff?

Enter the number of non-teaching staff. Non-teaching staff includes all full-time and part-time administrative, maintenance, hostel staff etc.

Question 21. Primary level enrolments by age and sex as on Mareh 2nd 2005? This question is to be completed by all primary schools and all other schools that provide primary level classes. Include all students (even though they may have been absent on the day) who were still on the school roll on March 2nd 2005. Enter the number of students (including the number of repeaters) at each age and year level. Repeaters are any students repeating the year, whether they attended your school last year or not. 
Question 22. Secondary level enrolments by age and sex as on Mareh 2nd 2005?

This question is to be completed by all secondary schools and colleges and all other schools that provide secondary level classes. Include all students (even though they may have been absent on the day) who were still on the school roll on March 2nd 2005. Enter the number of students (including the number of repeaters) at each age and year level. Repeaters are any students repeating the year, whether they attended your school last year or not.

Question 23. Primary level enrolments and elasses on Mareh 2nd 2005? This question is to be completed by all schools that provide primary level classes. Identify every class that currently exists at the school (see example below). The table is structured to cater for single-grade and multi-grade classes. Include all students (even though they may have been absent on the day) who were still on the school roll on March 2nd 2005.

\begin{tabular}{|c|c|c|c|c|c|c|c|c|c|c|}
\hline \multirow{2}{*}{ Clas Name } & \multirow{2}{*}{ Teacher's Name } & \multicolumn{9}{|c|}{ Lexels 1augm in ints class } \\
\hline & & Yr1 & $Y+2$ & ri3 & Yr4 & Yr5 & Yro & $Y_{T} T$ & Yra & Tatal \\
\hline Yoan 15 & Alss Katatb & 30 & & & & & & & & 30 \\
\hline$Y_{\text {ear } 17} 17$ & Miss Tina & 29 & & & & & & & & 29 \\
\hline Yoan $2 / 3 \mathrm{~V}$ & Ais Vistar & & 55 & 70 & & & & & & 25 \\
\hline Year $4 / 65$ & Miss Sitith & & & & 12 & It & & & & 24 \\
\hline Year 6 it & Miss Moors & & & & & & 25 & & & 28 \\
\hline rear 7 ras & Mos theredith & & & & & & & 13 & 54 & 27 \\
\hline
\end{tabular}

Question 24. Secondary level enrolments and classes on Mareh 2nd 2005? This question is to be completed by all schools and colleges that provide secondary level classes. Include all subjects that are taught at your school. Please list under "other" any subjects that have not been listed. Include all students (even though they may have been absent on the day) who were still on the school roll on March 2nd 2005.

Question 25. Primary level students absent on Mareh 2nd 2005?

This question is to be completed by all schools that provide primary level classes. Only include students that were absent from school on March 2nd 2005.

Question 26. Secondary level students absent on March 2nd 2005? This question is to be completed by all schools that provide secondary leve classes. Only include students that were absent from school on March 2nd 2005.

Question 27. What type of buildings? Enter (or update) the table given. To determine the area refer to figure shown. Please enter a value for all items (enter a 0 against building types that your school does not have).

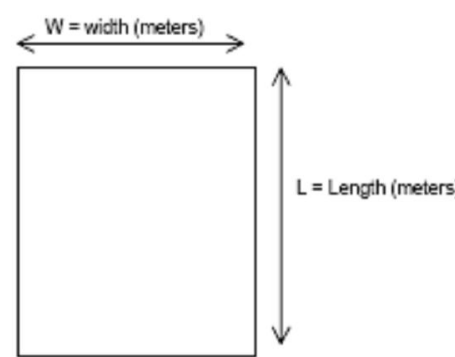

Area $($ sqm $m)=W \times L$ 
Question 28. Number of to ilets bowls?

Enter (or update) the number of toilets (toilet bowls) that are currently in use. Enter 0 if there are no tolets at your school.

Question 29. Number of urinals?

Enter (or update) the number of urinals that are currently in use. Enter 0 if there are no urinals at your school.

Question 30. Number of washbasins in the toilet block(s)?

Enter (or update) the number of washbasins located in the toilet block(s) that are currently in use. Enter 0 if there are no washbasins in the toilet blocks.

Question 31. Number of drinking fountains?

Enter (or update) the number of drinking fountains that are currently in use.

Only include drinking fountains. Do not include general-purpose taps. Enter 0 if there are no drinking fountains.

Question 32. What type of water supply?

Tick the option that applies to your school. Draw a line through any option that is incorrectly ticked.

Question 33. What type of eleetrieity supply?

Tick the option that applies to your school. Draw a line through any option that is incorrectly ticked.

Question 34. Is there a telephone line inte the sehool?

Tick "Yes" if there is a telephone line that is working.

Question 35. What type of elassroom furniture is cURRENTLY in use? Enter (or update) all items in the table given. Do not include fumiture that is not being used (in need of repair). Enter a 0 for furniture types that your school does not have.

Question 36. What type of staffroom furniture is CURRENTLY in use? Enter (or update) all items in the table given. Do not include fumiture that is not being used (in need of repair). Enter a 0 for furniture types that your school does not have.

Question 37. What equipment furniture is CURRENTLY in use?

Enter (or update) all items in the table given. Enter a 0 against items of equipment that the school does not have. Do not include equipment that is permanently out of service (i.e. cannot be repaired) 


\section{EDUCATION \\ CENSUS \\ Principal's Checklist}

This Census Checklist is to be completed by Principals of both Government and non-Government schools.

\section{Before the census}

a Familiarise yourself with the Census form

a Read the Census instructions (first page of the Census Form) and Census Guide.

a Identify staff responsible for collecting the census data and completing the forms.

\section{After the census forms have been completed}

This part of the checklist is to be completed by all Principals before returning the Census Form. For Government schools, the checklist is to be completed jointly by the Principal and the School Review Officer.

Please check each answer in the census and enter the following in the "Completed" column in the table below.

Yes If the question has been completed correctly. You do not need to write anything under the "Comments" column if you answer Yes.

N/A Not applicable e.g. Question 21 (Primary level enrolments) will only apply to schools that provide primary level classes. This question will not be applicable (N/A) to secondary schools and colleges.

No If the question is applicable but could not be answered then enter the reason in the "Comments" column.

Please ensure that all questions in Section 6 (Facilities and Assets) have a response, even if it's " 0 ".

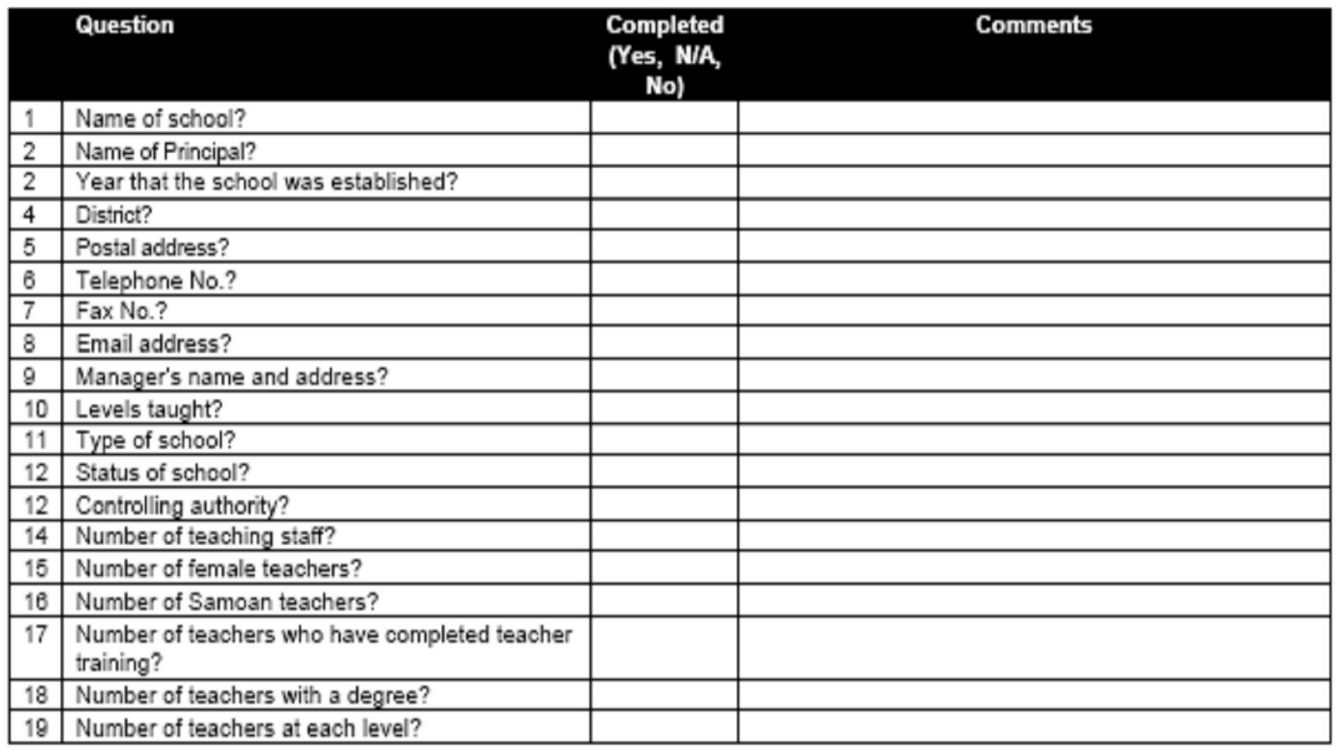




\begin{tabular}{|c|c|c|c|}
\hline & Question & $\begin{array}{c}\text { Completed } \\
\text { (Yes, N/A, } \\
\text { No) }\end{array}$ & Comments \\
\hline 20 & Total number of non-teaching staff? & & \\
\hline 21 & $\begin{array}{l}\text { Primary level enrolments by age and sex as on } \\
\text { March } 2,2005 \text { ? }\end{array}$ & & \\
\hline 22 & $\begin{array}{l}\text { Secondary level enrolments by age and sex as } \\
\text { on March } 2,2005 \text { ? }\end{array}$ & & \\
\hline 22 & $\begin{array}{l}\text { Primary lewel enrolments and classes on March } \\
2,2005 \text { ? }\end{array}$ & & \\
\hline 24 & $\begin{array}{l}\text { Secondary level enrolments and classes on } \\
\text { March } 2,2005 \text { ? }\end{array}$ & & \\
\hline 25 & Primary lewel students absent on March 2, 2005? & & \\
\hline 26 & $\begin{array}{l}\text { Secondary level students absent on March } 2, \\
2005 ?\end{array}$ & & \\
\hline 27 & What type of buildings? & & \\
\hline 28 & Number of toilet bowls? & & \\
\hline 29 & Number of urinals? & & \\
\hline 20 & Number of washbasins in the toilet blockis)? & & \\
\hline 21 & Number of drinking fountains? & & \\
\hline 22 & What types of water supply? & & \\
\hline 22 & What types of electricity supply? & & \\
\hline 24 & Is there a telephone line into the school? & & \\
\hline$\frac{-7}{25}$ & $\begin{array}{l}\text { What type of classroom furniture is CURRENTLY } \\
\text { in use? }\end{array}$ & & \\
\hline 26 & $\begin{array}{l}\text { What type of staffroom furniture is CURRENTLY } \\
\text { in use? }\end{array}$ & & \\
\hline 27 & What equipment is CURRENTLY in use? & & \\
\hline
\end{tabular}

a The following is to be returned to the Ministry of Education, Sports \& Culture's office at Malifa by $4: 00 \mathrm{p} . \mathrm{m}$., March $11^{\text {th }} 2005$ along with your school's Census Form.

a For Government Schools - Only schools whose mail is picked up on Friday, $11^{\text {th }}$ March 2005 is required to have all their census forms returned to the Ministry on that day by 4:00 p.m. Those schools whose mail is picked up on Monday, Tuesday, Wednesday and Thursdays are required to have their census forms in by 4:00 p.m. of the same days in the following week starting Monday, $14^{\text {th }}$ March 2005 to Thursday, 17 $7^{\text {th }}$ March 2005. Census forms received after the days identified for collection will be noted as late.

a The Census form.

๑ This Principal's Checklist.

The Ministry of Education, Sports \& Culture mailing address for this census is as follows:

Policy, Planning and Research Division

Ministry of Education, Sports \& Culture,

P. O. Box 1869

APIA

Principal's signature

Date

School Review Officer's signature

Date 


\section{Section 1. General Information}

1 Name of school? Aana No.1 Secondary School

2 Name of Principal?

Iemasae Viliamu Tilialo

3 Year that the school was established? 1956

4 District?

Aana No. 1

5 Postal address?

P.O.Box 1696, Apia, Western Samoa

P.O.Box 1696, Apla, Western Samoa

\begin{tabular}{lll|}
6 & Telephone No.? \\
$\mathbf{7}$ & Fax No.? \\
8 & Email address? \\
None \\
$\mathbf{9}$ Manager's name and address? \\
Tupae Esera \\
\hline Department of Education
\end{tabular}

10 Levels taught? (Tick any that apply.)

Primary

$\checkmark$ Secondary

11 Type of school? (Tick one only.

$\checkmark$ Co-education

Boys only

Girls only

12 Status of school? (Tick one only.)

$\checkmark$ Government

Mission

Private
13 Controlling authority?

Tick one only, or complete the "Other" box.)

$\checkmark$ Government

Private

Catholic

Methodist

C.C.C.S.

S.D.A.

L.D.S

Peace Chapel

Baptist

Other

PRIMARY and SECONDARY TEACHING STAFF

14 Number of teaching staff?

(Include Principals that are not teaching.)

15 Number of female teachers?

16 Number of Samoan teachers?

17 Number of teachers who have completed teacher training? (i.e. From a recognised teacher training institution.)

18 Number of teachers with a degree? (e.g. B.Sc., B.Ed., M.A., Ph.D. etc.)
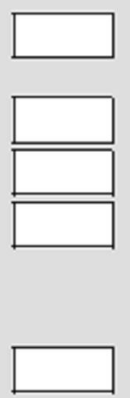

19 Number of teachers at each level? (Do not include a teacher in more than one level. The total of all levels must equal the total in

Primary

Secondary

Special Education

TOTAL

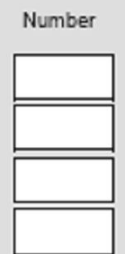

NON-TEACHING STAFF

20 Total number of non-teaching staff?

(Include all full-time and part-time, non-teaching staff.)

\begin{tabular}{|l|l|l|}
\hline \multicolumn{3}{|c|}{ OFFICE USE ONLY } \\
\hline \multicolumn{3}{|c|}{ 8 Aana No.1 Secondary School } \\
\hline Entered by and Date & & \\
\hline Checked by and Date & & \\
\hline
\end{tabular}

Education Census 2005 Form Version 2

8 Aana No.1 Secondary Schoo 


\section{Section 2. Enrolment and Age}

21 Primary level enrolments by age and sex as on Census day. (Enter the number of students (including repeaters) at each age and year level. Also enter the number of repeaters in the last column. Repeaters are ANY students repeating the year, whether they attended your school last year or not)

\begin{tabular}{|c|c|c|c|c|c|c|c|c|c|c|c|c|}
\hline \multirow[b]{2}{*}{ Level } & \multirow[b]{2}{*}{ Sex } & \multicolumn{11}{|c|}{ Number of Students (including repeaters) } \\
\hline & & Age 5 & Age 6 & Age 7 & Age 8 & Age 9 & Age 10 & Age 11 & Age 12 & Age 13 & Age 14+ & Total \\
\hline \multirow{2}{*}{ Year 1} & M & & & & & & & & & & & \\
\hline & $\mathrm{F}$ & & & & & & & & & & & \\
\hline \multirow{2}{*}{ Year 2} & M & & & & & & & & & & & \\
\hline & $\mathrm{F}$ & & & & & & & & & & & \\
\hline \multirow{2}{*}{ Year 3} & M & & & & & & & & & & & \\
\hline & $\mathrm{F}$ & & & & & & & & & & & \\
\hline \multirow{2}{*}{ Year 4} & M & & & & & & & & & & & \\
\hline & $\mathrm{F}$ & & & & & & & & & & & \\
\hline \multirow{2}{*}{ Year 5} & M & & & & & & & & & & & \\
\hline & $\mathrm{F}$ & & & & & & & & & & & \\
\hline \multirow{2}{*}{ Year 6} & M & & & & & & & & & & & \\
\hline & $\mathrm{F}$ & & & & & & & & & & & \\
\hline \multirow{2}{*}{ Year 7} & M & & & & & & & & & & & \\
\hline & $\mathrm{F}$ & & & & & & & & & & & \\
\hline \multirow{2}{*}{ Year 8} & M & & & & & & & & & & & \\
\hline & $\mathrm{F}$ & & & & & & & & & & & \\
\hline
\end{tabular}

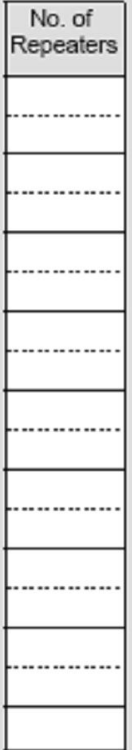

22 Secondary level enrolments by age and sex as on Census day.

(Enter the number of students (induding repeaters) at each age and year level. Also enter the number of repeaters in the last column. Repeaters are ANY students repeating the year, whether they attended your school last year or not.)

\begin{tabular}{|c|c|c|c|c|c|c|c|c|c|c|c|}
\hline \multirow{2}{*}{ Level } & \multirow[b]{2}{*}{ Sex } & \multicolumn{10}{|c|}{ Number of Students (including repeaters) } \\
\hline & & Age 11 & Age 12 & Age 13 & Age 14 & Age 15 & Age 16 & Age 17 & Age18 & Age 19 Age $20+$ & Total \\
\hline \multirow[t]{2}{*}{ Year 9} & M & & & & & & & & & & \\
\hline & $\mathrm{F}$ & & & & & & & & & & \\
\hline \multirow[t]{2}{*}{ Year 10} & $\mathrm{M}$ & & & & & & & & & & \\
\hline & F & & & & & & & & & & \\
\hline \multirow[t]{2}{*}{ Year 11} & $\mathrm{M}$ & & & & & & & & & & \\
\hline & $\mathrm{F}$ & & & & & & & & & & \\
\hline \multirow[t]{2}{*}{ Year 12} & M & & & & & & & & & & \\
\hline & $\mathrm{F}$ & & & & & & & & & & \\
\hline \multirow[t]{2}{*}{ Year 13} & M & & & & & & & & & & \\
\hline & $\mathrm{F}$ & & & & & & & & & & \\
\hline
\end{tabular}

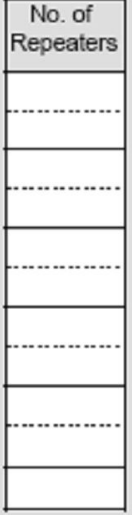




\section{Section 3. Primary Level Enrolment and Classes}

23 Primary level enrolments and classes as on Census day. (To be completed by all schools that provide PRIMARY level classes. Please refer to the Census Guide for instructions).

\begin{tabular}{|c|c|c|c|c|c|c|c|c|c|c|}
\hline \multirow{2}{*}{ Class Name } & \multirow{2}{*}{ Teacher's Name } & \multicolumn{9}{|c|}{ Number of Students at Each Level } \\
\hline & & Yr 1 & Yr2 & Yr3 & Yr 4 & Yr 5 & Yr 6 & Yr 7 & Yr 8 & Total \\
\hline & & & & & & & & & & \\
\hline & & & & & & & & & & \\
\hline & & & & & & & & & & \\
\hline & & & & & & & & & & \\
\hline & & & & & & & & & & \\
\hline & & & & & & & & & & \\
\hline & & & & & & & & & & \\
\hline & & & & & & & & & & \\
\hline & & & & & & & & & & \\
\hline & & & & & & & & & & \\
\hline & & & & & & & & & & \\
\hline & & & & & & & & & & \\
\hline & & & & & & & & & & \\
\hline & & & & & & & & & & \\
\hline & & & & & & & & & & \\
\hline & & & & & & & & & & \\
\hline & & & & & & & & & & \\
\hline & & & & & & & & & & \\
\hline & & & & & & & & & & \\
\hline & & & & & & & & & & \\
\hline & & & & & & & & & & \\
\hline & & & & & & & & & & \\
\hline & & & & & & & & & & \\
\hline & & & & & & & & & & \\
\hline & & & & & & & & & & \\
\hline & & & & & & & & & & \\
\hline & & & & & & & & & & \\
\hline & & & & & & & & & & \\
\hline & & & & & & & & & & \\
\hline & & & & & & & & & & \\
\hline & & & & & & & & & & \\
\hline & & & & & & & & & & \\
\hline & & & & & & & & & & \\
\hline & & & & & & & & & & \\
\hline & & & & & & & & & & \\
\hline & & & & & & & & & & \\
\hline & & & & & & & & & & \\
\hline & & & & & & & & & & \\
\hline & & & & & & & & & & \\
\hline & & & & & & & & & & \\
\hline & & & & & & & & & & \\
\hline
\end{tabular}




\section{Section 4. Secondary Level Enrolment and Classes}

24 Secondary level enrolments and classes as on Census day.

(To be completed by all schools and colleges that provide SECONDARY level classes. Note: Enter the number of classes, not periods).

\begin{tabular}{|c|c|c|c|c|c|}
\hline \multirow{2}{*}{$\begin{array}{l}\text { Level } \\
\text { (Year) }\end{array}$} & \multirow{2}{*}{ Subject } & \multicolumn{2}{|c|}{ No. of Students } & \multirow{2}{*}{$\begin{array}{l}\text { No. of } \\
\text { Teachers }\end{array}$} & \multirow{2}{*}{$\begin{array}{l}\text { No. of } \\
\text { Classes }\end{array}$} \\
\hline & & Males & Females & & \\
\hline 9 & Samoan & & & & \\
\hline 9 & English & & & & \\
\hline 9 & Mathematics & & & & \\
\hline 9 & Social Studies & & & & \\
\hline 9 & Science & & & & \\
\hline 9 & Business Studies & & & & \\
\hline 9 & Agricultural Science & & & & \\
\hline \multirow[t]{2}{*}{9} & Design and Technology (Ind. Arts) & & & & \\
\hline & Other Subjects & & & & \\
\hline 9 & Physical Education & & & & \\
\hline 9 & Music & & & & \\
\hline 9 & Religious Studies & & & & \\
\hline 9 & Typing/Shorthand & & & & \\
\hline 9 & Fine Arts & & & & \\
\hline 9 & Office Management & & & & \\
\hline 9 & Other (please specify) & & & & \\
\hline 9 & Other (please specify) & & & & \\
\hline & & & & & \\
\hline & & & & & \\
\hline & & & & & \\
\hline 10 & Samoan & & & & \\
\hline 10 & English & & & & \\
\hline 10 & Mathematics & & & & \\
\hline 10 & Social Studies & & & & \\
\hline 10 & Science & & & & \\
\hline 10 & Business Studies & & & & \\
\hline 10 & Agricultural Science & & & & \\
\hline \multirow[t]{2}{*}{10} & Design and Technology (Ind. Arts) & & & & \\
\hline & Other Subjects & & & & \\
\hline 10 & Physical Education & & & & \\
\hline 10 & Music & & & & \\
\hline 10 & Religious Studies & & & & \\
\hline 10 & Typing/Shorthand & & & & \\
\hline 10 & Fine Arts & & & & \\
\hline 10 & Office Management & & & & \\
\hline 10 & Other (please specify) & & & & \\
\hline 10 & Other (please specify) & & & & \\
\hline & & & & & \\
\hline & & & & & \\
\hline & & & & & \\
\hline & & & & & \\
\hline
\end{tabular}




\section{Section 4. (continued)}

24 Continued.

\begin{tabular}{|c|c|c|c|c|c|}
\hline \multirow{2}{*}{$\begin{array}{l}\text { Level } \\
\text { (Year) }\end{array}$} & \multirow{2}{*}{ Subject } & \multicolumn{2}{|c|}{ No. of Students } & \multirow{2}{*}{$\begin{array}{l}\text { No. of } \\
\text { Teachers }\end{array}$} & \multirow{2}{*}{$\begin{array}{l}\text { No. of } \\
\text { Classes }\end{array}$} \\
\hline & & Males & Females & & \\
\hline 11 & Samoan & & & & \\
\hline 11 & English & & & & \\
\hline 11 & Mathematics & & & & \\
\hline 11 & Social Studies & & & & \\
\hline 11 & Science & & & & \\
\hline 11 & Business Studies & & & & \\
\hline 11 & Agricultural Science & & & & \\
\hline \multirow[t]{2}{*}{11} & Design and Technology (Ind. Arts) & & & & \\
\hline & Other Subjects & & & & \\
\hline 11 & Physical Education & & & & \\
\hline 11 & Religious Studies & & & & \\
\hline 11 & Music & & & & \\
\hline 11 & Typing/Shorthand & & & & \\
\hline 11 & Fine Arts & & & & \\
\hline 11 & Office Management & & & & \\
\hline 11 & Other (please specify) & & & & \\
\hline 11 & Other (please specify) & & & & \\
\hline 12 & Samoan & & & & \\
\hline 12 & English & & & & \\
\hline 12 & Mathematics & & & & \\
\hline 12 & Geography & & & & \\
\hline 12 & History & & & & \\
\hline 12 & Accounting & & & & \\
\hline 12 & Economics & & & & \\
\hline 12 & Biology & & & & \\
\hline 12 & Chemistry & & & & \\
\hline 12 & Physics & & & & \\
\hline 12 & Science & & & & \\
\hline 12 & Agricultural Science & & & & \\
\hline 12 & Design and Technology (Ind. Arts) & & & & \\
\hline 12 & Food and Textile Technology (Home Econ.) & & & & \\
\hline 12 & Computer Studies & & & & \\
\hline \multirow[t]{2}{*}{12} & Developmental Studies & & & & \\
\hline & Other Subjects & & & & \\
\hline 12 & Physical Education & & & & \\
\hline 12 & Music & & & & \\
\hline 12 & Religious Studies & & & & \\
\hline 12 & Typing/Shorthand & & & & \\
\hline 12 & Fine Arts & & & & \\
\hline 12 & Office Management & & & & \\
\hline 12 & Other (please specify) & & & & \\
\hline 12 & Other (please specify) & & & & \\
\hline
\end{tabular}


Section 4. (continued)

24 Continued.

\begin{tabular}{|c|c|c|c|c|c|}
\hline \multirow{2}{*}{$\begin{array}{l}\text { Level } \\
\text { (Year) }\end{array}$} & \multirow{2}{*}{ Subject } & \multicolumn{2}{|c|}{ No. of Students } & \multirow{2}{*}{$\begin{array}{l}\text { No. of } \\
\text { Teachers }\end{array}$} & \multirow{2}{*}{$\begin{array}{l}\text { No. of } \\
\text { Classes }\end{array}$} \\
\hline & & Males & Females & & \\
\hline 13 & Samoan & & & & \\
\hline 13 & English & & & & \\
\hline 13 & Mathematics & & & & \\
\hline 13 & Gecgraphy & & & & \\
\hline 13 & History & & & & \\
\hline 13 & Accounting & & & & \\
\hline 13 & Economics & & & & \\
\hline 13 & Biology & & & & \\
\hline 13 & Chemistry & & & & \\
\hline 13 & Physics & & & & \\
\hline 13 & Science & & & & \\
\hline 13 & Agricultural Science & & & & \\
\hline 13 & Design and Technology (Ind. Arts) & & & & \\
\hline 13 & Food and Textile Technology (Home Econ.) & & & & \\
\hline 13 & Computer Studies & & & & \\
\hline \multirow[t]{2}{*}{13} & Developmental Studies & & & & \\
\hline & Other Subjects & & & & \\
\hline 13 & Physical Education & & & & \\
\hline 13 & Music & & & & \\
\hline 13 & Religious Studies & & & & \\
\hline 13 & Typing/Shorthand & & & & \\
\hline 13 & Fine Arts & & & & \\
\hline 13 & Office Management & & & & \\
\hline 13 & Other (please specify) & & & & \\
\hline 13 & Other (please specify) & & & & \\
\hline & & & & & \\
\hline & & & & & \\
\hline & & & & & \\
\hline & & & & & \\
\hline & & & & & \\
\hline & & & & & \\
\hline & & & & & \\
\hline & & & & & \\
\hline & & & & & \\
\hline & & & & & \\
\hline & & & & & \\
\hline & & & & & \\
\hline & & & & & \\
\hline & & & & & \\
\hline & & & & & \\
\hline & & & & & \\
\hline
\end{tabular}




\section{Section 5. Attendance}

25 Primary level students absent on Census day. (Enter the number of primary students that were absent on Census day.)

\begin{tabular}{|l|l|l|l|}
\hline \multirow{2}{*}{ Level } & \multicolumn{2}{|c|}{ Students Absent } & \multirow{2}{*}{ Total } \\
\cline { 2 - 3 } & Males & Females & \\
\hline Year 1 & & & \\
\hline Year 2 & & & \\
\hline Year 3 & & & \\
\hline Year 4 & & & \\
\hline Year 5 & & & \\
\hline Year 6 & & & \\
\hline Year 7 & & & \\
\hline Year 8 & & & \\
\hline
\end{tabular}

26 Secondary level students absent on Census day. (Enter the number of secondary students that were absent on Census day.)

\begin{tabular}{|c|c|c|c|}
\hline \multirow{2}{*}{ Level } & \multicolumn{2}{|c|}{ Students Absent } & \multirow{2}{*}{ Total } \\
\cline { 2 - 3 } & Males & Females & \\
\hline Year 9 & & & \\
\hline Year 10 & & & \\
\hline Year 11 & & & \\
\hline Year 12 & & & \\
\hline Year 13 & & & \\
\hline
\end{tabular}




\section{Section 6. Facilities and Assets}

27 What types of buildings?

\begin{tabular}{|lcc}
\hline \multicolumn{1}{|c}{ Type } & Total number & $\begin{array}{c}\text { Total floor area } \\
\text { (sq.m) }\end{array}$ \\
\hline Classroom & 10 & 640 \\
\hline Office & 1 & 16 \\
\hline Storeroom & 1 & 16 \\
\hline Workshop(Ind.Arts) & 1 & 64 \\
\hline Home/Ec. & 1 & 64 \\
\hline Art/Music & 0 & 0 \\
\hline Science Lab & 1 & 64 \\
\hline Canteen & 0 & 0 \\
\hline Hostel & 0 & 0 \\
\hline Hall & 1 & 128 \\
\hline Staffroom & 1 & 32 \\
\hline Library & 1 & 0 \\
\hline Samoan Fale & 1 & 0 \\
\hline Computer Lab & 0 & 0 \\
\hline
\end{tabular}

28 Number of toilet bowls?

29 Number of urinals?

30 Number of washbasins in the toilet block(s)?

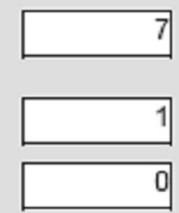

31 Number of drinking fountains?

0

32 What type of water supply?

SWA supply

$\checkmark$ Local supply (e.g.well, dam, tank, spring etc.)

None

33 What type of electricity supply?

$\checkmark$ EPC supply

School generator

None

34 Is there a telephone line into the school?

$\checkmark$ Yes

No
35 What classroom furniture is CURRENTLY in use?

(NB.Please count a two-seater bench as 2 seats and a twoseater desk as 2 desks. Refer to the Census Guide for more information.)

\begin{tabular}{|lc|}
\hline \multicolumn{1}{|c|}{ Type } & Number \\
\hline Classroom seats for students & 125 \\
\hline Classroom desks for students & 137 \\
\hline Classroom desks for teachers & 11 \\
\hline Classroom chairs for teachers & 8 \\
\hline Classroom blackboards & 28 \\
\hline
\end{tabular}

36 What staffroom furniture is CURRENTLY in use?

\begin{tabular}{|lc|}
\hline \multicolumn{1}{|c|}{ Type } & Number \\
\hline Staffroom chairs & 4 \\
\hline Staffroom tables & 1 \\
\hline Staffroom cupboards & 0 \\
\hline Staffroom pin boards & 2 \\
\hline Staffroom blackboards & 2 \\
\hline Staffroom sinks with taps & 0 \\
\hline
\end{tabular}

37 What equipment is CURRENTLY in use?

\begin{tabular}{|ll|}
\hline \multicolumn{1}{|c|}{ Type } & Number \\
\hline Bus & 0 \\
\hline Truck & 0 \\
\hline Other vehicle & 0 \\
\hline Duplicator & 0 \\
\hline Photocopier & 0 \\
\hline Typewriter & 0 \\
\hline Computer & 2 \\
\hline Radio & 0 \\
\hline Television/video & 0 \\
\hline Overhead projector & 0 \\
\hline First Aid cupboard/kit & 1 \\
\hline Fire extinguisher & 0 \\
\hline Telephone & 1 \\
\hline Fax & 0 \\
\hline Internet connection & 0 \\
\hline
\end{tabular}




\section{E. SAS Codes For Models}

\section{MODEL CODES:}

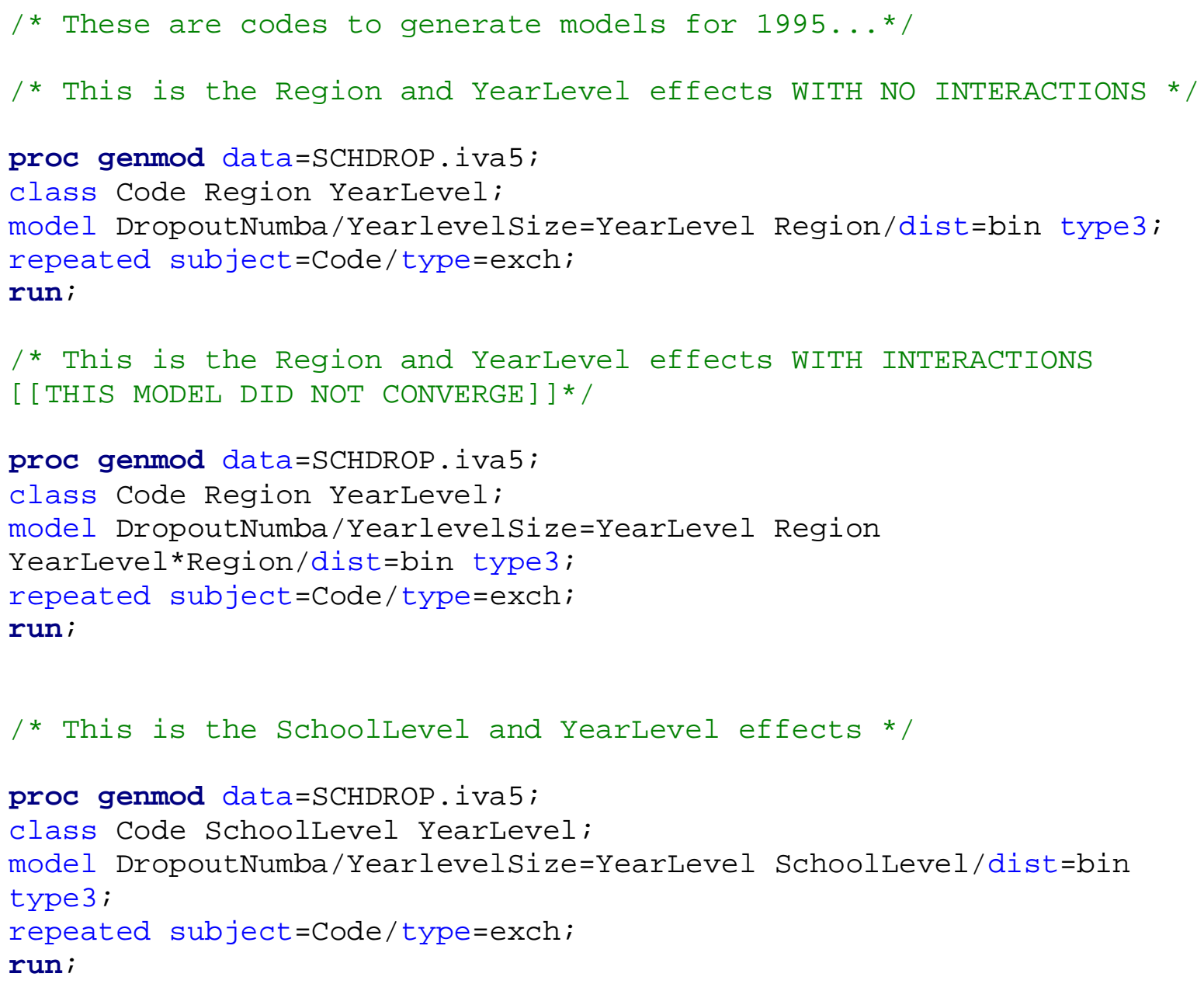


run;

/* This is the Schoolstatus and YearLevel effects WITH INTERACTIONS [THIS MODEL DID NOT CONVERGE SO USE ORDINARY LOGISTIC REGRESSION]*/

proc genmod data $=$ SCHDROP.iva5;

class Code Schoolstatus YearLevel;

model DropoutNumba/YearlevelSize=YearLevel Schoolstatus

YearLevel*Schoolstatus/dist=bin type3;

run;

/** This is the Schoolsize (TotEnrol), StudentTeachRatio, Region, Schoolstatus and Schoollevel effetcs

using the new dataset (ivaffou). SCHOOLSTATUS IS NOT SIGNIFICANT

(Drop) $* * /$

proc genmod data $=$ SCHDROP.iva 5 fou;

class Code Region Schoolstatus SchoolLevel;

model DropoutNumba/YearlevelSize=TotEnrol StudentTeachRatio Region

Schoolstatus SchoolLevel/dist=bin type3;

repeated subject $=$ Code $/$ ype $=$ exch;

run;

/** This is the StudentTeachRatio, Region, Schoolstatus and

Schoollevel effetcs

using the new dataset (ivaffou). SCHOOLSTATUS IS NOT SIGNIFICANT

(Drop) $\star \star /$

proc genmod data $=$ SCHDROP.iva5fou;

class Code Region Schoolstatus SchoolLevel;

model DropoutNumba/YearlevelSize=StudentTeachRatio Region

Schoolstatus SchoolLevel/dist=bin type3;

repeated subject $=$ Code $/$ type $=$ exch;

run;

/** This is the StudentTeachRatio, Region and Schoollevel effetcs using the new dataset (iva5fou). TOTENROL IS NOT SIGNIFICANT

(Drop) $* * /$

proc genmod data $=$ SCHDROP.iva 5 fou;

class Code Region SchoolLevel;

model DropoutNumba/YearlevelSize=TotEnrol StudentTeachRatio Region

SchoolLevel/dist =bin type 3

repeated subject $=$ Code $/$ type $=$ exch;

run;

/** This is the StudentTeachRatio, Region and Schoollevel effetcs using the new dataset (iva5fou). SCHOOLLEVEL IS NOT SIGNIFICANT $($ Drop) $* * /$

proc genmod data $=$ SCHDROP.iva 5 fou;

class Code Region SchoolLevel;

model DropoutNumba/YearlevelSize=StudentTeachRatio Region

SchoolLevel/dist =bin type3;

repeated subject $=$ Code $/$ ype $=$ exch;

run; 


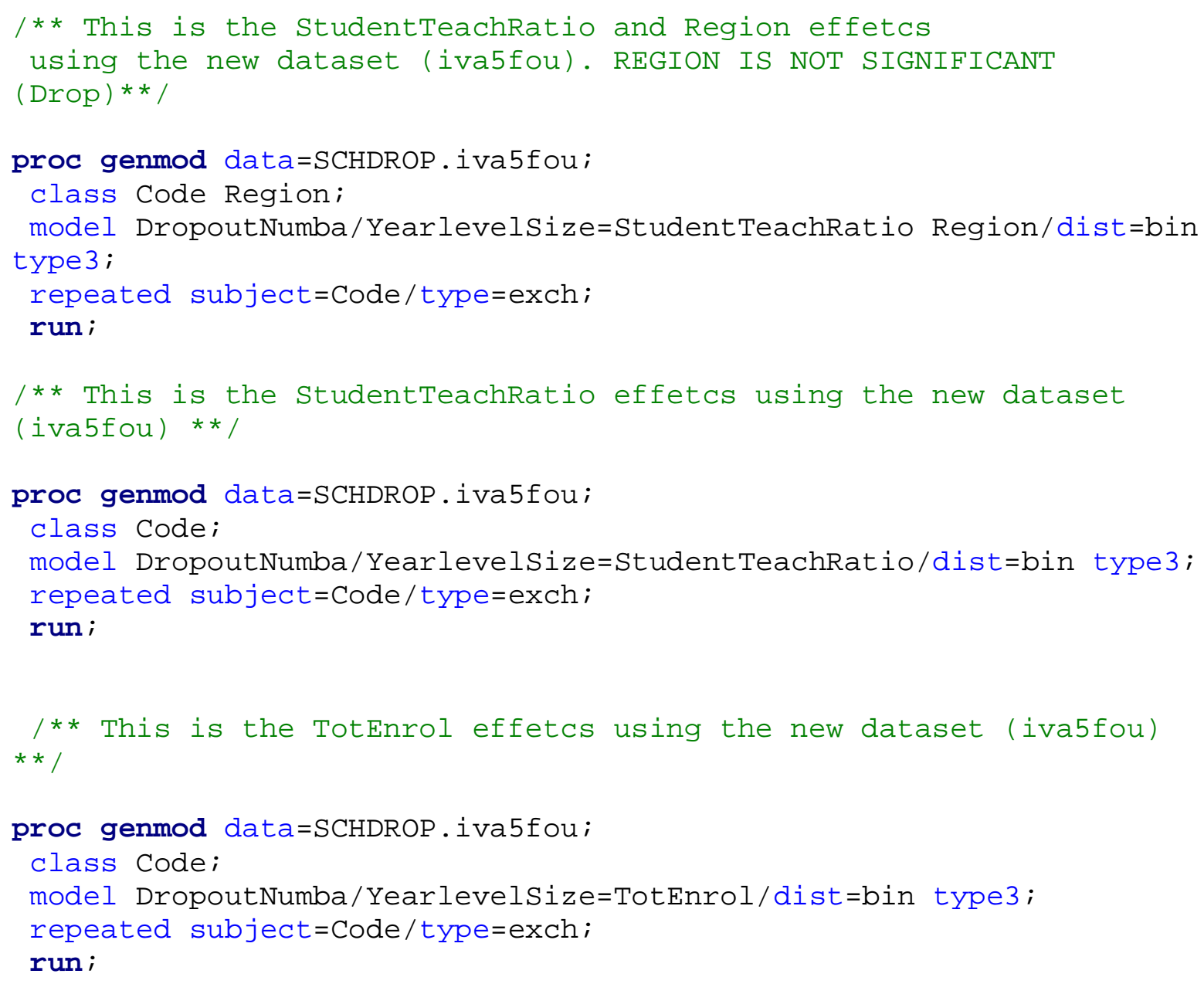

\section{MODEL CODES:}

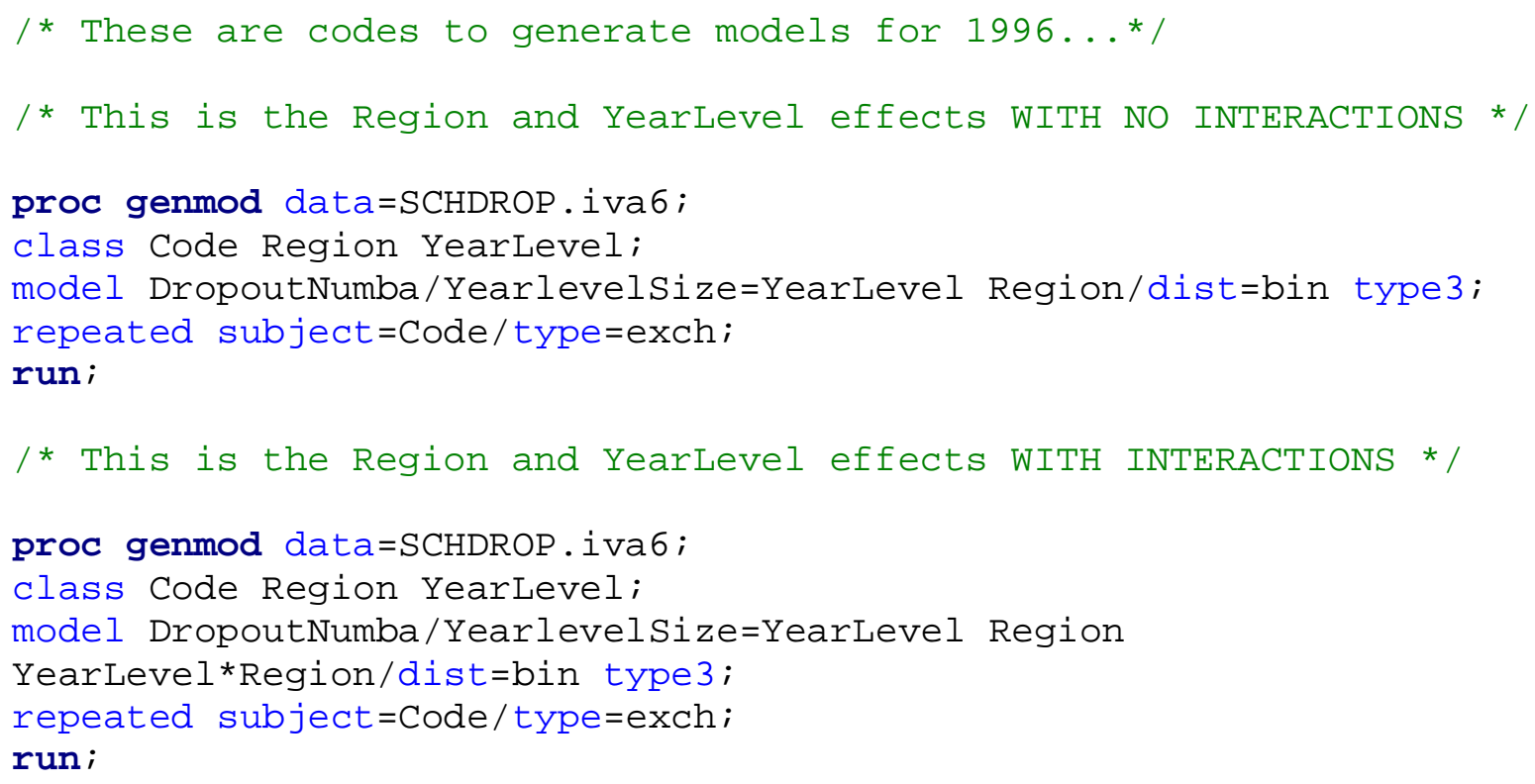


/* This is the SchoolLevel and YearLevel effects */

proc genmod data $=$ SCHDROP.iva6;

class Code SchoolLevel YearLevel;

model DropoutNumba/YearlevelSize=YearLevel SchoolLevel/dist=bin

type 3 ;

repeated subject $=$ Code $/$ type $=$ exch;

run;

/* This is the SchoolLevel and YearLevel effects WITH INTERACTIONS

[THE MODEL DID NOT CONVERGE SO USE ORDINARY LOGISTIC REGRESSION] * /

proc genmod data $=$ SCHDROP.iva6;

class Code SchoolLevel YearLevel;

model DropoutNumba/YearlevelSize=YearLevel SchoolLevel

YearLevel*SchoolLevel/dist=bin type3;

run;

/* This the Schoolstatus and YearLevel effects */

proc genmod data $=$ SCHDROP.iva 6 ;

class Code Schoolstatus YearLevel;

model DropoutNumba/YearlevelSize=YearLevel Schoolstatus/dist=bin

type 3 ;

repeated subject $=$ Code $/$ type $=$ exch;

run;

/* This is the Schoolstatus and YearLevel effects WITH INTERACTIONS [THIS MODEL DID NOT CONVERGE SO USE ORDINARY LOGISTIC REGRESSION]* /

proc genmod data $=$ SCHDROP.iva 6 ;

class Code Schoolstatus YearLevel;

model DropoutNumba/YearlevelSize=YearLevel Schoolstatus

YearLevel*Schoolstatus/dist=bin type3;

run;

/** This is the Schoolsize (TotEnrol), StudentTeachRatio, Region, Schoolstatus and SchoolLevel effetcs

using the new dataset (iva6fou). TOTENROL IS NOT SIGNIFICANT

(Drop) $* \star /$

proc genmod data=SCHDROP.iva 6 fou;

class Code Region Schoolstatus Schoollevel;

model DropoutNumba/YearlevelSize=TotEnrol StudentTeachRatio Region

Schoolstatus SchoolLevel/dist=bin type3;

repeated subject $=$ Code $/$ type $=$ exch;

run;

/** This is the StudentTeachRatio, Region, Schoolstatus and

Schoollevel effetcs

using the new dataset (iva6fou). SCHOOLSTATUS IS NOT SIGNIFICANT

(Drop) $* \star /$ 


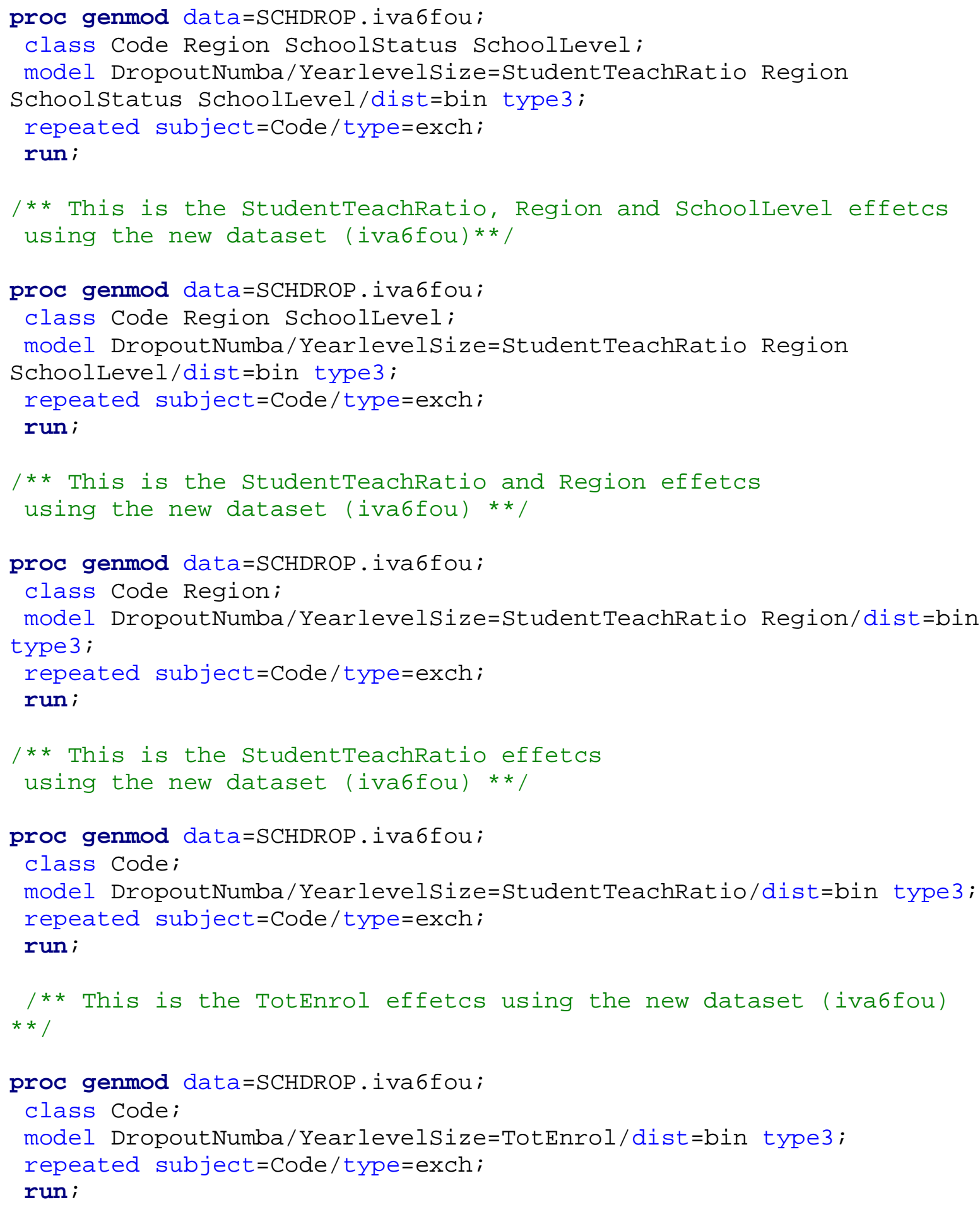

\section{MODEL CODES:}

/* These are codes to generate models for 1997...*/ 
/* This the Region and YearLevel effects WITH NO INTERACTIONS */

proc genmod data $=$ SCHDROP. iva 7 ;

class Code Region YearLevel;

model DropoutNumba/YearlevelSize=YearLevel Region/dist=bin type3; repeated subject $=$ Code $/$ type $=$ exch;

run;

/* This is the Region and YearLevel effects WITH INTERACTIONS */

proc genmod data $=$ SCHDROP.iva 7;

class Code Region YearLevel;

model DropoutNumba/YearlevelSize=YearLevel Region

YearLevel*Region/dist=bin type3;

repeated subject $=$ Code $/$ type $=$ exch;

run;

/* This is the SchoolLevel and YearLevel effects */

proc genmod data $=$ SCHDROP .iva 7;

class Code SchoolLevel YearLevel;

model DropoutNumba/Yearlevelsize=YearLevel SchoolLevel/dist=bin

type 3;

repeated subject $=$ Code $/$ type $=$ exch;

run;

/* This is the SchoolLevel and YearLevel effects WITH INTERACTIONS

[THE MODEL DID NOT CONVERGE SO USE ORDINARY LOGISTIC REGRESSION] *

proc genmod data $=$ SCHDROP.iva 7 ;

class Code SchoolLevel YearLevel;

model DropoutNumba/YearlevelSize=YearLevel SchoolLevel

YearLevel *SchoolLevel/dist=bin type3;

run;

/* This is the Schoolstatus and YearLevel effects */

proc genmod data $=$ SCHDROP.iva7;

class Code Schoolstatus YearLevel;

model DropoutNumba/YearlevelSize=YearLevel Schoolstatus/dist=bin

type 3;

repeated subject $=$ Code $/$ type $=$ exch;

run;

/* This is the Schoolstatus and YearLevel effects WITH INTERACTIONS

[THE MODEL DID NOT CONVERGE SO USE ORDINARY LOGISTIC REGRESSION]*/

proc genmod data $=$ SCHDROP.iva 7 ;

class Code Schoolstatus YearLevel;

model DropoutNumba/YearlevelSize=YearLevel Schoolstatus

YearLevel*Schoolstatus/dist=bin type3;

run; 


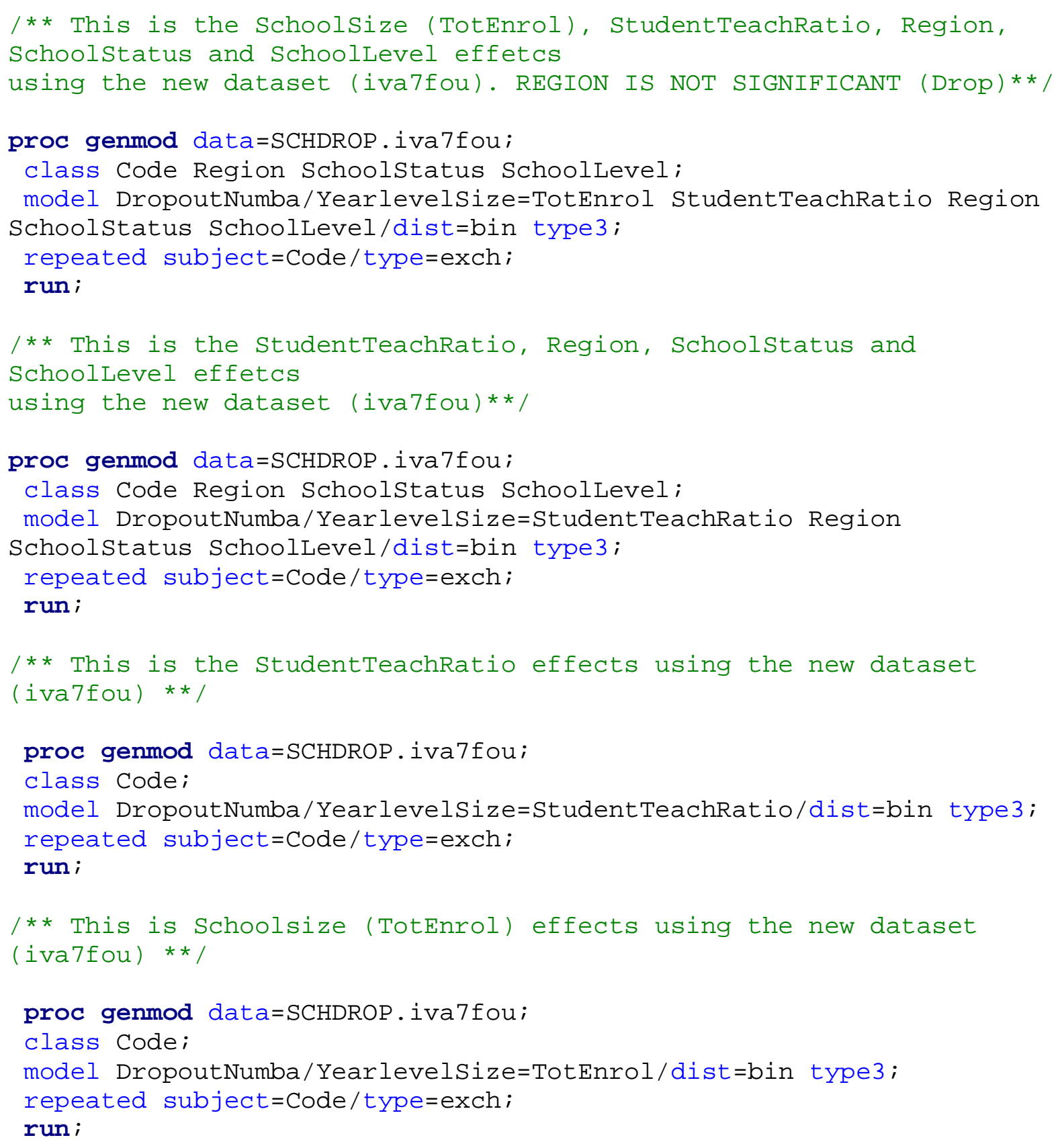

\section{MODEL CODES:}

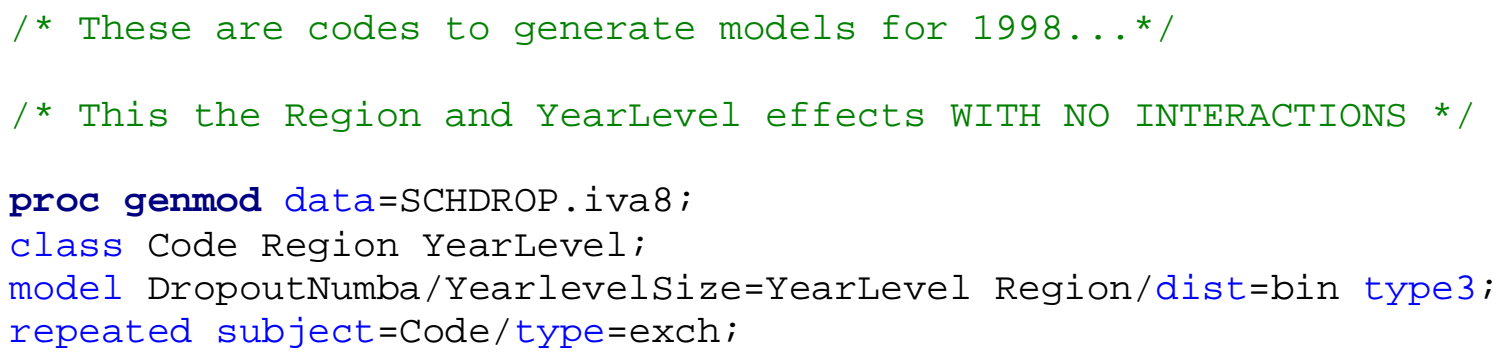


run;

/* This is the Region and YearLevel effects WITH INTERACTIONS */

proc genmod data=SCHDROP.iva 8 ;

class Code Region YearLevel;

model DropoutNumba/YearlevelSize=YearLevel Region

YearLevel*Region/dist=bin type3;

repeated subject $=$ Code $/$ type $=$ exch;

run;

/* This is the SchoolLevel and YearLevel effects */

proc genmod data $=$ SCHDROP.iva 8 ;

class Code SchoolLevel YearLevel;

model DropoutNumba/YearlevelSize=YearLevel SchoolLevel/dist=bin

type 3;

repeated subject $=$ Code $/$ type $=$ exch;

run;

/* This is the SchoolLevel and YearLevel effects WITH INTERACTIONS

[THE MODEL DID NOT CONVERGE SO USE ORDINARY LOGISTIC REGRESSION]*

proc genmod data $=$ SCHDROP.iva

class Code SchoolLevel YearLevel;

model DropoutNumba/YearlevelSize=YearLevel SchoolLevel

YearLevel*SchoolLevel/dist=bin type3;

run;

/* This is the Schoolstatus and YearLevel effects */

proc genmod data $=$ SCHDROP.iva 8

class Code Schoolstatus YearLevel;

model DropoutNumba/YearlevelSize=YearLevel Schoolstatus/dist=bin

type 3;

repeated subject $=$ Code $/$ type $=$ exch;

run;

/* This is the Schoolstatus and YearLevel effects WITH INTERACTIONS

$\star /$

proc genmod data=SCHDROP.iva 8 ;

class Code Schoolstatus YearLevel;

model DropoutNumba/YearlevelSize=YearLevel Schoolstatus

YearLevel*Schoolstatus/dist=bin type3;

repeated subject $=$ Code $/$ type $=$ exch;

run;

/** This is the Schoolsize (TotEnrol), StudentTeachRatio, Region, Schoolstatus and Schoollevel effetcs

using the new dataset (iva8fou). TOTENROL IS NOT SIGNIFICANT

(Drop) $* * /$

proc genmod data $=$ SCHDROP.iva 8 fou; 
class Code Region Schoolstatus SchoolLevel;

model DropoutNumba/YearlevelSize=TotEnrol StudentTeachRatio Region

Schoolstatus SchoolLevel/dist=bin type3;

repeated subject $=$ Code $/$ type $=$ exch;

run;

/** This is the StudentTeachRatio, Region, Schoolstatus and

Schoollevel effetcs

using the new dataset (iva8fou). SCHOOLLEVEL IS NOT SIGNIFICANT

(Drop) $* * /$

proc genmod data $=$ SCHDROP.iva8fou;

class Code Region Schoolstatus SchoolLevel;

model DropoutNumba/YearlevelSize=StudentTeachRatio Region

Schoolstatus SchoolLevel/dist=bin type3;

repeated subject $=$ Code $/$ type $=$ exch;

run;

/** This is the StudentTeachRatio, Region and Schoolstatus effetcs using the new dataset (iva8fou). REGION IS NOT SIGNIFICANT (Drop)**/

proc genmod data=SCHDROP.iva8fou;

class Code Region Schoolstatus;

model DropoutNumba/YearlevelSize=StudentTeachRatio Region

Schoolstatus/dist=bin type3;

repeated subject $=$ Code $/$ type $=$ exch;

run;

/** This is the StudentTeachRatio and Schoolstatus effetcs using the new dataset (iva8fou). SCHOOLSTATUS IS NOT SIGNIFICANT (Drop) $* * /$

proc genmod data $=$ SCHDROP.iva8fou;

class Code Schoolstatus;

model DropoutNumba/YearlevelSize=StudentTeachRatio

Schoolstatus/dist=bin type 3 ;

repeated subject $=$ Code $/$ type $=$ exch;

run;

/** This is the StudentTeachRatio effetcs using the new dataset (iva8fou) $* \star /$

proc genmod data $=$ SCHDROP.iva8fou;

class Code;

model DropoutNumba/YearlevelSize=StudentTeachRatio/dist=bin type3; repeated subject $=$ Code $/$ type $=$ exch;

run;

/** This is Schoolsize (TotEnrol) effects using the new dataset

(iva8fou) $\star \star / /$

proc genmod data=SCHDROP.iva8fou;

class Code;

model DropoutNumba/YearlevelSize=TotEnrol/dist=bin type3;

repeated subject $=$ Code $/$ type $=$ exch;

run; 


\section{MODEL CODES:}

/* These are codes to generate models for $1999 . .$. /
/* This the Region and YearLevel effects WITH NO INTERACTIONS */

proc genmod data=SCHDROP.iva9;

class Code Region YearLevel;

model DropoutNumba/YearlevelSize=YearLevel Region/dist=bin type3; repeated subject $=$ code/type $=$ exch;

run;

/* This is the Region and YearLevel effects WITH INTERACTIONS */

proc genmod data=SCHDROP.iva9;

class Code Region YearLevel;

model DropoutNumba/YearlevelSize=YearLevel Region

YearLevel *Region/dist=bin type3;

repeated subject $=$ Code $/$ type $=$ exch;

run;

/* This is the SchoolLevel and YearLevel effects */

proc genmod data=SCHDROP.iva9;

class Code SchoolLevel YearLevel;

model DropoutNumba/YearlevelSize=YearLevel SchoolLevel/dist=bin

type 3;

repeated subject $=$ Code $/$ type $=$ exch;

run;

/* This is the Schoollevel and YearLevel effects WITH INTERACTIONS

[THE MODEL DID NOT CONVERGE SO USE ORDINARY LOGISTIC REGRESSION]*/

proc genmod data=SCHDROP.iva9;

class Code SchoolLevel YearLevel;

model DropoutNumba/YearlevelSize=YearLevel SchoolLevel

YearLevel *SchoolLevel/dist=bin type3;

run;

/* This is the Schoolstatus and YearLevel effects */

proc genmod data=SCHDROP.iva9;

class Code Schoolstatus YearLevel;

model DropoutNumba/YearlevelSize=YearLevel Schoolstatus/dist=bin

type 3;

repeated subject $=$ code $/$ type $=$ exch;

run;

/* This is the Schoolstatus and YearLevel effects WITH INTERACTIONS 


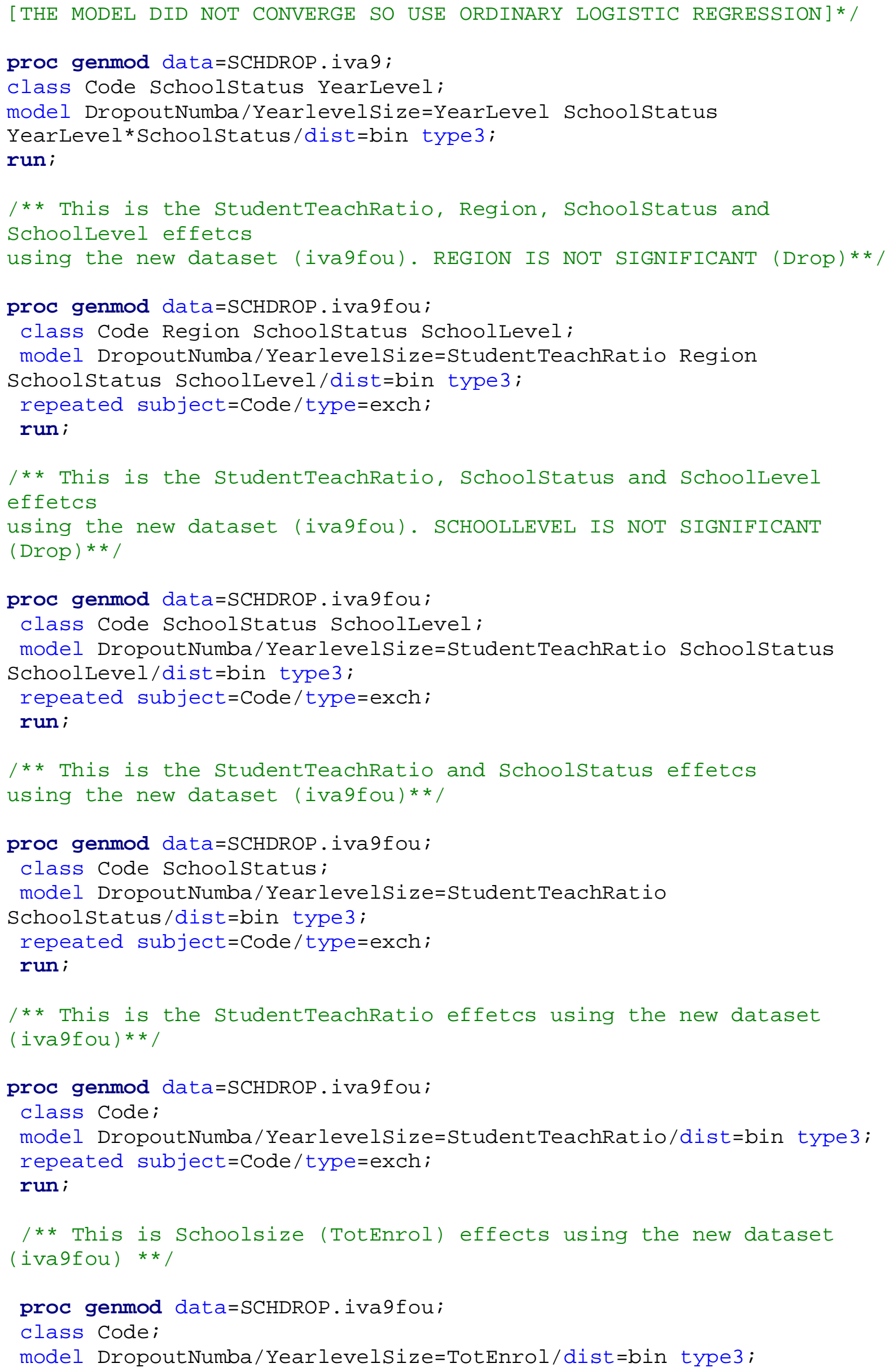




\section{MODEL CODES:}

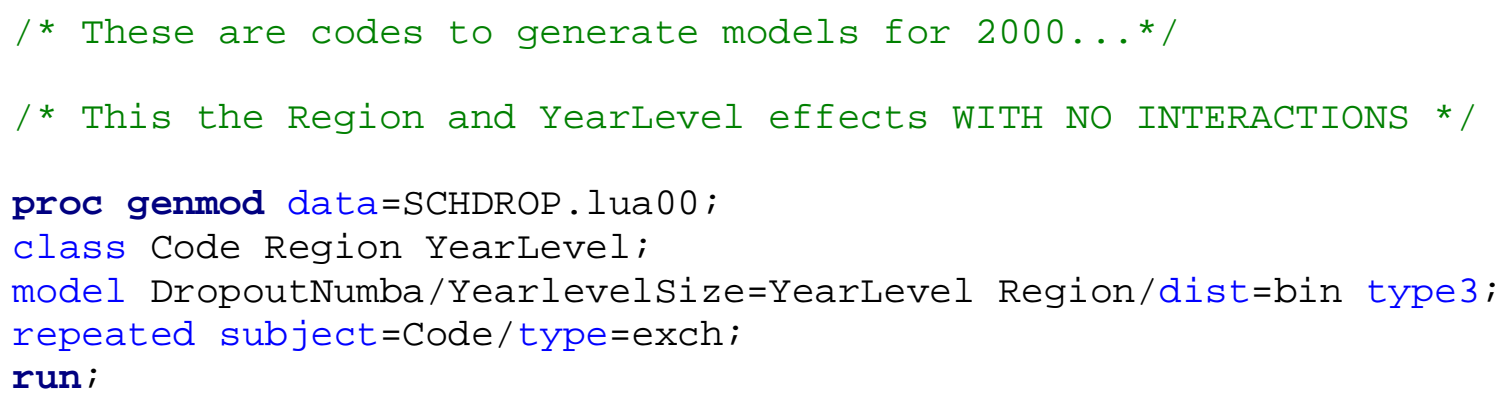


run;

/* This is the Schoolstatus and YearLevel effects WITH INTERACTIONS [THE MODEL DID NOT CONVERGE SO USE ORDINARY LOGISTIC REGRESSION] */

proc genmod data $=$ SCHDROP. luaO0;

class Code Schoolstatus YearLevel;

model DropoutNumba/YearlevelSize=YearLevel Schoolstatus

YearLevel*Schoolstatus/dist=bin type3;

run;

/** This is the StudentTeachRatio, Region, Schoolstatus and

Schoollevel effetcs

using the new dataset (luaOOfou). SCHOOLLEVEL IS NOT SIGNIFICANT

(Drop) $* * /$

proc genmod data $=$ SCHDROP.luaO0 fou;

class Code Region Schoolstatus SchoolLevel;

model Dropout Numba/YearlevelSize=StudentTeachRatio Region

Schoolstatus SchoolLevel/dist=bin type3;

repeated subject $=$ Code $/$ type $=$ exch;

run;

/** This is the StudentTeachRatio, Region and Schoolstatus effetcs using the new dataset (luaOOfou). SCHOOLSTATUS IS NOT SIGNIFICANT $($ Drop $) * * /$

proc genmod data $=$ SCHDROP.lua00fou;

class Code Region Schoolstatus;

model DropoutNumba/YearlevelSize=StudentTeachRatio Region

Schoolstatus/dist=bin type3;

repeated subject $=$ Code $/$ type $=$ exch;

run;

/** This is the StudentTeachRatio and Region effetcs

using the new dataset (luaOofou)**/

proc genmod data $=$ SCHDROP.luaOOfou;

class Code Region;

model DropoutNumba/YearlevelSize=StudentTeachRatio Region/dist=bin

type 3

repeated subject $=$ Code $/$ type $=$ exch;

run;

/** This is the StudentTeachRatio effetcs using the new dataset

(lua 00 fou $) * * /$

proc genmod data $=$ SCHDROP.lua00fou;

class Code;

model DropoutNumba/YearlevelSize=StudentTeachRatio/dist=bin type3; repeated subject $=$ Code $/$ type $=$ exch;

run;

/** This is Schoolsize (TotEnrol) effects using the new dataset

(lua00fou) $* * /$ 
proc genmod data $=$ SCHDROP. lua00fou;

class Code;

model DropoutNumba/YearlevelSize=TotEnrol/dist=bin type3;

repeated subject $=$ Code $/$ type $=$ exch;

run;

\section{MODEL CODES:}

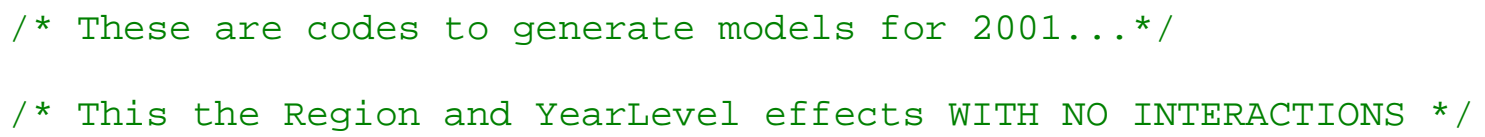


model DropoutNumba/YearlevelSize=YearLevel Schoolstatus/dist=bin type 3;

repeated subject $=$ Code $/$ type $=$ exch;

run;

/* This is the Schoolstatus and YearLevel effects WITH INTERACTIONS [THE MODEL DID NOT CONVERGE SO USE ORDINARY LOGISTIC REGRESSION] */

proc genmod data=SCHDROP.lua01;

class Code Schoolstatus YearLevel;

model DropoutNumba/YearlevelSize=YearLevel Schoolstatus

YearLevel*Schoolstatus/dist=bin type3;

run;

/** This is the StudentTeachRatio, Region, Schoolstatus and

Schoollevel effetcs

using the new dataset (lua01fou). SCHOOLSTATUS IS NOT SIGNIFICANT

(Drop) $* * /$

proc genmod data $=$ SCHDROP. lua01fou;

class Code Region Schoolstatus SchoolLevel;

model DropoutNumba/YearlevelSize=StudentTeachRatio Region

Schoolstatus SchoolLevel/dist=bin type3;

repeated subject $=$ Code $/$ ype $=$ exch;

run;

/** This is the StudentTeachRatio, Region and Schoollevel effetcs using the new dataset (luaOlfou). SCHOOLLEVEL IS NOT SIGNIFICANT

(Drop) $* * /$

proc genmod data $=$ SCHDROP. lua01fou;

class Code Region SchoolLevel;

model DropoutNumba/YearlevelSize=StudentTeachRatio Region

Schoollevel/dist=bin type3;

repeated subject $=$ Code $/$ type $=$ exch;

run;

/** This is the StudentTeachRatio and Region effetcs

using the new dataset (lua01fou)**/

proc genmod data $=$ SCHDROP.lua01fou;

class Code Region;

model DropoutNumba/YearlevelSize=StudentTeachRatio Region/dist=bin

type 3;

repeated subject $=$ Code $/$ type $=$ exch;

run;

/** This is the StudentTeachRatio effetcs using the new dataset (lua01fou) $* * /$

proc genmod data=SCHDROP.lua01fou;

class Code;

model DropoutNumba/YearlevelSize=StudentTeachRatio/dist=bin type3; repeated subject $=$ Code $/$ type $=$ exch;

run; 
/** This is Schoolsize (TotEnrol) effects using the new dataset

(lua01fou) $* * /$

proc genmod data $=$ SCHDROP. lua01fou;

class Code;

model DropoutNumba/YearlevelSize=TotEnrol/dist=bin type3;

repeated subject $=$ Code $/$ type $=$ exch;

run;

\section{MODEL CODES:}

/* These are codes to generate models for 2002...*/

/* This the Region and YearLevel effects WITH NO INTERACTIONS */

proc genmod data=SCHDROP.lua02;

class Code Region YearLevel;

model DropoutNumba/YearlevelSize=YearLevel Region/dist=bin type3; repeated subject $=$ Code $/$ type $=$ exch;

run;

/* This is the Region and YearLevel effects WITH INTERACTIONS */

proc genmod data=SCHDROP.lua02;

class Code Region YearLevel;

model DropoutNumba/YearlevelSize=YearLevel Region

YearLevel*Region/dist=bin type3;

repeated subject $=$ Code $/$ type $=$ exch;

run;

/* This is the SchoolLevel and YearLevel effects */

proc genmod data=SCHDROP.lua02;

class Code SchoolLevel YearLevel;

model DropoutNumba/YearlevelSize=YearLevel SchoolLevel/dist=bin

type 3;

repeated subject $=$ Code $/$ type $=$ exch;

run;

/* This is the SchoolLevel and YearLevel effects WITH INTERACTIONS

[THE MODEL DID NOT CONVERGE SO USE ORDINARY LOGISTIC REGRESSION]*/

proc genmod data=SCHDROP.lua02;

class Code SchoolLevel YearLevel;

model DropoutNumba/YearlevelSize=YearLevel SchoolLevel

YearLevel *SchoolLevel/dist=bin type3;

run; 


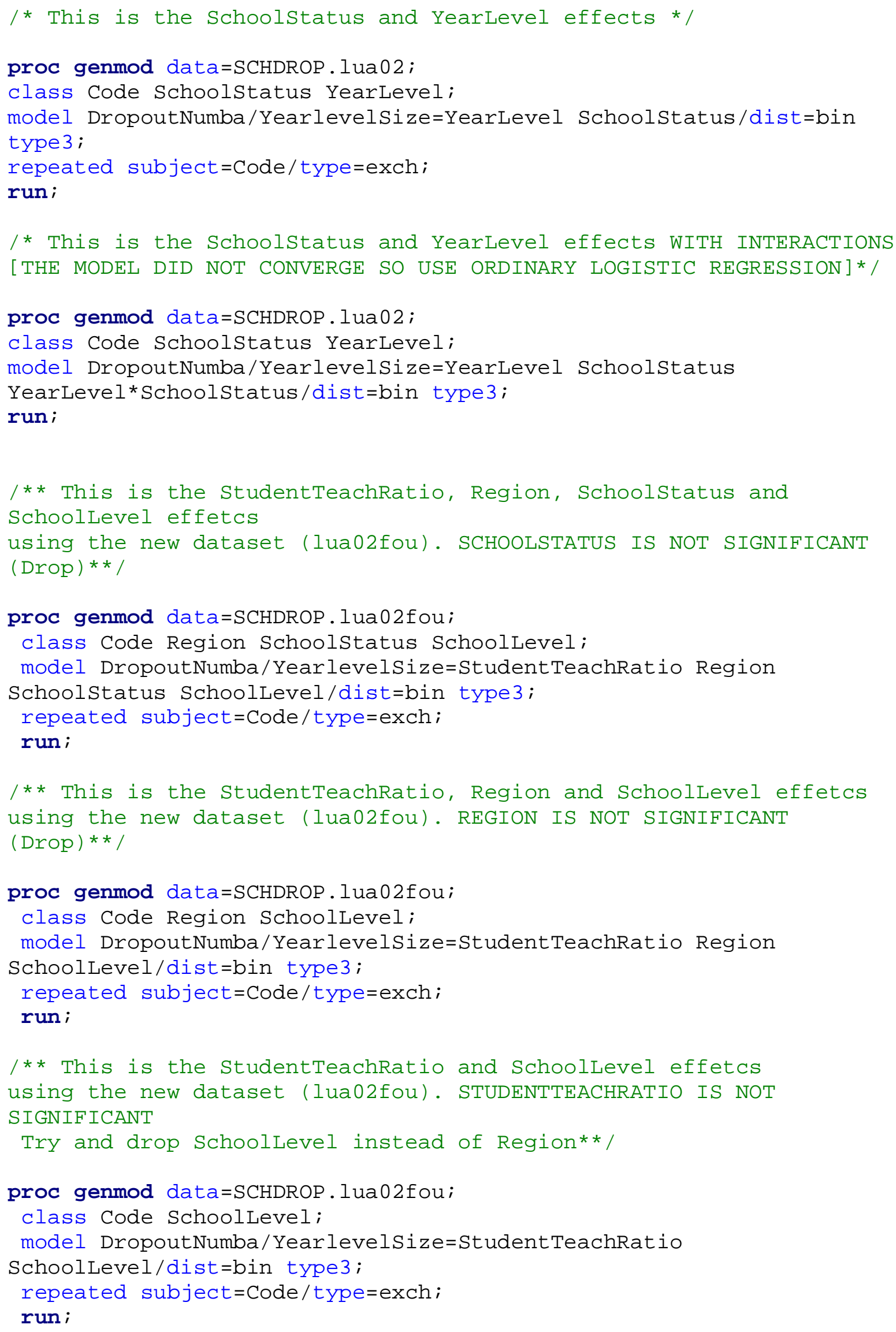




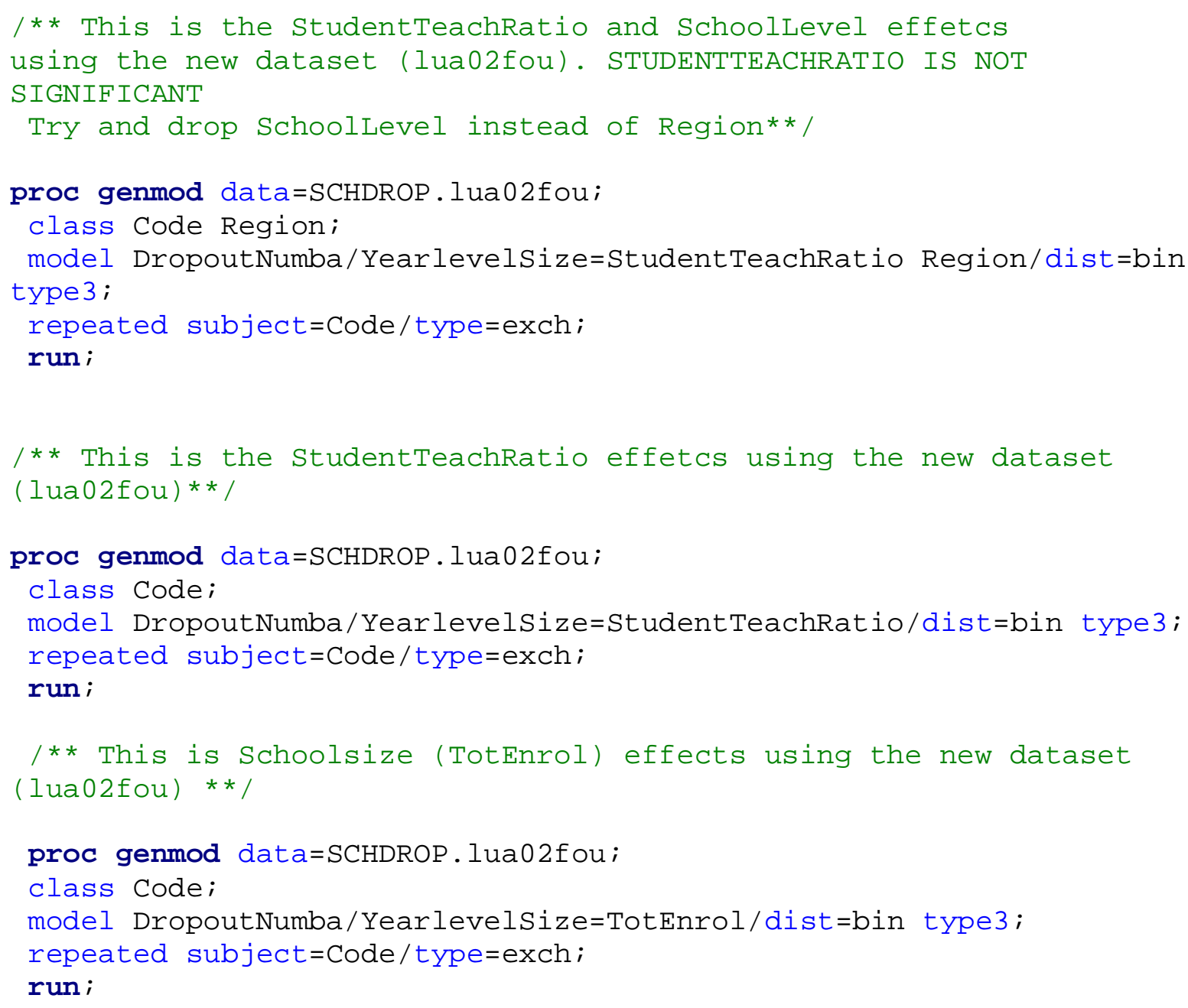

\section{MODEL CODES:}

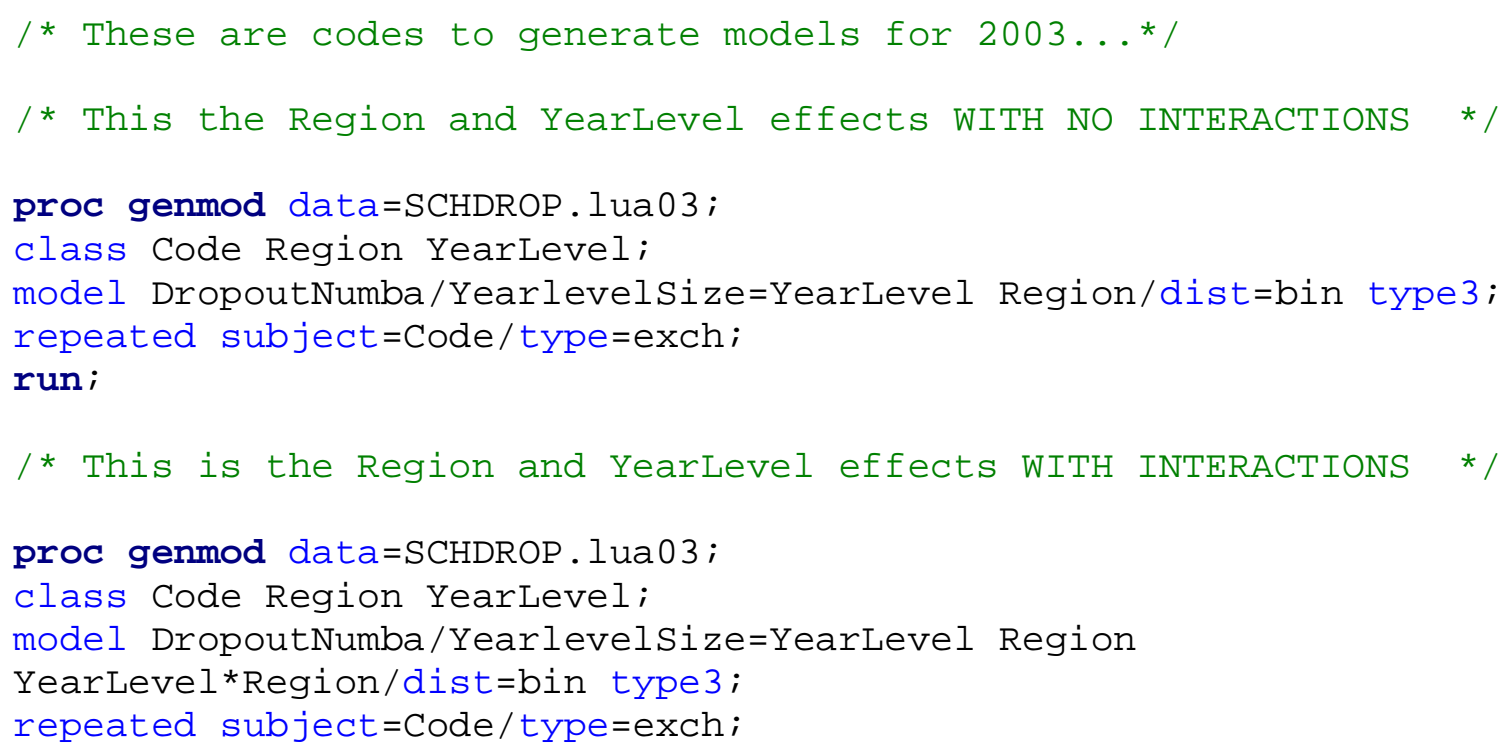


/* This is the SchoolLevel and YearLevel effects */

proc genmod data $=$ SCHDROP.lua03;

class Code SchoolLevel YearLevel;

model DropoutNumba/YearlevelSize=YearLevel SchoolLevel/dist=bin

type 3;

repeated subject $=$ Code $/$ type $=$ exch;

run;

/* This is the SchoolLevel and YearLevel effects WITH INTERACTIONS [THE MODEL DID NOT CONVERGE SO USE ORDINARY LOGISTIC REGRESSION]* /

proc genmod data $=$ SCHDROP.lua03;

class Code SchoolLevel YearLevel;

model DropoutNumba/YearlevelSize=YearLevel SchoolLevel

YearLevel *SchoolLevel/dist=bin type3;

run;

/* This is the Schoolstatus and YearLevel effects */

proc genmod data $=$ SCHDROP.lua03;

class Code Schoolstatus YearLevel;

model DropoutNumba/YearlevelSize=YearLevel Schoolstatus/dist=bin

type 3;

repeated subject $=$ Code $/$ type $=$ exch;

run;

/* This is the Schoolstatus and YearLevel effects WITH INTERACTIONS [THE MODEL DID NOT CONVERGE SO USE ORDINARY LOGISTIC REGRESSION]*/

proc genmod data $=$ SCHDROP.lua03;

class Code Schoolstatus YearLevel;

model DropoutNumba/YearlevelSize=YearLevel Schoolstatus

YearLevel*Schoolstatus/dist=bin type3;

run;

/** This is the StudentTeachRatio, Region, Schoolstatus and

Schoollevel effetcs

using the new dataset (lua03fou). SCHOOLSTATUS IS NOT SIGNIFICANT

$($ Drop $) * * /$

proc genmod data $=$ SCHDROP.lua03fou;

class Code Region Schoolstatus SchoolLevel;

model DropoutNumba/YearlevelSize=StudentTeachRatio Region

Schoolstatus SchoolLevel/dist=bin type3;

repeated subject $=$ Code $/$ type $=$ exch;

run;

/** This is the StudentTeachRatio, Region and Schoollevel effetcs using the new dataset (lua03fou). SCHOOLLEVEL IS NOT SIGNIFICANT (Drop) **/ 


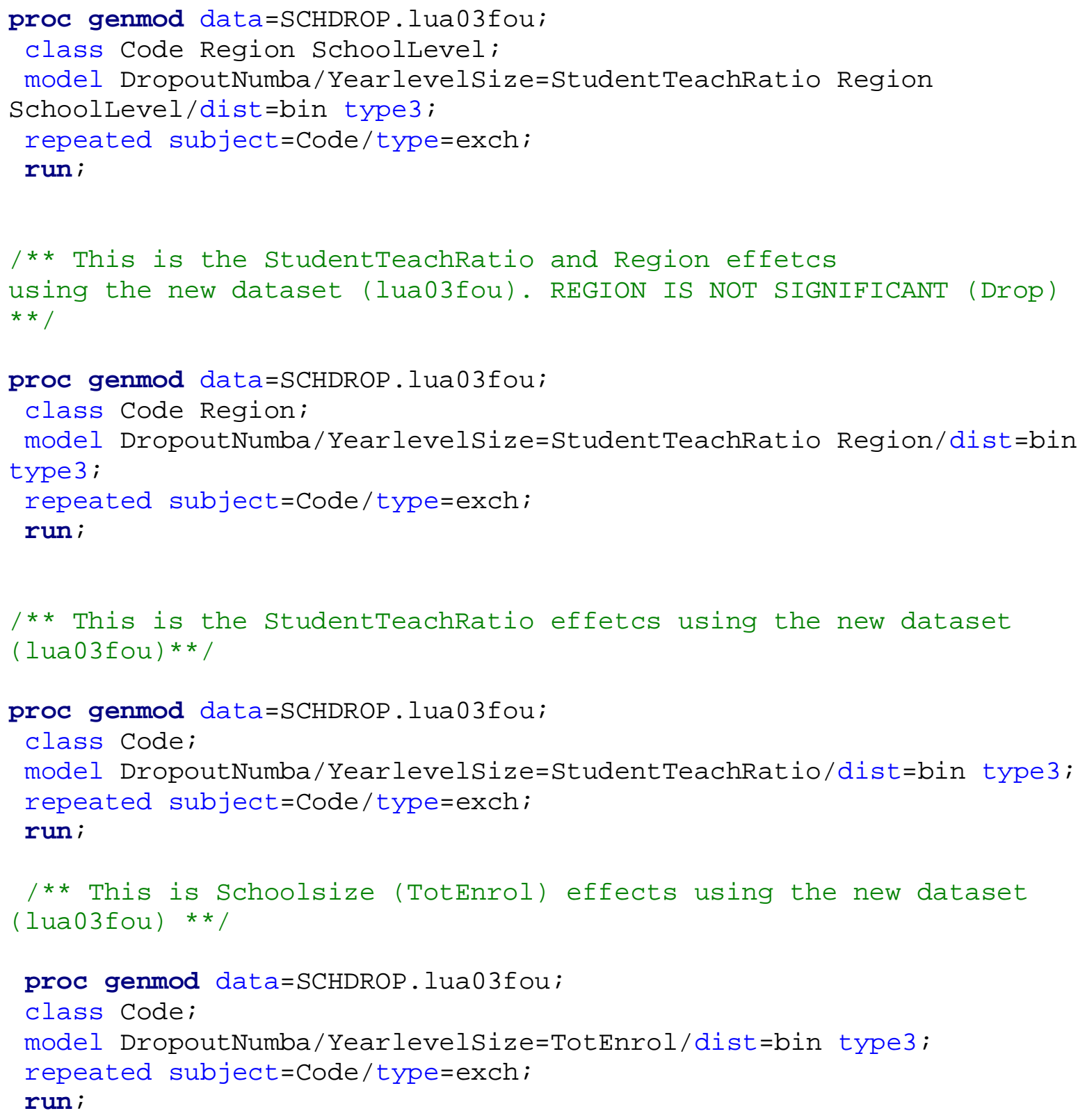

\section{MODEL CODES:}

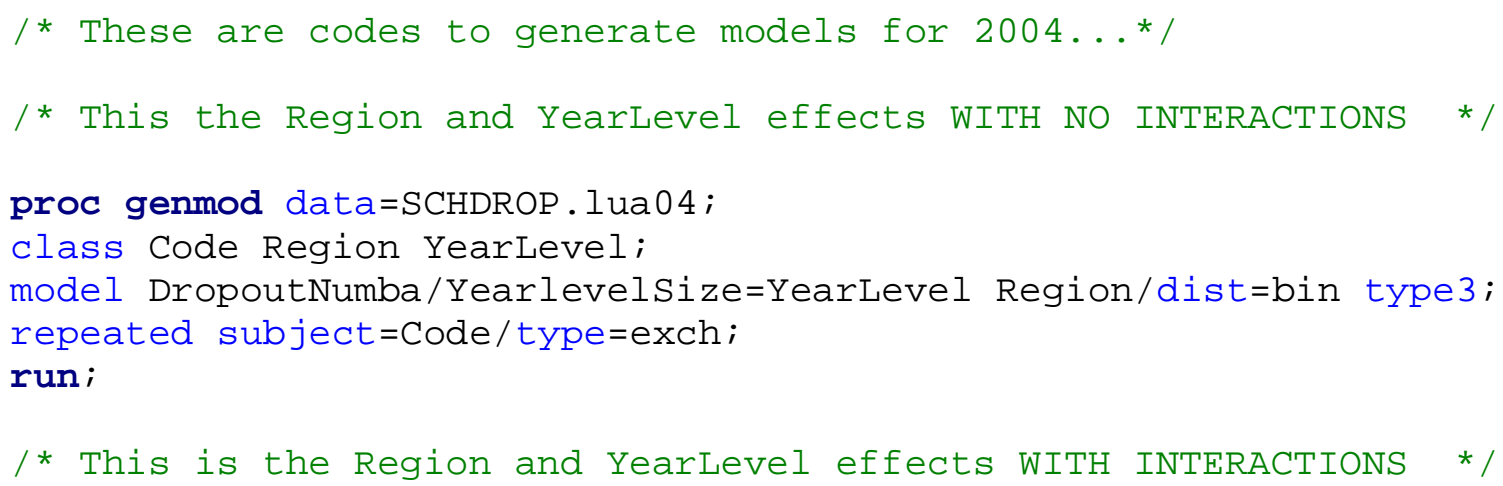


proc genmod data $=$ SCHDROP.lua04;

class Code Region YearLevel;

model DropoutNumba/YearlevelSize=YearLevel Region

YearLevel*Region/dist=bin type3;

repeated subject $=$ Code $/$ type $=$ exch;

run;

/* This is the SchoolLevel and YearLevel effects */

proc genmod data $=$ SCHDROP.lua04;

class Code SchoolLevel YearLevel;

model DropoutNumba/YearlevelSize=YearLevel SchoolLevel/dist=bin

type 3;

repeated subject $=$ Code $/$ type $=$ exch;

run;

/* This is the SchoolLevel and YearLevel effects WITH INTERACTIONS

[THE MODEL DID NOT CONVERGE SO USE ORDINARY LOGISTIC REGRESSION]*/

proc genmod data=SCHDROP.lua04;

class Code SchoolLevel YearLevel;

model DropoutNumba/YearlevelSize=YearLevel SchoolLevel

YearLevel*SchoolLevel/dist=bin type3;

run;

/* This is the Schoolstatus and YearLevel effects */

proc genmod data=SCHDROP.lua04;

class Code Schoolstatus YearLevel;

model DropoutNumba/YearlevelSize=YearLevel Schoolstatus/dist=bin

type 3;

repeated subject $=$ Code $/$ type $=$ exch;

run;

/* This is the Schoolstatus and YearLevel effects WITH INTERACTIONS

[THE MODEL DID NOT CONVERGE SO USE ORDINARY LOGISTIC REGRESSION] */

proc genmod data=SCHDROP.lua04;

class Code Schoolstatus YearLevel;

model DropoutNumba/YearlevelSize=YearLevel Schoolstatus

YearLevel*Schoolstatus/dist=bin type3;

run;

/** This is the StudentTeachRatio, Region, Schoolstatus and

Schoollevel effetcs

using the new dataset (luaO4fou). SCHOOLSTATUS IS NOT SIGNIFICANT

(Drop) $* * /$

proc genmod data $=$ SCHDROP.lua04fou;

class Code Region Schoolstatus SchoolLevel;

model DropoutNumba/YearlevelSize=StudentTeachRatio Region

Schoolstatus SchoolLevel/dist=bin type3;

repeated subject $=$ Code $/$ type $=$ exch ;

run; 
/** This is the StudentTeachRatio, Region and Schoollevel effetcs using the new dataset (lualfou) $\star * /$

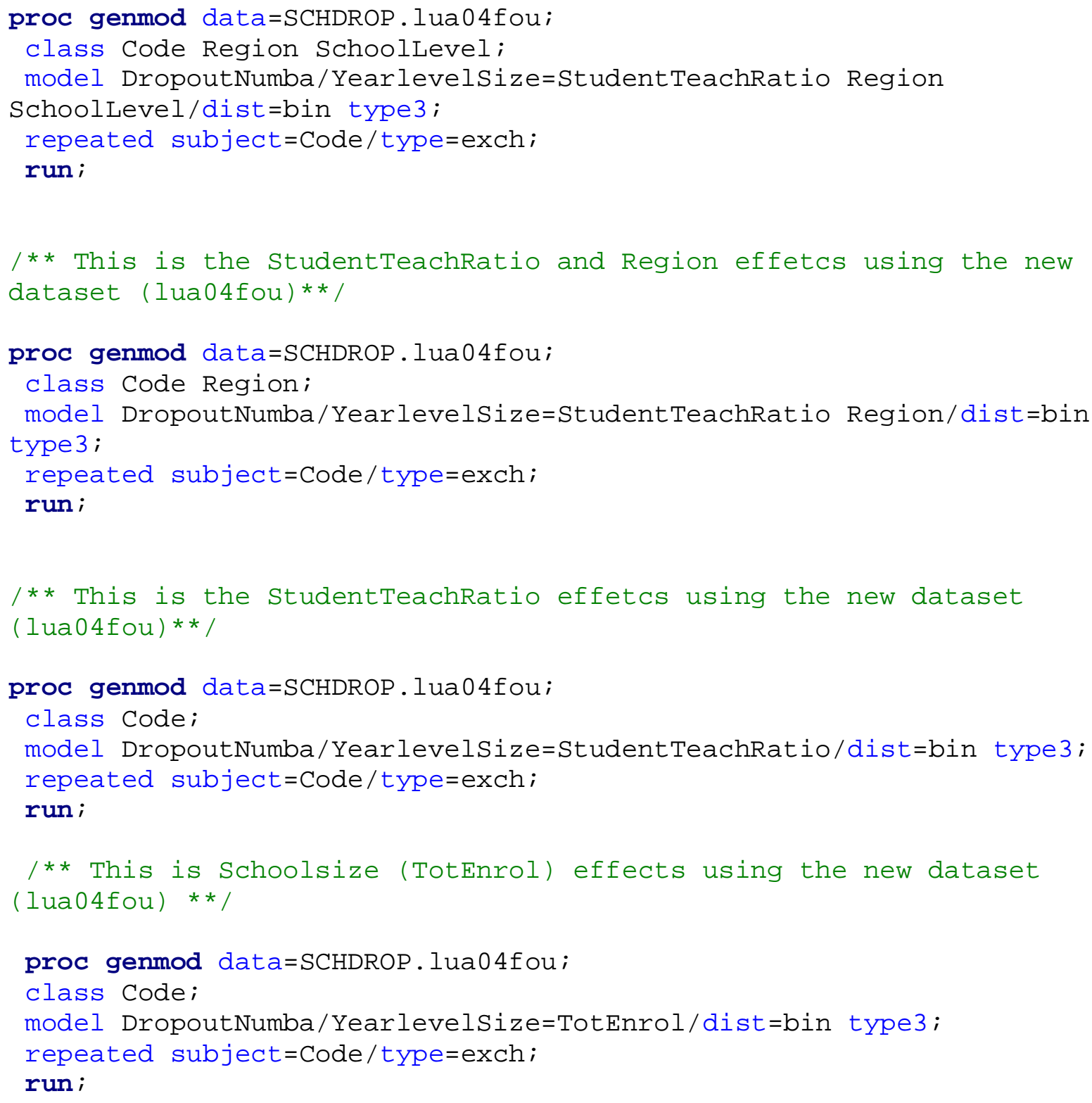

\section{MODEL CODES:}

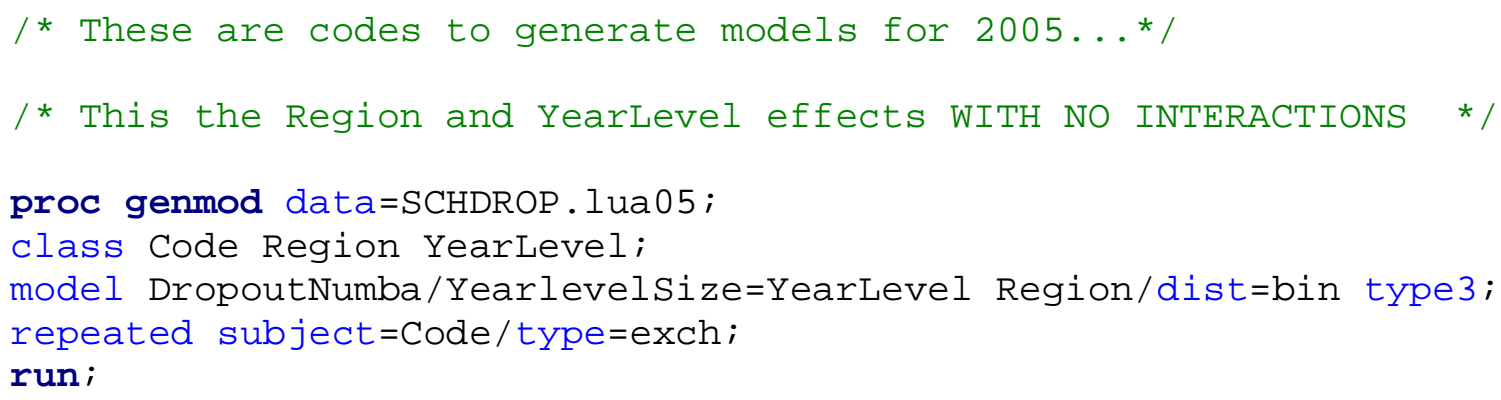


/* This is the Region and YearLevel effects WITH INTERACTIONS */

proc genmod data=SCHDROP.lua05;

class Code Region YearLevel;

model DropoutNumba/YearlevelSize=YearLevel Region

YearLevel*Region/dist=bin type3;

repeated subject $=$ Code $/$ type $=$ exch;

run;

/* This is the SchoolLevel and YearLevel effects */

proc genmod data=SCHDROP.lua05;

class Code SchoolLevel YearLevel;

model DropoutNumba/YearlevelSize=YearLevel SchoolLevel/dist=bin

type 3 ;

repeated subject $=$ Code $/$ type $=$ exch;

run;

/* This is the SchoolLevel and YearLevel effects WITH INTERACTIONS $\star /$

proc genmod data $=$ SCHDROP.lua05;

class Code SchoolLevel YearLevel;

model DropoutNumba/YearlevelSize=YearLevel SchoolLevel

YearLevel *SchoolLevel/dist=bin type3;

repeated subject $=$ Code $/$ type $=$ exch;

run;

/* This is the Schoolstatus and YearLevel effects */

proc genmod data $=$ SCHDROP.lua05;

class Code Schoolstatus YearLevel;

model DropoutNumba/YearlevelSize=YearLevel Schoolstatus/dist=bin

type 3 ;

repeated subject $=$ Code $/$ type $=$ exch;

run;

/* This is the Schoolstatus and YearLevel effects WITH INTERACTIONS

$\star /$

proc genmod data $=$ SCHDROP.lua05;

class Code Schoolstatus YearLevel;

model DropoutNumba/YearlevelSize=YearLevel Schoolstatus

YearLevel*Schoolstatus/dist=bin type3;

repeated subject $=$ Code $/$ type $=$ exch;

run;

/** This is the StudentTeachRatio, Region, Schoolstatus and

Schoollevel effetcs

using the new dataset (lua05fou). SCHOOLSTATUS IS NOT SIGNIFICANT

(Drop) $* * /$

proc genmod data $=$ SCHDROP. lua 05 fou; 


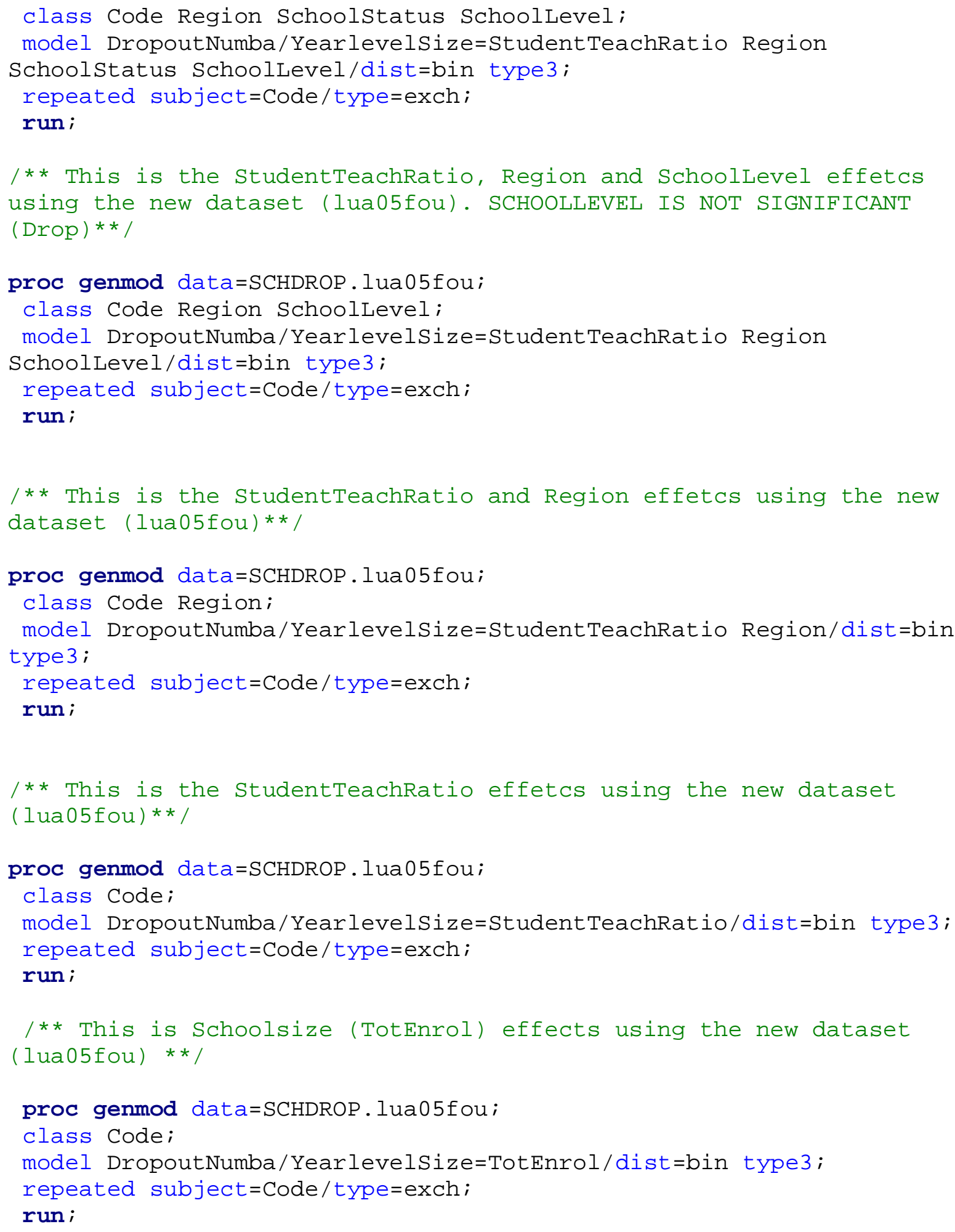

\section{MODEL CODES:}

/* These are codes to generate models for 2006..*/ 
/* This the Region and YearLevel effects WITH NO INTERACTIONS */

proc genmod data=SCHDROP.lua06;

class Code Region YearLevel;

model DropoutNumba/YearlevelSize=YearLevel Region/dist=bin type3; repeated subject $=$ Code $/$ type $=$ exch;

run;

/* This is the Region and YearLevel effects WITH INTERACTIONS */

proc genmod data=SCHDROP.lua06;

class Code Region YearLevel;

model DropoutNumba/YearlevelSize=YearLevel Region

YearLevel*Region/dist=bin type3;

repeated subject $=$ Code $/$ type $=$ exch;

run;

/* This is the SchoolLevel and YearLevel effects */

proc genmod data $=$ SCHDROP.lua06;

class Code SchoolLevel YearLevel;

model DropoutNumba/YearlevelSize=YearLevel SchoolLevel/dist=bin

type 3;

repeated subject $=$ Code $/$ type $=$ exch;

run;

/* This is the SchoolLevel and YearLevel effects WITH INTERACTIONS

[THE MODEL DID NOT CONVERGE SO USE ORDINARY LOGISTIC REGRESSION] */

proc genmod data=SCHDROP.lua06;

class Code SchoolLevel YearLevel;

model DropoutNumba/YearlevelSize=YearLevel SchoolLevel

YearLevel*SchoolLevel/dist=bin type3;

run;

/* This is the Schoolstatus and YearLevel effects */

proc genmod data=SCHDROP.lua06;

class Code Schoolstatus YearLevel;

model DropoutNumba/YearlevelSize=YearLevel Schoolstatus/dist=bin

type 3;

repeated subject $=$ Code $/$ type $=$ exch;

run;

/* This is the Schoolstatus and YearLevel effects WITH INTERACTIONS

[THE MODEL DID NOT CONVERGE SO USE ORDINARY LOGISTIC REGRESSION]* /

proc genmod data=SCHDROP.lua06;

class Code Schoolstatus YearLevel;

model DropoutNumba/YearlevelSize=YearLevel Schoolstatus

YearLevel*Schoolstatus/dist=bin type3;

run; 


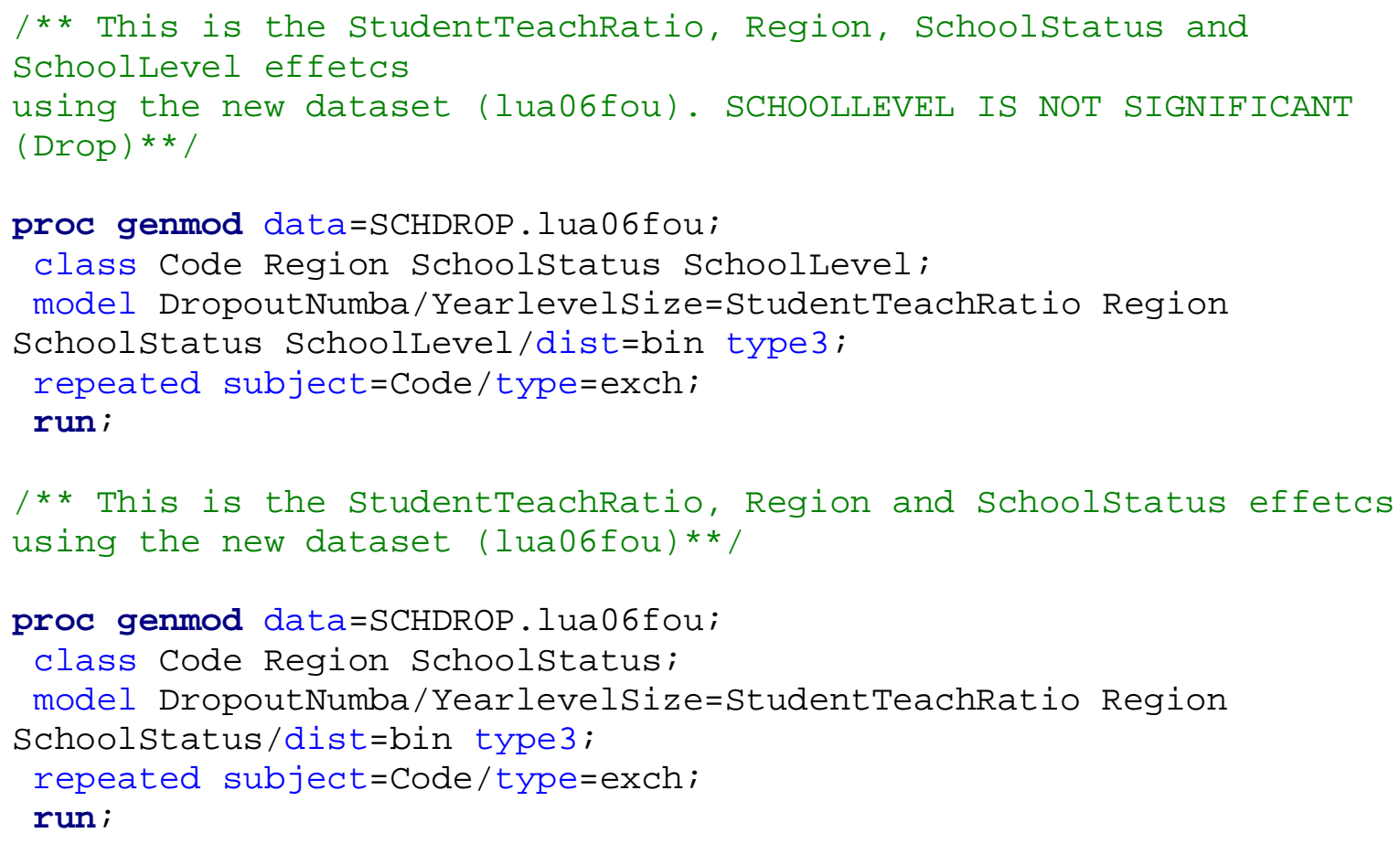




\section{THESE ARE CODES FOR SECONDARY SCHOOLS DATA ONLY FOR THE YEAR 2007 ONLY:}

\section{MODEL CODES:}

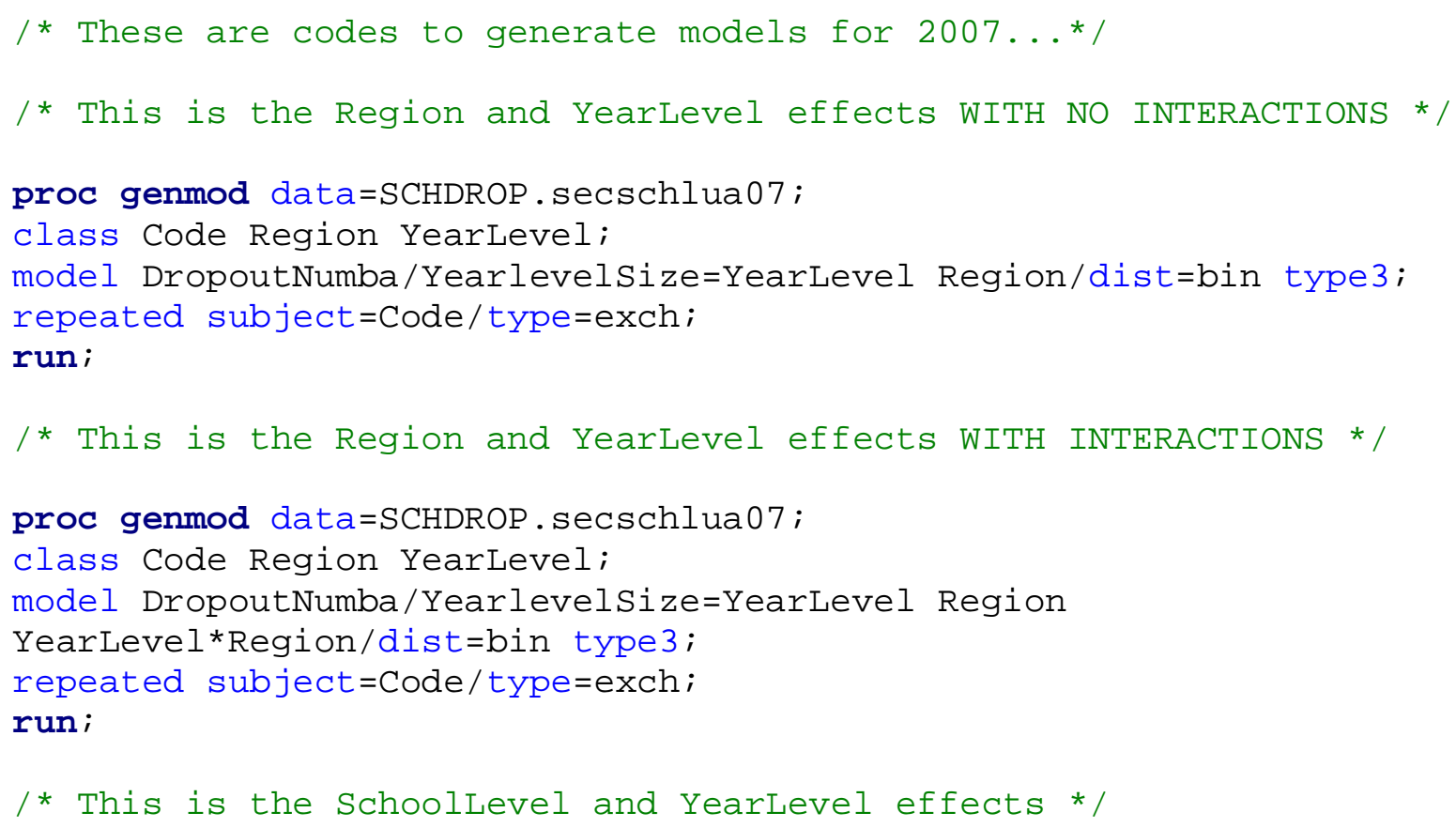


proc genmod data=SCHDROP. secschlua07;

class Code Schoolstatus YearLevel; model DropoutNumba/YearlevelSize=YearLevel Schoolstatus YearLevel*Schoolstatus/dist=bin type3; run;

/** This is the StudentTeachRatio, Region, Schoolstatus and Schoollevel effetcs SCHOOLSTATUS IS NOT SIGNIFICANT (Drop)**/

proc genmod data $=$ SCHDROP. secschlua07; class Code Region Schoolstatus SchoolLevel; model DropoutNumba/YearlevelSize=StudentTeachRatio Region Schoolstatus SchoolLevel/dist=bin type3; repeated subject $=$ Code $/$ type $=$ exch; run;

/** This is the StudentTeachRatio, Region and SchoolLevel effetcs $\star \star /$

proc genmod data $=$ SCHDROP. secschlua07; class Code Region SchoolLevel;

model DropoutNumba/YearlevelSize=StudentTeachRatio Region SchoolLevel/dist=bin type3; repeated subject $=$ Code $/$ ype $=$ exch; run;

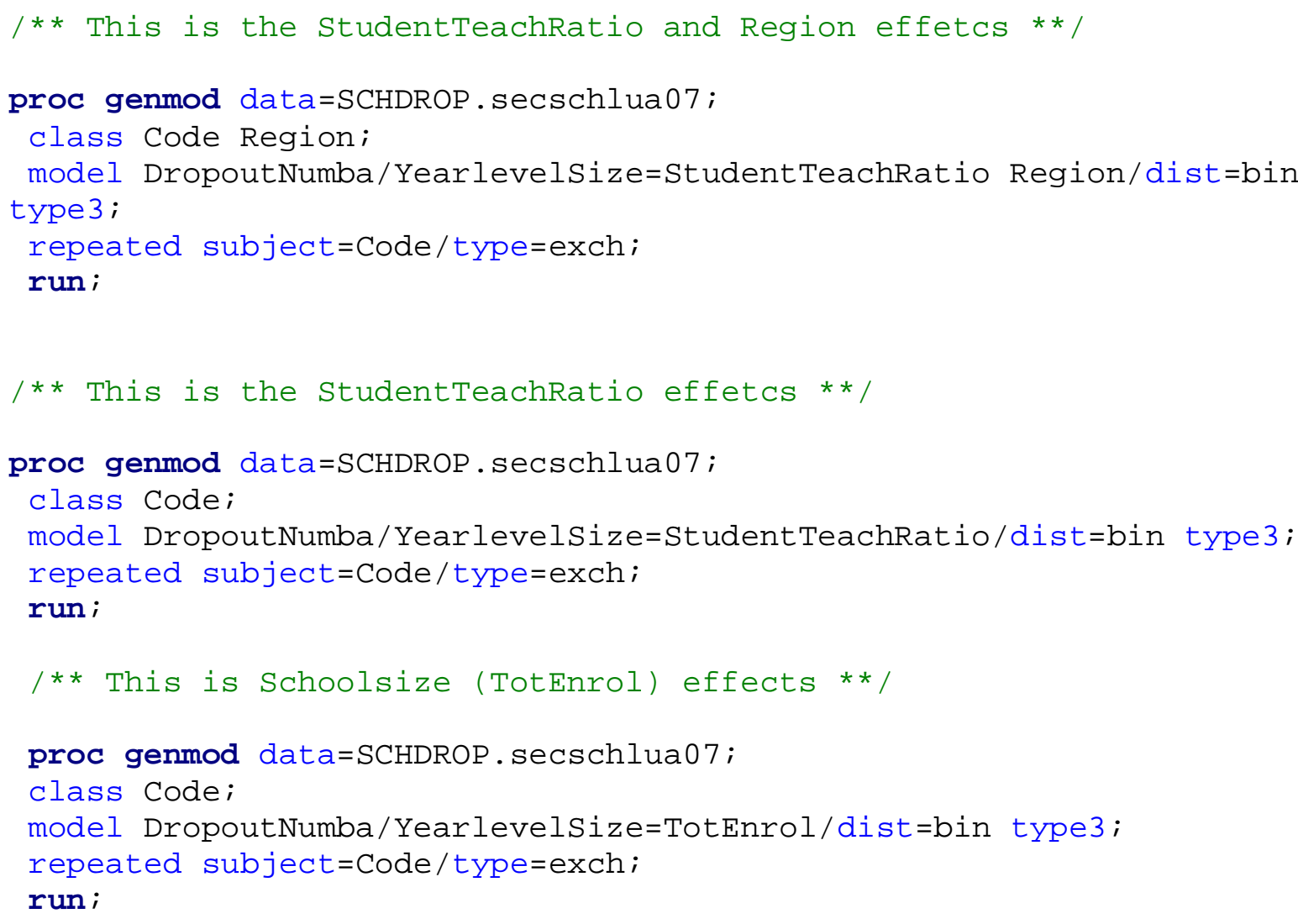




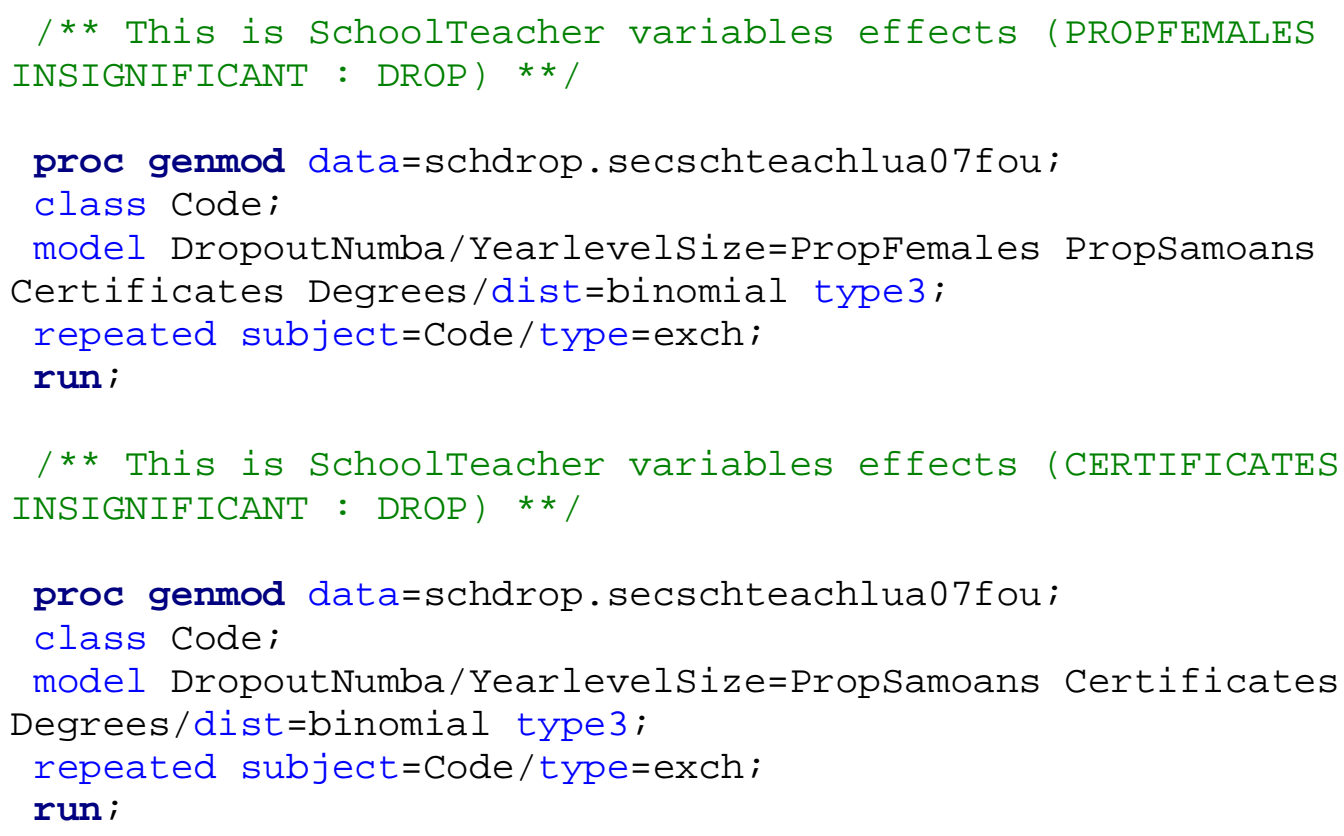


run;

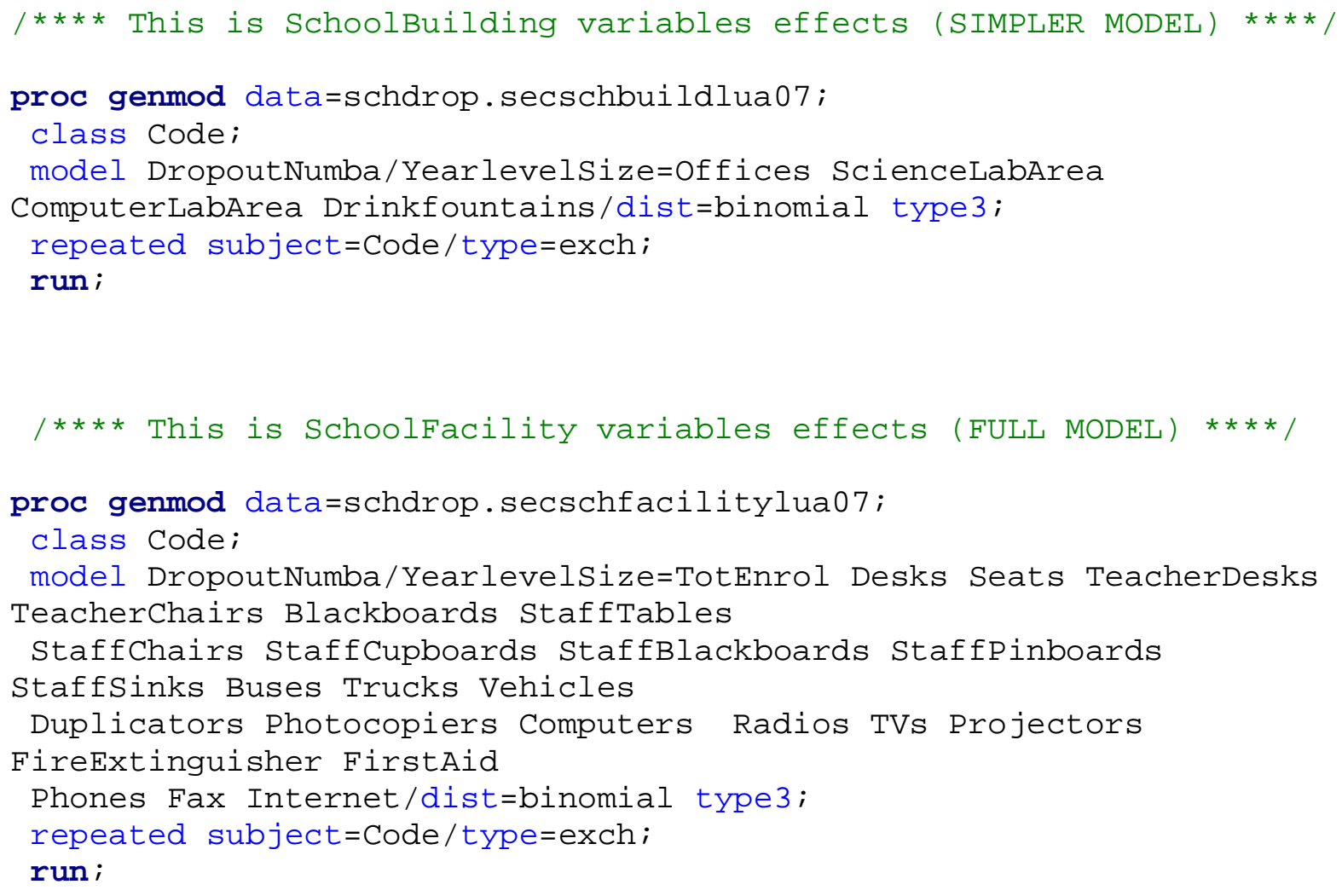

run; 


\section{F. The List of the 21 Educational School Districts in Samoa}

\begin{tabular}{|l|l|}
\hline \multicolumn{1}{|c|}{ SAVAII ISLAND (8) } & \multicolumn{1}{|c|}{ UPOLU ISLAND (13) } \\
\hline 1. Faasaleleaga No.1. & 9. Faleata \\
\hline 2. Faasaleleaga No.2. & 10. Sagaga \\
\hline 3. Itu o Tane No.1. & 11. Aana No.1. \\
\hline 4. Itu o Tane No.2. & 12. Aana No.2. \\
\hline 5. Itu Asau No.1. & 13. Lefaga \\
\hline 6. Itu Asau No.2. & 14. Safata \\
\hline 7. Savaii Sisifo & 15. Falealili \\
\hline 8. Palauli & 16. Lepa ma Lotofaga \\
\hline & 17. Aleipata \\
\hline & 18. Fagaloa \\
\hline & 19. Anoamaa No.1. \\
\hline & 20. Anoamaa No.2. \\
\hline & 21. Vaimauga \\
\hline
\end{tabular}




\section{REFERENCES}

Admassu, K.A. (2011). Primary School Enrolment and Dropout in Ethiopia: Household and School Factors. Availabe at: paa2011.princeton.edu/download. aspx? submissionid=111283.

Agresti, A. (2002). Categorical Data Analysis, $2^{\text {nd }}$ edn. John Wiley \& Sons, Inc, Hoboken, New Jersey.

Allensworth, E. M. (2005). Dropout Rates after High-Stakes Testing in Elementary School: A Study of the Contradictory Effects of Chicago's Efforts to End Social Promotion. Educational Evaluation and Policy Analysis, 27 (4), 341-364.

Antretter, E., et al (2005). Multilevel modelling was a convenient alternative to common regression designs in longitudinal suicide research. Journal of Clinical Epidemiology, 59, 576-586.

Archwamety, T. \& Katsiyannis, A. (1998). Factors Related to Recidivism Among Delinquent Females at a State Correctional Facility. Journal of Child and Family Studies, 7 (1), 59-67.

Barrowman, C., Nutbeam, D. \& Tresidder, J. (2001). Health risks among early school leavers: findings from an Australian study examining the reasons for, and consequences of, early school drop out. Health Education, 101 (Â2), 74-82.

Bozdogan, H. (2000). Akaike's Information Criterion and Recent Developments in Information Complexity. Journal of Mathematical Psychology, 44, 62-91.

Branham, D. (2004). The Wise Man Builds His House Upon the Rock: The Effects of Inadequate School Building Infrastructure on Student Attendance. Social Science Quarterly, 85 (5), 1112-1128.

Cameron, L.A. (2004). Can a public scholarship program successfully reduce school dropouts in a time of economic crisis? Evidence from Indonesia. Available at: www.3ieimpact.org/admin evalutaions/Can a public scholarship program successfully reduce school drop-outs.pdf - Google Chrome. (16/04/2012)

Cameron, L.A. (2002). Did Social Safety Net Scholarships Reduce Drop-Out Rates during the Indonesian Economic Crisis? Policy Research Working Paper 2800, The World Bank, 2002. www-wds.worldbank.org/external/default/WDSContentServer/IW3P/IB/2002/04/05/

Campbell, G. V. (1966). A Review of the Drop out Problem. Peabody Journal of Education, 44 (2), 102-109.

Centre for Innovative School Facilities of Oregon, (Access on: 24/04/12). Quality Facilities Impact Achievement. Available at:

www.cisforegon.org/about/documents/Facility\%20Impacts\%20on\%20Achievement.pdf 
Chapman, C., Laird, J., Ifill, N., \& KewalRamani, A. (2011). Trends in High School Dropout and Completion Rates in the United States: 1972 - 2009 (NCES 2012-006). U.S. Department of Education. Washington, DC.: National Centre for Education Statistics. Retrieved 17/04/2012 from http://nces.ed.gov/pubs2012/2012006.pdf.

Christenson, S. L. \& Thurlow, M. L. (2004). School Dropouts: Prevention Considerations, Interventions, and Challenges. Current Directions in Psychological Science, 13 (1), 36-39.

Cui, J. (2007). QIC program and model selection in GEE analyses. The Strata Journal, 7 (2), 209-220.

ESP (2007). Monitoring School Dropouts: Albania, Kazakhstan, Latvia, Mongolia, Slovakia and Tajikistan. Budapest: Education Support Program (ESP) of the Open Society Institute. www.soros.org/initiatives/esp/articles publications/monitoring 20070607/monitoring 20070 607.pdf

Fijilive (2009). Exams scrapped to lessen school dropouts. Available at: www.fijilive.com/news/2009/07/02/17815.Fijilive

Fischer, M. J., (2010). Immigrant educational outcomes in new destinations: An exploration of high school attrition. Social Science Research, 39, 627-641.

GNA (Ghana News Agency), (2012). Inadequate facilities, cause of school drop-out-rateEducationist. Access on: 24/04/12. Available at:

www.modernghana.com/news/81052/1/inadequate-facilities-cause-of-school-drop-outrat.html.

Goldstein, H. (1995). Multilevel statistical models, $2^{\text {nd }}$ edn. Edward Arnold, London.

Hanley, J.A., et al (2003). Statistical Analysis of Correlated Data Using Generalised Estimating Equations: An Orientation. American Journal of Epidemiology, 157 (4), 364-375.

Hattam, R. \& Smyth, J. (2003). 'Not Everyone Has a Perfect Life': becoming somebody without school. Pedagogy, Culture \& Society, 11 (3), 379-398.

Hedeker, D. \& Gibbons, R.D. (1994). A Random-Effects Ordinal Regression Model for Multilevel Analysis. Biometrics, 50 (4), 933-944.

Hosmer, D.W. \& Lemeshow, S. (2000). Applied Logistic Regression, $2^{\text {nd }}$ edn. John Wiley \& Sons, Inc, New York.

Ilon, L. \& Moock, P. (1991). School Attributes, Household Characteristics, and Demand for Schooling: A Case Study of Rural Peru. International Review of Education / Internationale Zeitschrift für Erziehungswissenschaft / Revue Internationale de l'Education, 37 (4), 429-451. 
Johnson, J., Strange, M. \& Madden, K. (2010). The Rural Dropout Problem: An Invisible Achievement Gap. Rural School and Community Trust, Arlington, VA 22209.

Lamb, S. (2011). TVET and the poor: Challenges and possibilities. International Journal of training Research, 9 (1-2), 60-71.

Lee, V. E. \& Burkam, D. T. (2003). Dropping out of High School: The Role of School Organization and Structure. American Educational Research Journal, 40 (2), 353-393.

Liang, K.Y. \& Zeger, S.L. (1986). Longitudinal data analysis using generalised linear models. Biometrika, 73, 13-22.

Lofstrom, M. (2007). Why Are Hispanic and African-American Dropout Rates So High? IZA (Institute for the Study of Labour) Discussion Paper No. 3265, 53072 Bonn, Germany.

Lubienski, S. T. \& Lubienski, C. (2006). School Sector and Academic Achievement: A Multilevel Analysis of NAEP Mathematics Data. American Educational Research Journal, 43 (4), 651-698.

Ma, X. (2003). Sense of Belonging to School: Can Schools Make a Difference? The Journal of Educational Research, 96 (6), 340-349.

Malin, M. \& Meidment, D. (2003). Education, Indigenous Survival and Well-Being: Emerging Ideas and Programs. The Australian Journal of Indigenous Education, 32, 85-100.

Masaoud, E. \& Stryhn, H. (2010). A simulation study to assess statistical methods for binary repeated measures data. Preventive Veterinary Medicine, 93, 81-97.

Mason, W.M. (2001). Statistical Analysis: Multilevel Methods. International Encyclopedia of the Social \& Behavioural Sciences, 14988-14994.

McCullagh, P. \& Nelder, J.A. (1989). Generalized Linear Models, $2^{\text {nd }}$ edn. Chapman \& Hall, London.

McNeal, Jr. R. B. (1995). Extracurricular Activities and High School Dropouts. Sociology of Education, 68 (1), 62-80.

Meekers, D. \& Ahmed, G. (1999). Pregnancy-Related School Dropouts in Botswana. Population Studies, 53 (2), 195-209.

Ministry of Education, Ethiopia, (2010). Education Statistics Annual Abstract 2001 E.C. /2008-09 G.C./ Education Management Information System, Ministry of Education, Addis Ababa, Ethiopia. 
Ministry of Eucation, Sports and Culture (2007a). Education For All: Mid-Decade Assessment Report. Ministry of Education, Sports and Culture, Samoa.

Ministry of Education, Sports and Culture (2007b). Education Statistical Digest. Policy, Planning and Research Division, Ministry of Education, Sports and Culture, Samoa.

Ministry of Education, Sports and Culture (2006). MESC Strategic Policies and Plan: July 2006 - June 2015. Ministry of Education, Sports and Culture, Samoa.

Naidu, V., Barr, K. \& Seniloli, K. (2007). Poverty Reduction Policies for Fiji. Fijian Studies: A Journal of Contemporary Fiji, 5 (2), 100-121.

Nekatibeb, T. (2002). Low Participation of Female Students in Primary Education: A Case Study of Dropouts from the Amhara and Oromia Regional States in Ethiopia. UNESCO International Institute for Capacity Building in Africa, Addis Ababa, Ethiopia.

North Carolina Department of Public Instruction, (2000). School Size and its relationship to Achievement and Behaviour. Available at: http://www.ncpublicschools.org/docs/data/reports/size.pdf.

Pan, W. (2001). Akaike's information in generalized estimating equations. Biometrics, 57 (1), 120-125.

Pittman, R. B. (1991). Social Factors, Enrollment in Vocational/Technical Courses, and High School Dropout Rates. The Journal of Educational Research, 84 (5), 288-295.

Pittman, R. B. \& Haughwout, P. (1987). Influence of High School Size on Dropout Rate. Educational Evaluation and Policy Analysis, 9 (4), 337 - 343.

Provasnik, S., et.al, (2007). Status of Education in Rural America (NCES 2007-040). National Center for Education Statistics, Institute of Education Sciences, U.S. Department of Education. Washington, DC.

Ream, R. K. \& Rumberger, R. W. (2008). Student Engagement, Peer Social Capital, and School Dropout among Mexican American and Non-Latino White Students. Sociology of Education, 81 (2), 109-139.

Available at: http://www.jstor.org/stable/20452728.

Rumberger, R. W. (2001). Who Drops Out of School and Why. National Academy Press, Washington, D.C, USA.

Rumberger, R. W. \& Palardy, G. J. (2005). Test Scores, Dropout Rates, and Transfer Rates as Alternative Indicators of High School Performance. American Educational Research Journal, 42 (1), 3-42.

Samoa Bureau of Statistics, (2008). Samoa Population and Housing Census Report 2006. SBS, Samoa. 
SAPPROS Nepal, (2011). Improved Physical Facility Reduced Drop Out Rate in the School. Access: 24/04/12. Available at:

www.sappros.org.np/index.php?option=com content\&view=article \&id=104\&Itemid=160

Singh, M. (2008). Dropouts rise: 15pc of our kids don't survive primary school. Available at: www.fijitimes.com/story.aspx?id=87209.

Slaughter, S.J. \& Delwiche, L.D. (2006). The Little SAS ${ }^{\circledR}$ Book for Enterprise Guide ${ }^{\circledR} 4.1$. Cary, NC: SAS Institute Inc.

So'o, et al (Eds.). (2006). Samoa National Human Development Report: Sustainable livelihoods in a changing Samoa. Centre of Samoan Studies: National University of Samoa.

Stan van Alphen (2009): The educational quality of early school leavers and the crossnational variation of their income disadvantage. Educational Research and Evaluation: An International Journal on Theory and Practice, 15 (6), 543-560.

Stone, C.L. (1956). Family Factors in High School Drop-Outs. The Coordinator, 4 (4), 16-23.

Tanja Traag \& Rolf K. W. van der Velden (2011): Early school-leaving in the Netherlands: the role of family resources, school composition and background characteristics in early school-leaving in lower secondary education. Irish Educational Studies, 30 (1), 45-62.

The Rising Nepal, (Access on 24/04/2012). Lack of toilets in Nepal schools make girls dropout. Available at: www.Southasia.oneworld.net/todaysheadlines/lack-of-toilets-in-nepalschools-make-girls-dropout.

Twisk, J.W.R. (2004). Longitudinal data analysis: A Comparison between generalised estimating equations and random coefficient analysis. European Journal of Epidemiology, 19, 769-776.

Twisk, J.W.R., Smidt, N. \& de Ventre, W. (2005). Applied analysis of recurrent events: a practical overview. Journal of Epidemiology \& Community Health, 59, 706-710.

Tyler, J. H. \& Lofstrom, M. (2009). Finishing High School: Alternative Pathways and Dropout Recovery. The Future of Children, 19 (1), 77-103.

UNESCO, (2006). Guidelines for the Asia and Pacific Educational for All Mid-Decade Assessment: Identifying and Reaching the Unreached. UNICEF East Asia and Pacific Regional Office, Thailand, Bangkok 10200. Available at: www.unescobkk.org/fileadmin/user_upload/efa/EFA_MDA/TechGuide_Draft_15Sept.pdf.

UNICEF, (2008). Education in Albania Country Profile.

Availabe at: www.unicef.org/ceecis/Albania.pdf

Veramu, J. C. (2002). Extending the Reach of Schools. Directions: Journal of Educational Studies: Pacific Curriculum Network, 11 (1), 24-27. 
Vosamana, S. (2012). Money woes cause drop-outs.

Available at: $\underline{w w w . f i j i t i m e s . c o m / s t o r y . a s p x ? i d=196361 ~}$

Wang, Z. \& Louis, T.A. (2004). Marginalized Binary Mixed-Effects Models with CovariateDependent Random Effects and Likelihood Inference. Biometrics, 60 (4), 884-891.

Warren, J. R. \& Jenkins, K. N. (2005). High School Exit Examinations and High School Dropout in Texas and Florida, 1971-2000. Sociology of Education, 78 (2), 122-143.

Wils, A. (2004). Late Entrants Leave School Earlier: Evidence From Mozambique. International Review of Education, Springer. Available at: http://www.jstor.org/stable/4151584.

World Bank, (2005). Gender Roles and Impact. Access on: 24/04/12. Available at: www.wsp.org/wsp/Hygiene-Sanitation-Water-Toolkit/BasicPrinciples/GenderRoles.html.

Yi, H., et al., (2011). Dropping out: Why are students leaving junior high in China's poor rural areas? Int. J. Educ. Dev.(2011) doi:10.1016/j.ijedudev.2011.09.002 